Departamento de Señales, Sistemas y

RADIOCOMUNICACIONES

Escuela Técnica Superior de Ingenieros de

TELECOMUNicACióN

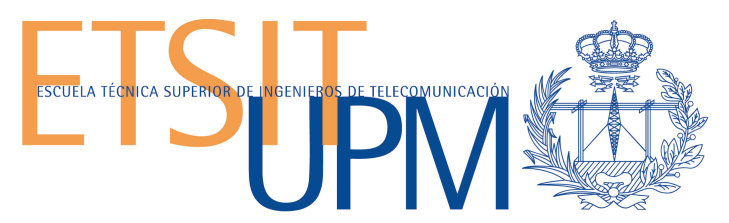

\title{
Applications of Formal Languages to Management of Manned and Unmanned Aircraft
}

Autor:

Director:
Guillermo Frontera Sánchez

Ingeniero en Informática

Juan A. Besada Portas

Doctor Ingeniero de Telecomunicación 

DEPARTMENT:

Departamento de Señales, Sistemas y Radiocomunicaciones

Escuela Técnica Superior de Ingenieros de Telecomunicación

Universidad Politécnica de Madrid

PH.D. THESIS:

Applications of Formal Languages to Management of Manned and Unmanned Aircraft

Author:

Guillermo Frontera Sánchez

Advisor: Juan A. Besada Portas

Committee named by the Rector of Universidad Politécnica de Madrid, on the of 201_.

Committee:

President:

José Ramón Casar Corredera

Universidad Politécnica de Madrid

Member:

Daniel Delahaye

Ecole Nationale de l'Aviation Civile

Member:

Jesús García Herrero

Universidad Carlos III de Madrid

Member:

Miguel A. Vilaplana Ruiz

Boeing Research \& Technology Europe

Secretary:

Gonzalo de Miguel Vela

Universidad Politécnica de Madrid

Reserve:

José Miguel Canino Rodríguez

Universidad de Las Palmas de Gran Canaria

Reserve:

Javier López Leonés

Boeing Research \& Technology Europe

After the defence of the Ph.D. Thesis on the of 201 at the Escuela Técnica Superior de Ingenieros de Telecomunicación, the committee agrees to grant the following qualification:

President

Secretary

Members 



\section{'Fly, you fools!'}

- Gandalf the Grey

in The Lord of the Rings:

The Fellowship of the Ring (1954)

by J.R.R. Tolkien (1892-1973). 



\section{Acknowledgements}

Writing a thesis isn't a short journey. Nor an easy one. For me, it has been a winding road full of ups and downs, where I have enjoyed as much from my achievements as I have suffered from my frustrations. Luckily, I didn't have to make this journey alone.

First of all, I want to thank Prof. Juan Besada for being involved in my dayto-day work. He always offered valuable advice and guidance when I felt stuck in a problem. I also want to thank him and all the professors in our research group - José Ramón, Gonzalo, Ana, and Javier - for everything they have taught me and for offering help whenever I needed it. I am also very grateful to Prof. Dawei $\mathrm{Gu}$, from the University of Leicester, for all his valuable advice.

Much of my research work is the result of a collaboration with Boeing Research \& Technology Europe (BR\&TE). I am grateful to all the people from BR\&TE I have worked with, and very specially to Javier López, who has witnessed this journey from the beginning and from whom I have learned a lot.

In these years, I have met many great people at the Universidad Politécnica de Madrid. Edu, Luca, David — the two of them-, Íñigo, Josué, Rafa, Henar, Iván, Wang, Julie, Ángel - the three of them-, Jaime, Paula, Dani, Laura, Jesús - the two of them-, Miriam... and many more (including all the interns who 'crowded' the research group at some point). Thank you for all the great times we've had together, both in and outside of the university. I wish you the best of luck.

My friends have had to put up with me all these years, and they have helped me keep my sanity —or what's left of it, anyway. Making this journey without the eight people I consider my closest friends wouldn't have been the same. Álex, Andrés, David, Javi, Manu, Óscar, Quique, and Rubén, sois grandes.

And finally, and most importantly, I am very grateful to my family for all their love and support during all these years. In particular, I want to thank my parents, Bártel and Elsa, to whom I owe everything I am and everything I have. 



\section{Abstract}

Future air traffic management (ATM) will require a paradigm shift from today's mainly tactical ATM to trajectory-based operations (TBOs). An increase in the level of automation will also relieve humans - air traffic control officers (ATCOs), flight crew, etc. - from many of the tasks they perform today. Humans will still be central in this future ATM, as decision-makers and managers. These two improvements (TBOs and increased automation) are expected to provide the increase in ATM performance that will allow coping with the expected increase in air transport demand.

Under TBOs, trajectories are negotiated between the airspace user (an airline, pilot, or operator) and the air navigation service provider (ANSP) using a collaborative decision making (CDM) process. A suitable method for sharing aircraft trajectories is necessary for this negotiation. Sharing a whole trajectory would require a high amount of bandwidth, and the shared trajectory might become invalid if the weather forecast changed. Instead, a description of the trajectory, decoupled from the weather conditions, could be shared, so that the actual trajectory could be computed from this trajectory description. This trajectory description should be easy to process using a computing program - as some of the CDM processes will be automated - but also easy to understand for a human operator - who will be supervising the process and making decisions.

This thesis presents a series of formal languages that can be used for this purpose. These languages provide the means to describe aircraft trajectories during all phases of flight, from push back to arrival at the gate. They can also describe trajectories of both manned and unmanned aircraft, including fixedwing and some rotary-wing aircraft (quadrotors).

Some of these languages are tightly interrelated and organized in a language hierarchy. One of the key languages in this hierarchy, the aircraft intent description language (AIDL), had already been developed prior to this thesis. This language was derived from the equations of motion of fixed-wing aircraft, and can provide an unambiguous description of fixed-wing aircraft trajectories. A variant of this language, the quadrotor AIDL (QR-AIDL), is developed in this thesis to allow describing a quadrotor aircraft trajectory with the same level of detail. Then, the intent composite description language (ICDL) is built on top of these two languages, providing more flexibility to describe some parts of the trajectory while leaving others unspecified. The ICDL is used to provide generic descriptions of common aircraft manoeuvres, which can be particularized and combined to form complex descriptions of flight. Another language is built on top of the ICDL, the flight intent description language (FIDL). The 
FIDL specifies high-level requirements on trajectories -including constraints and objectives - , but can use features of the ICDL to provide arbitrary levels of detail in different parts of the flight. The ICDL and FIDL have been developed in collaboration with Boeing Research 85 Technology Europe (BR\&TE). Also, the mission intent description language (MIDL) has been developed to allow describing missions involving multiple aircraft. This language is based on the FIDL and keeps all its expressive power, while it also provides new semantics for describing mission tasks, mission objectives, and constraints involving several aircraft.

In ATM, the movement of aircraft while on the airport surface also has to be monitored and managed. Another formal language has been designed for this purpose, denoted surface movement description language (SMDL). This language does not belong to the language hierarchy described above, and it is based on the clearances used in airport surface operations. Means to express uncertainty and mutability of different parts of the trajectory are also provided.

Finally, the applications of these languages to trajectory prediction and mission planning are explored in this thesis. The concept of trajectory language processing engine (TLPE) is used in these two applications. A TLPE is an ATM function whose main input and output are expressed in any of the languages in the hierarchy described in this thesis. A modular trajectory predictor is defined as a combination of multiple TLPEs, each of them performing a small subtask. Special attention is given to the TLPE that builds the horizontal, vertical, and configuration profiles of the trajectory. In particular, a novel method for the generation of the vertical profile is presented.

The process of planning a mission can also be seen as a TLPE, where the main input is expressed in the MIDL and the output consists of a number of trajectory descriptions - one for each aircraft available in the mission - expressed in the FIDL. A mixed integer linear programming (MILP) formulation for the problem of assigning mission tasks to the available aircraft is provided. In addition, since finding optimal paths between locations is a key problem to mission planning, a novel path finding algorithm is presented. This algorithm can compute near-shortest paths avoiding all obstacles in an urban environment in very short times.

The several formal languages described in this thesis can serve as a standard specification to share trajectory information among different actors in ATM. In combination, these languages can describe trajectories with the necessary level of detail for any application, and can be used to increase automation by exploiting this information using decision support tools (DSTs). Their applications to some basic functions of DSTs, such as trajectory prediction, have been analized. 


\section{Resumen}

En el futuro, la gestión del tráfico aéreo (ATM, del inglés air traffic management) requerirá un cambio de paradigma, de la gestión principalmente táctica de hoy, a las denominadas operaciones basadas en trayectoria. Un incremento en el nivel de automatización liberará al personal de ATM — controladores, tripulación, etc.- de muchas de las tareas que realizan hoy. Las personas seguirán siendo el elemento central en la gestión del tráfico aéreo del futuro, pero lo serán mediante la gestión y toma de decisiones. Se espera que estas dos mejoras traigan un incremento en la eficiencia de la gestión del tráfico aéreo que permita hacer frente al incremento previsto en la demanda de transporte aéreo.

Para aplicar el concepto de operaciones basadas en trayectoria, el usuario del espacio aéreo (la aerolínea, piloto, u operador) y el proveedor del servicio de navegación aérea deben negociar las trayectorias mediante un proceso de toma de decisiones colaborativo. En esta negociación, es necesaria una forma adecuada de compartir dichas trayectorias. Compartir la trayectoria completa requeriría un gran ancho de banda, y la trayectoria compartida podría invalidarse si cambiase la predicción meteorológica. En su lugar, podría compartirse una descripción de la trayectoria independiente de las condiciones meteorológicas, de manera que la trayectoria real se pudiese calcular a partir de dicha descripción. Esta descripción de la trayectoria debería ser fácil de procesar usando un programa de ordenador - ya que parte del proceso de toma de decisiones estará automatizado-, pero también fácil de entender para un operador humano -que será el que supervise el proceso y tome las decisiones oportunas-.

Esta tesis presenta una serie de lenguajes formales que pueden usarse para este propósito. Estos lenguajes proporcionan los medios para describir trayectorias de aviones durante todas las fases de vuelo, desde la maniobra de push-back (remolcado hasta la calle de rodaje), hasta la llegada a la terminal del aeropuerto de destino. También permiten describir trayectorias tanto de aeronaves tripuladas como no tripuladas, incluyendo aviones de ala fija y cuadricópteros.

Algunos de estos lenguajes están estrechamente relacionados entre sí, y organizados en una jerarquía. Uno de los lenguajes fundamentales de esta jerarquía, llamado aircraft intent description language (AIDL), ya había sido desarrollado con anterioridad a esta tesis. Este lenguaje fue derivado de las ecuaciones del movimiento de los aviones de ala fija, y puede utilizarse para describir sin ambigüedad trayectorias de este tipo de aeronaves. Una variante de este lenguaje, denominada quadrotor AIDL (QR-AIDL), ha sido desarrollada en esta tesis para permitir describir trayectorias de cuadricópteros con el mismo nivel de detalle. Seguidamente, otro lenguaje, denominado intent composite description langua- 
ge (ICDL), se apoya en los dos lenguajes anteriores, ofreciendo más flexibilidad para describir algunas partes de la trayectoria y dejar otras sin especificar. El ICDL se usa para proporcionar descripciones genéricas de maniobras comunes, que después se particularizan y combinan para formar descripciones complejas de un vuelo. Otro lenguaje puede construirse a partir del ICDL, denominado flight intent description language (FIDL). El FIDL especifica requisitos de alto nivel sobre las trayectorias - incluyendo restricciones y objetivos-, pero puede utilizar características del ICDL para proporcionar niveles de detalle arbitrarios en las distintas partes de un vuelo. Tanto el ICDL como el FIDL han sido desarrollados en colaboración con Boeing Research \& Technology Europe (BR\&TE). También se ha desarrollado un lenguaje para definir misiones en las que interactúan varias aeronaves, el mission intent description language (MIDL). Este lenguaje se basa en el FIDL y mantiene todo su poder expresivo, a la vez que proporciona nuevas semánticas para describir tareas, restricciones y objetivos relacionados con la misión.

En ATM, los movimientos de un avión en la superficie de aeropuerto también tienen que ser monitorizados y gestionados. Otro lenguaje formal ha sido diseñado con este propósito, llamado surface movement description language (SMDL). Este lenguaje no pertenece a la jerarquía de lenguajes descrita en el párrafo anterior, y se basa en las clearances (autorizaciones del controlador) utilizadas durante las operaciones en superficie de aeropuerto. También proporciona medios para expresar incertidumbre y posibilidad de cambios en las distintas partes de la trayectoria.

Finalmente, esta tesis explora las aplicaciones de estos lenguajes a la predicción de trayectorias y a la planificación de misiones. El concepto de trajectory language processing engine (TLPE) se usa en ambas aplicaciones. Un TLPE es una función de ATM cuya principal entrada y salida se expresan en cualquiera de los lenguajes incluidos en la jerarquía descrita en esta tesis. El proceso de predicción de trayectorias puede definirse como una combinación de TLPEs, cada uno de los cuales realiza una pequeña sub-tarea. Se le ha dado especial importancia a uno de estos TLPEs, que se encarga de generar el perfil horizontal, vertical y de configuración de la trayectoria. En particular, esta tesis presenta un método novedoso para la generación del perfil vertical.

El proceso de planificar una misión también se puede ver como un TLPE donde la entrada se expresa en MIDL y la salida consiste en cierto número de trayectorias - una por cada aeronave disponible - descritas utilizando FIDL. Se ha formulado este problema utilizando programación entera mixta. Además, dado que encontrar caminos óptimos entre distintos puntos es un problema fundamental en la planificación de misiones, también se propone un algoritmo de búsqueda de caminos. Este algoritmo permite calcular rápidamente caminos cuasi-óptimos que esquivan todos los obstáculos en un entorno urbano.

Los diferentes lenguajes formales definidos en esta tesis pueden utilizarse como una especificación estándar para la difusión de información entre distintos actores de la gestión del tráfico aéreo. En conjunto, estos lenguajes permiten describir trayectorias con el nivel de detalle necesario en cada aplicación, y se pueden utilizar para aumentar el nivel de automatización explotando esta información utilizando sistemas de soporte a la toma de decisiones. La aplicación de estos lenguajes a algunas funciones básicas de estos sistemas, como la predicción de trayectorias, han sido analizadas. 


\section{Contents}

Acknowledgements vii

$\begin{array}{ll}\text { Abstract } & \text { ix }\end{array}$

Resumen $\quad$ xi

Contents xiii

Acronyms $\quad$ xix

$\begin{array}{ll}\text { List of Tables } & \text { xxiii }\end{array}$

List of Figures $\quad$ Xxv

1 Introduction $\quad 1$

1.1 Future challenges in ATM . . . . . . . . . . . . . 2

1.1.1 Trajectory-based operations ............. 3

1.1.2 Unmanned aircraft . . . . . . . . . . . . . . . 3

1.2 Formal languages . . . . . . . . . . . . . . . . 4

1.3 Problem statement . . . . . . . . . . . . . . . 4

1.4 Summary of this work ................. 5

$\begin{array}{lll}\text { I } & \text { Background and Literature Survey } & 7\end{array}$

2 Specification of flight-related data 9

2.1 Specification of trajectories . . . . . . . . . . . . 10

2.2 Specification of aircraft intent . . . . . . . . . . . . . . 11

2.2.1 Aircraft intent description language . . . . . . . . . . 12

2.2.2 Other specifications of aircraft intent . . . . . . . . 12

2.3 Specification of flight intent . . . . . . . . . . . . . . 14

2.3.1 ICAO Flight Plan . . . . . . . . . . . . . . 15

2.3.2 Other specifications of flight . . . . . . . . 15 
2.3.3 Additional flight information . . . . . . . . . . . 16

2.4 Specification of mission intent . . . . . . . . . . . . . 17

2.5 Specification of airport surface movement . . . . . . . . . . . 19

2.6 Final words . . . . . . . . . . . . . . . . . . . 20

3 Prediction of aircraft trajectories $\quad 21$

3.1 Structure of a trajectory predictor . . . . . . . . . . . . . 22

3.2 Models of aircraft performance . . . . . . . . . . . . . 24

3.3 Development of trajectory predictors . . . . . . . . . . 25

3.3.1 CTAS trajectory synthesizer . . . . . . . . . 25

3.3.2 PHARE trajectory predictor . . . . . . . . . 26

3.3.3 Trajectory prediction in a FMS . . . . . . . . . . 28

3.3.4 Other approaches to trajectory prediction . . . . . . 28

3.4 Final words . . . . . . . . . . . . . . . . . . . 29

4 Management of a mission involving a fleet of UAs 31

4.1 Mission management system architecture . . . . . . . . . . . . 32

4.2 Optimal distribution of tasks among UAs . . . . . . . . . 33

4.3 Finding paths among obstacles . . . . . . . . . . . 35

4.3.1 The two-dimensional case . . . . . . . . . . . 35

4.3.2 The three-dimensional case . . . . . . . . . . 36

4.4 Final words . . . . . . . . . . . . . . . . . 36

II A Hierarchy of Formal Languages 37

$5 \quad$ Language hierarchy overview $\quad 39$

6 Aircraft intent description language $\quad 43$

6.1 Derivation of the AIDL . . . . . . . . . . . . . . 44

6.2 Aircraft intent for quadrotors . . . . . . . . . . . . . . 47

6.2.1 Model of six mechanical DoF . . . . . . . . . . . 47

6.2.2 Model of three mechanical DoF . . . . . . . . . . . 53

6.3 Language description . . . . . . . . . . . . . . . . . . . 59

6.3 .1 Instructions . . . . . . . . . . . . . 59

6.3 .2 Intent . . . . . . . . . . . . . . 65

6.4 Graphical representation . . . . . . . . . . . . . . 66

6.5 Examples ...................... 67

6.6 Final words ...................... 67 
7 Intent composite description language $\quad 69$

7.1 Language description . . . . . . . . . . . . . . . . . 70

7.1.1 Elementary composites . . . . . . . . . . 70

7.1 .2 Triggers ................... 72

7.1 .3 Templates .................... 73

7.1.4 Composite operations ............. . . 75

7.2 Graphical representation . . . . . . . . . . . . . . 80

7.3 Examples ....................... 81

7.3.1 Commercial flight . . . . . . . . . . . 81

7.3.2 Quadrotor flight .............. 81

7.4 Final words . . . . . . . . . . . . . . . 84

8 Flight intent description language $\quad \mathbf{8 7}$

8.1 Language description . . . . . . . . . . . . . . . . . . 88

8.1.1 Flight segments . . . . . . . . . . . . 88

8.1.2 Aggregations of flight segments . . . . . . . . . 89

8.1 .3 Constraints .................. 89

8.1 .4 Objectives ........................ 91

8.2 Graphical representation . . . . . . . . . . . . . . 92

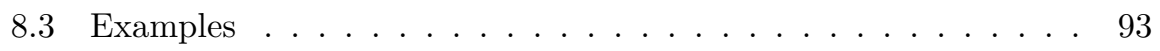

8.3.1 Commercial flight . . . . . . . . . . . . 93

8.3 .2 Firefight flight . . . . . . . . . . . . . . 94

8.3.3 UA reconnaissance mission . . . . . . . . . . . 98

8.4 Final words . . . . . . . . . . . . . . . . . 99

9 Mission intent description language 101

9.1 Language description . . . . . . . . . . . . . . . . . . . 102

9.1.1 Tasks ....................... 102

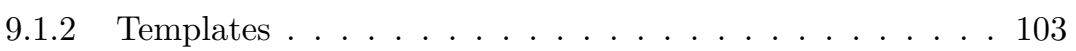

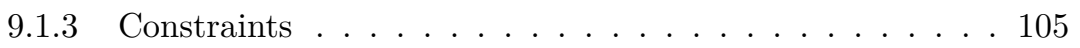

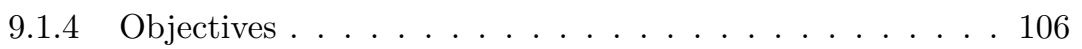

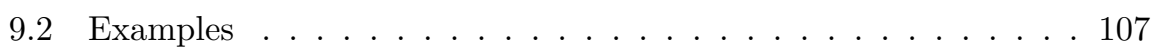

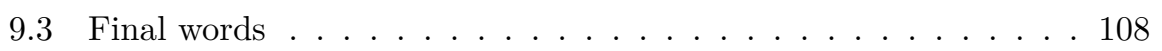

10 Surface movement description language $\quad 109$

10.1 Language description . . . . . . . . . . . . . . . . . . . 110

10.1 .1 Instructions . . . . . . . . . . . . . . . . 110

10.1 .2 Routes..................... 113

10.2 Graphical representation . . . . . . . . . . . . . . . . 114

10.3 Examples . . . . . . . . . . . . . . . . . . 114

10.4 Final words .......................... 118 
11 Trajectory prediction using formal languages $\quad 123$

11.1 Trajectory language processing engines . . . . . . . . . . . . . 123

11.1.1 Enrichment engines . . . . . . . . . . . . . . . 124

11.1.2 Translation engines . . . . . . . . . . . . . 125

11.1.3 Transcription engines . . . . . . . . . . . . 125

11.1.4 Edition engines . . . . . . . . . . . . . . 126

11.2 Engine descriptions . . . . . . . . . . . . . . . . 126

11.2.1 Trajectory computation engine . . . . . . . . . . 127

11.2.2 User preferences engine . . . . . . . . . . . . . . 128

11.2.3 Operational context engine . . . . . . . . . . . 129

11.2.4 Aircraft performance limitations engine . . . . . . . . 130

11.2.5 Intent generation core process engine . . . . . . . . . 130

11.2.6 Intent generation engine . . . . . . . . . . . . . . 131

11.2.7 Trajectory prediction engine . . . . . . . . . . . 132

11.3 Examples . . . . . . . . . . . . . . . . 133

11.4 Final words . . . . . . . . . . . . . . . . . . 140

12 The core of the intent generation process 143

12.1 Process description . . . . . . . . . . . . . . . . 143

12.2 Horizontal Profile Generation . . . . . . . . . . . . . . . . 145

12.3 Vertical Profile Generation . . . . . . . . . . . . . . . . . . 147

12.3.1 Most promising criterion . . . . . . . . . . . 151

12.3.2 Micro-strategies . . . . . . . . . . . . . . 153

12.4 Results . . . . . . . . . . . . . . . . . 154

12.5 Final words . . . . . . . . . . . . . . . . 161

13 Computation of quadrotor trajectories 163

13.1 Engine description . . . . . . . . . . . . . . . . . 164

13.2 Operation integration . . . . . . . . . . . . 165

13.2.1 Combination $1 \ldots \ldots \ldots 165$

13.2.2 Combination $2 \ldots \ldots$. . . . . . . . . . 167

13.2.3 Combination 3 . . . . . . . . . . . . . . 169

13.2.4 Combination $4 \ldots \ldots \ldots 171$

13.2 .5 Combination $5 \ldots \ldots \ldots 173$

13.2.6 Combination $6 \ldots \ldots \ldots 175$

13.3 Example . . . . . . . . . . . . . . . . 177

13.4 Final words . . . . . . . . . . . . . . . 180 
14 Mission management using formal languages

14.1 Translating mission intent into flight intent . . . . . . . . . . 183

14.1.1 Problem formulation . . . . . . . . . . . . . 183

14.1.2 Partial formulation . . . . . . . . . . . . 185

14.1.3 Alternative partial formulation . . . . . . . . . . 187

14.1.4 Complete formulation . . . . . . . . . . . 189

14.1.5 Example.................... 196

14.2 Computing paths in an urban environment . . . . . . . . 199

14.2.1 Approximate Shortest Paths . . . . . . . . . . . 200

14.2 .2 Visibility Graph . . . . . . . . . . . . . . . . . 201

14.2.3 Filtering obstacles . . . . . . . . . . . . . 204

14.2.4 Building a path incrementally . . . . . . . . 205

14.2 .5 Results ................... . 207

14.3 Final words . . . . . . . . . . . . . . . . . . . . . 212

IV Conclusions and Bibliography 215

$\begin{array}{lr}15 \text { Conclusions } & 217\end{array}$

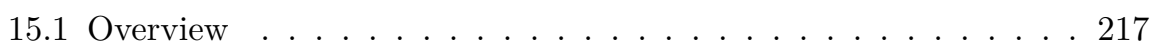

15.2 Contributions . . . . . . . . . . . . . . . . 222

15.3 Further work . . . . . . . . . . . . . . . . 223

$\begin{array}{ll}\text { Bibliography } & 225\end{array}$

V Appendices 239

A AIDL for commercial fixed-wing aircraft $\quad 241$

A.1 Language description . . . . . . . . . . . . . . . . . . . . . 241

A.1.1 Instructions . . . . . . . . . . . . . . . . . 242

A.1.2 Intent .................... 244

B Dynamic model of a quadrotor $\quad 247$

B.1 Physical effects . . . . . . . . . . . . . . . . . 249

B.2 Equations of motion . . . . . . . . . . . . . . . . 251

B.3 Quadrotor parameters ... . . . . . . . . . . . 252 
C Basic ICDL templates $\quad 255$

C.1 Commercial flight templates . . . . . . . . . . . . . 255

C.1.1 Vertical profile . . . . . . . . . . . . . . . 255

C.1.2 Configuration profile . . . . . . . . . . . . . . 259

C.1.3 Lateral profile. . . . . . . . . . . . . . . . . . . 260

C.2 Quadrotor templates . . . . . . . . . . . . . . . 261

C.2.1 Lateral profile. . . . . . . . . . . . . . . . . . . . . . . . . . . . . . . . . . . . . .

C.2.2 Vertical profile . . . . . . . . . . . . . . . 262

C.2.3 Lateral and vertical profiles . . . . . . . . . . . . 263

C.2.4 Orientation profile . . . . . . . . . . . . . . . 264

D Formal specification of intent-based languages $\quad 267$

D.1 Quadrotor AIDL . . . . . . . . . . . . . . . . 268

D.2 Intent composite description language . . . . . . . . . . 270

D.3 Flight intent description language . . . . . . . . . . . . . 272

D.4 Mission intent description language . . . . . . . . . . . 273 


\section{Acronyms}

$\begin{array}{ll}\text { 2D } & \text { two-dimensional } \\ \text { 2ESP } & \text { 2D Euclidean shortest path } \\ \text { 3D } & \text { three-dimensional } \\ \text { 3ESP } & \text { 3D Euclidean shortest path } \\ \text { 4D } & \text { four-dimensional } \\ \text { 4DTRAD } & \text { 4D trajectory data link } \\ & \\ \text { A-SMGCS } & \text { advanced surface movement guidance and con- } \\ & \text { trol system } \\ \text { AFV } & \text { autonomous flying vehicle } \\ \text { AGLAS } & \text { airfield ground lighting automation system } \\ \text { AIDL } & \text { aircraft intent description language } \\ \text { AIGE } & \text { aircraft intent generation engine } \\ \text { AIGI } & \text { aircraft intent generation infrastructure } \\ \text { AIP } & \text { aeronautical information publication } \\ \text { AIXM } & \text { aeronautical information exchange model } \\ \text { AMM } & \text { aircraft motion model } \\ \text { AMPL } & \text { A Mathematical Programming Language } \\ \text { ANSP } & \text { air navigation service provider } \\ \text { ANU } & \text { Australian National University } \\ \text { APLE } & \text { aircraft performance limitations engine } \\ \text { APM } & \text { aircraft performance model } \\ \text { ARINC } & \text { Aeronautical Radio, Inc. } \\ \text { ATC } & \text { air traffic control } \\ \text { ATCO } & \text { air traffic control officer } \\ \text { ATM } & \text { air traffic management } \\ \text { ATS } & \text { air traffic service } \\ & \\ \text { BADA } & \text { base of aircraft data } \\ \text { BLOVL } & \text { base line-oriented visibility line } \\ \text { BR\&TE } & \text { Boeing Research \& Technology Europe } \\ & \\ \text { C-BML } & \text { coalition battle management language } \\ \text { C2IS } & \text { command and control information system } \\ \text { CAASD } & \text { Center for Advanced Aviation System Develop- } \\ & \text { ment } \\ \text { CAS } & \text { calibrated airspeed } \\ & \end{array}$




\begin{tabular}{|c|c|}
\hline CDA & continuous descent approach \\
\hline CDM & collaborative decision making \\
\hline CENA & Centre d'Études de la Navigation Aérienne \\
\hline $\mathrm{CI}$ & cost index \\
\hline CITRAC & common interface for trajectory computation \\
\hline $\mathrm{COE}$ & composite optimization engine \\
\hline COTS & commercial off-the-shelf \\
\hline CPDLC & controller-pilot data link communications \\
\hline $\mathrm{CPU}$ & central processing unit \\
\hline CTAS & Center-TRACON Automation System \\
\hline CTE & composite translation engine \\
\hline DAE & differential algebraic equation \\
\hline DME & distance measuring equipment \\
\hline DoA & domain of application \\
\hline DoF & degree of freedom \\
\hline DST & decision support tool \\
\hline EFMS & experimental flight management system \\
\hline EM & Earth model \\
\hline ESF & energy share factor \\
\hline ETSIT & $\begin{array}{l}\text { Escuela Técnica Superior de Ingenieros de Tele- } \\
\text { comunicación }\end{array}$ \\
\hline EUROCAE & $\begin{array}{l}\text { European Organisation for Civil Aviation Equip- } \\
\text { ment }\end{array}$ \\
\hline EUROPA & $\begin{array}{l}\text { extensible universal remote operations planning } \\
\text { architecture }\end{array}$ \\
\hline FAA & Federal Aviation Administration \\
\hline FAP & final approach point \\
\hline FCS & flight control system \\
\hline FIDL & flight intent description language \\
\hline FMS & flight management system \\
\hline FOIPS & flight object interoperability proposed standard \\
\hline FSM & finite state machine \\
\hline FW-AIDL & fixed-wing AIDL \\
\hline GNSS & global navigation satellite system \\
\hline GPS & global positioning system \\
\hline IAF & initial approach fix \\
\hline IAS & indicated airspeed \\
\hline $\mathrm{IC}$ & initial condition \\
\hline $\mathrm{ICAO}$ & International Civil Aviation Organization \\
\hline ICDL & intent composite description language \\
\hline $\mathrm{IF}$ & intermediate fix \\
\hline IFR & instrument flight rules \\
\hline IGCPE & intent generation core process engine \\
\hline IGE & intent generation engine \\
\hline
\end{tabular}


IVP initial value problem

MEI mission engine infrastructure

MIDL mission intent description language

MILP mixed integer linear programming

MIPE mission intent processing engine

MPE mission planning engine

NASA National Aeronautics and Space Administration

NextGen Next Generation Air Transportation System

OCE operational context engine

OCM operational context model

ODE ordinary differential equation

OS4 omnidirectional stationary flying outstretched robot

PDL procedure description language

PHARE Programme for Harmonised ATM Research in Eurocontrol

PIDL payload intent description language

PMI performance measurement infrastructure

PPE path planning engine

QR-AIDL quadrotor AIDL

RAM random-access memory

RNAV area navigation

RNP required navigation performance

RTA required time of arrival

RTCA Radio Technical Commission for Aeronautics

SDR sequential DAEs resolution

SESAR Single European Sky ATM Research

SID standard instrument departure route

SMDL surface movement description language

SRP swarm routing problem

STAR standard arrival route

TAL temporal action logic

TAS true airspeed

TBO trajectory-based operation

TCE trajectory computation engine

TCI trajectory computation infrastructure

TE trajectory engine

TFPOP threaded forward-chaining partial order planner

TLPE trajectory language processing engine

TMA terminal manoeuvring area

TOD top of descent

TPE trajectory prediction engine 
TRACON terminal radar approach control

travelling salesman problem

task specification tree

UA unmanned aircraft

UAS unmanned aircraft system

UAV unmanned aerial vehicle

UPE user preferences engine

UPM user preferences model

VFR visual flight rules

VHF very high frequency

VOR VHF omnidirectional range

VRP vehicle routing problem

VRPTW vehicle routing problem with time windows

VTOL vertical take-off and landing

WM weather model 


\section{List of Tables}

2.1 Simple GenProf expression . . . . . . . . . . . . . . 13

5.1 Summary of language properties $\ldots \ldots \ldots \ldots \ldots$

6.1 Description of variables in quadrotor AMM. . . . . . . . . . . 48

6.2 Generic and representative constraints for solvability analysis. . . 50

6.3 New representative constraints for solvability analysis. . . . . . 55

6.4 Results of Pryce's structural analysis for several combinations of

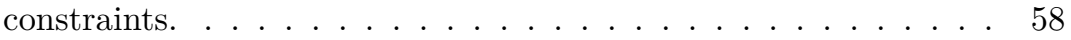

6.5 AIDL instructions for quadrotor aircraft. . . . . . . . . . 60

6.6 Instruction profiles and related threads. . . . . . . . . . . . 65

6.7 Effect groups and assigned colours. . . . . . . . . . . . . 66

8.1 Firefighting flight point coordinates . . . . . . . . . . 96

10.1 Summary of initial instructions . . . . . . . . . . . . . 112

10.2 Summary of in-route instructions . . . . . . . . . . . . 113

10.3 Summary of final instructions . . . . . . . . . . . . . . 113

12.1 Trajectory computation times and costs $\ldots \ldots \ldots \ldots$

12.2 Optimization result examples . . . . . . . . . . . . 160

13.1 Waypoints of flight at ETSIT . . . . . . . . . . . 177

14.1 Time windows in example mission. . . . . . . . . . . . 196

14.2 Intervals between visits and periodical visits ending times. . . . 196

14.3 Time costs of travelling from $T_{j_{1}}$ to $T_{j_{2}} \ldots \ldots \ldots \ldots$

14.4 Time costs of travelling from $A_{i}$ 's initial position to $T_{j}$. . . 197

14.5 Route assigned to aircraft $A_{1} \ldots \ldots \ldots \ldots \ldots$

14.6 Route assigned to aircraft $A_{2} \ldots \ldots \ldots \ldots 7$. . . . . 197

14.7 SubS algorithm results . . . . . . . . . . . . . . . . . . . . 209

14.8 APPR. algorithm results . . . . . . . . . . . . . . . . 209

14.9 VLB3D algorithm results . . . . . . . . . . . . . . . . 210 
14.10APVL algorithm results . . . . . . . . . . . . . . . 210

A.1 AIDL instructions for fixed-wing aircraft. . . . . . . . . . . 242

A.2 Instruction profiles and related threads. . . . . . . . . . 245

B.1 Quadrotor parameters . . . . . . . . . . . . . 253 


\section{List of Figures}

$2.12 \mathrm{D}$ representation of ellipsoids describing uncertainty of individual trajectories. . . . . . . . . . . . . . . . . . 11

2.2 Graphical representation of the GenProf expression. . . . . . . . 13

2.3 Examples of path object shapes. . . . . . . . . . . . . . . . 14

2.4 Example Petri net "mission". . . . . . . . . . . . . . . . 18

2.5 Taxi clearances on an enhanced flight deck. . . . . . . . . . . . . 19

2.6 Taxi route guidance on an enhanced flight deck. . . . . . . . . . . 19

3.1 Trajectory predictor structure. . . . . . . . . . . . . 23

3.2 Schematic view of a trajectory predictor. . . . . . . . . . . 24

3.3 Types of turn in CTAS. . . . . . . . . . . . . . . 25

3.4 Example phase table. . . . . . . . . . . . . . . 27

4.1 Hierarchical control architecture. . . . . . . . . . . . . . . . 32

4.2 Global system architecture. . . . . . . . . . . . . . 33

5.1 Relationships between languages in the hierarchy. . . . . . . . . . 41

6.1 Different operations along flight in AIDL. . . . . . . . . . . . 47

6.2 QR-AIDL instruction in graphical notation. . . . . . . . . . . 66

6.3 Simple QR-AIDL intent. . . . . . . . . . . . . . . 67

6.4 QR-AIDL intent. . . . . . . . . . . . 68

7.1 Elementary composite built from the combination of AIDL instructions. . . . . . . . . . . . . . . 72

7.2 'Level flight' template. . . . . . . . . . . . . . . . . . . . 73

7.3 Elementary composite created from the 'level flight' template. . . 74

7.4 Operations on composites. . . . . . . . . . . . . 76

7.5 Valid composite operations . . . . . . . . . . . . . 78

7.6 Invalid composite operations . . . . . . . . . . . . . . 79

7.7 Use of arrows for indicating referenced triggers. . . . . . . . . . . 80

7.8 Example commercial flight in ICDL. . . . . . . . . . . . . . 82 
7.9 Example commercial flight in AIDL. . . . . . . . . . . . . . 83

7.10 Example quadrotor flight in ICDL. . . . . . . . . . . . . 85

8.1 Graphical representation of flight segment aggregations. . . . . . 93

8.2 Minimum FIDL example. . . . . . . . . . . . . . . . 93

8.3 Firefighter FIDL example. . . . . . . . . . . . . . . . . . . . . . . . . . . 95

8.4 Firefighter example trajectory. . . . . . . . . . . . . 96

8.5 Firefighter ICDL example. . . . . . . . . . . . . . . . . 97

8.6 UA FIDL examples. . . . . . . . . . . . . . . . . . 98

10.1 SMDL instructions. . . . . . . . . . . . . . . . . 115

10.2 Simple SMDL route. . . . . . . . . . . . . . . . . . 116

10.3 Completely defined aircraft route on the airport surface map. . . 117

10.4 Partially defined aircraft route on the airport surface map. . . . . 117

10.5 Aircraft routes in Adolfo Suárez Madrid-Barajas airport. . . . . . 118

11.1 Trajectory language processing engine. . . . . . . . . . . . . 124

11.2 Trajectory computation engine. . . . . . . . . . . . . . . . 127

11.3 User preferences engine. . . . . . . . . . . . . . . . . . . 128

11.4 Operational context engine. . . . . . . . . . . . . . . . . 129

11.5 Aircraft performance limitations engine. . . . . . . . . . . 130

11.6 Intent generation core process engine. . . . . . . . . . . . . 131

11.7 Intent generation engine. . . . . . . . . . . . . . . . . 132

11.8 Trajectory prediction engine. . . . . . . . . . . . . . 132

11.9 TERTO6C STAR and relevant constraints. . . . . . . . . . 134

11.10Details of the approach from ENETA to GCLP airport. . . . . 135

11.11Aircraft intent resulting from translation at the IGE. . . . . . 138

11.12Trajectory generated from the resulting aircraft intent. . . . . . 139

11.13Detail of the approach manoeuvre. . . . . . . . . . . . . . . 139

11.14Altitude and speed of the approach to GCLP. . . . . . . . . 140

12.1 Intent generation core process. . . . . . . . . . . . . . . . 144

12.2 Example input and output of the horizontal profile generation. . 146

12.3 Manoeuvres to turn at a point. . . . . . . . . . . . . . 147

12.4 Merging of altitude constraints. . . . . . . . . . . . . 148

12.5 Example context. . . . . . . . . . . . . . . . . . 148

12.6 Vertical profile building flowchart. . . . . . . . . . . . . . . 149

12.7 Context expansion. . . . . . . . . . . . . . . . . . . 151

12.8 Trajectories produced by the IGCPE. . . . . . . . . . 155

12.9 Composite generated by the IGCPE. . . . . . . . . . . . 156

12.10Barometric altitude of flights along distance. . . . . . . . . 157 
12.11TAS of flights along flight distance. . . . . . . . . . . . 157

12.12Altitude and speed of generated flight (with extra constraint). . 158

12.13Composite generated by the IGCPE (with extra constraint). . . 159

13.1 Trajectory computation engine for quadrotor aircraft. . . . . . 163

13.2 Operations in QR-AIDL word. . . . . . . . . . . . . 164

13.3 Operation using the first combination of instructions. . . . . . 165

13.4 Evolution of the aircraft's coordinates with time. . . . . . . 166

13.5 Operation using the second combination of instructions. . . . . 167

13.6 Evolution of several variables of the aircraft's state vector with time (combination two). . . . . . . . . . . . . . . 168

13.7 Operation using the third combination of instructions. . . . . . 169

13.8 Evolution of several variables of the aircraft's state vector with time (combination three) . . . . . . . . . . . . 170

13.9 Operation using the fourth combination of instructions. . . . . 171

13.10Evolution of several variables of the aircraft's state vector with time (combination four). . . . . . . . . . . . . . 172

13.11Operation using the fifth combination of instructions. . . . . . 173

13.12Evolution of several variables of the aircraft's state vector with time (combination five). . . . . . . . . . . . . . 174

13.13Operation using the sixth combination of instructions. . . . . . 175

13.14Evolution of several variables of the aircraft's state vector with time (combination six). . . . . . . . . . . . 176

13.15Aircraft intent to be computed by the TCE. . . . . . . . . 178

13.16Detail of the UA flight. . . . . . . . . . . . . . . 179

13.17Altitude and speed of the flight around the ETSIT. . . . . . . 179

14.1 The mission planning engine and the path planning engine. . . . 182

14.2 Target assignment on a map. . . . . . . . . . . . . . . 198

14.3 Targets visited by aircraft along time. . . . . . . . . . . . . . 198

14.4 Examples of vertical polyhedra. . . . . . . . . . . . . 200

14.5 Two-dimensional visibility graph for three levels. . . . . . . . . 202

14.6 Connecting vertices and their vertical projections in different levels.202

14.7 Edges added by the pair connecting process. . . . . . . . . . 203

14.8 Different paths generated by Core. . . . . . . . . . . . 205

14.9 Example execution of the InCREMENTALPATH algorithm. . . . . 206

14.10Example of different paths generated by each algorithm for the same scenario. . . . . . . . . . . . . . . . . . . 208

14.11Mean and 95th percentile of running time of algorithms versus number of obstacles. . . . . . . . . . . . . . . . . . 211

14.12Mean length ratio of algorithms' paths versus number of obstacles. 212 
15.1 Representation of abstraction capabilities in AIDL, ICDL, and FIDL. . . . . . . . . . . . . . . . . . . . . 219

A.1 Graphical representation of AIDL triggers. . . . . . . . . . . . . 243

A.2 Hierarchy of triggers. . . . . . . . . . . . . . . . . . . . 244

A.3 Example AIDL word ending with a master trigger. . . . . . . . . 245

B.1 Configuration and reference frames of a quadrotor. . . . . . . . . 248

C.1 'Level flight' template. . . . . . . . . . . . . . . . . . . . . 255

C.2 'Ascent/descent' template. . . . . . . . . . . . . . . . 256

C.3 'Level thrust acceleration/deceleration' template. . . . . . . . . . 256

C.4 'CAS/Mach climb' template. . . . . . . . . . . . . . . . 257

C.5 'Mach/CAS descent' template. . . . . . . . . . . . . . . . 257

C.6 'Constant path ascent/descent' template. . . . . . . . . . . . . 258

C.7 'Constant path constant speed ascent/descent' template. . . . . . 258

C.8 'Ascent acceleration with ESF' template. . . . . . . . . . . . . . 259

C.9 'Clean configuration' template. . . . . . . . . . . . . . . . . 259

C.10 'Scheduled configuration' template. . . . . . . . . . . . . . . . 260

C.11 'Geometric path waypoint' template. . . . . . . . . . . . . . . . 260

C.12 'Hover' template. . . . . . . . . . . . . . . . . . . . . . 261

C.13 'Circle around' template. . . . . . . . . . . . . . . . . . . 262

C.14 'Level' template. . . . . . . . . . . . . . . . . . . . . . . . . 262

C.15 'Ascent/Descent' template. . . . . . . . . . . . . . . 263

C.16 'Direct-to point' template. . . . . . . . . . . . . . . . . . . 263

C.17 'Face-to point' template. . . . . . . . . . . . . . . . . . . 264

C.18 'Face forward' template. . . . . . . . . . . . . . . . . . . 265

C.19 'Hold orientation' template. . . . . . . . . . . . . . . . 265 


\section{Chapter 1}

\section{Introduction}

Since the first manned heavier-than-air flight, aviation has come a long way. The evolution of the aircraft is perhaps the most apparent. In 1903, the Wright brother's Flyer I took off with a weight of $340 \mathrm{~kg}$ [1] and only one pilot on board. Nowadays, the Airbus A380 can take off with a weight of 575 tonnes, carrying up to 850 passengers [2]. In between, there were a great number of advancements, two of which were arguably the most influential: First, the structural revolution in the $1930 \mathrm{~s}$, favoured from the the transition from wood-and-fabric to all-metal airframes [3]. And second, the widespread use of the new, more comfortable, and much faster jet engine in commercial aviation starting in the late 1950s [2]. Today, the airframe and the engines of an aircraft remain its most important and costly items [4].

The evolution of air traffic control (ATC), although less apparent, has also been significant. Until the 1930s there was little need for organized ATC, and traffic avoidance was accomplished using the practice of 'see and be seen'. This practice required a minimum visibility conditions and a set of rules that are now known as the visual flight rules (VFR) [5]. With the development of instrument flying and the use of ground-based radio navigation aids, aircraft became capable of flying with no outside visual reference. This made flying through fog or during nighttime fairly common, conditions under which the VFR were inadequate. In 1936, the instrument flight rules (IFR) established how pilots should fly under these low-visibility conditions [5]. The air traffic control officers (ATCOs) were responsible for separating aircraft flying under IFR. Using radio communications, pilots transmitted their position to ATCOs, which might respond by issuing instructions to the pilot if a potential conflict was detected. Before the flight, pilots were also required to file an instrument flight plan, containing information such as the departure time, the intended route, and the cruising speed and altitude.

The procedures used in ATC remained largely the same for the next 20 years, but the number of operating aircraft grew by the thousands every year. The tracking method used in ATC at the time, based on pilot reports, was insufficiently accurate to handle the growing traffic. The ATC system was completely overloaded, causing substantial delays in congested areas. In the 1950s, radars began to be installed, providing more accurate tracking, thus allowing to reduce the distance between aircraft. Later, in the 1960s, computerized systems were 
introduced to automate many of the functions of the ATC system and reduce the ATCOs' workload. However, these systems were slowly deployed due to lack of funding, and the ATC system still couldn't cope with the growing demand.

The use of computer systems to automate tasks wasn't only applied to ground-based ATC systems. By the 1950s, many aircraft relied on electronic systems for their operation, and the term 'avionics' - a combination of 'aviation' and 'electronics' - was already widely used to refer to those systems. Avionic systems at that time included voice communication radio systems, navigation systems - using radio navigation aids such as VHF omnidirectional range (VOR) and distance measuring equipment (DME) - , and soon they would also include transponders to communicate aircraft state information to the ATCOs. The avionics technology evolved rapidly, driven by the need to reduce costs, improve safety, and meet ATC requirements [6]. With the introduction of the first flight management systems (FMSs) in the early 1980s, the workload of the flight crew was significantly reduced. Several tasks such as navigation, guidance, and trajectory optimization were increasingly automated. The FMS also assisted in the flight planning task. Avionic systems have evolved to become a critical item in modern commercial aircraft, and they are the third most costly item in an aircraft, after the airframe and the engines (about $10 \%$ of the total cost $[5,6]$ ).

Some new ATC techniques introduced in the 1980s, such as flow control, helped to reduce airspace congestion. In order to take advantage of the continuously improving capabilities of aircraft's navigation systems, the required navigation performance (RNP) concept was developed. RNP specifies the navigational capabilities necessary for operation within a defined airspace, without relying on any particular equipment. This concept eased the adoption of emerging technologies, such as global navigation satellite systems (GNSSs). GNSSs, and in particular the global positioning system (GPS), have contributed to increase the accuracy of aircraft's navigation, and new procedures have been introduced to benefit from this improvement in performance.

Despite all the upgrades in ATC, developments on board aircraft have outpaced those in ATC centres. Aircraft are equipped with advanced navigation systems, but still flying routes that were defined, in some cases, in the 1940s and 50s [7]. This lag in ATC has prevented modern avionics to be fully exploited in order to achieve more efficient flights and more airspace capacity.

\subsection{Future challenges in ATM}

In order to cope with the future demands of air transportation, it will be necessary to shift from current tactical ATC to a more strategic air traffic management (ATM). As defined by the International Civil Aviation Organization (ICAO) [8]:

ATM is the dynamic, integrated management of air traffic and airspace — safely, economically, and efficiently — through the provision of facilities and seamless services in collaboration with all parties.

The Global ATM Operational Concept [8] aims to increase the capacity and improve the safety levels of the ATM system. More efficiency is expected to be 
obtained by giving more flexibility to airspace users (i.e., airlines, pilots, and operators) to fly the trajectories that better suit their needs. Regional initiatives, such as the Single European Sky ATM Research (SESAR) in Europe or the Next Generation Air Transportation System (NextGen) in the United States, have further developed this operational concept.

\subsubsection{Trajectory-based operations}

Under the new paradigm, a trajectory must be agreed between the airspace user, the air navigation service provider (ANSP), and the airport before every flight. The agreed trajectory, called business trajectory (or mission trajectory for military flights), must then be flown by the user and facilitated by the ANSP and airport [9]. This concept is known as trajectory-based operations (TBOs).

The business (or mission) trajectory can be generated months before the flight. It is the responsibility of the airspace user to generate the initial trajectory, which is then shared with other members of the ATM community. A collaborative decision making (CDM) process begins, during which the airspace user may be requested to adjust the trajectory to adapt to the network constraints. An agreement on the business (or mission) trajectory must be reached before departure time, and it becomes the goal to be achieved. These trajectories are described as a four-dimensional (4D) flight path of the aircraft through space and time [5], and must be followed with a required precision. However, business/mission trajectories may still change during flight if the previously agreed trajectory cannot be achieved, or if the trajectory must be modified by ATC. A new CDM process may start in this case to agree the new trajectory, unless it is a time-critical ATC or pilot tactical decision.

The trajectory generation and negotiation processes will be automated to a great extent. Therefore, these processes will require establishing a common language in which the members of the ATM community can express business/mission trajectories and network constraints. This language must be easily processable by a computer system, but it also must be readable by a human operator supervising the process.

Moreover, trajectory predictors will become an essential function of any airborne or ground-based automation tools. Also, the more precise the trajectory predictors, the more reliable business (or mission) trajectories will be, and amends of the trajectory during flight will be less frequent.

\subsubsection{Unmanned aircraft}

Future ATM will have to accommodate new uses of airspace, such as unmanned aircraft (UAs). UAs are flown without a pilot on-board, and they are either remotely controlled or programmed and fully autonomous [8]. The term UA has recently replaced the term unmanned aerial vehicle (UAV). Also, in order to reflect that a UA is usually a part of a larger system including groundbased stations, operators, and communications, the term unmanned aircraft system (UAS) has been introduced [10].

Flight operations of UASs are expected to grow, both in their civil and military applications. These systems are usually associated with 'dull, dirty, 
or dangerous' tasks, but the fact is that, in some cases, UAS are simply the safest or most cost-effective way to complete that task. Uses of UASs include border patrolling, search-and-rescue, emergency management, wildfire suppression, scientific research, transport of materials [11], and other industrial applications [12].

In order to enable a shared airspace for manned and unmanned aircraft, technology must ensure a transparent operation regardless of the type of aircraft. This will require UAs to fly under the TBO paradigm, which should be extended to the whole UAS.

\section{$1.2 \quad$ Formal languages}

When control towers and aircraft began to be equipped with radio communication systems, ATCOs and pilots could communicate with each other by voice. The lack of an agreed phraseology made it difficult to issue precise instructions to pilots, who sometimes couldn't understand what they were being asked to do [5]. Currently, there is a standard phraseology for these voice communications [13].

Natural languages, that is, the languages spoken by people, are often ambiguous, redundant, and not literal. They have evolved naturally over the years, and follow no design principles. This makes them very difficult for a computer program to understand, and inadequate for transmitting precise and unequivocal information. A standard phraseology, while it allows the transmission of precise instructions or information, it falls short for the requirements of future ATM. The TBO paradigm and the associated CDM process will require much more information to be shared, including business (or mission) trajectories, constraints, clearances, etc. For this purposes, formal languages are far more adequate.

Formal languages can be designed to suit the specific needs of a particular domain, avoiding many of the problems of natural languages. A formal language is a (possibly infinite) set of words $L$, where the words in $L$ are formed from an alphabet $\Sigma$ (i.e., a set of symbols) and a grammar (i.e., rules to form words using the symbols in the alphabet). Formal languages are used to represent information in many different domains, including mathematics (mathematical notation), chemistry (chemical formulas), and computer science (programming languages). The standard phraseology currently used in ATC can also be seen as a formal language.

There is an extensive theory behind formal languages [14] that allows designing a language for describing any kind of information in a way that is unambiguous and easily processable by a computer program. Several languages developed using this theory will be described along this thesis.

\subsection{Problem statement}

The future ATM will rely heavily on the sharing of information among different actors, and the exploitation of such information by automatic processes. This thesis explores the use of formal languages for the representation of 
trajectory-related information, and the applications that such languages could provide to ATM.

It should be possible to completely describe an aircraft's trajectory using formal languages during all phases of flight, from push back from the gate to the arrival at the gate ${ }^{1}$ (including taxiing at the departure airport, take-off, climb, cruise, descent, and taxiing at the destination airport). One formal language has already been developed for describing fixed-wing aircraft trajectories [15, 16], the aircraft intent description language (AIDL). Since this language can only be used to describe trajectories of that type of aircraft, it will be often denoted fixed-wing AIDL (FW-AIDL) in this thesis. The FW-AIDL will be used as a basis for the development of other languages that provide additional features via the addition of new semantics.

Uncertainty is a key aspect in many of the problems related to ATM. However, the FW-AIDL, as well as the languages developed in this thesis - and their associated applications - will focus on the deterministic variants of such problems.

\subsection{Summary of this work}

In this thesis, several formal languages are presented, which allow describing a trajectory with an arbitrary level of detail. Some of these languages, as well as the associated prototypes, have been developed in collaboration with Boeing Research \& Technology Europe (BR\&TE).

The languages for describing trajectories have several applications, including negotiation, trajectory prediction, mission management, conflict detection and resolution, etc. In the context of future ATM, trajectory prediction has a high relevance. Thus, this thesis makes a special emphasis on the applications of the aforementioned formal languages to trajectory prediction. Applications to mission management are also explored.

The contents of this thesis are organized in five parts, which are outlined next.

Part I contains literature reviews covering several areas. A survey of the different means used to communicate trajectory-related information is included in chapter 2. Then, chapter 3 surveys the trajectory predictors available in the literature. Finally, chapter 4 contains a brief survey of the architecture and algorithms used in mission management, including the computation of shortest paths between locations.

The definition of the formal languages used for describing trajectory-related information is included in part II. Most of these languages are arranged in a hierarchy of languages, which is presented in chapter 5 .

At the foundations of this hierarchy are the FW-AIDL and the quadrotor AIDL (QR-AIDL). The QR-AIDL is a variant of the FW-AIDL that can be used for describing trajectories of quadrotor aircraft, and it is developed in chapter 6 . These two languages are derived from the equations of motion of

\footnotetext{
${ }^{1}$ In the case of UAS, airport surface operations may be irrelevant, and only the phases when the UA is airborne need to be described.
} 
the corresponding type of aircraft, so they provide a mathematically sound and unambiguous description of trajectories.

The intent composite description language (ICDL), defined in chapter 7 , is built on top of those languages, providing an additional layer of abstraction. In this language, trajectory descriptions are constructed as a composition of simple manoeuvres, also allowing some elements of the trajectory to be defined while others are left unspecified.

Chapter 8 defines the flight intent description language (FIDL), a language that provides a level of abstraction similar to that of a flight plan (a description of the flight that must be defined prior to the flight, like the business trajectory). The FIDL allows describing different parts of the trajectory with an arbitrary level of detail, as it inherits the properties of the ICDL, plus it provides new semantics for the description of constraints and objectives.

A language for describing missions involving several aircraft is defined in chapter 9. This language, the mission intent description language (MIDL), is based on the FIDL and allows describing tasks, constraints, and objectives affecting the mission.

The operations carried out by vehicles on airport surface can also be described using a formal language, the surface movement description language (SMDL). This language is derived from the clearances issued by ATCOs during airport surface operations.

Part III discusses some of the applications of those formal languages. The concept of trajectory language processing engine (TLPE), an ATM function or sub-function whose main input and output are the formal languages described in this thesis, are introduced in chapter 11. This chapter also provides multiple examples of ATM functions formulated as TLPEs. In particular, a description of a trajectory predictor is provided, which is built as an aggregation of TLPEs. More details on the critical algorithms used in that trajectory predictor, the generation of the horizontal and vertical profiles, are given in chapter 12 . The applications of TLPEs are not limited to ATM. Some applications of this concept to UASs are also discussed in this part of the thesis. For example, a trajectory computation engine (TCE) designed to compute QR-AIDL words and generate sampled trajectories of quadrotor aircraft is presented in chapter 13. Additionally, chapter 14 expresses the mission planning problem as a TLPE, analysing in detail one of its derived problems: computing paths between two locations in an urban environment.

The conclusions of this thesis, providing future lines of work and a list of contributions, are included in part IV. The bibliography used throughout the thesis is also listed in this part.

Finally, part $\mathrm{V}$ contains the appendices of this thesis. A short description of the FW-AIDL is provided in appendix A. Appendix B defines the equations used in chapter 6 to model the flight of a quadrotor. A comprehensive list of the ICDL templates used in the examples along this thesis is included in appendix C, along with a brief description of each of them. Lastly, appendix D provides a complete formal specification of some of the languages defined in part II. 


\section{Part I}

\section{Background and Literature Survey}





\section{Chapter 2}

\section{Specification of flight-related data}

This chapter discusses the different means used to describe a flight that have been used in the literature. Some of these means are the precursors of the languages presented in part II of this thesis.

Sharing flight information is a fundamental requirement in modern ATM. Flight information sharing may occur prior to the flight (e.g., flight plan filing) or during the flight (e.g., a clearance issued to the pilot), and the information may be shared from air to ground, ground to air, air to air, and ground to ground. In fact, the means to express flight-related data have existed since the very beginnings of ATC. Communication of flight information has evolved from voice commands [13] (still in use today) or handwritten forms (such as flight strips [5]) to much more complex representations that allow higher levels of precision and automated processing. Digital transmission of information between the ATCO and the flight crew has been made possible with the controller-pilot data link communications (CPDLC) [17], which allows text messages to be sent using the phraseology of voice communications.

More advanced communications are under development. In the last years, the Radio Technical Commission for Aeronautics (RTCA) special committee 214 [18] and European Organisation for Civil Aviation Equipment (EUROCAE) working group 78 [19] have been tasked to develop the interoperability requirement standards for a set of advanced communications services. These standards include the $4 D$ trajectory data link (4DTRAD) [20], which enables air-to-ground and ground-to-ground synchronization of precise flight information, and the flight object interoperability proposed standard (FOIPS) [21], a format that allows sharing information about a single flight.

Regardless of the technology used for communication, a flight can be described by either specifying the trajectory (or a prediction of the trajectory) or the intent. Specifying a trajectory consists on describing the evolution of the state of the aircraft during a certain time. The state of an aircraft must contain at least a description of the aircraft's position, but may contain many other variables. The intent, on the other hand, provides information on the aircraft's behaviour, rather than its trajectory. According to [22], the intent information 
'describes the notional path and/or constraints the aircraft will follow in the future', and it 'may be a sequence of control instructions, a flight plan, or a simple projection of the velocity vector'. In other words, the intent information provides a description of an aircraft's trajectory, but it's not the trajectory itself [16]. There are several different levels of detail that can be used to express intent information. The aircraft intent provides the most precise description of an aircraft's behaviour. Other expressions of intent, such as the flight intent and the mission intent provide higher-level descriptions, each of them serving different purposes. The flight intent can be seen as an equivalent of the flight plan (at least in the precision of the flight description), whereas the mission intent provides more abstract descriptions of aircraft trajectories.

In both cases, the amount of information in a flight description will depend on the needs of the particular application and the information available at some particular time. Therefore, different means for describing a flight may be the most suitable depending on the context.

In addition to descriptions of the flight itself, this chapter discusses the available means for representing other types of flight-related data, including strategic and tactical constraints, objectives, and trajectories of aircraft and other vehicles on airport surface.

\section{$2.1 \quad$ Specification of trajectories}

In ATM, the trajectory of an aircraft can be specified by describing the evolution of the aircraft's state over time. The trajectory may be partial, if it only provides information for some portion of the flight, or a complete trajectory, if it provides information for the whole flight. Different levels of detail may also be provided by varying the number of state variables included in the trajectory.

In practice, trajectories are specified mathematically by providing a time-ordered sequence of aircraft states. Each aircraft state contains the values of the state vector of the aircraft, sampled at a particular time. Among the state variables, it is possible to find geometric (position and attitude), kinematic (linear and angular speeds), and kinetic (forces and moments) information. The samples may also contain other information such as control variables or environment variables (temperature, pressure, wind).

Some of the most simple representations used in the literature include a two-dimensional (2D) position in the vertical plane $[23,24]$ or in the horizontal plane [25-28]. The former are useful in the context of optimization in the vertical profile, while the latter can be used for optimizing traffic. More common are representations of the trajectory that include a three-dimensional (3D) position [29-31], most of them including additional state information [32-37]. This additional information is usually related to the orientation of the aircraft - using pitch, yaw, and roll angles —, its speed — as a Mach number, true airspeed (TAS), or calibrated airspeed (CAS) - , flight path angle, heading angle, aircraft mass, engine thrust (or thrust settings), lift coefficient, load factor, etc. Some of these variables depend on the weather conditions, which may be required to complete the information in the trajectory. 

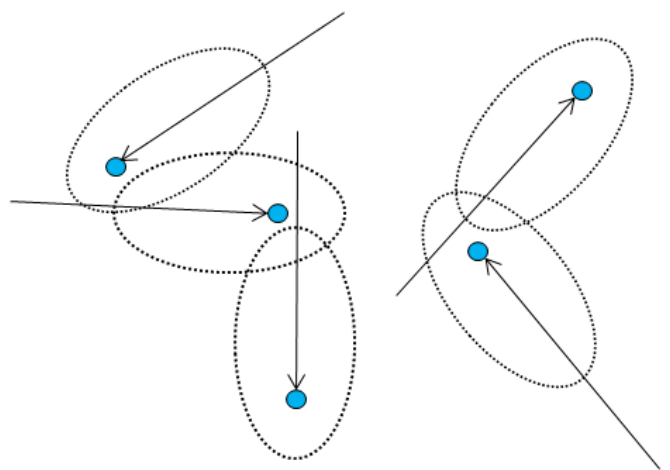

Figure 2.1: 2D representation of ellipsoids describing uncertainty of individual trajectories.

In SESAR and NextGen, the state vector (denoted trajectory vector) has a minimum four attributes: time, latitude, longitude, and altitude [38].

Trajectories, especially when they represent a prediction of a future flight, may also have to represent uncertainty in some way. Uncertainty may come from a variety of sources that are studied in [39]. Its representation in a trajectory is usually based on the tube-in-space concept [29], which defines a trajectory as a 4D tube. That is, at any time, the aircraft's position isn't univocally defined, but it is defined to be within a $3 \mathrm{D}$ ellipsoid (see figure 2.1). The $4 \mathrm{D}$ tube is the result of moving said ellipsoid with time. Related approaches are used in [40] and $[41]$.

\subsection{Specification of aircraft intent}

The aircraft intent provides information on an aircraft's future behaviour. Usually, the term aircraft intent is associated with a high-detail, low-level description of an aircraft's trajectory. However, this term has been given different definitions. The ICAO defines the aircraft intent as [42]:

Information on planned future aircraft behaviour, which can be obtained from the aircraft systems (avionics). It is associated with the commanded trajectory and will enhance airborne functions. The aircraft intent data correspond either to aircraft trajectory data that directly relate to the future aircraft trajectory as programmed inside the avionics, or the aircraft control parameters as managed by the automatic flight control system. These aircraft control parameters could either be entered by the flight crew or automatically derived by the flight management system.

According to this definition, the aircraft's trajectory itself can be considered a representation of the aircraft intent. This approach was used in the INTENT Project $[43,44]$, which defined the aircraft intent simply as 'the path in time- 
space that the aircraft intends to fly', and where the aircraft intent consisted on predicted trajectories for the next 5 to 20 minutes.

However, the other type of aircraft intent data, related to the control parameters, can be of special interest in trajectory prediction. In an attempt to improve interoperability among different trajectory predictors, the processes common to any trajectory predictor were identified in $[38,45]$. Among these elements is the trajectory engine, which computes a trajectory from some unambiguous description of the aircraft's behaviour, denoted flight script or behaviour model. According to $[16,46]$, the aircraft intent 'specifies how the aircraft is to be operated during the time interval for which a computed trajectory is required. The aircraft intent must be formulated in such a way that the ensuing aircraft motion is unambiguously determined'. This definition makes the aircraft intent a good candidate to become the input of the trajectory engine (i.e., to serve as the flight script or behaviour model), and an effort to develop a common standard for sharing aircraft intent [47] concluded with the development of the aircraft intent description language (AIDL).

\subsubsection{Aircraft intent description language}

The AIDL $[15,16,47,48]$ is a formal language developed by BR\&TE to describe aircraft intent information. This language was designed to be generic, so it can describe any aircraft behaviour that is meaningful in ATM, and rigorous, so that a behaviour described in this language is consistent and unambiguous. The language provides a set of instructions, which model basic commands, guidance modes, or control strategies at the disposal of the pilot or the FMS to operate the aircraft. Each instruction closes one degree of freedom (DoF) of the aircraft motion, until a condition triggers its deactivation. Instructions constitute a metamodel of all the possible flight commands and guidance modes that can be modelled by trajectory predictors. Additionally, there are a number of rules that govern how instructions can be arranged to close all the DoF of the aircraft motion. The language is derived from the mathematical analysis of the trajectory engine, based on the theory of differential algebraic equations (DAEs) [16]. This analysis proved that a valid AIDL word (i.e., an aircraft intent expressed in the AIDL) contains all the necessary and sufficient information to compute a trajectory given any set of meaningful initial conditions. Numerous references to this language will be found along this thesis. Thus, a more detailed description of this language is provided in appendix A.

\subsubsection{Other specifications of aircraft intent}

There are other specifications in the literature that can be used to describe aircraft intent.

The GenProf scripting language [49] developed at the NASA Ames Research Center allows expressing vertical constraints in a trajectory. This language provides three different types of element - anchor, profile, and stop elements-, and a series of rules for combining these elements to describe meaningful flights. The anchor represents the initial conditions of the aircraft trajectory. It consists of an altitude, a speed, and a distance along the horizontal path. The stop 


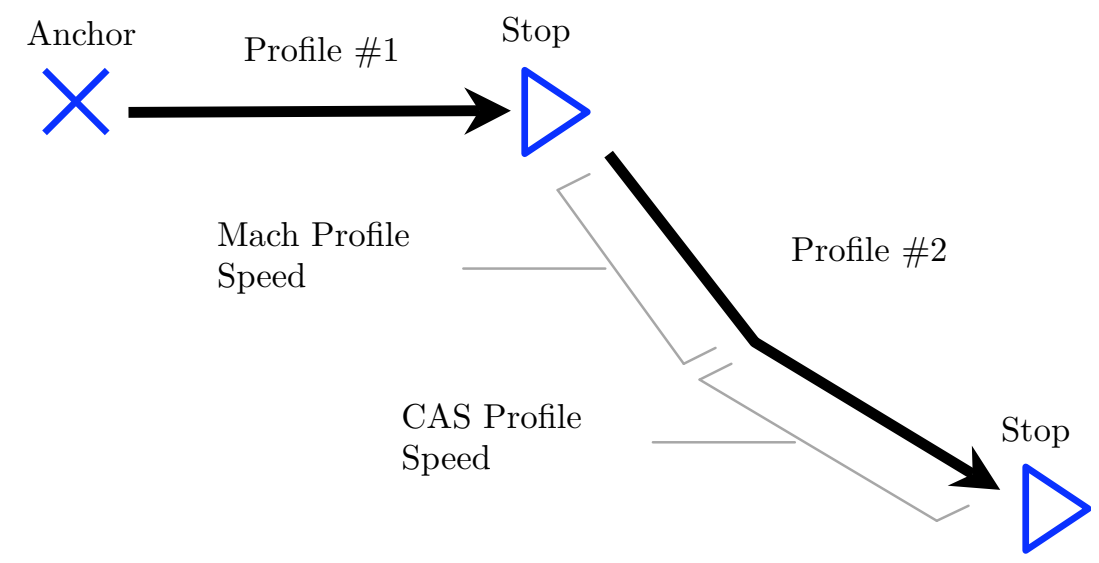

Figure 2.2: Graphical representation of the GenProf expression.

element represents the condition that terminates the trajectory segment. The condition can capture a specific value for a variable that can be related to altitude, time, speed, or distance along the horizontal path. More complex conditions can be formed by using 'and' $(\wedge)$ and 'or' $(\vee)$ boolean operators. Finally, the profile element describes how a trajectory segment is built from its beginning (triggered by an anchor or stop element) to its end (triggered by a stop element). A profile is specified by defining two parameters, which dictate the equations of motion used to compute a trajectory. Among the available parameters, the following can be found: path angle, vertical speed, throttle setting, and Mach-CAS profile.

A GenProf expression starts with an anchor element defining the initial conditions. Then, a profile and stop elements must follow, but more profile-stop pairs can be chained together to build more complicated vertical profile expressions. An example GenProf expression can be found in table 2.1, describing a cruise segment followed (after 20 minutes of flight) by a Mach-CAS descent to 18,000 feet. This example is also depicted in figure 2.2.

\begin{tabular}{c|c|c|c|c} 
Anchor & Profile \#1 & Stop \#1 & Profile \#2 & Stop \#2 \\
\hline \hline $\begin{array}{c}\text { 33K ft alt. } \\
\text { Mach 0.8 }\end{array}$ & $\begin{array}{c}0^{\circ} \text { path angle } \\
\text { Mach 0.8 }\end{array}$ & 20 min. & $\begin{array}{c}\text { Mach 0.8/280 kt CAS } \\
\text { Idle engine control }\end{array}$ & $18 \mathrm{~K}$ ft alt.
\end{tabular}

Table 2.1: Simple GenProf expression

The common interface for trajectory computation (CITRAC) [50], developed by the Centre d'Études de la Navigation Aérienne (CENA), aimed to define a standard input and output for different trajectory predictors, each with its own purpose and context. The input data defined in CITRAC can be used to describe aircraft intent. In this format, the aircraft intent is provided as two separate profiles: the horizontal profile and the pilot profile. The horizontal profile is defined as a list of segments containing the manoeuvre to be executed, which include the following: direct, radial, take/keep heading, holding pattern, follow DME arc, etc. Each of the segments also contains an end condition that terminates the segment, i.e., a boolean condition regarding the horizontal posi- 


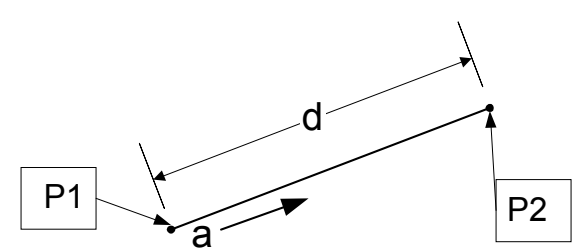

(a) Line segment.

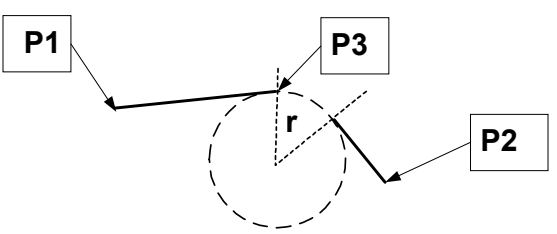

(b) Turn with turn radius.

Figure 2.3: Examples of path object shapes.

tion of the aircraft, its altitude, or a specified time. Finally, segments may have associated constraints, which limit the altitude, speed, rate of climb/descent, or time at the end of the segment. The pilot profile defines a list of modes of operation to be followed sequentially by aircraft during the flight. A mode of operation defines the speed, thrust, and configuration of the aircraft until a condition is met to go to the next step.

Path objects [51, 52], developed at the Center for Advanced Aviation System Development (CAASD) of the MITRE Corporation, can describe the horizontal profile of an aircraft's trajectory using a series of primitives. These primitives define the most common shapes flown by FMSs, independently of their location, orientation, size, etc. By providing this information, the primitives are particularized to generate the path object. The primitives in $[51,52]$ include lines (figure 2.3a), turns (figure 2.3b), arcs, and holding patterns. However, path objects cannot describe any horizontal path as it is limited by the available primitives, and provides no means to specify the vertical profile.

\subsection{Specification of flight intent}

The flight intent provides a higher-level description of a flight. In most cases, this description is at the same level of detail as the flight plan is. It forms the basis for an ATM system agreement, while the aircraft intent forms the basis for the confirmation of compliance with the agreement [42]. There are also different definitions for the flight intent, as there were for the aircraft intent.

The ICAO defines the flight intent as [42]:

The future aircraft trajectory expressed as a 4D profile until destination (taking account of aircraft performance, weather, terrain, and ATM service constraints), calculated and "owned" by the aircraft FMS, and agreed by the pilot.

This definition doesn't match the description of flight intent given above, as the level of detail is much higher than a flight plan. In fact, according to this definition, the flight intent is a completely defined $4 \mathrm{D}$ trajectory, which also doesn't match with the definition of intent given at the beginning of this chapter. It has also been argued that this definition requires an operational concept very different from today's modes of operation, and not applicable in the short to medium term future [47]. Alternatively, as defined in [16, 46, 53], the flight intent is 'a description of the key operational requirements and constraints that 
must be fulfilled by the computed trajectory (e.g., intended route, operator preferences, standard operational procedures, ATC constraints, etc.)'.

Under this definition, more flight specifications fall in the category of flight intent. In current air traffic operations, the ICAO Flight Plan is the standard way for describing the aircraft's intended trajectory.

\subsubsection{ICAO Flight Plan}

The ICAO Flight Plan [13] is a standard document that must be filed with the civil aviation authority prior to any flight ${ }^{1}$. It contains detailed intentions on a particular flight.

The information in the flight plan directly related to the trajectory includes the origin and destination aerodromes, with a list of alternate destinations, the approximate time of departure, the total estimated elapsed time (for complete flights, this is the duration of the flight), the cruise altitude and speed, and the route. The route is a sequence of air traffic service (ATS) routes (defined as a coded designator) or direct flights (denoted 'DCT') to a significant point (defined using a coded designator, longitude and latitude, or a bearing and a distance from a reference point). If an altitude or speed change is planned at one of the points in the route, this point must be explicitly included, together with the target altitude or speed.

The flight plan also includes miscellaneous information related to the aircraft's equipment and capabilities, the flight rules - IFR or VFR -, type of flight, type of aircraft, fuel endurance, supplementary information regarding the people on board, etc.

\subsubsection{Other specifications of flight}

One of the most simple representations of a flight that meets the definition of flight plan given above would consist of a pair of initial and final flight conditions. In some cases, the initial and final conditions are specified as 2D points $[25,54]$. The initial and final conditions may also include state or control variables such as the flight path, heading angles, mass, velocity or a more sophisticated condition formulated by the combination of several state variables $[24,29,31,32,36]$.

Intermediate requirements on the flight (in addition to initial and final conditions) can be added to form more detailed specifications. For example, in [26, 27], the flight is described as a sequence of $2 \mathrm{D}$ waypoints. These waypoints can also include other information such as the altitude or course [33]. Other types of intermediate requirements are also allowed. According to [38], the flight intent may contain objectives from the aircraft operator and constraints from the aircraft characteristics, from airport and airspace resources, and from safety requirements. Some of these constraints and objectives are described in more detail in section 2.3.3.

Flight specifications also exist in other contexts, such as UASs. There is no standard specification in this field, and several manufacturers and organizations

\footnotetext{
${ }^{1}$ Some authorities, such as the Federal Aviation Administration (FAA), allow the use of a domestic format flight plan. These are usually simplified versions of the ICAO Flight Plan.
} 
use their own ad-hoc solutions. Commercial off-the-shelf (COTS) autopilot systems for UASs usually use 2D or 3D waypoints to define the flight plan. Some authors have provided more flexibility and enabled more complex specifications by allowing iterations and forks in the flight plan $[55,56]$.

\subsubsection{Additional flight information}

Besides flight plan specifications, there are other kinds of information tightly related to the flight that must be taken into account when planning a flight and during trajectory prediction. This section focuses on two particular types of information: constraints and objectives.

Constraints provide additional means to specify requirements on the flight. They can be related to the restrictions in the airspace, the limitations in aircraft performance, or the time.

Considering their scope, airspace constraints may be classified in long-term (strategic) and short-term (tactical) constraints. Strategic constraints are permanent restrictions on the airspace (or, at least, constant for the duration of the flight), and include the constraints in the aeronautical information publication (AIP). Some digital formats for storing AIP information are available, such as the aeronautical information exchange model (AIXM) [57] or ARINC 424 [58]. These constraints are usually expressed through a domain of application (DoA) -i.e., the region of space where the constraint must be met-, and a restriction in the aircraft state - i.e., the condition that must be met, usually related to speed or altitude. DoAs may be restricted to simple geometrical shapes [26] or arbitrary shapes [37]. Sometimes, DoAs are defined by elements of the airspace structure, such as legs, routes, significant points, or 3D volumes.

Tactical constraints, usually denoted clearances, are issued by ATCOs when the traffic conditions require so. They consist of guidance instructions to be followed by the flight crew, provided they are safe and feasible. Clearances may be transmitted to the flight crew via voice communications using a standard phraseology [13] — e.g., 'maintain flight level 40 until further advised', 'turn right heading 080', etc.-

Aircraft performance constraints may consist of restrictions applied to certain state variables (e.g., speed, lift, or thrust) [29, 34]. This approach is common in control and guidance applications, but in ATM trajectory prediction the most common approach is using an aircraft performance model (APM). APMs provide functions that model several effects affecting a particular type of aircraft (aerodynamic forces, thrust, and fuel consumption). The models provided by the base of aircraft data (BADA) [59] are widely used.

Time constraints, expressed as a required time of arrival (RTA) to a specific point, are used to reduce uncertainty in the along-track dimension. RTAs may be specified as time intervals [60]. In other cases, the RTA is given as a target time that is interpreted as an objective, rather than a constraint [29].

Objectives represent optimization criteria for all or a portion of the flight. This information that can be used in trajectory optimization processes to minimize (or maximize) a certain cost (or merit) function evaluated over all or part of the trajectory. Usually, objectives are related to fuel consumption $[29,31$, $34]$, flight duration [26, 36, 54], or a trade-off between these two factors [61]. 
Other optimization objectives involve airline direct operating costs [25], deviation with respect to a reference track [23,37], or passenger comfort by reducing the magnitude of manoeuvring accelerations [27].

\subsection{Specification of mission intent}

Another important category of flight-related information is the mission. A mission involves several aircraft working to complete a number of tasks. Every aircraft may have a different payload and be able to perform some types of task, e.g., an aircraft equipped with a camera can perform surveillance tasks, other aircraft can transport goods, etc.

In the military context, formal languages have been developed to unambiguously describe the commands that units must do to succeed at a task, such as the coalition battle management language (C-BML) [62, 63]. The C-BML can also be used to share other types of information among military units, providing situational awareness. A generalization of this language, a command and control information system (C2IS) called SocialC2IS [64], has also been used for civilian purposes. These languages, however, are intended for real-time communications, and have not been designed to describe a complete mission.

Apex [65] is a toolkit developed by NASA for creating autonomous agents. It can be used to provide autonomous mission management for UASs. The behaviour of Apex agents is specified using the procedure description language (PDL), a formal language for representing mission plans (complete or partial), among other things. This language can describe not only the tasks in the mission, but also how to achieve them using procedures. The example procedure below describes how to obtain an image from an UA:

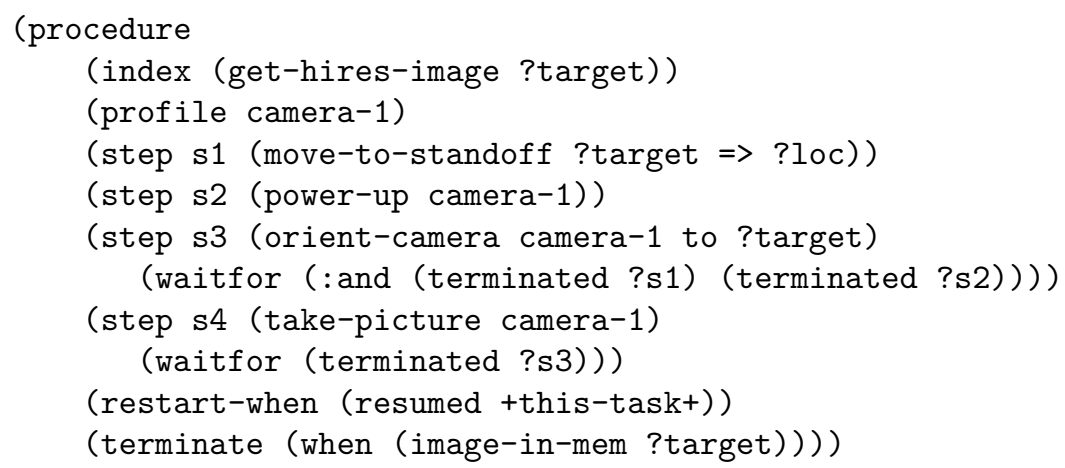

The steps in a procedure don't have to be executed in the order they appear, they can be executed concurrently unless explicitly stated otherwise. In the PDL, tasks can be recursively defined as a combination of several sub-tasks. Also, non-linear execution constructs, such as looping and branching, are included. The decision on which task is assigned to each vehicle can be left up to the resource scheduler, as long as the vehicle has the necessary payload to perform the task.

Other authors have used logics that can be used to explicitly express actions occurring along time and their effects. Examples of these logics are the temporal 


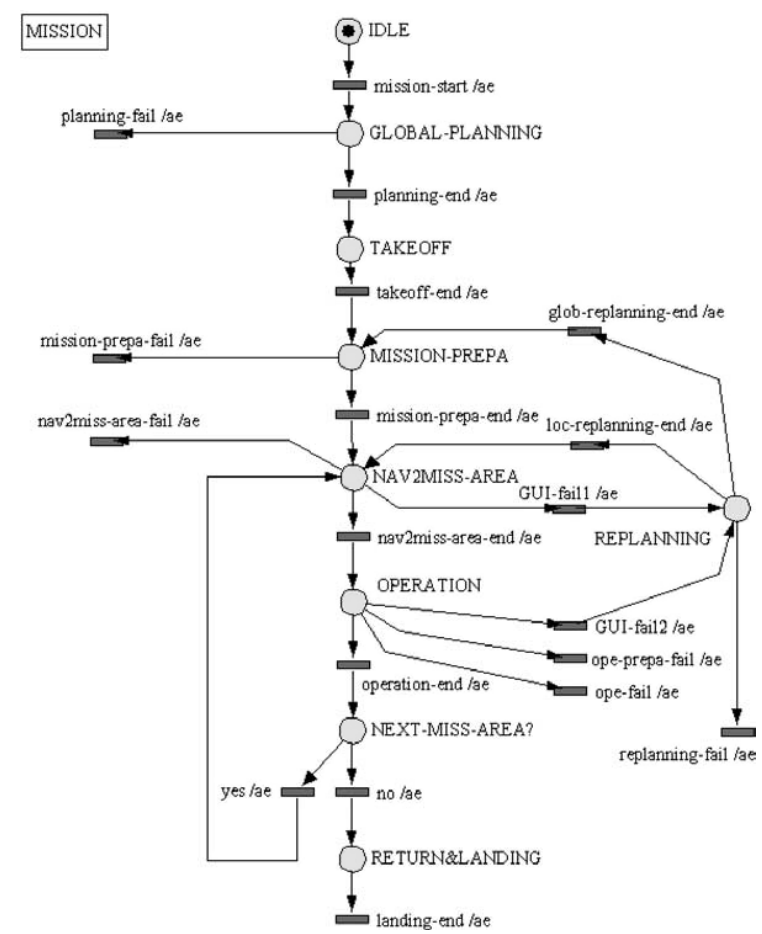

Figure 2.4: Example Petri net "mission".

action logic (TAL) [66] and the linear temporal logic language [67]. These logics result in very general specification languages, where many different kinds of mission can be defined.

Another approach consists on grouping vehicles by the role they play in the mission [68]. Roles can include surveillance, vision, short- and long-range communications, or control. The role determines the tasks a vehicle can accomplish and the privileges it can be granted. A number of tasks is then assigned to each role. Tasks can be composed of several stages, and a finite state machine (FSM) is used to represent these stages and transitions between them. FSMs are often used to model behaviour in missions of UAs [69, 70]. In particular, Santamaría [70] uses Harel's statecharts [71] as the base for his mission model.

Petri nets [72] can also be used to describe a mission. Barbier and Chanthery [73] use a hierarchy of Petri nets: a Petri net "mission" describes the general behaviour of vehicles from take-off to landing, indicating the phases or high level actions. Figure 2.4 shows an example Petri net "mission". Secondary Petri nets detail each of the phases in the mission, containing more fine-grained information on the aircraft's behaviour.

The term mission intent has already been used in [74]. In this work, the authors define the requirements for the definition of a formal language for describing mission intent. This language, still under development, models missions regarding the expected actions and their results. In addition to this language, another formal language is included to describe the payloads on-board the UAs. 


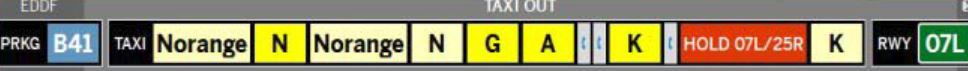

Figure 2.5: Taxi clearances on an enhanced flight deck.

\subsection{Specification of airport surface movement}

In airport surface, the traffic of aircraft and other vehicles must be managed efficiently to avoid delays. The advanced surface movement guidance and control system (A-SMGCS) aims to improve efficiency of controllers by providing improvements in several areas, including routing and guidance. Guidance methods allow the transmission of a route from the ground stations to the flight crew. This can be based on ground visual aids, such as lighting - for example, the airfield ground lighting automation system (AGLAS) [75]-, markings, and signage.

However, guidance instructions are primarily given in the form of clearances that the controller issues and the flight crew must follow. There is a fixed set of instructions that can be given as a clearance by the airport surface controller using a standard phraseology [13]. Clearances contain the identifier of the aircraft (callsign) they are issued to and, optionally, depending on the type of clearance, further information such as a runway, holding point or path identifier.

Clearances can also be represented graphically on a display in the flight deck, as shown in figure 2.5. Moreover, on-board guidance systems [76] can also provide visual aids on a display by indicating the position of the aircraft on a map together with the route to be followed, as in figure 2.6.

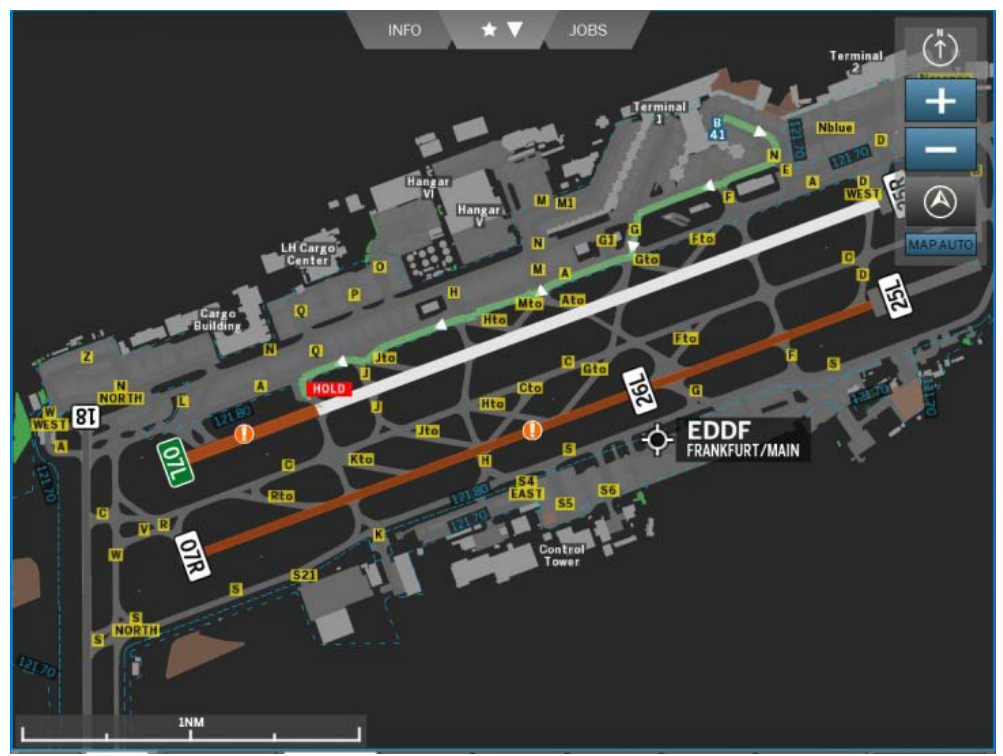

Figure 2.6: Taxi route guidance on an enhanced flight deck. 


\subsection{Final words}

This section has provided an overview of some of the formats used to communicate flight-related information. Most of these formats have been developed to describe trajectories of airborne aircraft, although the different formats have very different characteristics. Some of these formats allow providing a high level description of the trajectory, while others provide the complete state vector of the aircraft sampled in very short intervals.

Trajectory predictors can be used to generate a detailed trajectory from a high level trajectory description. A literature review on prediction of aircraft trajectories is provided in the next chapter. 


\section{Chapter 3}

\section{Prediction of aircraft trajectories}

Some of the means to express trajectory-related data discussed in chapter 2 were designed to be used as the input or output of some trajectory predictor. In this chapter, the different trajectory predictors present in the literature are described.

The main function of a trajectory predictor is the generation of a forecasted trajectory based on the available data, including the flight plan, an estimation of the current aircraft state, expected environmental conditions, mathematical models of aircraft performance, and behavioural information (i.e., estimates on how the aircraft is operated by either the pilot or the FMS to comply with the flight plan and any other constraints) [77].

The applications of trajectory predictors are manifold. They can be used by decision support tools (DSTs) to detect potential conflicts and assess different solutions to them, to assist in flight planning or re-planning, predict future sector load, simulation, research, etc. Additionally, some trajectory predictors can also be used to search for optimal trajectories given an input intent with a series of constraints, if they are capable of finding the optimal trajectory compatible with the intent.

Trajectory predictors are a key element for the enhancement of safety and capacity of airspace, and they will become even more important in future ATM. Most commercial airliners already have a FMS on board with trajectory prediction capabilities. The expected switch from a mainly tactical ATC to TBOs will make trajectory predictors an essential function of any airborne or groundbased automation tool. In a TBO environment, a trajectory must be agreed between the different actors involved in ATM. While the global system will remain human centric, actors will increasingly rely on DSTs, which must be capable of predicting and exchanging trajectories with the other members of the ATM community.

The performance requirements that different DSTs have for trajectory predictors can vary widely depending on the particular application. Therefore, multiple trajectory predictors co-exist within the ATM system with different ap- 
proaches, capabilities, response times, accuracies, and data requirements. This has led to potential interoperability issues that may limit the performance of the whole ATM system. Some recent efforts have been aimed to providing a common structure and interface for trajectory predictors in order to facilitate interoperability.

\subsection{Structure of a trajectory predictor}

The common trajectory predictor structure shown in figure 3.1 was developed by the FAA/EUROCONTROL Action Plan 16 [45]. This structure is the result of identifying common aspects among trajectory predictors, combined into a single logical structure.

This structure comprises four key processes:

- The trajectory prediction process integrates the flight script into a $4 \mathrm{D}$ trajectory, additionally using the aircraft performance information and meteorological data. The flight script can be specified using the AIDL or other unambiguous specification of aircraft intent. This process is carried out by the trajectory engine, which is highly dependant on the specification of the flight script.

- The preparation process is responsible for building the flight script from one or more higher-level sources of flight information, such as the flight plan, ATC constraints, or objectives.

- The trajectory prediction update process responds to changes in the available information by updating the flight script. Such changes may be due to new or updated constraints, weather forecasts, etc.

- The trajectory prediction export process makes the predicted trajectory data available to the trajectory predictor clients.

Note that not all the processes must be present in every trajectory predictor. The necessary processes vary for different applications, and therefore the structure presented in figure 3.1 shows the elements that may be present in a trajectory predictor. This structure has been widely accepted by the research community, as it is consistent with existing trajectory predictors, both groundbased and airborne.

A compatible model was proposed in [16]. This model, depicted in figure 3.2, divides the trajectory predictor in two components: the aircraft intent generation infrastructure (AIGI) and the trajectory computation infrastructure (TCI) [78]. The aircraft intent generation engine (AIGE) is the main process within the AIGI. This process is equivalent to the preparation process in figure 3.1, as it transforms a flight intent (i.e., a high-level flight specification) into an aircraft intent (i.e., a flight script) using other sources of information, particularly the user preferences model (UPM) and the operational context model (OCM). Similarly, the main process within the TCI, the trajectory engine (TE), is equivalent to the trajectory prediction process in figure 3.1. In order to compute a trajectory, the TE needs the APM, containing the necessary aircraft performance 


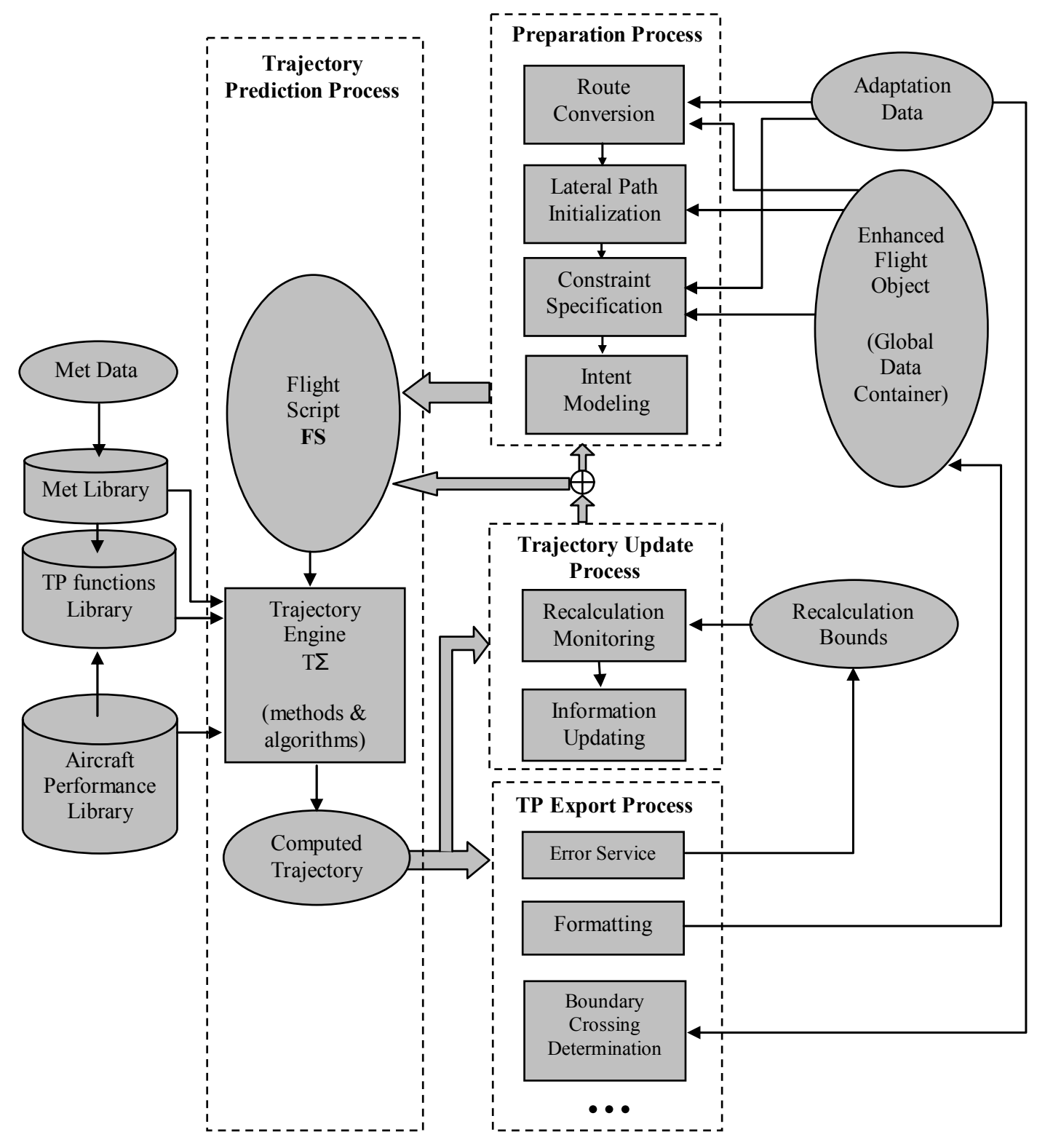

Figure 3.1: Trajectory predictor structure. 


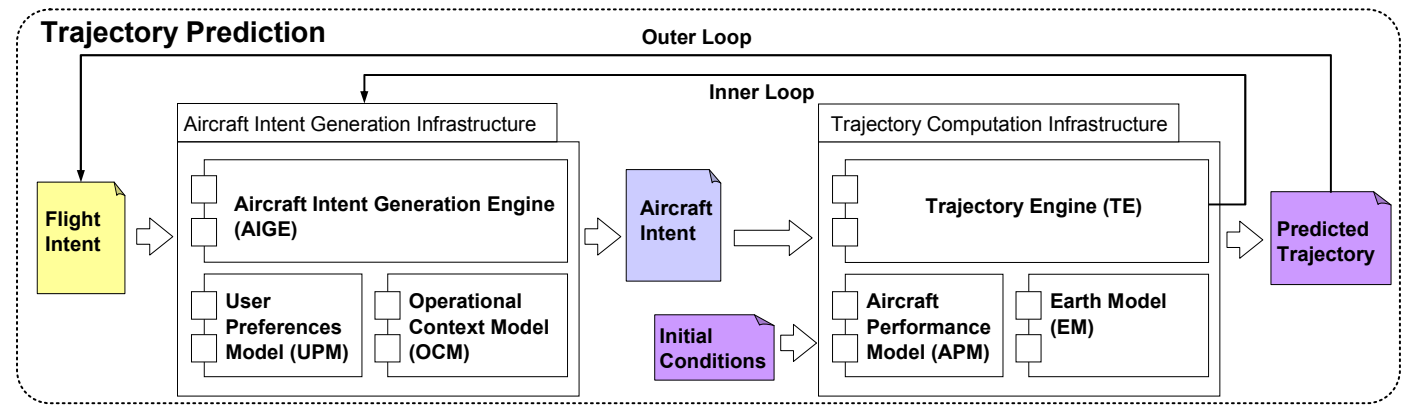

Figure 3.2: Schematic view of a trajectory predictor.

data, and the Earth model (EM), which includes atmospheric data (e.g., temperature, pressure, wind, etc.) among other information. This model can be seen as a simplification of the structure in figure 3.1, as it doesn't include the update process (which is partially represented in the inner and outer loops in figure 3.2) nor the export process. Nevertheless, this simplified model contains the two core functions of any trajectory predictor and can be used to accurately represent the main stages of trajectory prediction.

These two core functions (the trajectory prediction process and preparation process) have been addressed using different methods, which are described in the following sections.

\subsection{Models of aircraft performance}

The computation of a 4D trajectory from a flight script can be performed using a mathematical model describing the aircraft's performance. These models may belong to any of the following classes [79, 80]:

- Class A. Full dynamical models, including translational and rotational motion. These models use equations of motion of six mechanical DoFs.

- Class B. Point-mass models, including translational motion only. These models use equations of motion of three mechanical DoFs.

- Class C. Parametric models, where accelerations and speeds are modelled instead of forces.

- Class D. Fixed models, where look-up tables are used to extract the speed corresponding to an altitude range and flight phase [81].

Class A provides the highest level of fidelity, but requires the most computational load. Subsequent classes provide decremental levels of fidelity, being class $\mathrm{D}$ the oldest one and the one that provides the lowest level of fidelity. Any model in class A, class B, or class $\mathrm{C}$ is adequate for aircraft under FMS control [82].

A more detailed definition of class A and class B models will be provided in chapter 6. 


\subsection{Development of trajectory predictors}

Trajectory predictors have existed for a long time, both in ground stations and airborne, as a component of FMSs. In this section, the most relevant trajectory predictors found in the literature are described, focusing primarily on their preparation models.

\subsubsection{CTAS trajectory synthesizer}

In 1984, researchers at the NASA Ames Research Center completed the development of a trajectory synthesizer (TS) to compute fuel-efficient descents to the metering fix. The TS was later integrated in the Center-TRACON Automation System (CTAS) [83]. In the TS, the behavioural model (or flight script) is generated using a procedural approach, that is, imitating pilot flight procedures. The generation of the horizontal and vertical profiles is performed separately.

However, completely decoupling those two profiles is not possible, as the radius of each turn is coupled to the ground speed. An approximate vertical profile is used to estimate ground speeds at each turn, assuming fixed climb/descent rates and ignoring accelerations and decelerations (i.e., allowing instantaneous changes of speed). Then, the horizontal profile is generated, consisting of a series of straight lines connecting the points in the flight plan, with a turn at each point. Each turn may be an inside turn, an start-at waypoint turn, or an end-at waypoint turn (see figure 3.3). Straight lines are constructed from great circles (orthodromes), whereas turns are constructed from one or more circular arcs.

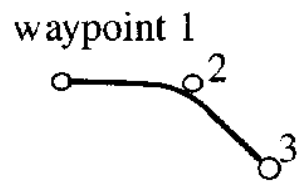

(a) Inside turn.

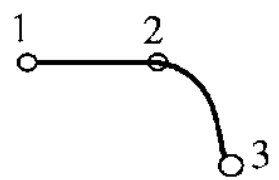

(b) Start-at waypoint.

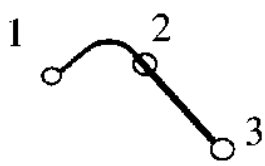

(c) End-at waypoint.

Figure 3.3: Types of turn in CTAS.

Once the horizontal profile is completed, the vertical profile is generated, consisting of a sequence of segments to be flown in a specific order. Among the three types of variables that the pilot can use to control the aircraft - engine control, speed, and vertical rate-, each segment holds two of them constant. That is, in one segment either thrust and speed, thrust and vertical rate, or speed and vertical rate, are constant. Transitions from one segment to the next occur when a capture condition is met. The capture condition usually consists on one of the non-constant variables reaching a desired value.

The vertical profile is generated by concatenating three pre-defined templates: one ascent, one cruise, and one descent template. Templates contain a sequence of segments representing typical profiles. There are three different pre-defined templates for the cruise and descent phases: fast, nominal, and slow. In contrast, there is only one pre-defined template for the ascent phase. After 
choosing the preferred concatenation of templates, the resulting sequence of segments is amended to address time constraints (RTAs) or altitude constraints during cruise. Later improvements to the TS included an increased number of templates to choose from (although each template still consists on a set of rigid constraint combinations), and the possibility of defining arbitrary portions of the flight using the GenProf language [49].

The trajectory prediction process computes the generated behaviour model in order to generate the predicted trajectory. The computation uses a pointmass aircraft model (class B), and a combination of forward and backwards integration.

\subsubsection{PHARE trajectory predictor}

The research carried out during the Programme for Harmonised ATM Research in Eurocontrol (PHARE) [30, 84] had the objective of demonstrating the feasibility of integrated ATM. As part of this project, a trajectory predictor was developed to be used both airborne - in the experimental flight management system (EFMS) - and in ground-based tools - the PHARE advanced tools.

This trajectory predictor bases the generated behaviour model in a phase table pre-defined by the user $[60,85]$. This phase table represents the most preferred flight profile for the aircraft in the absence of any constraints. A modification process adapts this phase table to meet altitude and time constraints by adding, editing, or deleting sub-phases. Sub-phases describe an operating regime, including the aircraft configuration, a thrust mode, and at most two target variables that must remain constant (i.e., altitude, speed, etc.). They also have an exit condition, defining the transitions between sub-phases. An example phase table can be found in figure 3.4.

The input to this trajectory predictor comprises the initial conditions of the aircraft, a proposed take-off time, the preferred cruise flight level, and a specification of the flight consisting of a constraint list. The constraint list is a sequence of points (named waypoints or navigation aids), each of which may have an associated constraint defining the time or altitude window that the flight is required to meet at that point.

The process of generating the behaviour model starts with the estimation of the initial sub-phase from the phase table. This is determined using a series of simple rules based on the initial aircraft state. Then, the lateral profile is built from the sequence of points in the constraint list, using circular arcs to approximate turns. With this lateral profile, combined with the initial phase table, a trajectory can be computed. However, this trajectory may not meet all the specified constraints. A series of strategies are used to address the violated constraints. These strategies are different for the climb, cruise, and descent phases. For each violated constraint, a strategy is used to address it, and a new trajectory is computed. This process is repeated iteratively until no constraints are violated, or a maximum number of iterations is reached (meaning the trajectory predictor failed to provide a solution).

The trajectory prediction process in the EFMS also used a point-mass model to compute the aircraft's trajectory from the behaviour model (class B). 


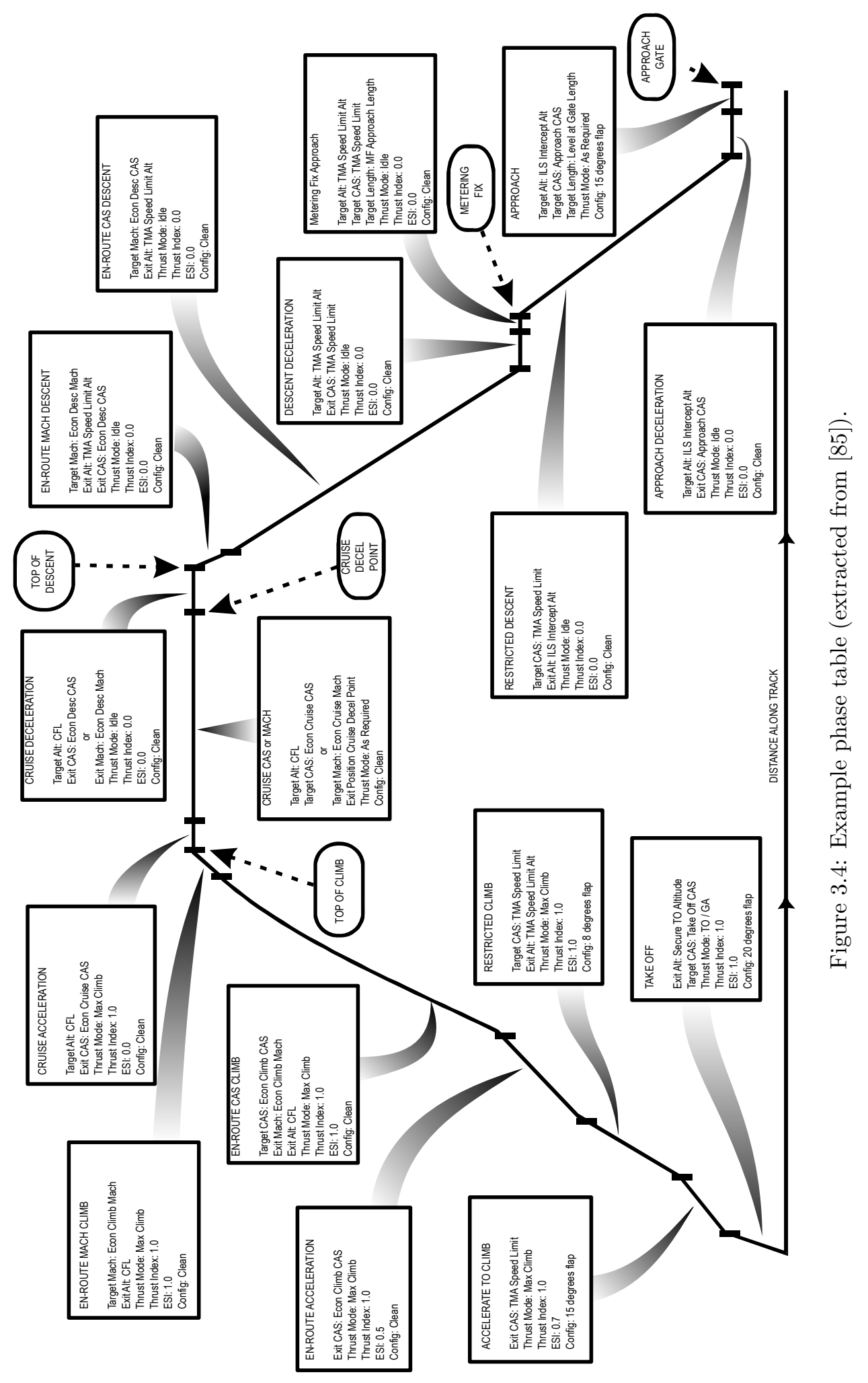




\subsubsection{Trajectory prediction in a FMS}

FMSs typically generate trajectory predictions using techniques not different from the ones described for the CTAS TS and the PHARE trajectory predictor. The details of the trajectory prediction process may vary from one FMS to another, and there is a large number of different FMSs. Moreover, this information isn't usually disclosed by the companies developing commercial FMSs. Despite the lack of detail, some general information exists in the literature [86, 87].

In FMSs, the vertical and lateral profiles are also generated separately. The lateral profile is generated from the specified route (a series of legs, airways, waypoints, etc.), but provides more detail on the transitions from one element of the route to the next. These details may be dependent on the vertical profile, e.g., turn radii depend on the ground speed, legs terminating at an altitude, etc. This dependency is usually solved by using estimated altitude and speeds. The vertical profile is then generated from a typical vertical profile that is kept in the FMS [87]. This initial profile consists on a sequence of simple manoeuvres or subphases, which are later edited to address constraints and optimize costs. The combined lateral and vertical profile are integrated using one of the integration methods discussed in section 3.2 .

\subsubsection{Other approaches to trajectory prediction}

All the trajectory predictors described in previous sections rely on a series of procedures in order to generate a predicted trajectory. That is, those methods mimic the manoeuvres performed by a pilot, and divide the flight in several flight phases. The procedures used by these trajectory predictors are known to be nearly fuel-optimal [88]. Additionally, procedurally generated trajectories can be further optimized according to some arbitrary criteria. Usually, gradient-based methods are employed for this task due to its relatively fast performance. Hybrid methods, combining gradient-based methods with other techniques (such as evolutionary algorithms or discrete pattern search), try to avoid getting stuck in a local minima [89]. However, in this case, the optimization is always constrained by the available procedures.

Other authors have followed a different approach, where different optimization techniques are used to generate the trajectory from the start. The resulting trajectory does not necessarily follow typical pilot procedures, but it is optimal according to some criterion (e.g., minimum fuel burn, flight time, emissions, etc.). These approaches don't usually fit the structure described in section 3.1. A flight path minimising the costs of a flight can be computed using dynamic programming [23], genetic algorithms [54], heuristic search algorithms [28], or a combination of both genetic algorithms and heuristic search [90]. Another way of minimising the costs of the flight consists on transforming the trajectory optimization problem into a non-linear programming problem that can be solved using various techniques. Some of these approaches use direct collocation [29, 31], shooting [37], or differential flatness [32, 36]. These non-procedural approaches generate optimized trajectories that may be difficult to read and understand by a human pilot. Moreover, the effects and consequences of flying such trajectories may be extremely difficult for a human ATCO to predict. 


\subsection{Final words}

This chapter has reviewed several trajectory predictors existing in the literature. Trajectory predictors can compute an aircraft's trajectory from a high-level (i.e., abstract) description of the trajectory. The methods used to complete the information missing in the abstract description of the trajectory vary from one trajectory predictor to another. However, procedural methods are the most usual, and the use of pre-defined profiles as a baseline for the generated trajectory is a common approach.

Trajectory predictors have many applications. Among those applications is the management of missions involving multiple aircraft. An overview of different problems related to mission management is provided in the next chapter. 



\section{Chapter 4}

\section{Management of a mission involving a fleet of UAs}

UASs are becoming less and less expensive and it is increasingly common to have fleets of UAs sharing the same airspace. In this scenario, traffic management is necessary to maintain separation between aircraft. However, the great potential of UAs lies, among other advantages, in their ability to work jointly to achieve common tasks. Therefore, the interactions between UAs aren't limited to guaranteeing safety: more complex interactions are needed to attain the objectives of a collaborative mission.

Mission management involves a number of hardware, software, and control technologies [91]. The more autonomy required, the more complex all these technologies need to be. Among the required modules of a mission management system are mission planning, trajectory generation, vehicle navigation, collision avoidance, vehicle control, fault detection, etc. This chapter focuses mainly on the mission planning problem, particularly in those scenarios where multiple UAs are involved.

Mission planning consists on processing a mission specification, which states a series of global requirements or goals, and assign tasks to each UA so that all requirements are fulfilled. In other words, mission planning transforms a mission intent into one or more flight intents. Real time re-planning may be necessary due to changes in the environment (obstacles may represent forbidden or hazardous areas, which can change through time; or, in emergency situations, even physical obstacles may collapse), changes in the fleet (some UAs could become inoperative or new UAs could be available during the mission), changes in the mission (new or cancelled requirements), or changes in available data (more precise weather forecast becomes available, new targets or obstacles are discovered, etc.). The architecture of a mission management system must take into account these circumstances, so the system can react and amend the plan so that requirements can still be fulfilled.

Tasks will usually require UAs to travel between several locations. Thus, in most cases, mission planning requires a path planner to compute - at least approximately - the paths between locations to evaluate their costs. For this reason, path planning algorithms are also reviewed in this chapter. A mission 
planner may optimize the overall performance of the fleet by computing many different combinations of assignments for each UA. Each of these assignments require the paths of the UAs to be computed. Therefore, the faster individual paths can be computed, the more assignment combinations can be assessed, and the better the resulting mission plan will be.

\subsection{Mission management system architecture}

A mission management system must deal with a myriad of different problems at different levels. A hierarchy of three levels, depicted in figure 4.1, was proposed in [91]. The highest level is responsible for the planning and coordination of the mission. The middle level computes the trajectories that individual UAs must follow to complete an assigned task, and ensures smooth state transitions based on current status of the vehicle. The lowest level is responsible for the stability of the vehicle and the management of its sensors.

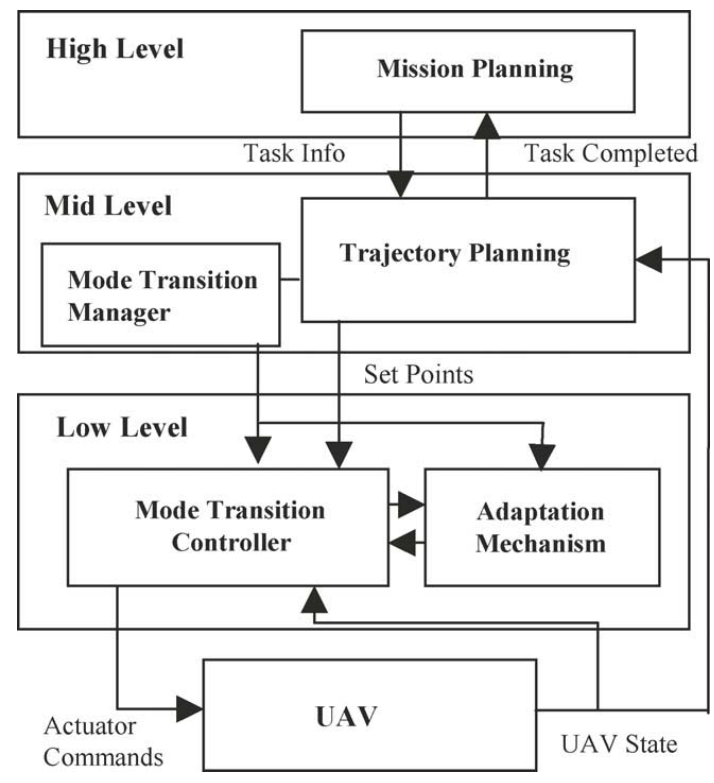

Figure 4.1: Hierarchical control architecture.

Most works focus in the middle and high levels of this hierarchy, such as the global system architecture presented in [74]. This architecture, depicted in figure 4.2, combines the previously existing AIGI and TCI with a mission engine infrastructure (MEI). The MEI comprises several elements: First, a mission intent parser processes the input and ensures that the syntax is correct. Then, the mission intent processing engine (MIPE) generates the high-level flight plans in a multi-vehicle context. In addition to the mission information, the MIPE must have access to the information regarding the assets - number and type of vehicles, capabilities (maximum speed, autonomy, communications), and payloadand the environment - including terrain mapping, static obstacles, and weather conditions. An execution supervisor monitors the ongoing mission, reacting to 


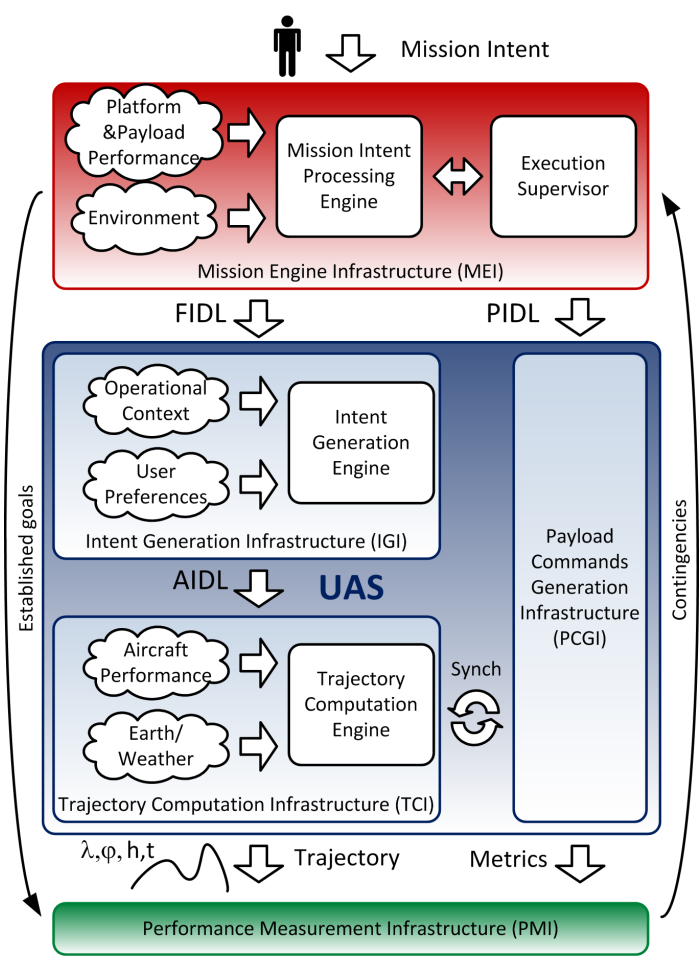

Figure 4.2: Global system architecture.

any contingencies. The type of contingencies the execution supervisor can react to depends on a series of built-in rules: it decides when a particular task must start, or whether a task under execution must be aborted. Contingencies are reported by the performance measurement infrastructure (PMI), which evaluates the results of the execution against the expected goals in the mission intent.

When designing a mission management system, the physical architecture of the system must also be taken into account. In other words, where does the processing take place. Low level modules (control, sensor data acquisition, etc.), are usually allocated inside the UAs, using a distributed model where the processing for each UA is performed on-board. On the other hand, most systems perform high level processing in a centralized ground station. Middle level processing may be either centralized [92] or distributed [74].

\subsection{Optimal distribution of tasks among UAs}

Mission planning is a computationally demanding process. It requires identifying the goals of a mission and generate a series of tasks to be performed by each individual UA. This involves searching through many possible combinations to find the best plan for the mission. Until sufficiently advanced techniques were developed and computational power evolved to make these techniques practical, mission planning was carried out by human operators [92]. However, those manual methods are slow, inefficient and fatiguing on the operator. Computer 
programs have been used to assist operators. These computerized decision aid tools have evolved to become increasingly sophisticated. Eventually, these tools lead to fully automated mission planning processes.

To solve a mission planning problem automatically, it is necessary to first express it as a mathematical problem. Different paradigms and representations have been used in the literature.

The Intelligent Systems Division at NASA's Ames Research Center developed the extensible universal remote operations planning architecture (EUROPA) $[93,94]$, a tool set for developing general-purpose planners. EUROPA is based on constraint-based temporal planning paradigm. This paradigm provides an explicit notion of time, and a constraint-based formulation of planning problems. The mission can be represented as a series of items, actions, and constraints. Items include assets (UAs), targets, paths, and any relevant mission elements. Items have a state, which can be altered through actions. Constraints represent relationships between items. The tools in EUROPA include inference and search algorithms that can be used to obtain a mission plan from the specification. Additional search algorithms (which may provide advanced heuristics for a particular application) can be also integrated.

A similar paradigm is the use of temporal action logic (TAL), which is extensively used for reasoning about actions and their effects. The threaded forwardchaining partial order planner (TFPOP) [66] admits a restricted form of TAL as input, and uses a backtracking algorithm to find a compatible plan. The output of this planner can be expressed as a series of task specification trees (TSTs), which can be executed by robotic systems.

In many cases, the mission planning problem can be considered analogous to other problems well known in the literature, such as the travelling salesman problem (TSP), the orienteeing problem [95, 96], the vehicle routing problem (VRP) [66, 97], or derivatives of these. The TSP consists of determining the shortest path that visits each target exactly once and then returns to the initial position. The orienteeing problem is a similar problem: in this case, targets have an associated score, and the objective isn't to visit all targets, but to find the subset of targets that maximizes the total score given a limited amount of resources (e.g., time or fuel). These two problems can only be used to compute the path for one vehicle, but there exist generalizations that include multiple vehicles. For example, the VRP is a generalization of the TSP for multiple vehicles. These problems are all known to be NP-hard [95] so, in general, no optimal solution can be obtained in reasonable time.

In [67], a generalization of the VRP problem is converted to a mixed integer linear programming (MILP) formulation. Other similar problems have also been formulated as a MILP [98, 99]. It has been shown that some of these formulations can be relaxed to obtain near-optimal solutions within reasonable time [98].

Genetic algorithms are another common approach to solve these problems within a reasonable time. For example, this approach is used in [97, 100] to solve the VRP and some related problems, such as the vehicle routing problem with time windows (VRPTW) or the swarm routing problem (SRP). Other approaches for similar problems are robust optimization [96], simulated annealing and auction algorithms [99]. 


\subsection{Finding paths among obstacles}

In many cases, UAs fly within a range of altitudes where the environment can be considered relatively free of static obstacles. However, when an UA moves around at low altitudes in an urban environment, it has to avoid the obstacles it may find on its way. It is of paramount importance that the paths of separate UAs are computed fast, especially when a fleet of UAs works jointly in a mission.

Finding a collision-free path in an environment with obstacles is an extensively studied problem, both in two- and three-dimensional environments.

\subsubsection{The two-dimensional case}

In a two-dimensional environment, obstacles are typically defined by polygons. It is not difficult to prove that the $2 D$ Euclidean shortest path (2ESP) between two points is a polygonal path whose inner vertices are vertices of the obstacles [101].

A 2 ESP can be found by constructing a visibility graph. A visibility graph is a data structure comprising $n$ vertices (corresponding to the vertices in the polygonal obstacles) and $m$ edges connecting every pair of vertices such that no obstacle exists between them. There are algorithms that construct the visibility graph in $O\left(n^{2}\right)$ time $^{1}[103,104]$, which was later improved to $O(m+n \log n)$ time [105]. Note there can be as many as $O\left(n^{2}\right)$ edges in the visibility graph and, in such a case, the cost of the latter algorithm is also in $O\left(n^{2}\right)$ time. Once the visibility graph is built, an algorithm such as Dijkstra's or A* can be used to find the $2 \mathrm{ESP}$ in $O(m+n \log n)$.

An alternative to constructing a visibility graph is computing the shortest path map. This map is a subdivision of the plane in regions that share the same vertex sequence in their shortest path to a particular point. An algorithm using this method can find a shortest path in $O(n \log n)$ time [106], where $n$ is the number of vertices in the obstacles.

More efficient algorithms can be found for special cases, such as the 2ESP problem inside a single polygon, which can be found in $O(n)$ time $[106,107]$. Other attempts to reduce the computational cost do so at the cost of achieving sub-optimal paths. The base line-oriented visibility line (BLOVL) algorithm [108] reduces the complexity of the problem by limiting the number of obstacles taken into account. A $\epsilon$-2ESP (a path less than $1+\epsilon$ times longer than the shortest path) can be computed in almost linear time if obstacles are convex [109]. Some other research efforts for computing near-optimal paths appear in the literature $[110,111]$, whose purpose is attaining a good enough solution with a much lower cost than computing the optimal.

\footnotetext{
${ }^{1}$ Asymptotic notation and, in particular, the big- $O$ notation will be used to express time costs of algorithms. In this notation, $O(f(n))$ represents the set of all $g(n)$ such that there exist positive constants $C$ and $n_{0}$ with $|g(n)| \leq C f(n)$ for all $n \geq n_{0}$ [102]. Thus, if the time cost of an algorithm is in $O\left(n^{2}\right)$, it means that this cost can be described using a function $g(n)$ such that $g(n) \in O\left(n^{2}\right)$.
} 


\subsubsection{The three-dimensional case}

In a three-dimensional environment, the problem is far more complex. Obstacles in this case are typically represented by polyhedra, and the $3 D$ Euclidean shortest path (3ESP) will be a polygonal path whose inner vertices lie on vertices or edges of the obstacles. This problem was shown to be NP-hard [112], and a single-exponential algorithm was later found [113]. The time cost of computing an optimal path can be reduced if some constraints are imposed on the number and shape of obstacles. A 3ESP can be computed in $O\left(n^{2} \log n\right)$ time [114] if there is only one convex polyhedron, and $O\left(n^{3} \log n\right)$ time if there are two convex polyhedra [115]. If obstacles are vertical polyhedra with $k$ distinct heights, it is possible to find a shortest path in $O\left(n^{6 k-1}\right)$ time [116].

In order to reduce the time cost, other methods avoid computing an optimal path and provide approximate $3 \mathrm{ESP}$ paths instead. Some approaches obtain a $\epsilon-3 \mathrm{ESP}$ by dividing the obstacle edges into small segments $[117,118]$, generating additional vertices. A visibility graph is constructed using these vertices in polynomial time, and the approximate shortest path is computed in that graph. Refinements of this idea have been developed later using a precision-sensitive algorithm [119, 120].

Some algorithms tackle the approximate 3ESP problem taking into account UA movement constraints. These algorithms are based on search methods $[121$, 122 ], transform the $3 \mathrm{ESP}$ problem into a 2ESP problem [123-125], or use genetic algorithms [126].

Back to the case where obstacles are vertical polyhedra, an approximate $3 \mathrm{ESP}$ path can be found in $O\left(n^{2}\right)$ time [116]. This approximate path is at most $8 \%$ longer than the shortest path if some minimum separation requirements between obstacles are satisfied.

\subsection{Final words}

An overview of some existing mission manager architectures have been provided in this chapter. Additionally, two problems related to the management of a mission involving several aircraft have been reviewed. Existing approaches to solve the mission planning problem have been included. Another problem that derives from the mission planning problem is path planning, that is, the computation of optimal paths for each individual aircraft. A review of the algorithms used for computing optimal (and near-optimal) paths has also been included.

This overview of some problems related to mission management concludes the literature review in this thesis. The next chapter introduces the languages that will be used for describing aircraft trajectories in this thesis. 


\section{Part II}

A Hierarchy of Formal
Languages 



\title{
Chapter 5
}

\section{Language hierarchy overview}

\begin{abstract}
A hierarchy of formal languages for the specification of aircraft trajectories is described in this thesis. The languages contained in this hierarchy are based on a formalization of the concept of intent, as described in chapter 2. In that chapter, two types of intent were considered: the aircraft intent and the flight intent. These can both be used to describe a trajectory, but provide a different level of detail. The aircraft intent makes it possible to provide a complete and unambiguous description of the aircraft motion, whereas the flight intent provides just the key operational requirements and constraints. However, it would be desirable to have the means to express intent information with an arbitrary level of detail. This hierarchy of languages provides these means, as it allows specifying some aspects of the aircraft motion, while leaving others unspecified.
\end{abstract}

Most of the languages composing this hierarchy have already been presented in previous publications $[16,127,128]$. These languages have been developed using a bottom-up approach. At the base of the hierarchy, the aircraft intent description language (AIDL), developed by BR\&TE, can be found. This formal language results from a rigorous mathematical analysis of the dynamic model of the aircraft. Due to its nature, the AIDL is directly related to the DoFs available to the pilot or FMS to command an aircraft. These DoFs are closed using basic guidance commands in the form of instructions. One instruction closes one DoF during a certain time interval but, by combining several instructions, a greater time interval can be covered and different DoFs can be closed. The rules that dictate how instructions are combined guarantee that all DoFs are closed, so the collection of instructions describes an aircraft trajectory unambiguously.

Different types of aircraft (e.g., fixed-wing, VTOL) have different dynamic models. As the AIDL is very closely related to these models, the AIDL designed for one type of aircraft is only valid for that particular type. Two types of aircraft are considered in this thesis: commercial fixed-wing aircraft and quadrotor aircraft.

On top of the AIDL, the intent composite description language (ICDL) provides a layer of abstraction by introducing the concept of composites. Compos- 
ites are combinations of AIDL instructions, but they allow leaving some parts of the intent unspecified: e.g., some (or even all) DoFs may be left open, and parameters of AIDL instructions may not be completely defined. A composite can be defined directly as a combination of AIDL instructions, following a syntax similar to that of the AIDL, but with some rules removed - such as the need to close all DoFs - or modified. Composites defined this way are called elementary composites. Elementary composites are usually built so that they represent a simple, understandable behaviour, such as 'maintain a level flight', 'keep a clean configuration', or 'follow a Mach/CAS descent profile'. A composite can also be defined as the aggregation of other composites. Several operations on composites are defined, which provide new semantics that are not possible in the AIDL. Therefore, any trajectory description expressed in the AIDL can be also expressed in the ICDL, whereas the opposite is not always possible. For this reason, the ICDL is an extension of the AIDL.

Following this bottom-up approach, the next language in the hierarchy is the flight intent description language (FIDL). This language is used to express flight intent information, such as flight plans, constraints or objectives (which are defined in chapter 8). Being built on top of the ICDL, the FIDL has the advantage of being able to provide descriptions of different portions of the flight with varying level of detail. For example, the climb and descent phases of the flight could be completely defined (closing all DoFs), while the cruise phase is simply defined as a list of waypoints. In the FIDL, all the semantics from the ICDL are available and some additional semantics are also provided, making the FIDL an extension of the ICDL.

The three intent-based languages outlined above can be used to describe trajectories of a single aircraft. However, regardless of how detailed these descriptions are, the actual trajectories flown by aircraft depend on external factors like the weather conditions or the aircraft performance. Thus, it is also convenient to have the means to express the trajectory itself. As discussed in section 2.1, a common way of representing trajectories is by storing a sequence of $4 \mathrm{D}$ samples. Additional state and control variables may be included in the samples. Despite not providing an intent-based description of the trajectory, sampled trajectories are also considered as part of the language hierarchy.

The definition of intent information provided in chapter 2 can be relaxed so that intent information may describe the notional path and/or constraints of one or several aircraft. This relaxation of the concept can broaden the scope of the intent-based languages. More general languages can be defined to describe trajectories of several aircraft, including interactions between such aircraft, common constraints, and global objectives. This type of description is a different type of intent, called mission intent. The mission intent description language (MIDL) can be used to describe missions involving several aircraft. This language is based on the FIDL, adding the necessary semantics to provide constraints and objectives that affect the whole mission.

Together, the AIDL, ICDL, FIDL, MIDL, and sampled trajectories can describe airborne trajectories of one or several aircraft with an arbitrary level of detail. Figure 5.1 shows the language hierarchy, where each language is represented as a box. Boxes containing other boxes represent languages extending other languages. Table 5.1 sums up some properties of the languages outlined in this chapter. 


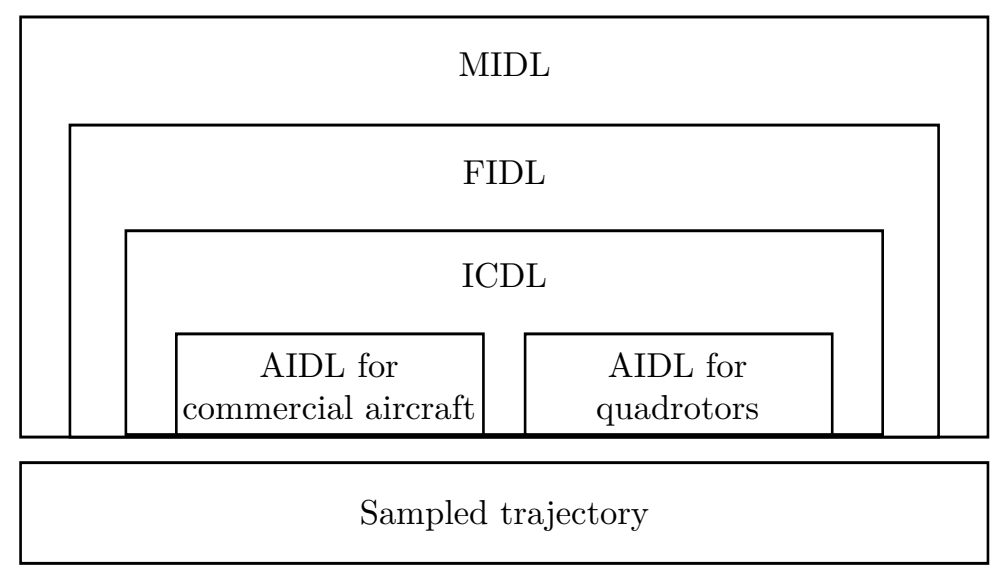

Figure 5.1: Relationships between languages in the hierarchy.

\begin{tabular}{r|c|c|c|c|c} 
& $\begin{array}{c}\text { Sampled } \\
\text { trajectory }\end{array}$ & AIDL & ICDL & FIDL & MIDL \\
\hline \hline Weather-independent & $\checkmark$ & & & & \\
Unambiguous & $\checkmark$ & $\checkmark$ & & & \\
Constraints and objectives & & & & $\checkmark$ & $\checkmark$ \\
Multiple aircraft & & & & & $\checkmark$
\end{tabular}

Table 5.1: Summary of language properties

Although the languages in the hierarchy are suitable to describe airborne aircraft trajectories, they cannot provide any description of aircraft trajectories while they are on the surface of the airport (e.g., taxiing). This is a very different scenario where the aircraft dynamics are drastically different: the movement of aircraft is constrained to a 2D surface, wind has very little effect, etc. Also, aircraft can interact with some other elements in airport surface, such as deicing platforms. The surface movement description language (SMDL) [129] can be used to describe the movement of the aircraft that takes place in airport surface before departure and after arrival. This language has not been included as part of the hierarchy, as it isn't related with any of the other languages.

The following chapters provide a more detailed definition of each of these languages. The AIDL designed for quadrotor aircraft, denoted quadrotor AIDL (QR-AIDL), is derived in the next chapter ${ }^{1}$. Then, the remaining languages of the hierarchy are described in the order they were built: chapter 7 defines the ICDL, chapter 8 defines the FIDL, and chapter 9 defines the MIDL. Finally, a complete description of the SMDL is included in chapter 10.

\footnotetext{
${ }^{1}$ The AIDL designed for fixed-wing aircraft, denoted fixed-wing AIDL (FW-AIDL), was developed prior to this thesis $[15,16,48]$. For completion, a short description of this language is included in appendix A.
} 



\section{Chapter 6}

\section{Aircraft intent description language}

In an effort to achieve consistency between disparate trajectory predictors, having an standardized method to express aircraft intent information -i.e., the input to the trajectory computation process - became a key objective [47]. This led to the development by BR\&TE of the aircraft intent description language (AIDL) $[15,16]$, a formal language derived from the generic mathematical formulation of the trajectory computation process. This makes the AIDL a rigorous and generic means of describing aircraft intent. However, it also makes the resulting definition of the AIDL highly dependant on the aircraft motion model (AMM), that is, the set of equations describing the aircraft motion. The AIDL developed in $[15,16,47]$ was designed to describe trajectories of commercial fixed-wing aircraft, and therefore it cannot be used for describing trajectories of other types of aircraft, such as vertical take-off and landing (VTOL) fixedwing aircraft, helicopters, or multirotors. For clarity, the AIDL derived from the equations of motion of a commercial fixed-wing aircraft will be hereafter referred to as the fixed-wing AIDL (FW-AIDL).

The FW-AIDL comprises a number of instructions, corresponding to guidance modes, and the rules to combine those instructions in a way such that the trajectory description is complete and unambiguous. A brief description of the FW-AIDL is included in appendix A.

The applications of formal languages to UAs, including quadrotor aircraft, are in the scope of this thesis. Since the AIDL is the foundation of the language hierarchy, it is essential to develop a variant of the AIDL that can be used to describe trajectories of quadrotor aircraft. This variant will be called the quadrotor AIDL (QR-AIDL) from now on. Some authors have designed systems that rely on the QR-AIDL to describe trajectories of quadrotor aircraft $[74,130]$. However, these works didn't address the problem of designing such variant of the AIDL, only assumed its existence.

The remainder of this chapter is devoted to developing the QR-AIDL. This development is based on the same principles that allowed the definition of the FW-AIDL. The reasoning and theory behind these principles is already covered in [16], so only practical aspects of the derivation process are included in this 
chapter.

\subsection{Derivation of the AIDL}

In order to develop the QR-AIDL, a reasonable approach is to follow the same derivation process that led to the definition of the FW-AIDL. This process is described in detail in $[15,16]$, although a summary of the process is included here for completion.

Prior to describing the derivation process, it is necessary to define two different concepts related to the degrees of freedom (DoFs) of a system:

- The number of DoFs in a mechanical system (mechanical DoF) corresponds to the number of variables required to determine its state in space. For example, a wheel on a fixed axle has one mechanical DoF (one orientation variable), and a car on a flat surface has three mechanical DoFs (two position variables and one orientation variable). In general, a rigid body in 3D space has six mechanical DoFs (three position variables and three orientation variables). A point in 3D space, however, only has three mechanical DoFs (three position variables, as the orientation of a point is irrelevant).

- The number of DoFs in a system of equations (mathematical DoFs) is defined as the order of under-determination of the system [16]. It results from subtracting the number of variables minus the number of equations of the system. If the system of equations models a dynamic system, this is also the number of control variables needed to determine the evolution of the dynamic system.

The derivation of the language starts with the assumption of the set of equations that constitute the AMM. For the FW-AIDL [15, 16], the AMM described the motion of a fixed-wing aircraft flying under several assumptions, which include symmetric and coordinated flight, and small angles of attack. Also, the aircraft is considered a point mass, so only three mechanical DoFs are studied (corresponding to a class B model, following the classification of section 3.2). The equations of the AMM form a system of ordinary differential equations (ODEs) with six mathematical $\mathrm{DoFs}^{1}$, three of which are related to the aircraft's motion, and the remaining three are related to the configuration of the aircraft (high lift devices, speed brakes, and landing gear state).

The mathematical DoFs of the AMM can be closed by providing values to the control and configuration variables. However, this is rarely useful, as doing this doesn't provide a straightforward way to model guidance modes applied in ATM and the control variables aren't usually available to the trajectory predictor. Instead, the system is closed by means of constraints. Constraints are algebraic equations of the form:

$$
g(\mathbf{X}, \mathbf{u}, t)=0
$$

\footnotetext{
${ }^{1}$ The AMM for fixed-wing aircraft has 13 variables and 7 equations, so the number of mathematical DoFs is $13-7=6$.
} 
containing state or control variables (from the state vector $\mathbf{X}$ and the control vector $\mathbf{u}$, respectively). Each constraint closes exactly one mathematical DoF of the AMM. Thus, in the FW-AIDL, six constraints are necessary to close all the mathematical DoFs.

Although the generic equation expressed in (6.1) may contain any mathematical constraint of the aircraft's motion, in practice most of the constraints used in automatic navigation can be formulated as an explicit function on one or more state or control variables. This allows the formulation of a number of generic constraints, which are included in the AIDL. Twelve of these constraints were studied in [16], which conform the building blocks of all aircraft motion expressed in FW-AIDL. These constraints can be classified in one of the following typologies:

1. Open loop input constraints represent direct action on any of the control elements. They can be expressed as:

$$
g\left(u_{i}\right)=f(t) \quad u_{i} \in \mathbf{u}
$$

2. Control law constraints model the action on one of the control elements based on a given function. They can be written as:

$$
g\left(u_{i}\right)=f(\mathbf{X}, t) \quad u_{i} \in \mathbf{u}
$$

3. Guidance law constraints represent a control objective based on one of the state variables. No control variables appear in their equations. They can be written as:

$$
g\left(X_{i}\right)=f(\mathbf{X}, t) \quad X_{i} \in \mathbf{X}
$$

4. Generalized constraints represent a control objective based on more than one of the state variables. Control variables don't appear explicitly in their equations. They can be expressed as:

$$
g(\mathbf{X})=f(\mathbf{X}, t)
$$

The AMM and the constraints form a system of DAEs, that is, a system of equations of the form:

$$
\begin{aligned}
\mathcal{F}(\dot{\mathbf{X}}, \mathbf{X}, \mathbf{u}, t) & =0 \\
\mathcal{G}(\mathbf{X}, \mathbf{u}, t) & =0
\end{aligned}
$$

where $\mathcal{F}$ and $\mathcal{G}$ are vector equations containing the differential and algebraic equations, respectively. In this case, $\mathcal{F}$ contains all the ODEs in the AMM and $\mathcal{G}$ contains the algebraic equations of the constraints. Also, $\mathbf{X}$ represents the differential variables, while $\mathbf{u}$ represents the algebraic ones. All variables in $\mathbf{X}$ and $\mathbf{u}$ are dependent on the time $t$.

By solving the initial value problem (IVP) of (6.6) for some given initial conditions, the aircraft trajectory can be obtained. For some reasonable continuity assumptions on the equations, the existence and uniqueness of a solution for a IVP problem involving ODEs has been demonstrated [131]. However, this is not applicable to DAE systems: First, the initial conditions of a DAE system must be consistent with the constraints for the system to be solvable. Second, the 
system can be non-solvable (no solution exists), weak solvable (a unique solution exists only for certain initial conditions), or strong solvable (a unique solution exists for every consistent initial conditions). Solvability must be analysed to determine which combinations of constraints lead to a strong solvable system, discarding non-solvable and weak solvable systems. The solvability analysis was carried out using Pryce's structural analysis [132] (a short description of this method can be found in [16]). This allows discriminating whether a system is solvable before solving it.

In the analysis of the AMM for the FW-AIDL, there are 12 generic constraints and 6 mathematical DoFs. Three of these constraints correspond to the three configuration DoFs, where each configuration constraint closes the corresponding DoF (i.e., one high lift, one speed breaks, and one landing gear constraints are required). That leaves 9 motion constraints and 3 motion DoFs, so the number of possible combinations ${ }^{2}$ is 84 . López-Leonés studied all the possible combinations in [16], and condensed the results in a series of rules establishing the incompatibility criteria.

Finally, the AIDL defines a number of instructions. Instructions represent specific constraints (particularized instances of the generic constraints considered in the analysis). A complete list of the constraints in the FW-AIDL can be found in table A.1 in appendix A. The AIDL also defines the execution threads, which coincides with the number of mathematical DoFs of the AMM. An aircraft intent is built by adding instructions to the threads. The incompatibility criteria resulting from the DAE solvability analysis defines the rules that establish what instruction can be added to each thread, guaranteeing that the mathematical problem has a unique solution.

Different instructions in the same thread are executed sequentially, so exactly one instruction from each execution thread is active at any given time during the flight. This ensures that the DAE system - formed by the equations in the AMM and the constraints corresponding to the active instructions- has one constraint closing each mathematical DoF. Instructions have an execution interval determining the period of time they are active (i.e., the time the corresponding constraint applies). The execution interval is defined as a condition that triggers the end of the instruction. This condition is called the end trigger of the instruction. The end of the execution interval of one instruction coincides with the start of the execution interval of the next instruction in the same thread. When the active instructions change, a new operation starts. Operations are each of the different combinations of instructions along the flight, as shown in figure 6.1. Every operation implies that a different DAE (with different constraints) must be solved. The trajectory predictor proposed in [16] computes a trajectory by sequentially solving the DAE system corresponding to each operation. This methodology is called sequential DAEs resolution (SDR). Any trajectory predictor based on any variant of the AIDL can use this methodology to compute trajectories, as most concepts (instructions, triggers, operations, threads, etc.) are common to all of them. As it will be shown in the next sections, the main differences among these variants are the number - and purpose - of threads and the types of instruction available.

\footnotetext{
${ }^{2}$ The number of possible constraint combinations without repetition is equal to $\frac{n !}{k !(n-k) !}$, where $n$ is the number of constraints and $k$ is the number of DoFs.
} 


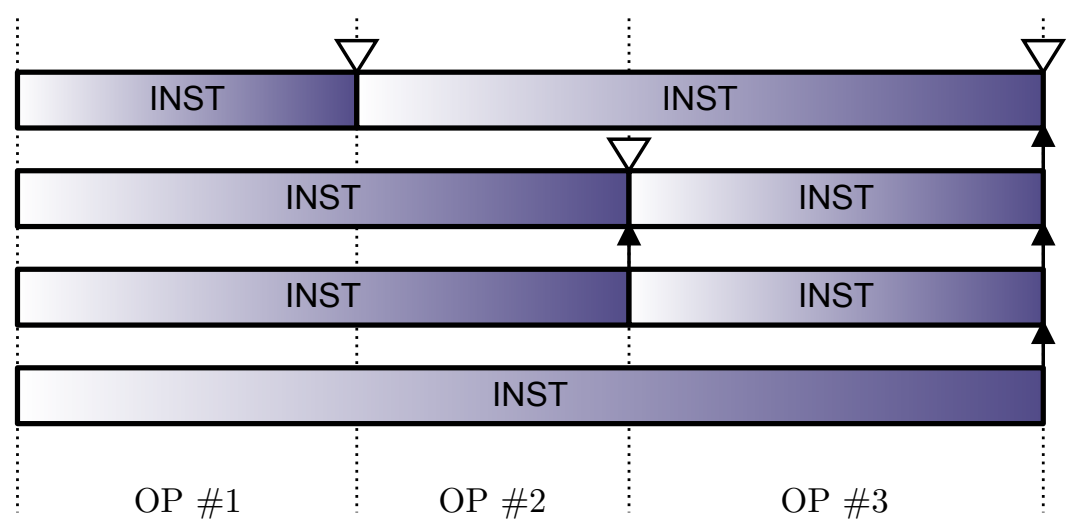

Figure 6.1: Different operations along flight in AIDL.

\subsection{Aircraft intent for quadrotors}

A process parallel to the one described in section 6.1 can be followed to develop the QR-AIDL. The first step of the process is to choose a set of equations that compose the AMM. Different sets of equations can be used, and the derivation of the language may vary depending on this choice. Therefore, the derivation process is discussed in this section using two different AMMs. The difference between these two AMMs is that the first model considers the quadrotor as a rigid body - a class A model with six mechanical DoFs-, whereas the second model considers the quadrotor as a point mass - a class B model with three mechanical DoFs.

\subsubsection{Model of six mechanical DoF}

In the literature, the motion of a quadrotor is modelled considering it a rigid body [133-142]. The first AMM used as the starting point for the QR-AIDL, which also considers the quadrotor a rigid body, is explained in appendix B. This is not the most detailed model available in the literature, but it suffices for the purposes of this thesis. This model includes the implicit ODEs in (B.28), which can also be expressed in explicit form:

$$
\begin{aligned}
\frac{\mathrm{d} \dot{x}_{e}}{\mathrm{~d} t} & =\frac{(\cos \phi \sin \theta \cos \psi+\sin \phi \sin \psi) U_{1}-k_{s}\left(\dot{x}_{e}-w_{x}\right)}{m} \\
\frac{\mathrm{d} \dot{y}_{e}}{\mathrm{~d} t} & =\frac{(\cos \phi \sin \theta \sin \psi-\sin \phi \cos \psi) U_{1}-k_{s}\left(\dot{y}_{e}-w_{y}\right)}{m} \\
\frac{\mathrm{d} \dot{z}_{e}}{\mathrm{~d} t} & =\frac{(\cos \phi \cos \theta) U_{1}-k_{u}\left(\dot{z}_{e}-w_{z}\right)}{m}-g \\
\frac{\mathrm{d} \dot{\phi}}{\mathrm{d} t} & =\frac{l U_{2}-\dot{\theta} \dot{\psi}\left(I_{z}-I_{y}\right)-J_{r} \dot{\theta} \Omega-k_{r} \dot{\phi}}{I_{x}} \\
\frac{\mathrm{d} \dot{\theta}}{\mathrm{d} t} & =\frac{l U_{3}-\dot{\phi} \dot{\psi}\left(I_{z}-I_{y}\right)-J_{r} \dot{\phi} \Omega-k_{r} \dot{\theta}}{I_{y}} \\
\frac{\mathrm{d} \dot{\psi}}{\mathrm{d} t} & =\frac{U_{4}-\dot{\phi} \dot{\theta}\left(I_{z}-I_{y}\right)-k_{r} \dot{\psi}}{I_{z}}
\end{aligned}
$$


where

$$
\begin{aligned}
U_{1} & =b\left(\Omega_{1}^{2}+\Omega_{2}^{2}+\Omega_{3}^{2}+\Omega_{4}^{2}\right) \\
U_{2} & =b\left(\Omega_{4}^{2}-\Omega_{2}^{2}\right) \\
U_{3} & =b\left(\Omega_{3}^{2}-\Omega_{1}^{2}\right) \\
U_{4} & =d\left(\Omega_{2}^{2}+\Omega_{4}^{2}-\Omega_{1}^{2}-\Omega_{3}^{2}\right) \\
\Omega & =\Omega_{2}+\Omega_{4}-\Omega_{1}-\Omega_{3}
\end{aligned}
$$

The following six trivial ODEs are also part of the AMM:

$$
\begin{array}{rlrl}
\frac{\mathrm{d} x_{e}}{\mathrm{~d} t} & =\dot{x}_{e} & & \frac{\mathrm{d} \phi}{\mathrm{d} t}=\dot{\phi} \\
\frac{\mathrm{d} y_{e}}{\mathrm{~d} t}=\dot{y}_{e} & \frac{\mathrm{d} \theta}{\mathrm{d} t}=\dot{\theta} \\
\frac{\mathrm{d} z_{e}}{\mathrm{~d} t}=\dot{z}_{e} & \frac{\mathrm{d} \psi}{\mathrm{d} t}=\dot{\psi}
\end{array}
$$

Descriptions of the parameters and variables in the equations above can be found in appendix B, and a brief description is also provided in table 6.1.

\begin{tabular}{c|l} 
Variable & Description \\
\hline \hline$x_{e}, y_{e}, z_{e}$ & $\begin{array}{l}\text { Quadrotor's position with respect to an earth-fixed frame } \\
\text { in cartesian coordinates. } \\
\text { Quadrotor's speed with respect to the same earth-fixed } \\
\dot{x}_{e}, \dot{y}_{e}, \dot{z}_{e}\end{array}$ \\
$\phi, \theta, \psi$ & $\begin{array}{l}\text { frame. } \\
\text { Quadrotor's rotation around axes } x_{e}, y_{e}, \text { and } z_{e} . \text { These } \\
\text { angles are also known as roll, pitch, and yaw, respectively. }\end{array}$ \\
$\dot{\phi}, \dot{\theta}, \dot{q u a d r o t o r ' s ~ a n g u l a r ~ s p e e d ~ w i t h ~ r e s p e c t ~ t o ~ t h e ~ s a m e ~ a x e s . ~}$ \\
$m$ & $\begin{array}{l}\text { Mass of the quadrotor. } \\
\text { Thrust coefficient. }\end{array}$ \\
$g$ & Acceleration due to gravity. \\
$l$ & Distance between propellers and center of mass. \\
$d$ & Drag coefficient. \\
$J_{r}$ & Rotor inertia. \\
$k_{s}, k_{u}$ & Horizontal and vertical friction coefficients. \\
$k_{r}$ & Friction coefficient due to rotational motion. \\
$w_{x}, w_{y}, w_{z}$ & Wind speed along axes $x_{e}, y_{e}$, and $z_{e}$. \\
$I_{x}, I_{y}, I_{z}$ & Moments of inertia of the quadrotor. \\
$\Omega_{1}, \Omega_{2}, \Omega_{3}, \Omega_{4}$ & Rotation speed of propellers.
\end{tabular}

Table 6.1: Description of variables in quadrotor AMM.

The system of equations comprising the AMM has:

- One independent variable: $t$.

- Sixteen dependent variables: $x_{e}, y_{e}, z_{e}, \phi, \theta, \psi, \dot{x}_{e}, \dot{y}_{e}, \dot{z}_{e}, \dot{\phi}, \dot{\theta}, \dot{\psi}, \Omega_{1}, \Omega_{2}$, $\Omega_{3}$, and $\Omega_{4}$.

- Four control variables: $\Omega_{1}, \Omega_{2}, \Omega_{3}$, and $\Omega_{4}$. 
- Twelve state variables: $x_{e}, y_{e}, z_{e}, \phi, \theta, \psi, \dot{x}_{e}, \dot{y}_{e}, \dot{z}_{e}, \dot{\phi}, \dot{\theta}$, and $\dot{\psi}$.

- Twelve equations ${ }^{3}$.

With this information, it can be concluded that the AMM has $16-12=4$ mathematical DoFs. This implies that the resulting QR-AIDL will have four separate execution threads, instead of the six threads in the FW-AIDL. This difference can be attributed to two facts: First, no configuration parameters are considered in quadrotor aircraft, so no configuration threads are necessary. And second, while commercial fixed-wing aircraft are assumed to always move forward (as the flight is assumed to be symmetric and coordinated), quadrotor aircraft's lateral movement is independent of the direction it's facing. Therefore, the four mathematical DoFs are all related to the aircraft's motion.

This system of equations can be solved if the values for the control variables $\Omega_{i}$ are provided. However, in most cases, it will be desirable to define the quadrotor's behaviour using four algebraic constraints of the form:

$$
g(\mathbf{X}, \mathbf{u}, t)
$$

where $\mathbf{X}$ is the state vector, containing the state variables (which are also the differential variables), and $\mathbf{u}$ is the control vector, containing the control variables (which are also the algebraic variables).

\section{Generic constraints}

The next step in the process is to set the generic constraints that will be used to define the quadrotor's motion. These constraints have to match the typologies described in section 6.1. The choice of generic constraints used here includes one open loop input and one control law constraint for each control variable, one guidance law constraint for most state variables, and two generalized constraints. All generic constraints are listed in table 6.2.

Most of the proposed generic constraints are self explanatory. Open loop input, control law, and guidance law constraints can be used to define the evolution of one control or state variable. The generalized constraints are more complex, but they are particularly interesting for the specification of quadrotor trajectories as they allow defining the horizontal speed in polar coordinates.

Horizontal speed (or ground speed) could be specified in cartesian coordinates using guidance law constraints for both $\dot{x}_{e}$ and $\dot{y}_{e}$. However, it is more intuitive and convenient to express them in polar coordinates, providing the module (a scalar value) and the direction (an angle) of the horizontal speed.

The module of the horizontal speed can be specified using generalized constraints of the form $g\left(\dot{x}_{e}, \dot{y}_{e}\right)=f(\mathbf{X}, t)$. A first approach would be to explicitly include the module in the constraint, as shown in (6.15).

$$
\sqrt{\dot{x}_{e}^{2}+\dot{y}_{e}^{2}}=f(\mathbf{X}, t)
$$

However, this approach would not work well when the horizontal speed is close to zero. This is not a problem for fixed-wing aircraft but, in this case, it

\footnotetext{
${ }^{3}$ Equations (6.8)-(6.12) contain definitions of some intermediate variables and are not included in the count.
} 


\begin{tabular}{l|l|l} 
Typology & $\begin{array}{l}\text { Generic } \\
\text { constraints }\end{array}$ & $\begin{array}{l}\text { Representative } \\
\text { constraints }\end{array}$ \\
\hline \hline \multirow{5}{*}{ Open loop input } & $g\left(\Omega_{1}\right)=f(t)$ & $g\left(\Omega_{1}\right)=0$ \\
& $g\left(\Omega_{2}\right)=f(t)$ & $g\left(\Omega_{2}\right)=0$ \\
& $g\left(\Omega_{3}\right)=f(t)$ & $g\left(\Omega_{3}\right)=0$ \\
& $g\left(\Omega_{4}\right)=f(t)$ & $g\left(\Omega_{4}\right)=0$ \\
\hline \multirow{5}{*}{ Control law } & $g\left(\Omega_{1}\right)=f(\mathbf{X}, t)$ & $g\left(\Omega_{1}\right)=0$ \\
& $g\left(\Omega_{2}\right)=f(\mathbf{X}, t)$ & $g\left(\Omega_{2}\right)=0$ \\
& $g\left(\Omega_{3}\right)=f(\mathbf{X}, t)$ & $g\left(\Omega_{3}\right)=0$ \\
& $g\left(\Omega_{4}\right)=f(\mathbf{X}, t)$ & $g\left(\Omega_{4}\right)=0$ \\
\hline \multirow{5}{*}{ Guidance law } & $g\left(z_{e}\right)=f(\mathbf{X}, t)$ & $g\left(z_{e}\right)=0$ \\
& $g(\phi)=f(\mathbf{X}, t)$ & $g(\phi)=0$ \\
& $g(\theta)=f(\mathbf{X}, t)$ & $g(\theta)=0$ \\
& $g(\psi)=f(\mathbf{X}, t)$ & $g(\psi)=0$ \\
& $g\left(\dot{z}_{e}\right)=f(\mathbf{X}, t)$ & $g\left(\dot{z}_{e}\right)=0$ \\
& $g(\dot{\phi})=f(\mathbf{X}, t)$ & $g(\dot{\phi})=0$ \\
& $g(\dot{\theta})=f(\mathbf{X}, t)$ & $g(\dot{\theta})=0$ \\
& $g(\dot{\psi})=f(\mathbf{X}, t)$ & $g(\dot{\psi})=0$ \\
\hline \multirow{2}{*}{ Generalized constraints } & $g\left(\dot{x}_{e}, \dot{y}_{e}\right)=f(\mathbf{X}, t)$ & $g\left(\dot{x}_{e}, \dot{y}_{e}\right)=0$ \\
& $g\left(\dot{x}_{e}, \dot{y}_{e}, f(\mathbf{X}, t)\right)=0$ & $g\left(\dot{x}_{e}, \dot{y}_{e}\right)=0$
\end{tabular}

Table 6.2: Generic and representative constraints for solvability analysis.

must be taken into account. Alternatively, this generalized constraint can be expressed as

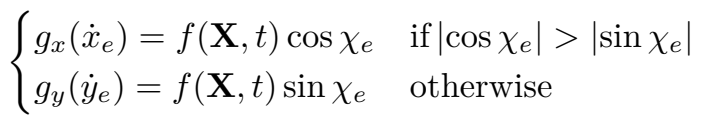

where $\chi_{e}$ is the direction of movement of the quadrotor (with respect to an Earth-fixed reference frame), and $g_{x}$ and $g_{y}$ are linear functions. This alternative expression defines only the module of the horizontal speed along one of the axes $\left(x_{e}\right.$ or $\left.y_{e}\right)$. The module along the other axis is implicitly defined by the direction of the horizontal speed.

In order to complete the definition of the horizontal speed, its direction must be specified. Again, a first approach would be to explicitly include the direction in the constraint, as in the equation below:

$$
\arctan \frac{\dot{y}_{e}}{\dot{x}_{e}}=f(\mathbf{X}, t)
$$

This equation has obvious problems when $\dot{x}_{e}$ or the horizontal speed are near zero. An alternate equation for expressing the constraint in (6.17) is the following:

$$
g_{y}\left(\dot{y}_{e}\right) \cos f(\mathbf{X}, t)-g_{x}\left(\dot{x}_{e}\right) \sin f(\mathbf{X}, t)=0
$$

where $g_{x}$ and $g_{y}$ are linear functions. In this case, the generalized constraint is of the form $g\left(\dot{x}_{e}, \dot{y}_{e}, f(\mathbf{X}, t)\right)=0$.

When the two generalized constraints are used together, they completely define the horizontal speed. The first one sets the value of the greatest (in absolute 
value) cartesian component of the horizontal speed ${ }^{4}$. The other component will be implicitly defined, as the two cartesian components are bound by the second generalized constraint.

Conversely, the direction and module of the horizontal speed can be obtained from its cartesian coordinates using equations (6.19) and (6.20), given that the horizontal speed is not zero.

$$
\begin{aligned}
v_{g} & =\sqrt{\dot{x}_{e}^{2}+\dot{y}_{e}^{2}} \\
\chi_{e} & =\operatorname{atan} 2\left(\dot{y}_{e}, \dot{x}_{e}\right)
\end{aligned}
$$

The FW-AIDL also included a generalized constraint defining the horizontal profile geometrically. While the way this constraint was defined was very useful in that case, in the case of quadrotors it is not a viable option. Generalized constraints of the form $g\left(x_{e}, y_{e}\right)=0$ define a path in which the quadrotor must move, but it doesn't define the direction of movement. This is not a problem for fixed-wing aircraft, as their horizontal speed is never zero, and the direction of the horizontal speed in the initial conditions disambiguates the direction of motion. However, in quadrotor aircraft, the direction of the horizontal speed in the initial conditions may be undefined if its module is zero, i.e., the quadrotor is hovering without moving, leading to an ambiguous definition of the flight. For this reason, generalized constraints defining the horizontal profile geometrically are not considered in the QR-AIDL.

With a set of generic constraints defined, the next step is to determine whether the DAE problem is solvable or not for each possible combination of constraints. In this solvability analysis, as in [16], only the dependent part of the equation is considered relevant (this is, in most cases, the left hand side of the equation), as they are the minimum expression keeping the purpose of the constraint. Therefore, the representative constraints in table 6.2 are used instead of the generic constraints.

\section{Solvability analysis}

In total, 14 different representative constraints ${ }^{5}$ must be taken into account in the solvability analysis. A complete analysis would require any possible combination of constraints to be studied. In this case, there are 1001 possible combinations $^{6}$, and a thorough analysis is completely out of the scope of this thesis. However, the analysis of only some of these combinations is sufficient to state that the approach with six mechanical DoFs is not suitable for the definition of the QR-AIDL, as it is discussed below.

As it is shown in appendix A, the FW-AIDL has three DoFs related to the aircraft's motion: two of them related to the vertical profile, and one of them related to the horizontal profile. In the QR-AIDL, it would be desirable to have a similar distribution, with an additional DoF for the orientation $(\psi)$ of the quadrotor. For example, the following combination of four generic constraints

\footnotetext{
${ }^{4}$ Since $\left|\cos \chi_{e}\right| \geq\left|\sin \chi_{e}\right|$ implies $\left|\dot{x}_{e}\right| \geq\left|\dot{y}_{e}\right|$.

${ }^{5}$ There are four open loop input / control law constraints (note that the representative constraints for those two typologies are identical), eight guidance law constraints, and two generalized constraints.

${ }^{6}$ Using the same formula as before, there are $\frac{14 !}{4 !(14-4) !}=1001$ combinations.
} 
could be used to specify the quadrotor's behaviour. The first two constraints define the vertical profile (by defining the horizontal and vertical speeds), the third constraint define the lateral profile (by defining the direction of the horizontal speed), and a fourth constraint defines where the quadrotor must be pointing at:

$$
\begin{aligned}
g_{1}\left(\dot{z}_{e}\right) & =f_{1}(\mathbf{X}, t) \\
g_{2}\left(\dot{x}_{e}, \dot{y}_{e}\right) & =f_{2}(\mathbf{X}, t) \\
g_{3}\left(\dot{x}_{e}, \dot{y}_{e}, f_{3}(\mathbf{X}, t)\right) & =0 \\
g_{4}(\psi) & =f_{4}(\mathbf{X}, t)
\end{aligned}
$$

Using the corresponding representative constraints, the equations to be considered in the analysis would be:

$$
\begin{aligned}
g_{1}\left(\dot{z}_{e}\right) & =0 \\
g_{2}\left(\dot{x}_{e}, \dot{y}_{e}\right) & =0 \\
g_{3}\left(\dot{x}_{e}, \dot{y}_{e}\right) & =0 \\
g_{4}(\psi) & =0
\end{aligned}
$$

The solvability of this DAE system, comprising equations in (6.7) and (6.22), can be determined using Pryce's structural analysis. This method of analyisis includes several steps [132] that lead to the formation of the system Jacobian matrix $\mathbf{J}$ :

$$
\begin{aligned}
& \mathbf{J}= \\
& {\left[\begin{array}{cccccccccc}
m & 0 & 0 & 0 & 0 & 0 & 2 \Omega_{1} b R_{3,1} & 2 \Omega_{2} b R_{3,1} & 2 \Omega_{3} b R_{3,1} & 2 \Omega_{4} b R_{3,1} \\
0 & m & 0 & 0 & 0 & 0 & 2 \Omega_{1} b R_{3,2} & 2 \Omega_{2} b R_{3,2} & 2 \Omega_{3} b R_{3,2} & 2 \Omega_{4} b R_{3,2} \\
0 & 0 & m & 0 & 0 & 0 & 2 \Omega_{1} b R_{3,3} & 2 \Omega_{2} b R_{3,3} & 2 \Omega_{3} b R_{3,3} & 2 \Omega_{4} b R_{3,3} \\
0 & 0 & 0 & I_{x} & 0 & 0 & 0 & 2 \Omega_{2} b l & 0 & 2 \Omega_{4} b l \\
0 & 0 & 0 & 0 & I_{y} & 0 & 2 \Omega_{1} b l & 0 & 2 \Omega_{3} b l & 0 \\
0 & 0 & 0 & 0 & 0 & I_{z} & -1 & 1 & -1 & 1 \\
0 & 0 & \frac{\mathrm{d} g_{1}}{\mathrm{~d} \dot{z}_{e}} & 0 & 0 & 0 & 0 & 0 & 0 & 0 \\
\frac{\mathrm{d} g_{2}}{\mathrm{~d} \dot{x}_{e}} & \frac{\mathrm{d} g_{2} \dot{y}_{e}}{0} & 0 & 0 & 0 & 0 & 0 & 0 & 0 & 0 \\
\frac{\mathrm{d} g_{3}}{\mathrm{~d} \dot{x}_{e}} & \frac{\mathrm{d} g_{3}}{\mathrm{~d} \dot{y}_{e}} & 0 & 0 & 0 & 0 & 0 & 0 & 0 & 0 \\
0 & 0 & 0 & 0 & 0 & \frac{\mathrm{d} g_{4}}{\mathrm{~d} \psi} & 0 & 0 & 0 & 0
\end{array}\right]}
\end{aligned}
$$

where $R_{3,1}, R_{3,2}$, and $R_{3,3}$ are elements of the rotation matrix $\mathbf{R}$, as defined in equation (B.4):

$$
\begin{aligned}
& R_{3,1}=\cos \phi \sin \theta \cos \psi+\sin \phi \sin \psi \\
& R_{3,2}=\cos \phi \sin \theta \sin \psi-\sin \phi \cos \psi \\
& R_{3,3}=\cos \phi \cos \theta
\end{aligned}
$$

Pryce's structural analysis states that, given some consistent initial conditions composed of a state vector $\mathbf{X}_{0}$ and a time $t_{0}$, the condition for the solvability of the system is that $\mathbf{J}$ particularized in $\mathbf{X}_{0}$ and $t_{0}$ is non-singular. In this case, however, $\mathbf{J}$ is a singular matrix regardless of the initial conditions, and the DAE system is non-solvable. 
The same result is obtained when analysing other combinations of constraints that represent useful guidance modes for a quadrotor. The system Jacobian matrix is singular in most of the analysed cases. The only exceptions occur when most of the constraints are related to control variables (open loop input or control law). Of course, when the constraints of the system are the four open loop input constraints, the system behaves as an ODE system and always provides a solution regardless of the initial conditions.

Additionally, a software prototype of a trajectory predictor using these equations was developed. While it could predict trajectories for some scenarios, the computation of the trajectory failed in most cases, as the numerical DAE system solver couldn't find solutions. The failing cases match the combinations of constraints deemed non-solvable by Pryce's structural analysis. These problems led to discard the six DoFs model as the starting point for the QR-AIDL. In the next section, a simplified three DoFs model is used, which will lead to a successful development of the QR-AIDL.

\subsubsection{Model of three mechanical DoF}

Considering the quadrotor as a rigid body leads to more precise models. This is a very important factor in control system design, an area that has traditionally motivated the development of more and more accurate models. However, requirements for trajectory predictors are not as demanding. A simplified AMM with three mechanical DoFs (class B) is used in this section to derive the QRAIDL. In this AMM, the quadrotor is treated as a point mass where its rotation is a control input. The differences in the speed of the different propellers becomes irrelevant, and a single value, $\Omega_{T}^{2}$, is used to represent the average square thrust. The average square thrust is related to the speed of each propeller by the following equation:

$$
\Omega_{T}^{2}=\frac{1}{4}\left(\Omega_{1}^{2}+\Omega_{2}^{2}+\Omega_{3}^{2}+\Omega_{4}^{2}\right)
$$

The system of differential equations in (6.7) and (6.13) is used as the starting point for the new AMM. The ODEs related to the torque and rotation of the quadrotor are removed from the system. The rotation in this model is no longer a result of a series of forces acting on the quadrotor, but a control input that can be provided directly to the system. The average square thrust is also part of the control input, replacing variables $\Omega_{1}, \Omega_{2}, \Omega_{3}$ and $\Omega_{4}$ from the model of six mechanical DoFs. The resulting ODE system can be written as:

$$
\begin{aligned}
\frac{\mathrm{d} \dot{x}_{e}}{\mathrm{~d} t} & =\frac{(\cos \phi \sin \theta \cos \psi+\sin \phi \sin \psi) U_{1}-k_{s}\left(\dot{x}_{e}-w_{x}\right)}{m} \\
\frac{\mathrm{d} \dot{y}_{e}}{\mathrm{~d} t} & =\frac{(\cos \phi \sin \theta \sin \psi-\sin \phi \cos \psi) U_{1}-k_{s}\left(\dot{y}_{e}-w_{y}\right)}{m} \\
\frac{\mathrm{d} \dot{z}_{e}}{\mathrm{~d} t} & =\frac{(\cos \phi \cos \theta) U_{1}-k_{u}\left(\dot{z}_{e}-w_{z}\right)}{m}-g
\end{aligned}
$$

where

$$
U_{1}=4 b \Omega_{T}^{2}
$$


Plus the following three trivial ODEs, which are also part of the AMM:

$$
\begin{aligned}
\frac{\mathrm{d} x_{e}}{\mathrm{~d} t} & =\dot{x}_{e} \\
\frac{\mathrm{d} y_{e}}{\mathrm{~d} t} & =\dot{y}_{e} \\
\frac{\mathrm{d} z_{e}}{\mathrm{~d} t} & =\dot{z}_{e}
\end{aligned}
$$

This simplified system of equations has:

- One independent variable: $t$.

- Ten dependent variables: $x_{e}, y_{e}, z_{e}, \dot{x}_{e}, \dot{y}_{e}, \dot{z}_{e}, \phi, \theta, \psi$, and $\Omega_{T}$.

- Four control variables: $\phi, \theta, \psi$, and $\Omega_{T}$.

- Six state variables: $x_{e}, y_{e}, z_{e}, \dot{x}_{e}, \dot{y}_{e}$, and $\dot{z}_{e}$

- Six equations.

The number of mathematical DoFs of this system is also 4 (10 dependent variables minus 6 equations), so the number of constraints needed to close the system (and therefore the number of separate execution threads in the QRAIDL) is the same as that of the AMM of six mechanical DoFs. As in that case, the four mathematical DoFs are all related to the aircraft's motion.

Variables $\dot{x}_{e}, \dot{y}_{e}$, and $\dot{z}_{e}$ represent the quadrotor's speed with respect to an Earth-fixed reference frame E. However, in many cases it will be useful to work with the quadrotor's speed with respect to the mass of air. A bodyfixed Earth-normal reference frame $A$, with the origin of coordinates fixed at the quadrotor's center of mass (thus body-fixed) and the same orientation as $E$ (thus Earth-normal), can be used to compute these speeds, with:

$$
\begin{aligned}
\dot{x}_{a} & =\dot{x}_{e}-w_{x} \\
\dot{y}_{a} & =\dot{y}_{e}-w_{y} \\
\dot{z}_{a} & =\dot{z}_{e}-w_{z}
\end{aligned}
$$

where $w_{x}, w_{y}$, and $w_{z}$ are the local wind speeds along the axes of the reference frame $A$.

\section{Generic constraints}

The generic constraints used to describe meaningful quadrotor behaviour are mostly the same used in the previous section. Only a few changes are needed to reflect the changes in the control and state variables. Therefore, there are still open loop input and control law constraints for each control variable, guidance law constraints for most state variables, and the same two generalized constraints. All generic constraints are listed in table 6.3.

Again, the representative constraints are obtained by taking only the dependent part of the equation (which is the left hand side of the equation in most cases). Solvability of the DAE system will be analysed using the representative constraints in table 6.3 . 


\begin{tabular}{l|l|l} 
Typology & $\begin{array}{l}\text { Generic } \\
\text { constraint }\end{array}$ & $\begin{array}{l}\text { Representative } \\
\text { constraint }\end{array}$ \\
\hline \hline \multirow{5}{*}{ Open loop input } & $g(\phi)=f(t)$ & $g(\phi)=0$ \\
& $g(\theta)=f(t)$ & $g(\theta)=0$ \\
& $g(\psi)=f(t)$ & $g(\psi)=0$ \\
& $g\left(\Omega_{T}\right)=f(t)$ & $g\left(\Omega_{T}\right)=0$ \\
\hline \multirow{3}{*}{ Control law } & $g(\phi)=f(\mathbf{X}, t)$ & $g(\phi)=0$ \\
& $g(\theta)=f(\mathbf{X}, t)$ & $g(\psi)=0$ \\
& $g(\psi)=f(\mathbf{X}, t)$ & $g\left(\Omega_{T}\right)=0$ \\
\hline \multirow{2}{*}{ Guidance law } & $g\left(\Omega_{T}\right)=f(\mathbf{X}, t)$ & $g\left(z_{e}\right)=0$ \\
& $g\left(z_{e}\right)=f(\mathbf{X}, t)$ & $g\left(\dot{z}_{e}\right)=0$ \\
\hline \multirow{2}{*}{ Generalized constraints } & $g\left(\dot{z}_{e}\right)=f(\mathbf{X}, t)$ & $g\left(\dot{x}_{e}, \dot{y}_{e}\right)=f(\mathbf{X}, t)$ \\
& $g\left(\dot{x}_{e}, \dot{y}_{e}, f(\mathbf{X}, t)\right)=0$ & $g\left(\dot{x}_{e}, \dot{y}_{e}\right)=0$ \\
&
\end{tabular}

Table 6.3: New representative constraints for solvability analysis.

\section{Solvability analysis}

In this case, the number of generic constraints has been substantially reduced with respect to the model of six mechanical DoFs. There are 70 combinations $^{7}$ of representative constraints to be analysed, which is a much more attainable task. However, a thorough analysis including all combinations is also out of the scope of this thesis. Instead, this section will focus on some of the most common control/guidance modes.

Although originally intended for the 6-DoFs model, the set of generic constraints in (6.21) is a desirable combination for describing the quadrotor's behaviour also for the 3-DoFs model. The solvability of the corresponding set of representative constraints in (6.22) can be repeated with the new AMM using the same method. In this case, the resulting system Jacobian matrix $\mathbf{J}$ is:

$$
\mathbf{J}=\left[\begin{array}{ccccccc}
m & 0 & 0 & 4 b \Omega_{T}^{2} \frac{\mathrm{d} R_{3,1}}{\mathrm{~d} \phi} & 4 b \Omega_{T}^{2} \frac{\mathrm{d} R_{3,1}}{\mathrm{~d} \theta} & 4 b \Omega_{T}^{2} \frac{\mathrm{d} R_{3,1}}{\mathrm{~d} \psi} & 8 b \Omega_{T} R_{3,1} \\
0 & m & 0 & 4 b \Omega_{T}^{2} \frac{\mathrm{d} R_{3,2}}{\mathrm{~d} \phi} & 4 b \Omega_{T}^{2} \frac{\mathrm{d} R_{3,2}}{\mathrm{~d} \theta} & 4 b \Omega_{T}^{2} \frac{\mathrm{d} R_{3,2}}{\mathrm{~d} \psi} & 8 b \Omega_{T} R_{3,2} \\
0 & 0 & m & 4 b \Omega_{T}^{2} \frac{\mathrm{d} R_{3,3}}{\mathrm{~d} \phi} & 4 b \Omega_{T}^{2} \frac{\mathrm{d} R_{3,3}}{\mathrm{~d} \theta} & 0 & 8 b \Omega_{T} R_{3,3} \\
0 & 0 & \frac{\mathrm{d} g_{1}}{\mathrm{~d} \dot{z}_{e}} & 0 & 0 & 0 & 0 \\
\frac{\mathrm{d} g_{2}}{\mathrm{~d} \dot{x}_{e}} & \frac{\mathrm{d} g_{2}}{\mathrm{~d} \dot{y}_{e}} & 0 & 0 & 0 & 0 & 0 \\
\frac{\mathrm{d} g_{3}}{\mathrm{~d} \dot{x}_{e}} & \frac{\mathrm{d} g_{3}}{\mathrm{~d} \dot{y}_{e}} & 0 & 0 & 0 & 0 & 0 \\
0 & 0 & 0 & 0 & 0 & \frac{\mathrm{d} g_{4}}{\mathrm{~d} \psi} & 0
\end{array}\right]
$$

where

$$
\begin{aligned}
\frac{\mathrm{d} R_{3,1}}{\mathrm{~d} \phi} & =-\sin \phi \sin \theta \cos \psi+\cos \phi \sin \psi \\
\frac{\mathrm{d} R_{3,1}}{\mathrm{~d} \theta} & =\cos \phi \cos \theta \cos \psi
\end{aligned}
$$

\footnotetext{
${ }^{7}$ There are eight different representative constraints and, therefore, $\frac{8 !}{4 !(8-4) !}=70$ combinations of such constraints.
} 


$$
\begin{aligned}
\frac{\mathrm{d} R_{3,1}}{\mathrm{~d} \psi} & =-\cos \phi \sin \theta \sin \psi+\sin \phi \cos \psi \\
\frac{\mathrm{d} R_{3,2}}{\mathrm{~d} \phi} & =-\sin \phi \sin \theta \sin \psi-\cos \phi \cos \psi \\
\frac{\mathrm{d} R_{3,2}}{\mathrm{~d} \theta} & =\cos \phi \cos \theta \sin \psi \\
\frac{\mathrm{d} R_{3,2}}{\mathrm{~d} \psi} & =\cos \phi \sin \theta \cos \psi+\sin \phi \sin \psi \\
\frac{\mathrm{d} R_{3,3}}{\mathrm{~d} \phi} & =-\sin \phi \cos \theta \\
\frac{\mathrm{d} R_{3,3}}{\mathrm{~d} \theta} & =-\cos \phi \sin \theta
\end{aligned}
$$

As in the previous analysis, it must be ensured that for the initial conditions comprised of a state vector $\mathbf{X}_{0}$ and a time $t_{0}, \mathbf{J}$ is non-singular. Equivalently, it must be ensured that the determinant of $\mathbf{J}$ is not zero. In this case, the determinant of $\mathbf{J}$ is:

$$
\operatorname{det} \mathbf{J}=-128 \Omega_{T}^{3} b^{3} \cos \phi\left(\frac{\mathrm{d} g_{3}}{\mathrm{~d} \dot{x}_{e}} \frac{\mathrm{d} g_{2}}{\mathrm{~d} \dot{y}_{e}}-\frac{\mathrm{d} g_{3}}{\mathrm{~d} \dot{y}_{e}} \frac{\mathrm{d} g_{2}}{\mathrm{~d} \dot{x}_{e}}\right) \frac{\mathrm{d} g_{1}}{\mathrm{~d} \dot{z}_{e}} \frac{\mathrm{d} g_{4}}{\mathrm{~d} \psi}
$$

It can be safely assumed that $b$, a constant provided by the APM of the quadrotor, is non-zero ${ }^{8}$. It must also be assumed that the average square thrust is non-zero, that is, the propellers are spinning with speed greater than zero. Another requirement is that $\cos \phi \neq 0$. We will assume from now on that the quadrotor doesn't tilt more than 90 degrees from upwards position or, in other words, that $\cos \phi \cos \theta>0$. This ensures that the previous requirement is always met.

Finally, some requirements on the constraints must be met to make $\mathbf{J}$ nonsingular. Requirements on constraints $g_{1}$ and $g_{4}$, i.e., $\frac{\mathrm{d} g_{1}}{\mathrm{~d} \dot{z}_{e}} \neq 0$ and $\frac{\mathrm{d} g_{4}}{\mathrm{~d} \psi} \neq 0$, are easy to meet, and constraints that don't meet these requirements are not of interest in a trajectory predictor. The last requirement is a bit trickier:

$$
\frac{\mathrm{d} g_{3}}{\mathrm{~d} \dot{x}_{e}} \frac{\mathrm{d} g_{2}}{\mathrm{~d} \dot{y}_{e}}-\frac{\mathrm{d} g_{3}}{\mathrm{~d} \dot{y}_{e}} \frac{\mathrm{d} g_{2}}{\mathrm{~d} \dot{x}_{e}} \neq 0
$$

This is equivalent to requiring matrix

$$
\left[\begin{array}{ll}
\frac{\mathrm{d} g_{2}}{\mathrm{~d} \dot{x}_{e}} & \frac{\mathrm{d} g_{2}}{\mathrm{~d} \dot{y}_{e}} \\
\frac{\mathrm{d} g_{3}}{\mathrm{~d} \dot{x}_{e}} & \frac{\mathrm{d} g_{3}}{\mathrm{~d} \dot{y}_{e}}
\end{array}\right]
$$

to be non-singular, or requiring vectors $\left(\frac{\mathrm{d} g_{2}}{\mathrm{~d} \dot{x}_{e}}, \frac{\mathrm{d} g_{2}}{\mathrm{~d} \dot{y}_{e}}\right)$ and $\left(\frac{\mathrm{d} g_{3}}{\mathrm{~d} \dot{x}_{e}}, \frac{\mathrm{d} g_{3}}{\mathrm{~d} \dot{y}_{e}}\right)$ to be linearly independent. In other words, constraints $g_{2}$ and $g_{3}$ must provide complementary information, that is, must close one DoF each.

In order to determine whether this condition is met or not, more details on $g_{2}$ and $g_{3}$ need to be used. Intuitively, if $g_{2}$ defines the module of the horizontal speed and $g_{3}$ defines its direction, this condition will be met.

\footnotetext{
${ }^{8}$ More information on the APM is provided in part III, and some example values for all constants are provided in table B.1.
} 
Given the descriptions of the generalized constraints that define the horizontal speed in polar coordinates, it can be assumed that

$$
\begin{array}{lll}
\frac{\mathrm{d} g_{2}}{\mathrm{~d} \dot{x}_{e}} \neq 0 & \frac{\mathrm{d} g_{2}}{\mathrm{~d} \dot{y}_{e}}=0 & \text { if }|\cos \chi|>|\sin \chi| \\
\frac{\mathrm{d} g_{2}}{\mathrm{~d} \dot{x}_{e}}=0 & \frac{\mathrm{d} g_{2}}{\mathrm{~d} \dot{y}_{e}} \neq 0 & \text { otherwise }
\end{array}
$$

and also that

$$
\frac{\mathrm{d} g_{3}}{\mathrm{~d} \dot{x}_{e}}=-\sin \chi \quad \frac{\mathrm{d} g_{3}}{\mathrm{~d} \dot{y}_{e}}=\cos \chi
$$

With the assumptions above, it can be stated that (6.34) is non-singular, and therefore (6.31) is also non-singular for any reasonable initial conditions.

In addition to determining whether a DAE can be solved or not, Pryce's structural analysis provides the following information:

- The hidden constraints of the system. Hidden constraints arise when differentiating some algebraic constraints. Pryce's method determines which algebraic constraints must be differentiated in order to obtain all the hidden constraints of the system, and an enlarged system of equations (including differential, algebraic and hidden equations) is built [132]. The enlarged system of equations must be taken into account when defining the consistent initial conditions.

For the set of algebraic constraints analysed in this section, there are three hidden constraints (which are obtained from deriving constraints $g_{1}, g_{2}$, and $\left.g_{3}\right)$ :

$$
\begin{aligned}
\frac{\mathrm{d} g_{1}\left(\dot{z}_{e}\right)}{\mathrm{d} t} & =\frac{\mathrm{d} f_{1}(\mathbf{X}, t)}{\mathrm{d} t} \\
\frac{\mathrm{d} g_{2}\left(\dot{x}_{e}, \dot{y}_{e}\right)}{\mathrm{d} t} & =\frac{\mathrm{d} f_{2}(\mathbf{X}, t)}{\mathrm{d} t} \\
\frac{\mathrm{d} g_{3}\left(\dot{x}_{e}, \dot{y}_{e}, f_{3}(\mathbf{X}, t)\right)}{\mathrm{d} t} & =0
\end{aligned}
$$

- The initial degrees of freedom, D, of the DAE system. During the initialisation of the DAE problem (that is, the process of finding a set of consistent initial conditions), $D$ indicates the number of variables that must be provided by the user, as they are not explicitly or implicitly defined by the constraints.

In the analysis of the set of constraints performed in this section, $D=3$.

- An upper bound to the differential index $\left(\nu_{d}\right)$ of the system. The differential index provides an understanding of the distance between the DAE and its related ODE. It is defined in [143] as:

The minimum number of times that all or part of the DAE system must be differentiated with respect to $t$ in order to determine $\dot{\mathbf{X}}$ as a function of $\mathbf{X}$ and $t$. 
This means that after differentiating all or part of the DAEs at most $\nu_{d}$ times, an ODE system of the form

$$
\dot{\mathbf{X}}=\mathcal{F}^{\prime}(\mathbf{X}, \mathbf{u}, t)
$$

is obtained. Any solution to the DAE also satisfies the ODE. Obviously, not every solution of the ODE is a solution of the DAE. In general, the higher the differential index of the DAE, the more difficult it is to solve it numerically. For that matter, ODEs can be considered as DAEs of differential index 0 .

For the set of algebraic constraint analysed in this section, $\nu_{d} \leq 1$.

\section{Other combinations of constraints}

A thorough analysis would require to repeat Pryce's structural analysis for every possible combination of constraints. As stated before, this is out of the scope of this thesis, and only the combinations considered indispensable have been analysed. The results of these analyses are condensed in table 6.4.

\begin{tabular}{|c|c|c|c|}
\hline$\#$ & $\begin{array}{l}\text { Representative } \\
\text { constraints }\end{array}$ & $\begin{array}{l}\text { Affected } \\
\text { variables }\end{array}$ & Results \\
\hline \multirow{4}{*}{1} & $g_{1}\left(\dot{z}_{e}\right)=0$ & Vertical speed & Solvability: solvable \\
\hline & $g_{2}\left(\dot{x}_{e}, \dot{y}_{e}\right)=0$ & Horizontal speed (module) & Differential index: $\nu_{d} \leq 1$ \\
\hline & $g_{3}\left(\dot{x}_{e}, \dot{y}_{e}\right)=0$ & Horizontal speed (direction) & Number of hidden constraints: 3 \\
\hline & $g_{4}(\psi)=0$ & Yaw angle & Initial DoFs: $D=3$ \\
\hline \multirow{4}{*}{2} & $g_{1}\left(\Omega_{T}\right)=0$ & Thrust & Solvability: solvable \\
\hline & $g_{2}\left(\dot{x}_{e}, \dot{y}_{e}\right)=0$ & Horizontal speed (module) & Differential index: $\nu_{d} \leq 1$ \\
\hline & $g_{3}\left(\dot{x}_{e}, \dot{y}_{e}\right)=0$ & Horizontal speed (direction) & Number of hidden constraints: 2 \\
\hline & $g_{4}(\psi)=0$ & Yaw angle & Initial DoFs: $D=4$ \\
\hline \multirow{4}{*}{3} & $g_{1}\left(z_{e}\right)=0$ & Altitude & Solvability: solvable \\
\hline & $g_{2}\left(\dot{x}_{e}, \dot{y}_{e}\right)=0$ & Horizontal speed (module) & Differential index: $\nu_{d} \leq 2$ \\
\hline & $g_{3}\left(\dot{x}_{e}, \dot{y}_{e}\right)=0$ & Horizontal speed (direction) & Number of hidden constraints: 4 \\
\hline & $g_{4}(\psi)=0$ & Yaw angle & Initial DoFs: $D=2$ \\
\hline \multirow{4}{*}{4} & $g_{1}\left(\dot{z}_{e}\right)=0$ & Vertical speed & Solvability: solvable \\
\hline & $g_{2}(\phi)=0$ & Roll angle & Differential index: $\nu_{d} \leq 1$ \\
\hline & $g_{3}(\theta)=0$ & Pitch angle & Number of hidden constraints: 1 \\
\hline & $g_{4}(\psi)=0$ & Yaw angle & Initial DoFs: $D=5$ \\
\hline \multirow{4}{*}{5} & $g_{1}\left(\Omega_{T}\right)=0$ & Thrust & Solvability: solvable \\
\hline & $g_{2}(\phi)=0$ & Roll angle & Differential index: $\nu_{d} \leq 0$ \\
\hline & $g_{3}(\theta)=0$ & Pitch angle & Number of hidden constraints: 0 \\
\hline & $g_{4}(\psi)=0$ & Yaw angle & Initial DoFs: $D=6$ \\
\hline \multirow{4}{*}{0} & $g_{1}\left(z_{e}\right)=0$ & Altitude & Solvability: solvable \\
\hline & $g_{2}(\phi)=0$ & Roll angle & Differential index: $\nu_{d} \leq 2$ \\
\hline & $g_{3}(\theta)=0$ & Pitch angle & Number of hidden constraints: 2 \\
\hline & $g_{4}(\psi)=0$ & Yaw angle & Initial DoFs: $D=4$ \\
\hline
\end{tabular}

Table 6.4: Results of Pryce's structural analysis for several combinations of constraints. 
All the combinations in table 6.4 are solvable, and most of them are selfexplanatory. A short summary of what each set of constraints does is provided below:

1. A flight defined by the horizontal speed in polar coordinates, vertical speed, and yaw.

2. A flight defined by the horizontal speed in polar coordinates, thrust, and yaw.

3. A flight defined by the horizontal speed in polar coordinates, altitude, and yaw.

4. A flight defined by the vertical speed, roll, pitch and yaw.

5. A flight defined by the thrust, roll, pitch and yaw.

6. A flight defined by the altitude, roll, pitch and yaw.

From these results, some simple rules can be set to the constraints in the QR-AIDL to guarantee that the resulting DAE is solvable. The QR-AIDL will have four threads of execution, of which:

- One thread is dedicated to the yaw angle of the quadrotor.

- Two threads define the horizontal profile of the quadrotor. It can be defined either by constraining the horizontal speed or by constraining the roll and pitch angles.

- One thread defines the altitude of the quadrotor. It can be defined either by explicitly constraining the altitude, the vertical speed, or the thrust.

In the next sections, the QR-AIDL is specified attending to these rules.

\subsection{Language description}

The QR-AIDL derived in this chapter is analogous to the FW-AIDL described in appendix A. Therefore, this section will use the concepts, definitions, and terminology defined there in order to describe the QR-AIDL. The user should familiarise with those before continuing through this chapter.

As any other variant of the AIDL, the QR-AIDL comprises a number of instructions and the rules to combine them in order to form valid descriptions of the flight.

\subsubsection{Instructions}

Instructions are the elements of the AIDL that represent the algebraic constraints that close the mathematical DoFs of the system of equations. These algebraic constraints are denoted the effect of the instruction. In addition, instructions define an execution interval that indicates the interval of time during which the constraint remains active. The execution interval is defined by means 


\begin{tabular}{ll|ll} 
Code & Instruction & Code & Instruction \\
\hline \hline TL & throttle law & PL & pitch law \\
HT & hold throttle & HP & hold pitch \\
OLT & open loop throttle & OLP & open loop pitch \\
RL & roll law & YL & yaw law \\
HR & hold roll & HY & hold yaw \\
OLR & open loop roll & OLY & open loop yaw \\
CL & course law & HSL & horizontal speed law \\
HC & hold course & HHS & hold horizontal speed \\
AL & altitude law & VSL & vertical speed law \\
HA & hold altitude & HVS & hold vertical speed
\end{tabular}

Table 6.5: AIDL instructions for quadrotor aircraft.

of triggers, that is, conditions that indicate a transition from one instruction to another.

The triggers available in the QR-AIDL are the same as in the FW-AIDL. However, since there are no 'set' instructions, default triggers are unnecessary. This leaves with the following types of trigger (more detailed descriptions can be found in in section A.1.1):

1. Fixed triggers are used when the end condition is a particular time.

2. Floating triggers are used when the end of the instruction is a condition on the state and/or control variables.

3. Linked triggers are used when the end condition is determined by another instruction's trigger.

4. Auto triggers are used when the end of the instruction is a condition on the state and/or control variables at a future time.

The QR-AIDL defines the set of effects summarized in table 6.5, which can be classified in the same categories described in section A.1.1. Each of the effects in table 6.5 has an associated algebraic constraint and a series of parameters. These parameters are necessary for completely defining the instruction. In QRAIDL parameters must be of one of the following types:

- Function parameters define the value of a magnitude as a function of one or more state and/or control variables. For example, $f(\mathbf{X}, t) \equiv 2 \cdot \dot{z}_{e}$ is a function parameter equal to twice the vertical speed.

- Magnitude parameters correspond to a particular case of function parameter where the magnitude doesn't depend on any state variable, i.e., it represents a constant value. In practice, most quadrotor operations can be described using magnitude parameters, and function parameters are unnecessary. For example, $f(\mathbf{X}, t) \equiv 5 \mathrm{~m} / \mathrm{s}$ is a magnitude parameter equal to five meters per second.

- Specifier parameters provide additional information to the instruction in the form of a discrete value. In the QR-AIDL only one type of specifier is available, and it is used to indicate the reference frame used in 
the instruction: either an Earth-fixed frame (indicated using the specifier EARTH) or a body-fixed Earth-normal reference frame (indicated using the specifier AIR).

An instruction in QR-AIDL is specified using the corresponding effect code, followed by all the necessary parameters and the end trigger that indicates the end of the instruction. For example:

$\operatorname{VSL}\left(\mathbf{E A R T H}, 2 \mathrm{~m} / \mathrm{s}, z_{e} \geq 100 \mathrm{~m}\right)$

describes a vertical speed law instruction with a specifier parameter determining the reference frame (an Earth-fixed frame, $E$ ), a magnitude parameter governing the vertical speed $(2 \mathrm{~m} / \mathrm{s})$, and a trigger indicating when the instruction finishes. More details on this notation are included in appendix D.

A complete description of each effect follows.

\section{Throttle-related effects}

Some of the effects in the QR-AIDL constrain the rotation speed of the propellers $\Omega_{T}$.

1. The throttle law (TL) effect represents the equation

$$
\Omega_{T}=f(\mathbf{X}, t)
$$

where $f(\mathbf{X}, t)$ is a function or magnitude parameter that must be provided by the user.

2. The hold throttle (HT) effect is a particular type of TL effect with $f(\mathbf{X}, t)=\Omega_{T, 0}$, where $\Omega_{T, 0}$ is the value of $\Omega_{T}$ at the start of the execution interval of the instruction.

3. The open loop throttle (OLT) effect is a particular type of TL effect where $f(\mathbf{X}, t)=f(t), f(t)$ being provided by the user.

\section{Rotation-related effects}

Some effects constrain the rotation of the quadrotor along the $x_{b}, y_{b}$, and $z_{b}$ axes, denoted roll $(\phi)$, pitch $(\theta)$, and yaw $(\psi)$ angles, respectively.

4. The roll law (RL), pitch law (PL), and yaw law (YL) effects represent equations

$$
\begin{aligned}
\phi & =f(\mathbf{X}, t) \\
\theta & =f(\mathbf{X}, t) \\
\psi & =f(\mathbf{X}, t)
\end{aligned}
$$

respectively, where $f(\mathbf{X}, t)$ is a function or magnitude parameter that must be provided by the user. 
5. The hold roll (HR), hold pitch (HP), and hold yaw $(\mathbf{H Y})$ effects are particular types of the corresponding law effects with

$$
\begin{aligned}
& f(\mathbf{X}, t)=\phi_{0} \\
& f(\mathbf{X}, t)=\theta_{0} \\
& f(\mathbf{X}, t)=\psi_{0}
\end{aligned}
$$

where $\phi_{0}, \theta_{0}$, and $\psi_{0}$ are the values of $\phi, \theta$, and $\psi$, respectively, at the start of the execution interval of the instruction.

6. The open loop roll (OLR), open loop pitch ( OLP), and open loop yaw (OLY) instructions are particular types of the corresponding law effects where $f(\mathbf{X}, t)=f(t), f(t)$ being provided by the user.

\section{Altitude-related effects}

There are two effects related to the altitude ${ }^{9}\left(z_{e}\right)$ of the quadrotor:

7. The altitude law (AL) effect represents the equation

$$
z_{e}=f(\mathbf{X}, t)
$$

where $f(\mathbf{X}, t)$ is a function or magnitude parameter that must be provided by the user.

8. The hold altitude (HA) effect is a particular type of $\mathbf{A L}$ effect with $f(\mathbf{X}, t)=z_{e, 0}$, where $z_{e, 0}$ is the value of $z_{e}$ at the start of the execution interval of the instruction.

\section{Vertical speed-related effects}

Instructions related to the vertical speed of the quadrotor require a parameter specifying the reference frame of the speed. There are two options allowed:

- Vertical speed with respect to an Earth-fixed frame $E$ (EARTH). In this case, the left hand side of the equations is:

$$
g\left(\dot{z}_{e}\right)=\dot{z}_{e}
$$

- Vertical speed with respect to a body-fixed Earth-normal reference frame $A$ (AIR). This reference frame has its origin fixed to the quadrotor's center of mass, but has the same orientation of $E$. In this case, the left hand side of the equations is:

$$
g\left(\dot{z}_{e}\right)=\dot{z}_{a}=\dot{z}_{e}-w_{z}
$$

where $\dot{w}_{z}$ is the local wind speed along $\dot{z}_{a}$ axis (or equivalently $\dot{z}_{e}$ axis).

${ }^{9}$ All the altitudes considered in the QR-AIDL are geometric altitudes. Barometric altitudes should be converted to geometric altitudes before use, and may not be sufficiently precise for some applications of the QR-AIDL. 
There are two effects related to the vertical speed of the quadrotor:

9. The vertical speed law (VSL) effect represents the equation

$$
g\left(\dot{z}_{e}\right)=f(\mathbf{X}, t)
$$

where $f(\mathbf{X}, t)$ is a function or magnitude parameter that must be provided by the user.

10. The hold vertical speed (HVS) effect is a particular type of VSL effect with $f(\mathbf{X}, t)=g\left(\dot{z}_{e, 0}\right)$, where $\dot{z}_{e, 0}$ is the value of $\dot{z}_{e}$ at the start of the execution interval of the instruction.

\section{Path angle-related effects}

The effects defining the path angle of the quadrotor are a particular type of vertical speed-related effects. There are two effects related to the path angle:

11. The path angle law (PAL) effect represents the equation

$$
\dot{z}_{e}=v_{e} \sin f(\mathbf{X}, t)
$$

if the Earth-fixed reference frame $E$ (EARTH) is chosen, and

$$
\dot{z}_{a}=v_{a} \sin f(\mathbf{X}, t)
$$

if the body-fixed Earth-normal reference frame $A$ (AIR) is chosen. The function $f(\mathbf{X}, t)$ governs either the geometric path angle $\gamma_{e}$ (in the first case) or the aerodynamic path angle $\gamma_{a}$ (in the second case); and it is a function or magnitude parameter that must be provided by the user. The geometric speed $v_{e}$ and aerodynamic speed $v_{a}$ can be computed as

$$
\begin{aligned}
v_{e} & =\sqrt{\dot{x}_{e}^{2}+\dot{y}_{e}^{2}+\dot{z}_{e}^{2}} \\
v_{a} & =\sqrt{\dot{x}_{a}^{2}+\dot{y}_{a}^{2}+\dot{z}_{a}^{2}} \\
& =\sqrt{\left(\dot{x}_{e}-w_{x}\right)^{2}+\left(\dot{y}_{e}-w_{y}\right)^{2}+\left(\dot{z}_{e}-w_{z}\right)^{2}}
\end{aligned}
$$

12. The hold path angle (HPA) effect is a particular type of PAL effect with $f(\mathbf{X}, t)=\gamma_{e, 0}$ or $f(\mathbf{X}, t)=\gamma_{a, 0}$ (depending on the reference frame used), where $\gamma_{e, 0}$ and $\gamma_{a, 0}$ are the values of $\gamma_{e}$ and $\gamma_{a}$ at the start of the execution interval of the instruction.

\section{Course-related effects}

Effects related to the course of the quadrotor also require the reference frame to be specified.

- If the Earth-fixed frame $E$ (EARTH) is chosen, then the constraint's equation is:

$$
\dot{y}_{e} \cos f(\mathbf{X}, t)-\dot{x}_{e} \sin f(\mathbf{X}, t)=0
$$

where $f(\mathbf{X}, t)$ describes the geometric course $\chi_{e}$ of the aircraft.

$$
\chi_{e}=\operatorname{atan} 2\left(\dot{y}_{e}, \dot{x}_{e}\right)
$$


- If the body-fixed Earth-normal reference frame $A$ (AIR) is chosen, then the equation is:

$$
\dot{y}_{a} \cos f(\mathbf{X}, t)-\dot{x}_{a} \sin f(\mathbf{X}, t)=0
$$

or equivalently,

$$
\left(\dot{y}_{e}-w_{y}\right) \cos f(\mathbf{X}, t)-\left(\dot{x}_{e}-w_{x}\right) \sin f(\mathbf{X}, t)=0
$$

where $w_{x}$ and $w_{y}$ are the local wind speeds along the $x_{e}$ and $y_{e}$ axes, respectively; and $f(\mathbf{X}, t)$ describes the aerodynamic course $\chi_{a}$ of the aircraft.

$$
\chi_{a}=\operatorname{atan} 2\left(\dot{y}_{a}, \dot{x}_{a}\right)=\operatorname{atan} 2\left(\dot{y}_{e}-w_{y}, \dot{x}_{e}-w_{x}\right)
$$

There are two effects related to the course:

13. The course law $(\mathbf{C L})$ effect represents the corresponding equation of the ones described above, where $f(\mathbf{X}, t)$ is a function or magnitude parameter that must be provided by the user.

14. The hold course (HC) effect is a particular type of $\mathbf{C L}$ effect. If the EARTH specifier is used, then $f(\mathbf{X}, t)=\chi_{e, 0}$, whereas if the AIR specifier is used, then $f(\mathbf{X}, t)=\chi_{a, 0}$, where $\chi_{e, 0}$ and $\chi_{a, 0}$ are the values of $\chi_{e}$ and $\chi_{a}$, respectively, at the start of the execution interval (assuming the initial horizontal speed is non-zero).

\section{Horizontal speed-related effects}

As in other effect groups described above, the reference frame is relevant in effects related to the horizontal speed.

- If the Earth-fixed frame $E$ (EARTH) is chosen, then the constraint's equation is:

$$
g\left(\dot{x}_{e}, \dot{y}_{e}\right) \equiv \begin{cases}\dot{x}_{e}=f(\mathbf{X}, t) \cos \chi_{e} & \text { if }\left|\cos \chi_{e}\right|>\left|\sin \chi_{e}\right| \\ \dot{y}_{e}=f(\mathbf{X}, t) \sin \chi_{e} & \text { otherwise }\end{cases}
$$

where $f(\mathbf{X}, t)$ describes the horizontal speed of the aircraft with respect to $E, v_{g}$ :

$$
v_{g}=\sqrt{\dot{x}_{e}^{2}+\dot{y}_{e}^{2}}
$$

- If the body-fixed Earth-normal reference frame $A$ (AIR) is chosen, then the constraint's equation is:

$$
g\left(\dot{x}_{e}, \dot{y}_{e}\right) \equiv \begin{cases}\dot{x}_{a} \equiv \dot{x}_{e}-w_{x}=f(\mathbf{X}, t) \cos \chi_{a} & \text { if }\left|\cos \chi_{a}\right|>\left|\sin \chi_{a}\right| \\ \dot{y}_{a} \equiv \dot{y}_{e}-w_{y}=f(\mathbf{X}, t) \sin \chi_{a} & \text { otherwise }\end{cases}
$$

where $f(\mathbf{X}, t)$ describes the horizontal speed of the aircraft with respect to $A, v_{h}$ :

$$
v_{h}=\sqrt{\dot{x}_{a}^{2}+\dot{y}_{a}^{2}}=\sqrt{\left(\dot{x}_{e}-w_{x}\right)^{2}+\left(\dot{y}_{e}-w_{y}\right)^{2}}
$$




\begin{tabular}{l|l|l} 
Profile & Instructions & Thread \\
\hline \hline Vertical & AL, HA, VSL, HVS, PAL, HPA & \multirow{2}{*}{ Longitudinal } \\
\hline Propulsive & TL, HT, OLT & \multirow{2}{*}{ Lateral (1st or 2nd) } \\
\hline Lateral & HSL, HHS, CL, HC & Orientation
\end{tabular}

Table 6.6: Instruction profiles and related threads.

There are two effects related to the horizontal speed:

15. The horizontal speed law (HSL) effect represents the corresponding equation of the ones described above, where $f(\mathbf{X}, t)$ is a function or magnitude parameter that must be provided by the user.

16. The hold horizontal (HHS) effect is a particular type of HSL effect. If the EARTH specifier is used, then $f(\mathbf{X}, t)=v_{g, 0}$, whereas if the AIR specifier is used, then $f(\mathbf{X}, t)=v_{h, 0}$, where $v_{g, 0}$ and $v_{h, 0}$ are the values of $v_{g}$ and $v_{h}$, respectively, at the start of the execution interval.

\subsubsection{Intent}

In addition to the collection of instructions, the QR-AIDL includes a number of rules designed to ensure that a flight described in this language represents a guidance strategy that closes the four mathematical DoFs of the system and provides an unambiguous description of the flight. This section describes the rules required to form a valid QR-AIDL intent, which are analogous to those of the FW-AIDL (see section A.1.2).

In order to close the four mathematical DoFs, the same number of instructions must be active at any time. In the QR-AIDL, this is achieved by requiring four parallel execution threads. Each execution thread contains a sequence of one or more instructions. These instructions are executed sequentially, so that the end of the execution interval of one instruction in a thread coincides with the start of the execution interval of the next instruction in the same thread. The execution interval of the first instruction in each thread starts at the initial conditions of the flight. In order for the intent to be valid, all the execution threads must start and end simultaneously. Since all the threads start at the initial conditions, they all start simultaneously. To ensure that they all end simultaneously, there must be a single trigger, known as the master trigger, defining the end of the intent: one of the threads must end with the master trigger, while the others must have linked triggers pointing to it.

There are also some restrictions regarding what kind of instructions can be allocated to each thread, which are summarized in table 6.6. The four threads can be categorized as:

- One longitudinal thread, which may contain instructions related to the altitude, vertical speed, path angle and thrust of the quadrotor.

- Two lateral threads, which may contain a combination of instructions related to the roll and pitch, or a combination of instructions related to the 
course and horizontal speed. One intent can use both types of combinations to define its lateral thread, but instructions of different combinations cannot be active simultaneously. For example, an intent with a CL and a OLR instruction simultaneously active isn't a valid QR-AIDL word.

- One orientation thread, which contains yaw-related instructions.

Using the rules above, it is possible to combine QR-AIDL instructions to form valid intent.

\subsection{Graphical representation}

A graphical notation for representing FW-AIDL intent was introduced in [16]. An analogous notation is presented here that enables representing QRAIDL intent graphically. Any QR-AIDL intent represented using this graphical notation must comply with all the rules described in the previous section.

Instructions are represented as rectangles containing a description of its effect and parameters, using the notation in appendix D. These rectangles may have a background colour, which depends on the effect of the instruction being represented. Each effect is assigned a particular colour depending on the variables it affects, as shown in table 6.7. The end trigger of the instruction is represented using the same notation as depicted in figure A.1.

\begin{tabular}{l|l} 
Group & Effects \\
\hline \hline Altitude & AL, HA \\
Vertical speed & VSL, HVS \\
Path angle & PAL, HPA \\
Throttle & TL, HT, OLT \\
Horizontal speed & HSL, HHS \\
Course & CL, HC \\
Roll & RL, HR, OLR \\
Pitch & PL, HP, OLP \\
Yaw & YL, HY, OLY
\end{tabular}

Table 6.7: Effect groups and assigned colours.

For example, a hold course instruction using an Earth-fixed reference frame with a floating trigger is shown in figure 6.2. Multiple instructions in the same thread are represented by concatenating the rectangles corresponding to each instruction, from left to right. An intent is represented as four parallel rows of instructions. Several examples of complete QR-AIDL intent are provided in the next section.

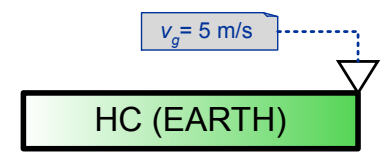

Figure 6.2: QR-AIDL instruction in graphical notation. 


\subsection{Examples}

A simple QR-AIDL intent is shown in figure 6.3. In this intent, only one instruction is included per thread. The end of the intent is the fixed trigger (with a condition $t=60 \mathrm{~s}$ ), which acts as the master trigger, with all other instructions having linked triggers pointing (directly or indirectly) to it. Also, in this first example, no instruction parameters are necessary, as the effects used do not require any. This intent will keep the four control variables $\left(\Omega_{T}, \phi, \theta\right.$, and $\psi$ ) constant. The initial values of these variables are obtained from the initial conditions.

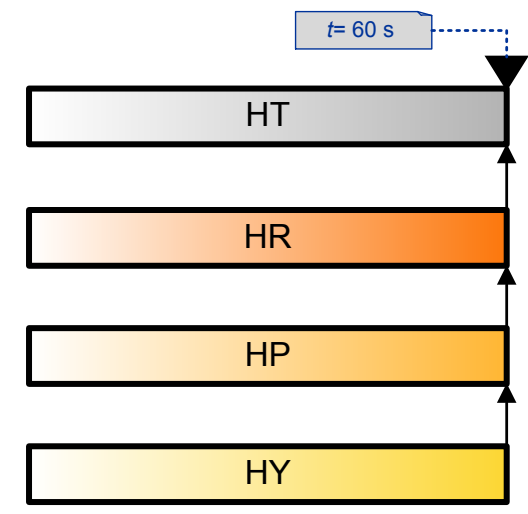

Figure 6.3: Simple QR-AIDL intent.

Of course, the QR-AIDL allows the definition of more complex flights. Another example is provided in figure 6.4. In this case, several types of instructions are used. The quadrotor flies with a constant horizontal speed of $4 \mathrm{~m} / \mathrm{s}$ and a path angle of 12 degrees towards a certain point (which is reached by moving with a fixed geometric course of 22.5 degrees). Once the point is reached, the quadrotor will slowly descend to 50 meters while staying in the same horizontal position and keeping the yaw angle constant as well. Finally, the last operation of this intent sets the attitude of the quadrotor (i.e., the roll and pitch angles) while the altitude is kept constant, which will produce a horizontal acceleration. The intent finishes when the condition in the master trigger is met, that is, the ground speed reaches $4 \mathrm{~m} / \mathrm{s}$.

\subsection{Final words}

This chapter has explored the feasibility of designing a variant of the AIDL that can be used to unambiguously describe quadrotor flights. The steps followed by BR\&TE $[16,144]$ have been reproduced in order to derive the language from the AMM. However, in the case of quadrotors, additional problems had to be dealt with.

First, the choice of an AMM was not straightforward. Modelling the motion of a quadrotor has traditionally been targeted at developing better control systems for these vehicles. Therefore, most of the literature uses a 6-DoFs (class 


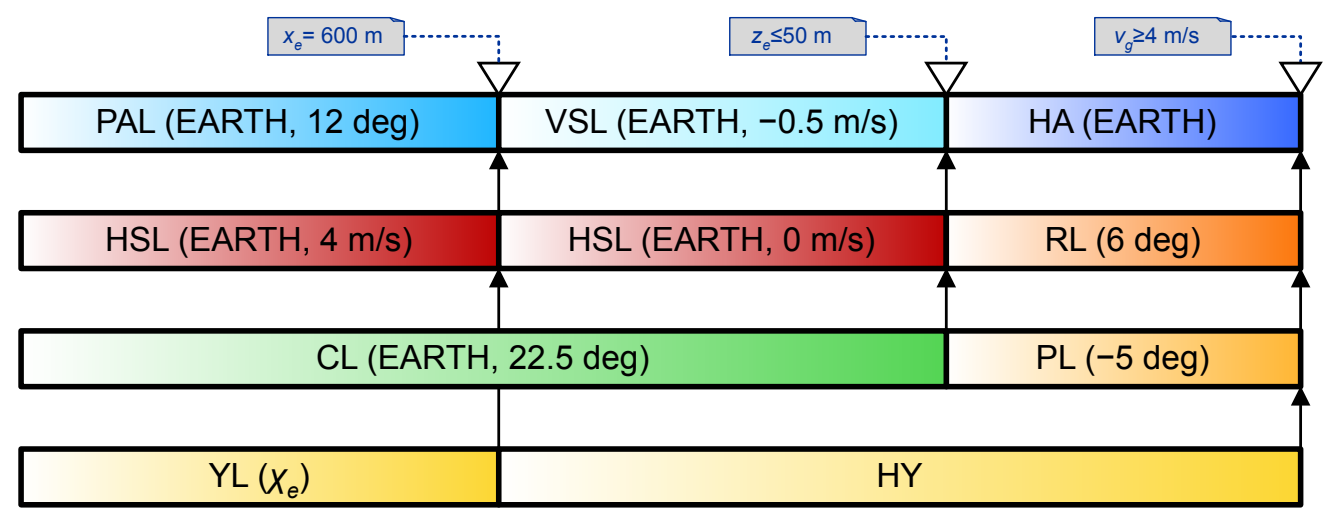

Figure 6.4: QR-AIDL intent.

A) model. These highly detailed models were not adequate for the development of the QR-AIDL, so a simpler 3-DoFs (class B) model was developed from the 6-DoFs model.

There are also assumptions that are reasonable for a fixed-wing commercial aircraft, but they can't be assumed when describing guidance modes for a quadrotor. For example, in the derivation of the FW-AIDL, airspeeds were assumed to always be above some threshold. This assumption is not valid in the case of a quadrotor, as it can hover without changing its relative position with respect to the air mass, and also climb with a path angle of 90 degrees. This led to some problems that were overcome in most cases by carefully choosing the equations that defined every guidance mode. Other guidance modes have not been included in the QR-AIDL, as no solution was found to the corner case where $v_{g}=0$. The exploration of alternative equations for defining these guidance modes is left as future work.

A complete solvability analysis, which would have required analysing all the possible combinations of generic constraints, has not been performed. This is also related with the problem above: the solvability of some combinations of generic constraints can't be adequately assessed until the corresponding guidance modes are further studied.

The problem of finding some consistent initial conditions has also been overlooked in this chapter. A DAE system requires that the initial conditions are consistent with all the constraints in the system (including hidden constraints). This problem is addressed in [16] for the FW-AIDL, and the approach to find a solution for the QR-AIDL would be essentially the same.

It is not in the scope of this thesis to analyse and develop the QR-AIDL to the same extent as the FW-AIDL was. The purpose of this chapter was to demonstrate the feasibility of the QR-AIDL. This feasibility has been proven by developing a language that is sufficient to describe any quadrotor trajectory of interest to this thesis. New instructions could be added to the QR-AIDL if further developed. 


\section{Chapter 7}

\section{Intent composite description language}

The QR-AIDL described in the previous chapter and the FW-AIDL described in appendix A were designed to completely describe aircraft trajectories, closing all DoFs. While this is one of the most important features of those languages, it may also make those languages not suitable for other uses, where it is desirable to leave some DoFs open. This chapter introduces a new language, developed as part of this thesis in collaboration with BR\&TE, that enables less detailed descriptions of aircraft trajectories, among other new features.

The intent composite description language (ICDL) $[128,145]$ is a formal language based on the AIDL (any of its variants, including the FW-AIDL and the QR-AIDL). This language provides the means to arrange AIDL instructions in composites, which provide an additional layer of abstraction. The advantages of working with composites are manifold:

- The ICDL is more flexible than the AIDL in that it doesn't require the intent to be completely and unambiguously defined. Thus, a composite may only define some DoFs, and some of the AIDL instruction parameters may not be completely defined.

- The syntax of the ICDL provides several operations to allow generating long and complex flights from simple composites. These operations make easier for human operators to understand the resulting intent.

- Some of the ICDL operations provide additional semantics. In particular, they allow defining conditions, which are evaluated in execution time (i.e., when the actual trajectory is flown, either in a real or simulated environment), with different aircraft behaviours depending on how these conditions evaluate.

- There are several combinations of AIDL instructions that appear recurrently in flights. For example, the AIDL instructions that describe a level flight will appear in virtually every flight, but perhaps with different instruction parameters. The ICDL provides templating mechanisms that enable reuse of recurrent combinations of AIDL instructions. 
These features of the ICDL make it a language that is more powerful and easier to understand than the AIDL is. These improvements, however, have a cost: while any intent expressed in the AIDL describes an aircraft trajectory unambiguously, intent expressed in ICDL - in general - don't. As a consequence, intent can't be univocally translated from ICDL into AIDL.

\subsection{Language description}

All valid sentences in the ICDL are denoted composites. Composites can be seen as a way of expressing intent. Unlike in AIDL, intent expressed using ICDL can provide an ambiguous description of an aircraft's trajectory. That is, there are potentially many different trajectories matching the description provided by an ICDL intent.

The simplest composite that can be built in ICDL is an elementary composite. Elementary composites can be built as a combination of AIDL instructions that closes zero, one, or more DoF of the aircraft motion. In practice, however, elementary composites are most often built from a template. A template represents a common combination of AIDL instructions, with perhaps some undefined instruction parameters and triggers, that is given a meaningful name. An elementary composite can then be built from a template by providing the undefined parameters and triggers.

Using a series of operations on composites, more complex composites can be built. The longitudinal and transversal composition are operations expressing the sequential and simultaneous execution of several composites, respectively. The conditional branching and the conditional loop allow non-linear execution of composites, i.e., allow composites to be executed sequentially in a variable order (which is decided at execution time, depending on some conditions).

All composites comprise an effect and an execution interval. The effect describes - completely or partially - the behaviour of the aircraft while the composite is active. This behaviour is defined by the AIDL instructions that compose the composite. The execution interval defines the time interval where the composite is active. This interval is the mathematical union of the execution intervals of the AIDL instructions that form the composite.

The remainder of this section focuses on describing in depth some of the concepts outlined above. Note that a composite may be used to represent a flight of a fixed-wing aircraft or a quadrotor aircraft. In the former case, the ICDL will use the instructions, state variables, and specifiers from the FWAIDL. In the latter case, the ICDL will use those of the QR-AIDL. Most of the examples provided in this section refer to fixed-wing aircraft, but section 7.3 provides other examples related to quadrotor aircraft.

\subsubsection{Elementary composites}

Elementary composites are built from AIDL instructions, combined using the rules of $\mathrm{AIDL}^{1}$, but allowing some threads to be empty, and replacing some

\footnotetext{
${ }^{1}$ Rules for the FW-AIDL are provided in [16], whereas the rules of the QR-AIDL were described in section 6.2.2.
} 
AIDL instruction parameters with ICDL parameters. There are two types of ICDL parameters: intervals and sets. Interval parameters replace AIDL magnitudes, and set parameters replace AIDL specifiers. While AIDL instruction parameters and ICDL parameters are very similar semantically, the former need to provide one specific value, whereas the latter allow a more ambiguous definition.

Interval parameters represent dynamic magnitudes (state or control variables) that can be set to any value between a maximum and a minimum. Intervals have a minimum value $m$, a maximum value $M$, and a default value $v$ such that $m \leq v \leq M$. The three values in a particular interval refer to the same magnitude type, which may be any of the magnitude types defined in the AIDL. Intervals can be seen as an extension of AIDL magnitudes where, in addition to its value, a continuous range of possible values is provided. Whenever $v=m=M$, the interval is closed and therefore it is equivalent to an AIDL magnitude.

Using the notation of appendix D, an interval parameter can be expressed as $v: m . . M$. The interval examples below use this notation:

- $240 \mathrm{kt}: 220 . .260$ : a speed between 220 and 260 knots, with a default value of 240 knots.

- 1.45 rad: an angle of 1.45 radians. When only one value is provided, it is the minimum, maximum and default value.

Set parameters represent a collection of AIDL specifiers. These sets must contain at least one element, and all elements must be of the same AIDL specifier type. The advantage of replacing AIDL specifiers with set parameters is that several possible AIDL specifiers can be included, instead of just one.

In the notation of appendix D, sets are expressed as an enumeration of all its elements, separated by commas and within square brackets. Some examples of valid parameter sets are:

- [GEO, PRE]: contains geometric altitude (GEO) and geopotential pressure altitude (PRE).

- $[\mathrm{MCMB}]$ : contains only one element, maximum climb regime (MCMB), a named thrust operational mode.

The idea behind interval and set parameters is to leave some details of the intent open for later refinement or adaptation to specific situations. In a first step, the composite may be specified using intervals (or sets) of valid values and then, in a later step, the optimum values - according to certain criterionwithin the intervals (or sets) may be chosen.

Apart from the obligation to have instructions in all AIDL threads, all the other rules in AIDL apply when combining AIDL instructions to form elementary composites. In particular, all non-empty execution threads need to start and end simultaneously. Therefore, all non-empty threads in a composite always share the end trigger (the master trigger, as explained in chapter 6), which also becomes the end trigger of the composite. Figure 7.1 shows a combination of 


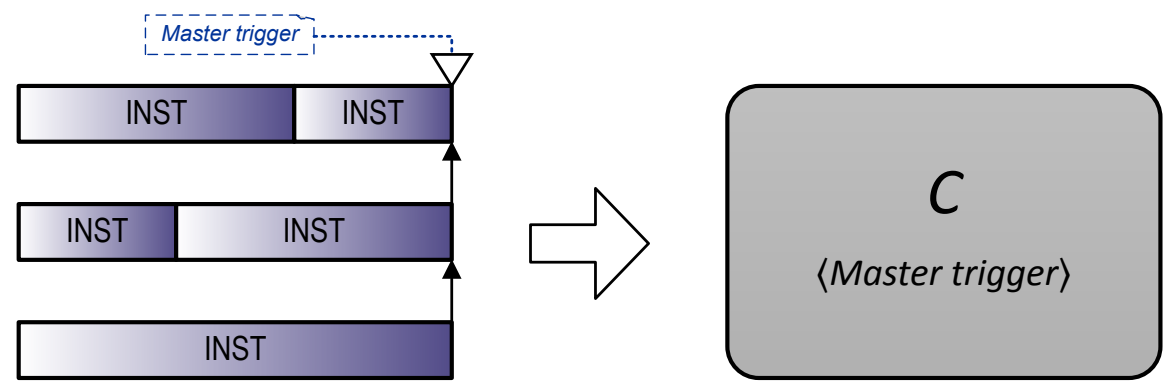

Figure 7.1: Elementary composite built from the combination of AIDL instructions.

AIDL instructions with a master trigger, and how this combination can be interpreted as an elementary composite $C$ (represented as a gray box). The types of trigger that can be used as the end trigger of a composite are detailed next.

\subsubsection{Triggers}

All composites have one end trigger describing a condition that must be met the instant its execution interval ends. In elementary composites, it has been shown that the end trigger of the composites is the master trigger of the combined AIDL instructions. In composites resulting from operations on other composites, the resulting end trigger depends on the particular operation. Composite operations, including details on the resulting end triggers, are addressed in section 7.1.4.

In ICDL, all the trigger types in $\mathrm{AIDL}^{2}$ are available, with the additional flexibility of interval and set parameters. Two additional types of trigger are available in ICDL:

- Waypoint triggers contain a waypoint and a tolerance. The waypoint is a $2 \mathrm{D}$ point of interest (a point of the planned route). It can be defined by the waypoint's name - according to a predefined shared waypoint database, such as the operational context- , or as a pair of $2 \mathrm{D}$ coordinates -latitude and longitude, which can be defined as interval parameters. The tolerance is the maximum allowed divergence of the predicted trajectory from the waypoint position. That is, when the execution interval of a composite with a waypoint trigger ends, the aircraft must be inside a circumference defined by the waypoint (as its center) and the tolerance (as its radius). If no tolerance is explicitly indicated, a pre-established default tolerance is assumed.

Some examples of waypoint triggers, using the notation of appendix D, are:

- CANIS: a waypoint referred to by its name - from the AIP Spain [146] — with the default tolerance.

${ }^{2}$ Including fixed, floating, linked, and auto triggers, described in sections 6.3.1 and A.1.1. 


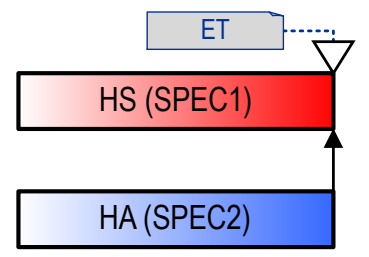

Figure 7.2: 'Level flight' template.

- CANIS[2NM]: the same waypoint with a tolerance of two nautical miles.

- $[27.999722 \mathrm{deg},-14.648056 \mathrm{deg}, 1 \mathrm{NM}]$ : the same waypoint defined through latitude, longitude, and tolerance.

- List triggers contain two or more fixed, floating of waypoint triggers. These triggers cannot be used as the end trigger of an elementary composite; they are implicitly defined by some of the conditional composition operations to be described in section 7.1.4.

\subsubsection{Templates}

As stated in chapter 5, elementary composites are often built so that they represent an understandable behaviour, such as 'maintain a level flight', 'keep clean configuration' or 'follow a lateral path'. The same elementary composites can be used repeatedly, with only small changes in their end triggers or their parameters. Therefore, tools that enable reuse of these recurrent combinations of AIDL instructions become a very useful asset. These tools are the ICDL templates.

\section{Simple templates}

In its simplest form, an ICDL template is an elementary composite where some parameters are left blank. In particular, the end trigger of the composite is usually one of these blank (or undefined) parameters. Templates are also given a meaningful name that describes its behaviour. As an example, a 'level flight' template is described next. This template contains the AIDL instructions describing a constant altitude, constant speed flight by a fixed-wing aircraft, as shown in figure 7.2. The hold speed instruction (which commands the aircraft to maintain its speed constant) requires an additional set parameter indicating the possible types of speed (CAS, TAS, IAS, Mach, etc.). This parameter (SPEC1) has been left blank in the template. The hold altitude instruction (which commands the aircraft to maintain a constant altitude) also needs a set parameter indicating the possible types of altitude (geopotential pressure altitude, geometric altitude, etc.). This parameter is also left blank in the template. Finally, the third undefined parameter is the end trigger of the composite (ET). More examples of ICDL templates are provided in appendix C.

To create an elementary composite from a template, it suffices to specify the name of the template and the values for its blank parameters. In the notation of appendix D, the parameters are listed between angle brackets. For example, an 


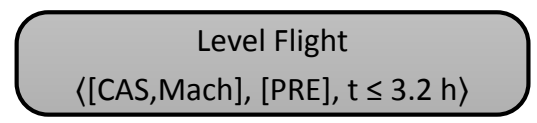

Figure 7.3: Elementary composite created from the 'level flight' template.

elementary composite created from the 'level flight' template would be: 'Level Flight $\langle[\mathrm{CAS}, \mathrm{Mach}],[\mathrm{PRE}], t \leq 3.2 \mathrm{~h}\rangle$ '. The first set parameter replaces SPEC1, and indicates the speed to be held may be either CAS or Mach. The second set parameter replaces SPEC2, and indicates the type of altitude to be held is geopotential pressure altitude (PRE). The last parameter replaces ET, and is a fixed trigger causing the level flight to end after 3.2 hours.

A graphical notation has also been designed, which is discussed in section 7.2. As an example, the 'level flight' composite described above is shown in figure 7.3.

It is also possible to generate an elementary composite with an empty effect to characterize the portions of flight where the aircraft behaviour is totally unspecified. These elementary composites are created from the 'null' template. This template has no AIDL instructions, and its only parameter is the trigger indicating the end of the composite. In the notation used here, the 'null' template name can be omitted, indicating just the trigger parameter in angle brackets (e.g., $\langle$ ENETA $\rangle$ denotes a null composite with a waypoint end trigger).

\section{Algorithmic templates}

Simple templates have a fixed number of AIDL instructions and the undefined parameters must be explicitly provided. However, in some circumstances it is necessary (or simply more convenient) to have more flexibility.

Algorithmic templates can have a variable number of AIDL instructions, and allow undefined parameters of AIDL instructions to be implicitly provided as the result of some calculations. In order to accommodate algorithmic templates, a more general definition of templates must be provided. From now on, a template is defined as a deterministic process with some input and output, where the input is a sequence of parameters (fixed in number and types), and the output is an elementary composite. In simple templates, this process consists simply in assigning each input parameter to the corresponding undefined parameter.

In addition to the types of parameter already described, algorithmic templates can take a list as an input parameter. Lists contain a variable number of elements (waypoints, speeds, specifiers, etc.) of the same type. A parameter list is denoted as $\left\{P_{1}, P_{2}, \ldots, P_{n}\right\}$ where $P_{i}$ is an interval or set parameter. The size of these parameter lists usually determine the number of AIDL instructions of the output composite. For example, the 'geometric path waypoint' template is an algorithmic template that defines the horizontal path as a sequence of waypoints (see section C.1.3). This template takes a list of $n$ waypoints and an end trigger, and provides an elementary composite with $2 n+1$ instructions. These instructions require parameters (AIDL object parameters, such as circular arcs and great circles [16]) that may be computed from the waypoints and their tolerances. 
In the remainder of this thesis, it will be assumed that all elementary composites are created from a template. In particular, the templates in appendix $\mathrm{C}$ will be used.

\subsubsection{Composite operations}

The ICDL defines four operations that allow building more complex composites from simpler ones. These operations are governed by several rules that specify how the effects and end triggers are combined when a specific operation is applied to the operands to generate a new composite. Many of these rules are derived from the underlying rules of the AIDL. The meaning, properties and requirements for those operations are described next. The notation used in this section is detailed in appendix D.

1. The longitudinal composition of two composites $C_{1}$ and $C_{2}$ (noted $C_{1} \oplus C_{2}$ ) implies that the two composites are executed sequentially. That is, $C_{1}$ is executed first and, when finished, $C_{2}$ is executed. Therefore, the execution interval of $C_{1} \oplus C_{2}$ is the concatenation of the execution intervals of $C_{1}$ and $C_{2}$, and the end trigger of $C_{1} \oplus C_{2}$ is the end trigger of $C_{2}$. Longitudinal composition is associative, but not commutative. This operation requires both operands to cover the same AIDL threads.

2. The transversal composition of two composites $C_{1}$ and $C_{2}$ (noted $C_{1} \otimes$ $C_{2}$ ) implies that the composites are executed simultaneously. That is, the execution interval of $C_{1}$ and $C_{2}$ match. To ensure both composites start and end simultaneously, it is necessary that one of the operands' end trigger is a linked trigger to the other operand's end trigger. Therefore, $C_{1}, C_{2}$, and $C_{1} \otimes C_{2}$ have the same execution interval. The end trigger of $C_{1} \otimes C_{2}$ is one of the end triggers of $C_{1}$ or $C_{2}$ : the one that is not a linked trigger. Transversal composition is associative and commutative. This operation requires both operands to cover disjoint sets of AIDL threads.

3. The conditional branching of two composites $C_{1}$ and $C_{2}$, with a boolean condition $\chi$, is noted $\left(C_{1} \mid C_{2}\right)[\chi]$. This operation implies that only one of the operators is executed. That is, if at the start of the execution interval $\chi$ is true, then $C_{1}$ is executed; otherwise $C_{2}$ is executed. The execution interval of $\left(C_{1} \mid C_{2}\right)[\chi]$ is that of the executed operand, and its end trigger is a list trigger containing the end triggers of both $C_{1}$ and $C_{2}$. This operation requires the end triggers of $C_{1}$ and $C_{2}$ to be either fixed, floating, or waypoint triggers. As in the longitudinal composition, operands must cover the same AIDL threads.

4. The conditional loop of a composite $C_{1}$, with a boolean condition $\chi$, and two integers $m$ and $M$ (with $0 \leq m \leq M$ ), is noted $\left(C_{1}\right)[\chi: m: M]$. This operation implies that $C_{1}$ is executed repeatedly a number of times. The condition $\chi$ is evaluated before each execution of $C_{1}$, a repeating process that continues as long as $\chi$ is true, a minimum of $m$ times and a maximum of $M$ times. That is, the numbers $m$ and $M$ force $C_{1}$ to be executed at least $m$ times and at most $M$ times, independently of the evaluation of $\chi$. The end trigger of $\left(C_{1}\right)[\chi: m: M]$ is that of $C_{1}$, and its execution 


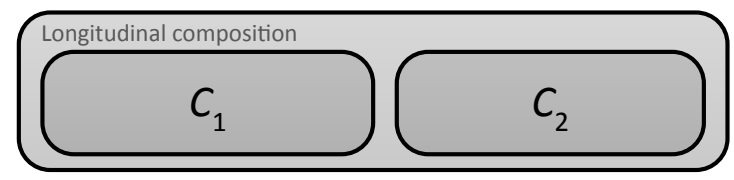

(a) Longitudinal composition: $C_{1} \oplus C_{2}$

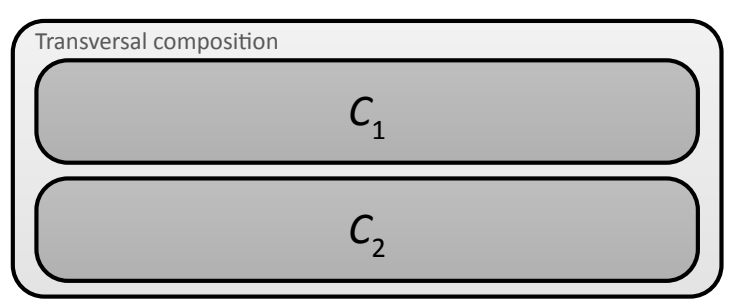

(b) Transversal composition: $C_{1} \otimes C_{2}$

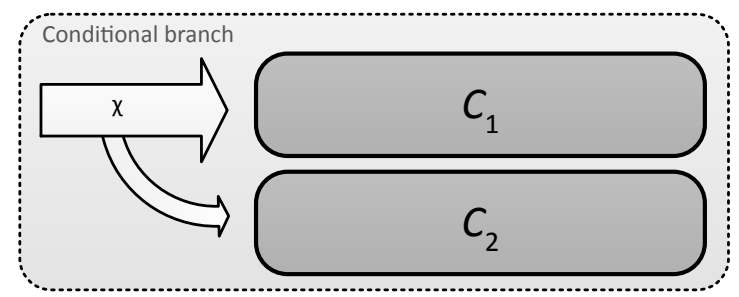

(c) Conditional branch: $\left(C_{1} \mid C_{2}\right)[\chi]$

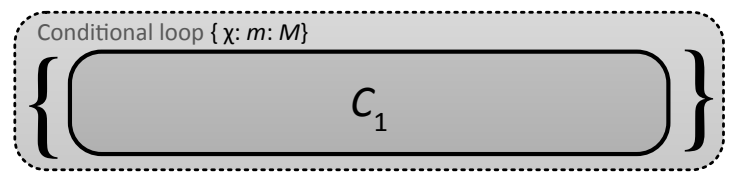

(d) Conditional loop: $\left(C_{1}\right)[\chi: m: M]$

Figure 7.4: Operations on composites.

interval is the concatenation of the execution intervals of every execution of $C_{1}$. This operation requires the end trigger of $C_{1}$ to be either a floating, waypoint or list trigger. Fixed triggers do not make sense in a conditional loop, as they represent a fixed instant of time that cannot be repeated. The integers $m$ and $M$ can be omitted, so the conditional loop can also be noted $\left(C_{1}\right)[\chi]$. In this case, it is assumed that $m=0$ and $M=\infty$.

A graphical notation has also been designed to represent composite operations, shown in figure 7.4. More details on this graphical notation are given in section 7.2.

\section{Conditions}

The conditions that appear in conditional branches and conditional loops are mathematical expressions of the form

$$
f(\mathbf{X}, \mathbf{u}, \boldsymbol{\Delta}, \mathbf{E}, t) \leq 0
$$


where $f$ is a function of the aircraft state vector $\mathbf{X}$, the control vector $\mathbf{u}$, the configuration vector ${ }^{3} \boldsymbol{\Delta}$, the environmental model vector ${ }^{4} \mathbf{E}$, and the time $t$. Conditions are true if the inequation is true at the time when it is evaluated.

For convenience, the notation in (7.1) can be relaxed to allow more readable expressions. Some simple examples of conditions are:

- $d \leq 18 \mathrm{NM}$ : total traversed distance not greater than 18 nautical miles.

- $t \leq 5 \mathrm{~h}$ : total elapsed time not greater than 5 hours.

- $m_{F} \geq 900 \mathrm{~kg}:$ mass of fuel not lower than 900 kilograms.

Note that the state, control and configuration vectors depend on the type of aircraft under consideration, and some of the variables in these examples may not always be available. For example, electrical vehicles don't have a mass of fuel $\left(m_{F}\right)$.

\section{Additional rules}

In addition to the rules described earlier in this section, some rules are necessary to avoid some semantical problems.

1. Auto triggers couple two different events along the trajectory: the one corresponding to the auto trigger itself, and the one corresponding to the trigger referenced by it, where the auto condition must be fulfilled. In the time interval between these events (auto interval) no conditions should be evaluated. Therefore, conditional branches and conditional loops should never appear inside the auto interval. Otherwise, the composite would describe an unpredictable aircraft behaviour, since the auto trigger could have multiple solutions.

In order to avoid this problem, auto intervals must be disjoint to execution intervals of conditional branches or loops. As an exception, the auto interval (i.e., the auto trigger and its referenced trigger) can be also completely enclosed inside the conditional branch or loop.

2. Composites in a conditional branch or conditional loop may be executed once, more than once or may not be executed at all. Thus, neither it is possible for linked or auto triggers outside a conditional branch or conditional loop composite to reference any trigger inside of it; nor it is possible for linked or auto triggers inside a conditional loop to refer to a trigger outside of it. Otherwise, the composite would describe an undefined behaviour, as some triggers reference events that might never happen, or happen multiple times.

Figure 7.5 depicts four composites that follow these rules. Details on this graphical representation are provided in section 7.2 , but for now it is sufficient

\footnotetext{
${ }^{3}$ The configuration vector can only be used when defining conditions for fixed-wing aircraft that have aerodynamic configuration (landing gear, high lift and speed brakes).

${ }^{4}$ The environmental model vector contains information related to the weather (wind, temperature and pressure) and magnetic conditions.
} 


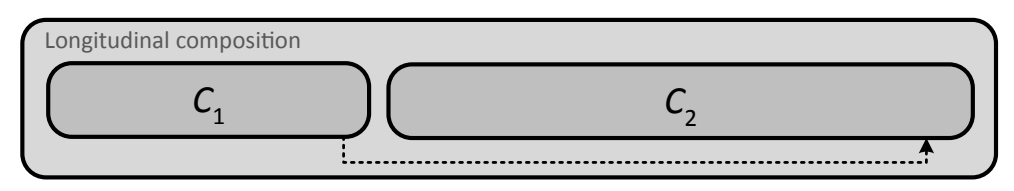

(a)

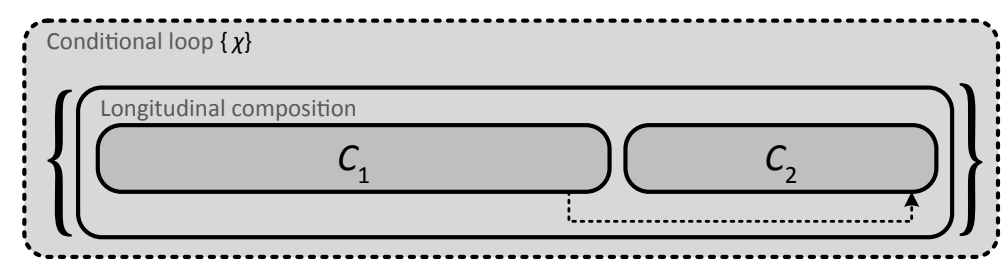

(b)

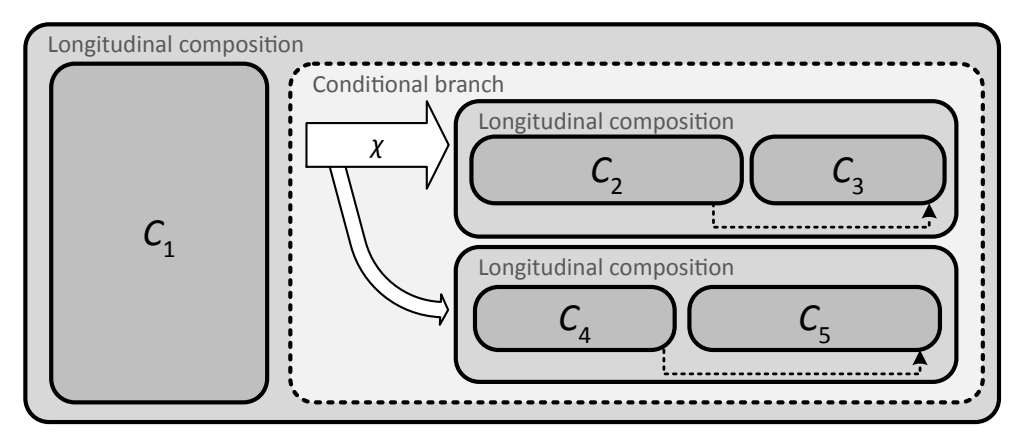

(c)

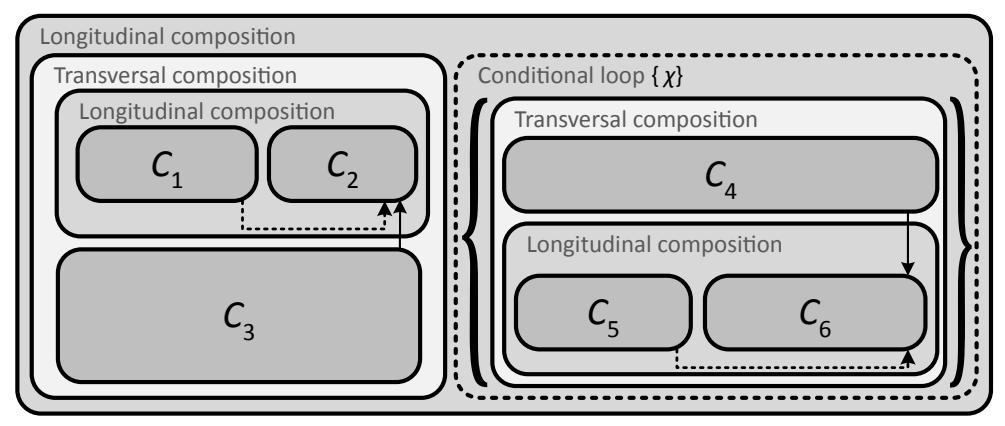

(d)

Figure 7.5: Valid composite operations 


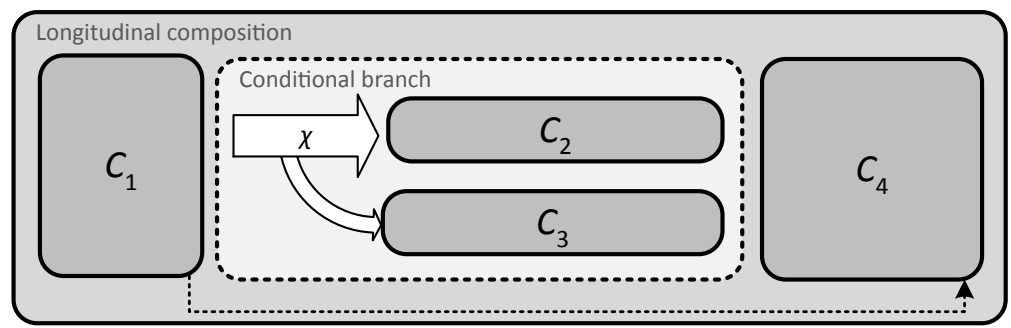

(a)

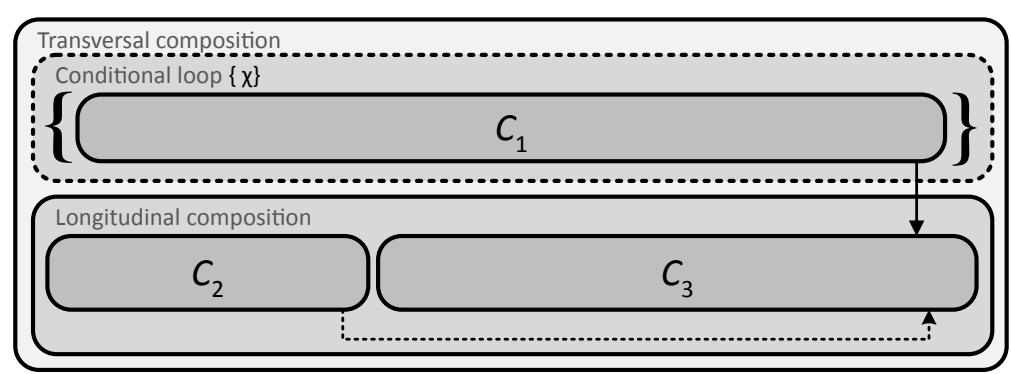

(b)

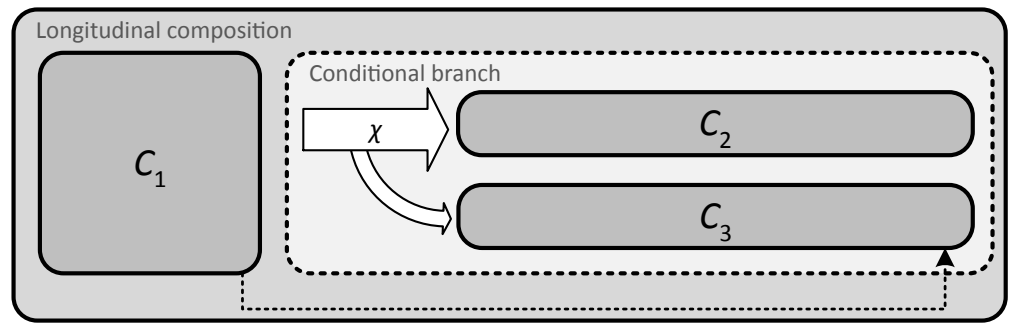

(c)

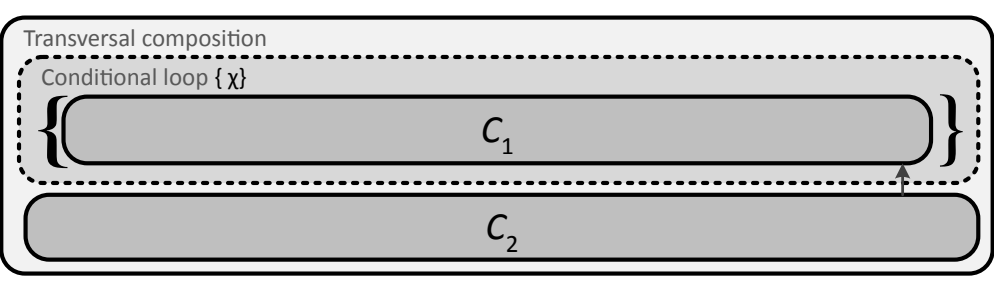

(d)

Figure 7.6: Invalid composite operations 


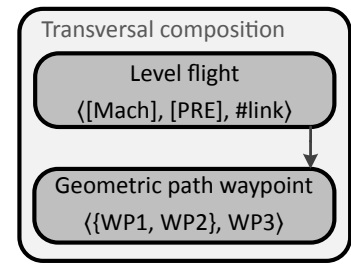

(a) Linked trigger.

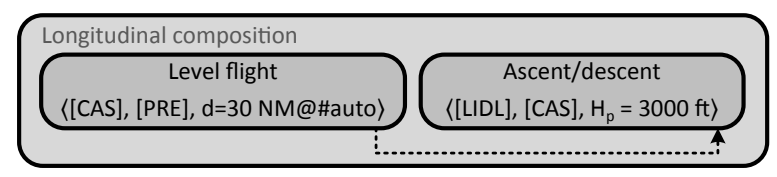

(b) Auto trigger.

Figure 7.7: Use of arrows for indicating referenced triggers.

to know that boxes represent composites, and arrows represent auto triggers (dashed lines) or linked triggers (continuous lines) pointing to the referenced trigger. Composite in figure 7.5a does not have any conditional branches or loops. In composites in figures $7.5 \mathrm{~b}$ and $7.5 \mathrm{c}$, the auto intervals of the auto triggers and the execution intervals of conditional branches and loops overlap; but since the former are completely enclosed in the latter, the operations are correct. In figure $7.5 \mathrm{~d}$, one of the auto intervals is disjoint to the execution interval of the conditional loop, whereas the other auto interval is completely enclosed.

Some composites that do not follow these rules are shown in figure 7.6. In figures 7.6a, 7.6b, and 7.6c, auto triggers overlap with the execution interval of a conditional loop or branch, and the former are not completely contained in the latter. In figures 7.6c and 7.6d, there are triggers referencing another trigger in a conditional branch or loop from outside of it.

\subsection{Graphical representation}

A graphical notation for the representation of composites has been informally introduced along this chapter. This section is devoted to provide a more detailed description of this notation.

Elementary composites are depicted as gray boxes containing the name of the template and the values of its parameters, following the notation of appendix D with one exception: linked and auto triggers don't use tag names to referenced triggers; arrows are used instead. In the textual representation of elementary composites, tag names are replaced by a fixed keyword (\#link for linked triggers, \#auto for auto triggers). Referenced triggers are indicated with an arrow starting at the elementary composite containing the linked or auto trigger, and pointing to the elementary composite containing the referenced trigger. Continuous lines are used for linked triggers, and dashed lines are used for auto triggers. An example can be found in figure 7.7.

Composites resulting from operations are represented as boxes containing the operands. Each operand has a characteristic design, as shown in figure 7.4. Conditions are expressed using the notation of appendix D. Note that, as the longitudinal composition is an associative operation, it is possible to include more than two operands in the same operation. The same applies to the transversal composition. 


\subsection{Examples}

In this section, a series of examples are used to illustrate the main features of the ICDL.

\subsubsection{Commercial flight}

The first example intends to show the simplicity of defining a flight using ICDL when compared to defining the same flight using AIDL. In ICDL, a flight can be built from a few elementary composites. For example, the composite in figure 7.8 describes a fragment of a simple commercial flight. In the composite, the vertical, lateral, and configuration profiles are completely decoupled. This composite assumes some initial conditions, where the aircraft is already at 3000 feet and with a CAS of 240 knots. At the end of the composite, the aircraft will have the same altitude and speed, at a point near the destination airport.

The vertical profile includes the three phases of flight: climb, cruise, and descent. The aircraft will climb and accelerate using an energy share factor (ESF) of 0.5 until it reaches the ascent speed of 280 knots, and then it will perform a CAS/Mach ascent. The cruise phase is represented with a single level flight, with the top of descent defined implicitly using an auto trigger. This definition states that the top of descent must be wherever necessary so that the aircraft will be at exactly 3000 feet at the end of the composite. The descent phase is defined using a Mach/CAS descent followed with a deceleration with a path angle of -1.5 degrees.

The lateral profile is specified using a single composite that indicates a sequence of waypoints. In this case, the sequence of waypoints corresponds to a flight between Seattle-Tacoma International Airport (KSEA) and Los Angeles International Airport (KLAX). A fly-by is performed at each waypoint, using a default tolerance for all waypoints. Finally, the configuration profile is defined as clean during the whole flight.

The composite in figure 7.8 doesn't leave any DoFs open, and therefore it can be univocally translated to an AIDL word. The aircraft intent equivalent to this composite is shown in figure 7.9. In comparison, the aircraft intent would have been more difficult to generate by a human operator, and also more difficult to understand.

\subsubsection{Quadrotor flight}

A second example is included to show the additional semantics of the ICDL. Conditional branches and conditional loops are used in this example to describe a trajectory that cannot be described in the AIDL. In this case, the flight of a quadrotor aircraft in a surveillance mission is considered, so the templates used in this example correspond to those designed for quadrotors.

The objective of this flight is to watch two static targets at waypoints TGT1 and TGT2. The quadrotor will travel between the areas surrounding each target periodically. While in each area, it will fly in circles around the nearest target. The composite in figure 7.10 reflects this behaviour: The quadrotor will first fly to a point in the area of TGT1 (this point is called PT1). Then, it will fly in 


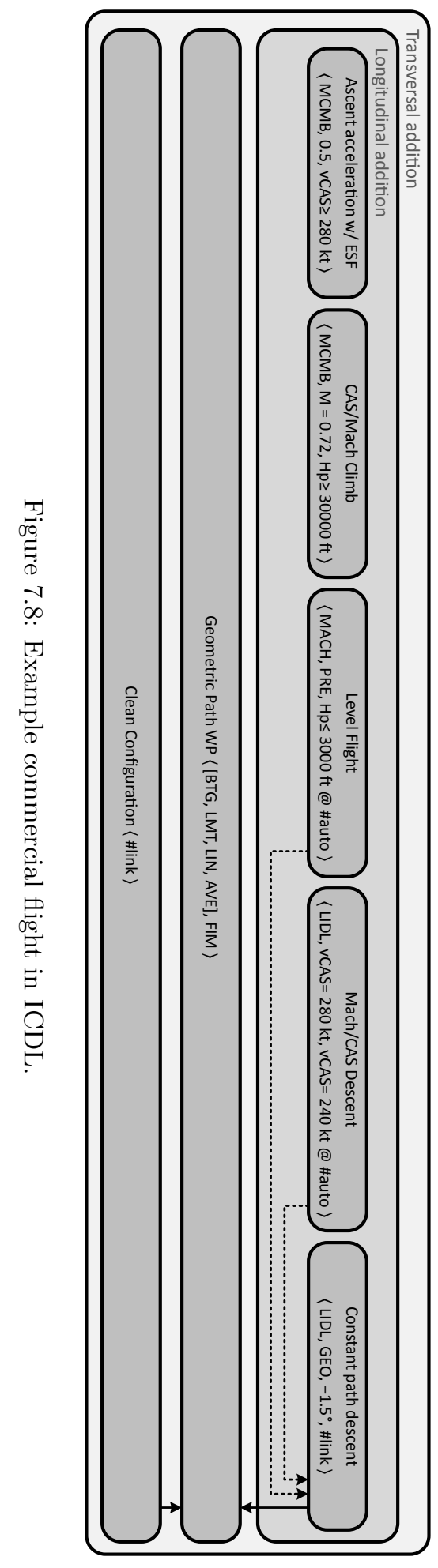




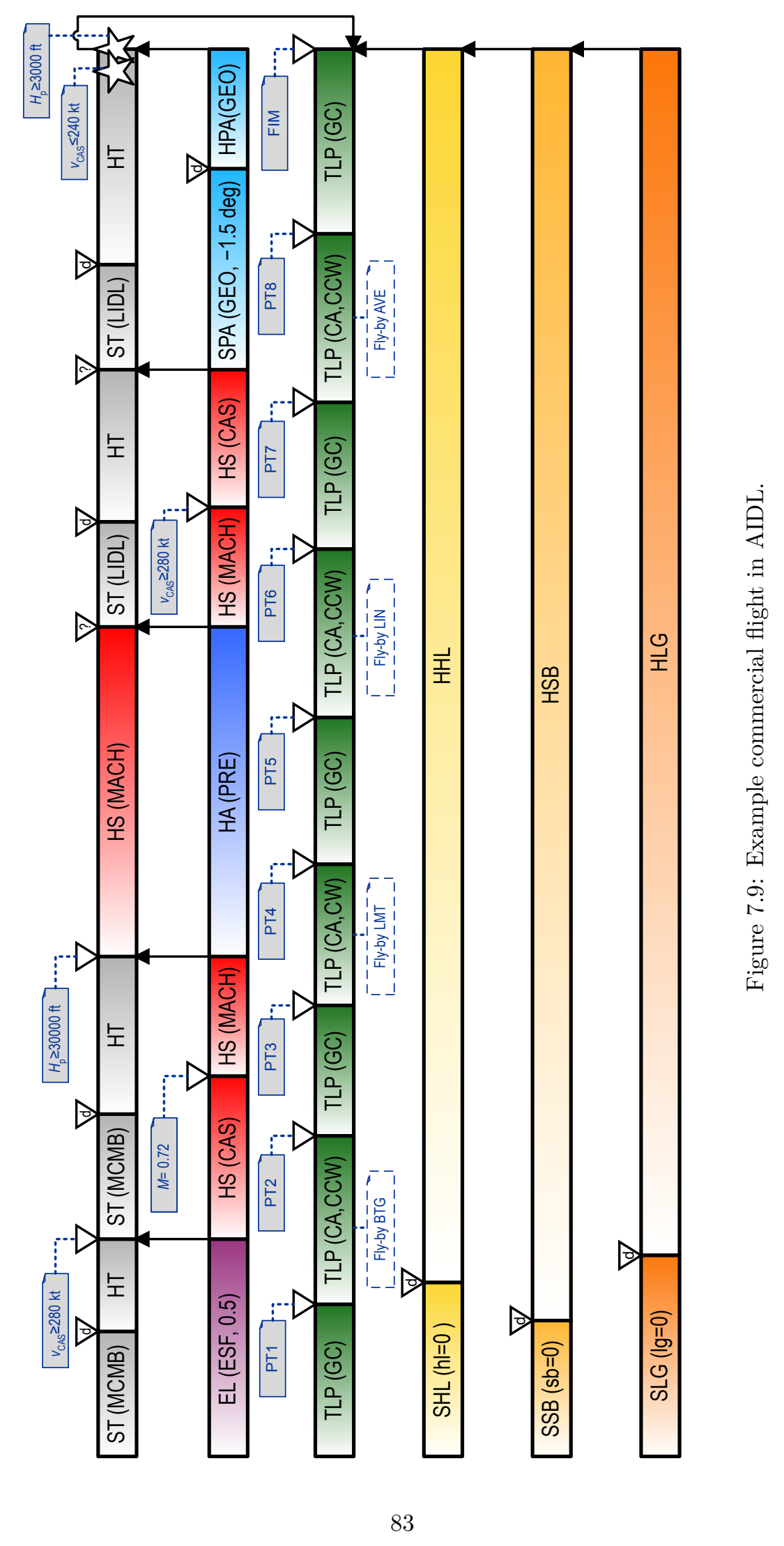


circles around TGT1, slowly descending to get closer to the target. After 30 seconds, the quadrotor must travel to PT2 in order to watch the other target. In this case, there is an obstacle in the way and the aircraft must have an altitude of at least 30 metres. If the aircraft is above that altitude before travelling to PT2, no additional manoeuvre is necessary. Otherwise, the aircraft should gain altitude before going to PT2. A conditional branch is used to achieve this conditional behaviour. Once in the area of TGT2, the quadrotor will also fly in circles around this location while descending. This process is repeated for 10 minutes, using a conditional loop.

This ICDL word doesn't have a unique translation to AIDL. The most obvious reason is that it doesn't cover all the DoFs, as no information regarding the yaw is provided. However, there are other reasons, related to the additional semantics in the ICDL, why this composite cannot be univocally translated into AIDL: The result of evaluating the conditions, and therefore the behaviour of the aircraft, cannot be determined before execution time. For example, the wind along the $z_{e}$ axis $\left(w_{z}\right)$ may determine whether the condition in the conditional branch is met or not. The same occurs with the condition in the conditional loop. The number of times the quadrotor will visit both targets depends on the distance between both targets (which can be computed in advance), but also on the wind and on which composite in the conditional branch is executed.

\subsection{Final words}

In this chapter, a different language for describing aircraft trajectories has been defined. The development of this language, the ICDL, has been performed as a collaboration between BR\&TE and the UPM.

This language arises to fix some of the limitations of the AIDL and provide some new features. These features enable some new applications of intent-based languages. For example, as the ICDL does not require all DoFs to be defined, it allows the generation of intent in several stages: in ATM trajectory prediction, different components may build the lateral, vertical, and configuration profiles separately. These three separated profiles can be represented as composites, which can then be combined (using transversal composition) to form the final intent describing the whole flight.

The use of templates also eases the process of generating a valid description of the trajectory. Templates define common manoeuvres, where some parameters are missing. This is analogous to the phraseology used for clearances, e.g., the clearance 'Climb to flight level 200' defines a climb manoeuvre and a necessary parameter, the target flight level. Many other clearances can be defined using templates, which make ICDL composites easier to build and understand.

Interval and set parameters allow defining multiple possible values for a given magnitude or specifier. However, since the aircraft can only fly using one of these values, particular magnitudes and specifiers must be selected among the different possibilities at some point. Rather than selecting values randomly, users want to select a combination of values that offers some advantage. In other words, the interval and set parameters can be used to optimize some magnitudes and specifiers of a flight, according to some criteria. In the next chapter, means 


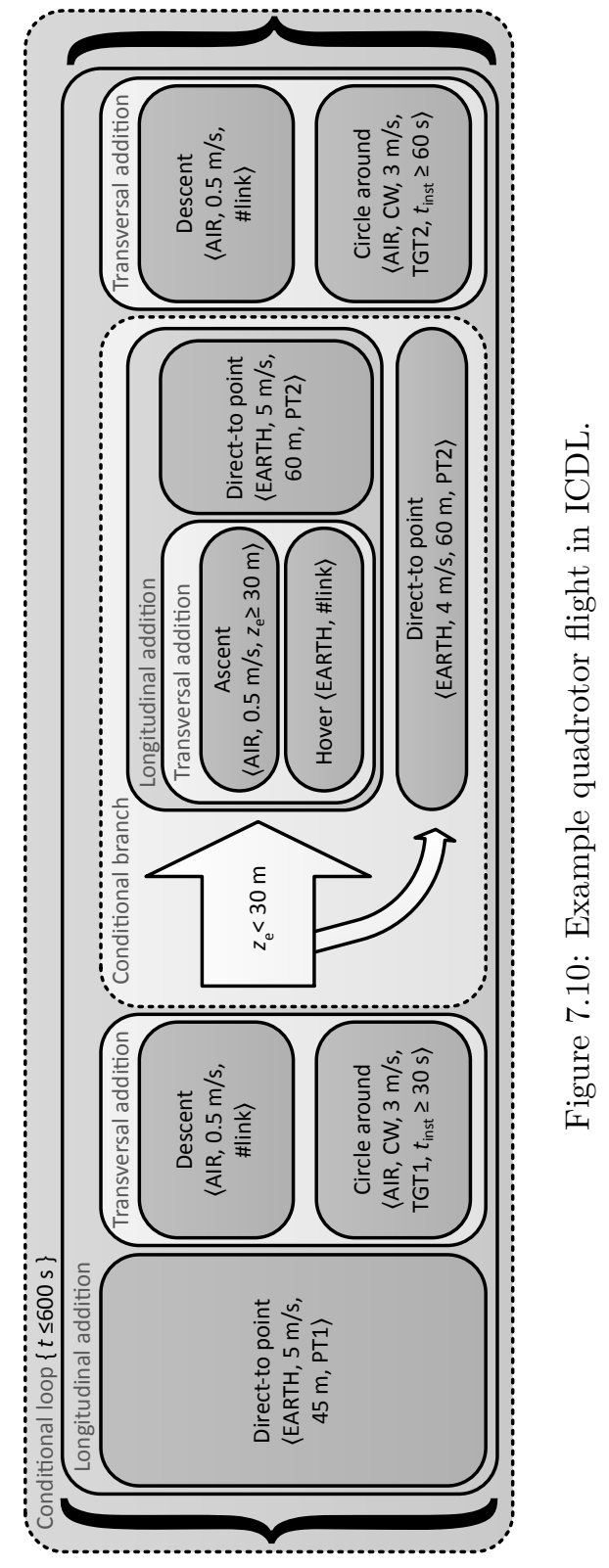


to specify these criteria (denoted objectives) are provided.

Despite the new features introduced in this language, which enable new applications, sometimes the flexibility provided may not be sufficient. For example, the number of defined DoFs in a composite must be constant. That is, if a composite defines some behaviour of the aircraft regarding one thread, that thread must be defined throughout the whole flight. An additional level of flexibility can be provided by adding another abstraction layer, in the form of a language that builds on top of the ICDL. This language is described in the next chapter. 


\section{Chapter 8}

\section{Flight intent description language}

The flight intent description language (FIDL) $[53,128]$ is a formal language used to describe flight intent. The flight intent (as discussed in section 2.3) contains the key operational requirements and constraints of the flight. It can be seen as a generalization of the flight plan information, including strategic or tactical amendments. This language, which was initially conceived by BR\&TE and then developed in collaboration with the UPM, is also included in the language hierarchy presented in chapter 5 .

Formally, the FIDL is an extension of the ICDL that provides the following features:

- In the FIDL, the flight is segmented in multiple stages — not necessarily matching the flight phases-, called flight segments. Each flight segment describes the behaviour of the aircraft during that stage. This description is provided as a composite expressed in ICDL.

A composite covers zero or more AIDL threads (i.e., closes zero or more DoFs), and the same threads are covered throughout the execution interval of the composite. In FIDL, different flight segments can cover different AIDL threads, and therefore they can be described with varying levels of detail.

- Flight segments are usually arranged as a linear sequence of elements, sorted chronologically. Additionally, the semantics for non-linear execution available in ICDL (conditional branches and conditional loops) are available at flight segment level.

- Flight information is not exclusively contained in flight segments. In FIDL it is possible to specify constraints that affect the open DoFs of the flight segments.

- The DoFs that have not been closed by the flight segments or constraints, can be closed by specifying objectives. Objectives represent desirable optimization targets of the flight. 
As FIDL is an extension of ICDL, it is also an extension of AIDL. That means that any intent that can be expressed in either AIDL or ICDL can also be expressed in FIDL. However, the opposite is not always possible. For example, any flight intent expressed in FIDL that contains any constraints or objectives cannot be univocally expressed in ICDL or AIDL.

\subsection{Language description}

An instance of FIDL contains three elements: an effect, a set of constraints, and a set of objectives. The effect is specified using flight segments, which are combined to form a single flight segment aggregation.

A notation for FIDL is outlined in the following sections, and a full specification of this notation is included in appendix D. In this notation, a flight intent expressed in FIDL is written as:

\section{FLIGHT SEGMENTS(aggregation) \\ CONSTRAINTS (constraint-set) \\ OBJECTIVES(objective-set)}

where aggregation is the effect of the flight intent (expressed as a flight segment aggregation), constraint-set is the set of constraints, and objective-set is the set of objectives. The remainder of this section describes these elements in detail.

\subsubsection{Flight segments}

Flight segments represent the intent of changing the aircraft dynamic state to reach a final state condition. This condition is indicated by the end trigger of the flight segment. The information on how the aircraft is operated until the condition is reached is provided using an ICDL composite. The end trigger of the flight segment corresponds to the end trigger of this composite. Therefore, a flight segment is completely characterized by a composite.

The information contained in this composite may range from a fully unknown manoeuvre (described using a 'Null' composite), to a complete description of how the aircraft is to be operated (using a composite specifying all AIDL threads $\left.^{1}\right)$.

The FIDL imposes some requirements to the composites that characterize flight segments. Since end triggers represent the desired final state of the aircraft, the end trigger of these composites must be either fixed, floating, waypoint, or list triggers. Auto, linked, and default triggers cannot represent aircraft state information themselves, and therefore are not admitted.

Given that a flight segment is completely characterized by a composite, flight segments use the same notation as composites.

\footnotetext{
${ }^{1}$ In order for the composite to be unambiguous, it should also have all the interval parameters closed, and all the set parameters should contain exactly one element.
} 


\subsubsection{Aggregations of flight segments}

The effect of a flight intent expressed in FIDL is constituted by an aggregation of flight segments. Most commonly, aggregations consist of a sequence of flight segments in chronological order. However, it some occasions it is desirable to specify a behaviour that depends on some conditions, and the FIDL provides the means to do so (with operations analogous to the conditional branches and loops from the ICDL). The rules to build flight segment aggregations are described below:

1. Any flight segment is a valid aggregation.

2. If $F_{1}$ and $F_{2}$ are valid aggregations, then the sequence aggregation of $F_{1}$ and $F_{2}$, denoted $F_{1} ; F_{2}$, is a valid aggregation. This indicates that $F_{2}$ is executed immediately after $F_{1}$.

3. If $F_{1}$ and $F_{2}$ are valid aggregations, then the branch aggregation of $F_{1}$ and $F_{2}$ with a condition $\chi$, denoted $\left\{F_{1} \mid F_{2}\right\}[\chi]$, is a valid aggregation. The condition $\chi$ is a mathematical expression as described in section 7.1.4. This indicates that, depending on how the $\chi$ evaluates, either the aggregation $F_{1}$ or $F_{2}$ is executed.

4. If $F_{1}$ is a valid aggregation, then the loop aggregation of $F_{1}$ with condition $\chi$, and two integers $m$ and $M$ (with $0 \leq m \leq M$ ), denoted $\left\{F_{1}\right\}[\chi: m: M]$, is a valid aggregation. The condition $\chi$ is a mathematical expression as described in section 7.1.4. This indicates that the aggregation $F_{1}$ is executed repeatedly, at least $m$ times and a maximum of $M$ times, while the condition $\chi$ is met. The integers $m$ and $M$ can be omitted (values $m=0$ and $M=\infty$ are assumed).

\subsubsection{Constraints}

A constraint represents a restriction on the dynamic states of the trajectory to be met. Constraints are specified by their domain of application (DoA), effect, and priority.

A flight intent expressed in FIDL may contain any number of constraints. However, in order to be semantically correct, all constraints must be compatible. Priorities in constraints help dealing with contradictory constraints in some cases. However, if two constraints with the maximum priority specify incompatible restrictions (e.g., require the aircraft to be above 6000 feet and below 4000 feet at the same time), that flight intent would be semantically incorrect.

\section{Domain of application}

The set of aircraft states where the constraint is active is the DoA of the constraint. DoAs can be specified as a general mathematical expression of the type:

$$
f(\mathbf{X}, \mathbf{u}, \boldsymbol{\Delta}, \mathbf{E}, t) \leq 0
$$

where $f$ is a function of the aircraft state vector $\mathbf{X}$, the control vector $\mathbf{u}$, the configuration vector $\boldsymbol{\Delta}$, the environmental model vector $\mathbf{E}$, and the time $t$. Thus, 
a DoA may not only be a geometrical area or volume, but a hypervolume defined by any combination of the aforementioned variables. However, in ATM and UAS applications, the DoA corresponds typically to geometric areas or volumes, such as points, segments or airspace volumes.

Additionally, DoAs contain a specifier that indicates whether the constraint is active in every state where the expression is met (called a strong DoA), or just in at least one of these states (called a weak DoAs). In the notation used here, weak DoAs are prefixed by ${ }^{(\mathrm{w})}$, while strong DoAs, much more prevalent, do not have any associated notation.

This notation also provides simplified means to denote DoAs corresponding to points, segments, or airspace volumes. These DoAs can be simply described as a point or list of points, with an optional range of altitudes. A DoA that encompasses the whole flight is called a universal DoA and it's denoted $\Omega$.

Some examples of DoAs are shown below:

- FAYTA: a strong DoA defined by named point from AIP Spain [146].

- $\{$ CANIS, ENETA $\}$ : a strong DoA defined as a segment between two points from the same AIP.

- (w) $\{$ PT1, PT2, PT3, PT4 $\}[0 \mathrm{ft}, 8000 \mathrm{ft}]$ : a weak DoA with four points defining the perimeter of a volume, and its altitude boundaries.

- $v_{\text {TAS }} \leq 280 \mathrm{kt}:$ a strong DoA defined as a range of speeds. This expression is equivalent to $(8.1)$ where $f(\mathbf{X}, \mathbf{u}, \boldsymbol{\Delta}, \mathbf{E}, t)=v_{\mathrm{TAS}}-280 \mathrm{kt}$. For convenience, simplified expressions such as the one above are also allowed.

\section{Effect}

The constraint effect is the condition to be satisfied in the DoA. It is a mathematical expression of the form:

$$
g_{1}(\mathbf{X}, \mathbf{E}, t) \leq f(\mathbf{X}, \mathbf{u}, \boldsymbol{\Delta}, \mathbf{E}, t) \leq g_{2}(\mathbf{X}, \mathbf{E}, t)
$$

where $f$ is a function of $\mathbf{X}, \mathbf{u}, \boldsymbol{\Delta}, \mathbf{E}$, and $t$; and $g_{1}$ and $g_{2}$ are functions of $\mathbf{X}, \mathbf{E}$, and $t$. This expression can be simplified in some cases for the sake of simplicity. If one of the boundaries, $g_{1}$ or $g_{2}$, is not necessary (i.e., $g_{1}=-\infty$ or $g_{2}=\infty$ ), then the corresponding inequation can be omitted. For example, it is possible to write $f \leq g_{2}$ instead of $-\infty \leq f \leq g_{2}$. If $g_{1}=g_{2}$, expression (8.2) can be written as $f=g_{1}$.

As with DoAs, simplified constraint effect descriptions are available for specific constraint types. In particular, if the aircraft is required to follow an airway, standard instrument departure route (SID), standard arrival route (STAR) or leg between two points (i.e., in a segment DoA), the constraint effect can be defined by indicating the name of said airspace structure. These types of effect impose a restriction on the horizontal path, which must follow the specified airspace structure (with some threshold).

Some examples of valid effects are:

- $H_{p} \leq 10000 \mathrm{ft}$ : geopotential pressure altitude at or below 10000 feet. 
- $0.65 \leq M \leq 0.80$ : speed between 0.65 and 0.80 Mach.

- TERTO3C: a named SID from AIP Spain [146].

\section{Priority}

Constraints have a priority, specified as a real number $P(0 \leq P \leq 1)$, that indicates how relevant a constraint is (relative to other constraints in the same flight intent). A priority $P<1$ indicates that a constraint can be discarded if it is incompatible with another constraint with higher priority. Mandatory constraints $P=1$ cannot be discarded under any circumstances. Priority is denoted as a number in parenthesis before the constraint's effect. If $P=1$, it is omitted.

For example, airlines can specify preferred climb/descent speeds as low priority constraints. If the ATCO issues a mandatory constraint specifying a different climb/descent speed, the former constraint could be discarded.

\section{Examples}

Complete examples of constraints are presented below. These examples use the notation of appendix D, where constraints are expressed as an effect, followed by an 'at' sign (@), followed by the DoA. This is prefixed by the priority, if the constraint is not mandatory.

- $12000 \mathrm{ft} \leq h @$ FAYTA: at FAYTA (see [146]), geometric altitude $(h)$ must be at or above 12000 feet.

- $140 \mathrm{kt} \leq v_{\text {TAS }} \leq 190 \mathrm{kt} @ h \leq 6000 \mathrm{ft}:$ TAS between 140 and 190 knots, if geometric altitude is below 6000 feet.

- $H_{p} \geq 32000 \mathrm{ft} @{ }^{(w)} \Omega$ : geopotential pressure altitude $\left(H_{p}\right)$ at or above 32000 feet at least once during the whole flight (a weak DoA is used).

- $(0.5) v_{\mathrm{CAS}}=250 \mathrm{kt} @ \mathrm{~d} H_{p} \leq-1000 \mathrm{fpm}$ : constant CAS of 250 knots if descending at a rate of 1000 feet per minute or faster, with a priority of 0.5 .

- TERTO6C @ \{TERTO,ENETA\}: follow a STAR named TERTO6C between waypoints TERTO and ENETA.

\subsubsection{Objectives}

Objectives represent a desire to maximize or minimize a functional on the aircraft trajectory. Like constraints, objectives have an effect and a DoA. They also have a weight that represents the relative effect of one objective with respect to others in the same flight intent.

Objective DoAs are analogous to constraint DoAs, except that there are no weak objective DoAs. The effect of an objective is a mathematical function describing the impact of the aircraft motion on the specified cost per time unit, $f(\mathbf{X}, \mathbf{u}, \mathbf{E}, t)$. The complete cost of an aircraft trajectory related to an objective 
can be calculated by integrating this function over the objective's DoA, denoted $\Phi$.

$$
\int_{\Phi} f(\mathbf{X}, \mathbf{u}, \mathbf{E}, t) \mathrm{d} t
$$

The optimal aircraft trajectory with respect to a certain objective will be the one that minimizes (8.3). One example of a valid objective is $1 @ \Omega$, which aims to minimize total flight time, as the result of integrating 1 over time is the total time of the flight. Another possible objective could be $-\mathrm{d} m_{f} / \mathrm{d} t @ \Omega$, which minimizes the fuel consumption, provided $\mathrm{d} m_{f} / \mathrm{d} t$ measures the rate of fuel consumption.

When multiple objectives are to be considered, the cost function to be minimize is a weighted sum including every objective:

$$
w_{1} \int_{\Phi_{1}} f_{1}(\mathbf{X}, \mathbf{u}, \mathbf{E}, t) \mathrm{d} t+\ldots+w_{n} \int_{\Phi_{n}} f_{n}(\mathbf{X}, \mathbf{u}, \mathbf{E}, t) \mathrm{d} t
$$

where $w_{i}, \Phi_{i}$, and $f_{i}$ are the weight, DoA, and effect of the $i$-th objective $(1 \leq$ $i \leq n)$, respectively. In the notation in appendix $\mathrm{D}$, each objective is denoted $\left(w_{i}\right) f_{i} @ \Phi_{i}$.

Many commercial airlines use the cost index (CI) to express the total cost of operating the aircraft on a given flight $[61,86]$. In those cases, the operating cost is computed using the following equation:

$$
\text { cost }=\Delta m_{f}+\mathrm{CI} \times \Delta t
$$

where $\Delta m_{f}=\int_{\Omega}-\mathrm{d} m_{f}$ is the decrease of fuel mass, and $\Delta t=\int_{\Omega} \mathrm{d} t$ is the flight time. Equivalently, the desire to minimize this cost can be expressed using two objectives. Since the CI is the ratio of time-related costs to fuel cost, minimizing (8.6) is equivalent to minimizing (8.5) according to some CI.

$$
\int_{\Omega}-\frac{\mathrm{d} m_{f}}{\mathrm{~d} t} \mathrm{~d} t+\mathrm{CI} \int_{\Omega} \mathrm{d} t
$$

The two objectives can be denoted (1) $-\mathrm{d} m_{f} / \mathrm{d} t @ \Omega$ and (CI) $1 @ \Omega$.

\subsection{Graphical representation}

A graphical notation for describing flight segment aggregations is described in this section. This notation is based on the notation for ICDL described in section 7.2. No graphical notation is included for constraints or objectives, for which the notation in appendix $\mathrm{D}$ can be used.

A flight segment is represented as a white box containing a composite. Flight segments in a sequence aggregation are displayed side by side as shown in figure 8.1a. Branch and loop aggregations are shown in figures $8.1 \mathrm{~b}$ and $8.1 \mathrm{c}$, respectively. 


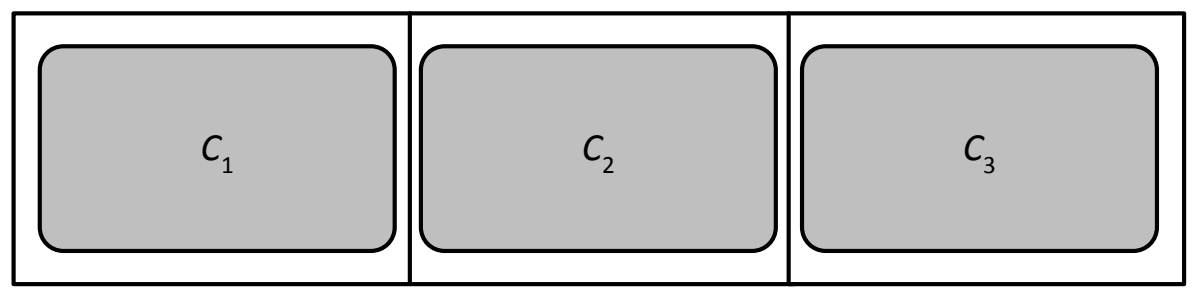

(a) Sequence aggregation.

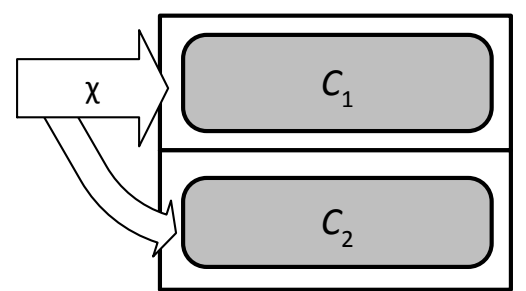

(b) Branch aggregation.

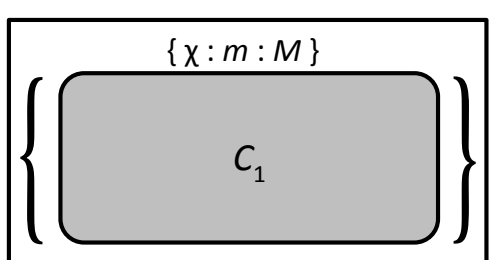

(c) Loop aggregation.

Figure 8.1: Graphical representation of flight segment aggregations.

\subsection{Examples}

The examples in this section illustrate the features of the FIDL, including scenarios that cover several different contexts.

\subsubsection{Commercial flight}

One of the simplest possible flight intents expressed in the FIDL would contain no constraints or objectives, and only one flight segment. This flight segment would only specify the destination of the flight, which could be the destination airport in a commercial flight. This flight intent can be written as:

\section{FLIGHT SEGMENTS $(\langle$ GCPL $\rangle)$}

Where GCPT is the ICAO code for the Gran Canaria Airport. The flight intent above can also be described using the graphical notation, as shown in figure 8.2 .

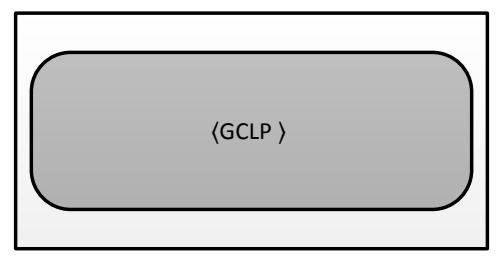

Figure 8.2: Minimum FIDL example.

Of course, the example above can be expanded to include more relevant information. The following flight intent includes more intermediate points, constraints related to those points, and an objective affecting the whole flight. 


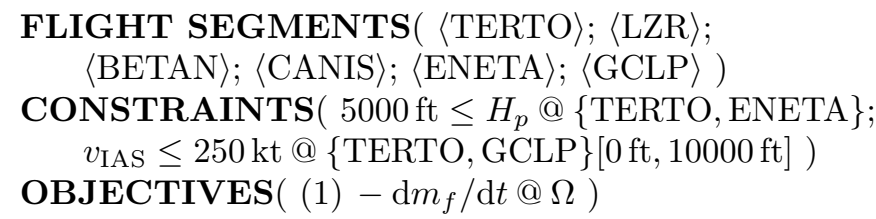

The intermediate points added correspond to one of the STARs to the destination airport. The two constraints are also associated to the terminal manoeuvring area (TMA) of this airport: one is the maximum altitude in the STAR, the other is the speed limit in the TMA. The objective in this flight intent minimizes the fuel burnt during the flight. More examples related to this scenario are shown in chapter 11 .

\subsubsection{Firefight flight}

The FIDL can also be used to describe other types of flight. Here, a firefighting mission is modelled, showing the versatility of the language. A firefight aircraft, the Bombardier 415 Superscooper airtanker, is assumed to be flying the modelled flight. This flight intent describes a flight with two main tasks: First, the aircraft has to reach the water source where the water is taken from (a lake near the fire), using a scooping manoeuvre. This is a precise operation that must be defined with high level of detail, using composites. Second, the water has to be transported to the location of the fire, and a water-dropping manoeuvre must be performed to release the water at the exact location. Again, a composite is used to detail this manoeuvre. The two main tasks described above are repeated until the fuel level reaches some threshold (about $20 \%$ of capacity). Finally, the aircraft must return to the base, the Greater Rochester International Airport (KROC), for refuelling.

This flight intent can be written as follows:

FLIGHT SEGMENTS $(\{\langle$ SCOOP_START $\rangle$;

$\{$ Constant path constant speed ascent/descent

$$
\langle[\mathrm{CAS}],[\mathrm{GEO}],-1.85 \mathrm{deg}, h=0 \mathrm{~m}\rangle
$$

$\oplus$ Level flight $\left\langle[\mathrm{CAS}],[\mathrm{GEO}], d_{\mathrm{inst}}=1350 \mathrm{ft}\right\rangle$

$\oplus$ Constant path ascent/descent

$\langle[\mathrm{MCMB}],[\mathrm{GEO}], 1.85 \mathrm{deg}, \# \mathrm{ma} 1\rangle\}$

$\otimes$ Geometric path waypoint $\langle\{\}$, SCOOP_END $\rangle \#$ ma1

$\otimes$ Clear configuration $\langle \#$ ma1 $\rangle$;

$\langle$ DROP_START $\rangle$;

Level flight $\langle[\mathrm{CAS}],[\mathrm{GEO}], \#$ ma2 $\rangle$

$\otimes$ Geometric path waypoint $\langle\{\}$, DROP_END $\rangle \#$ ma2

$\otimes$ Clear configuration $\langle \#$ ma2 $\rangle$;

\}$\left[m_{F} \geq 900 \mathrm{~kg}\right]$;

$\langle$ KROC $\rangle)$

CONSTRAINTS $(14 \mathrm{~m} \leq h \leq 15 \mathrm{~m} @$ SCOOP_START;

$70 \mathrm{kt} \leq v_{\mathrm{CAS}} \leq 75 \mathrm{kt} @ \mathrm{SCOOP} \_$START;

$150 \mathrm{~m} \leq h \leq 160 \mathrm{~m} @$ DROP_START;

$105 \mathrm{kt} \leq v_{\mathrm{CAS}} \leq 110 \mathrm{kt} @$ DROP_START $)$ 


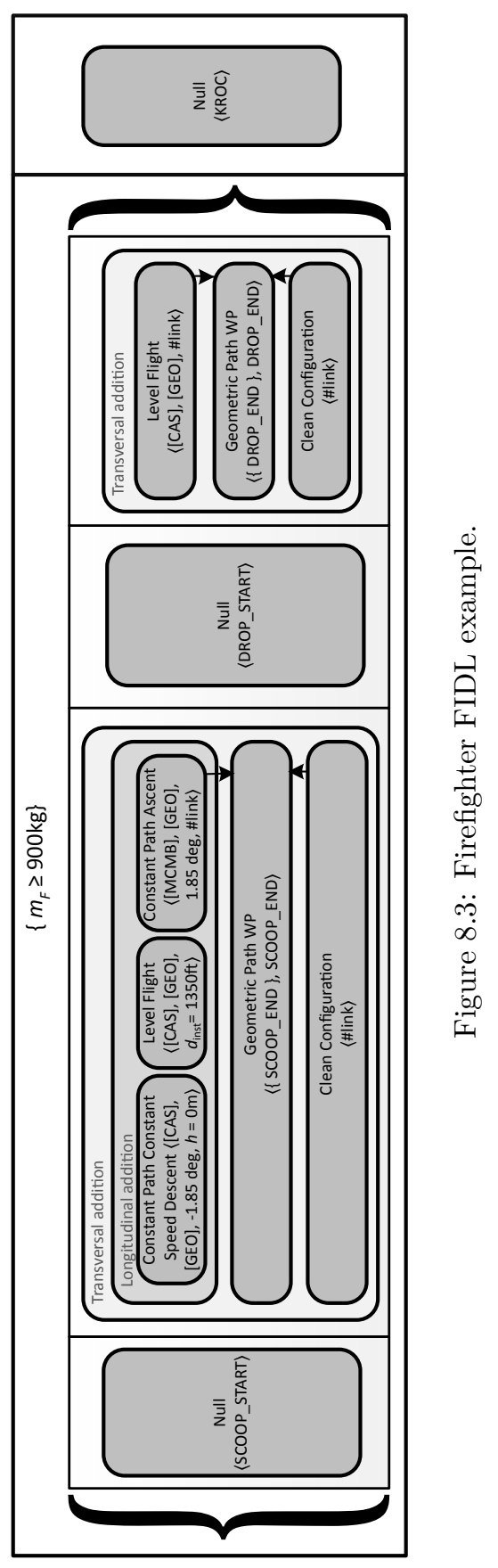




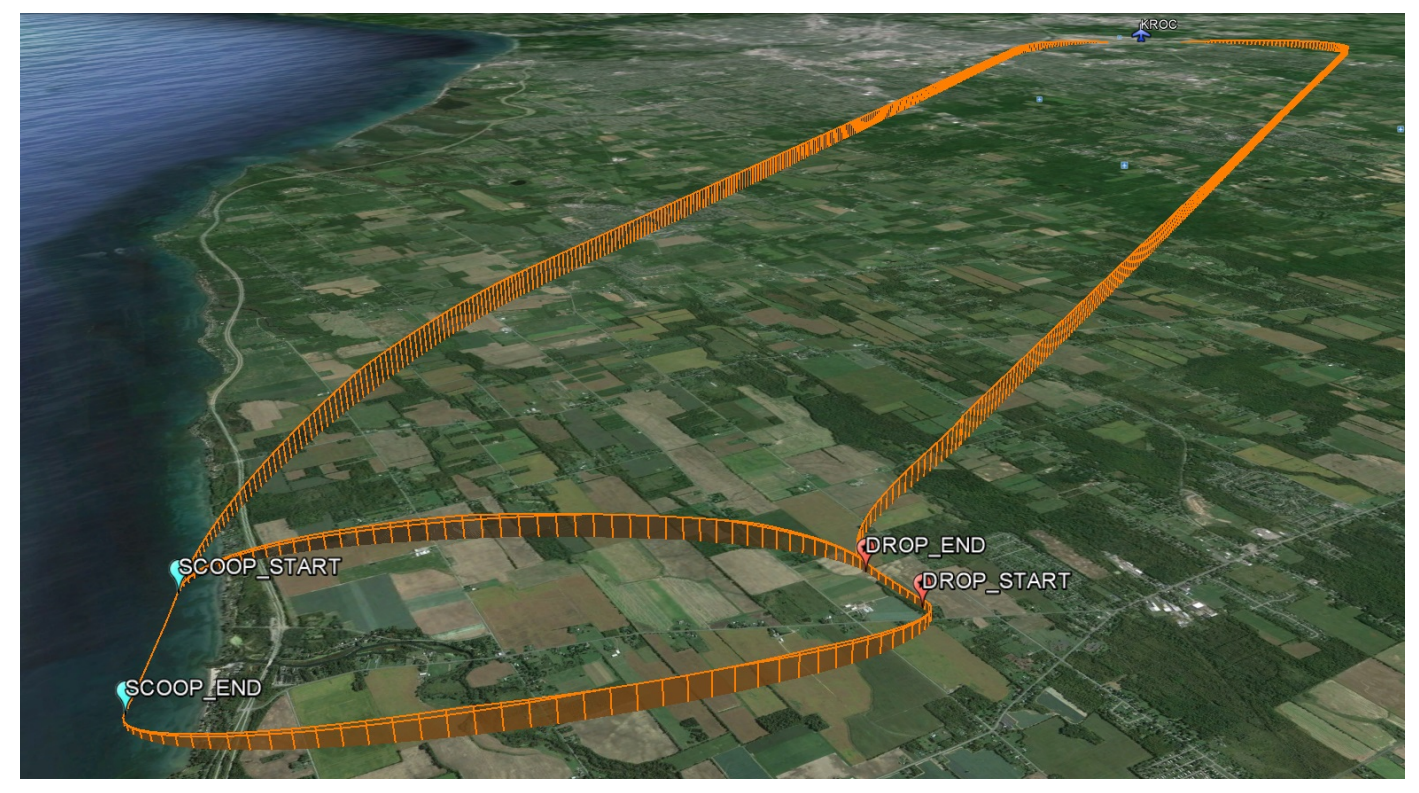

Figure 8.4: Firefighter example trajectory.

An equivalent flight intent (except for the constraints) using the graphical notation is shown in figure 8.3. The first element in the flight intent is a loop aggregation of four flight segments. The first flight segment commands the aircraft to go to the point where the scooping manoeuvre starts (the SCOOP_START point), with an specific altitude and speed (given as a constraint). The second flight intent contains a composite describing the manoeuvre in detail: the descent to the water level is performed using a specific path angle, and after filling the water tanks, an ascent to a safe altitude is performed. Then, the point where the water-dropping manoeuvre starts (DROP_START) must be reached. Again, constraints are used to ensure a particular altitude and speed at this point. Another composite completely describes this manoeuvre, which consists on a level flight over the fire. Finally, if the condition in the loop aggregation is met - the mass of fuel $m_{F}$ is at least 900 kilograms - , the aircraft will go back to the SCOOP_START point, and the process is repeated. Otherwise, the aircraft will go to the airport.

The coordinates of the points used in this flight intent are shown in table 8.1.

\begin{tabular}{l|l|l} 
Point name & Longitude & Latitude \\
\hline \hline SCOOP_START & $77.8792^{\circ} \mathrm{W}$ & $43.3528^{\circ} \mathrm{N}$ \\
SCOOP_END & $77.8946^{\circ} \mathrm{W}$ & $43.3572^{\circ} \mathrm{N}$ \\
DROP_START & $77.9129^{\circ} \mathrm{W}$ & $43.3166^{\circ} \mathrm{N}$ \\
DROP_END & $77.9059^{\circ} \mathrm{W}$ & $43.3169^{\circ} \mathrm{N}$ \\
KROC & $77.6793^{\circ} \mathrm{W}$ & $43.1169^{\circ} \mathrm{N}$
\end{tabular}

Table 8.1: Firefighting flight point coordinates

Assuming that the aircraft is initially at the KROC airport, the resulting 


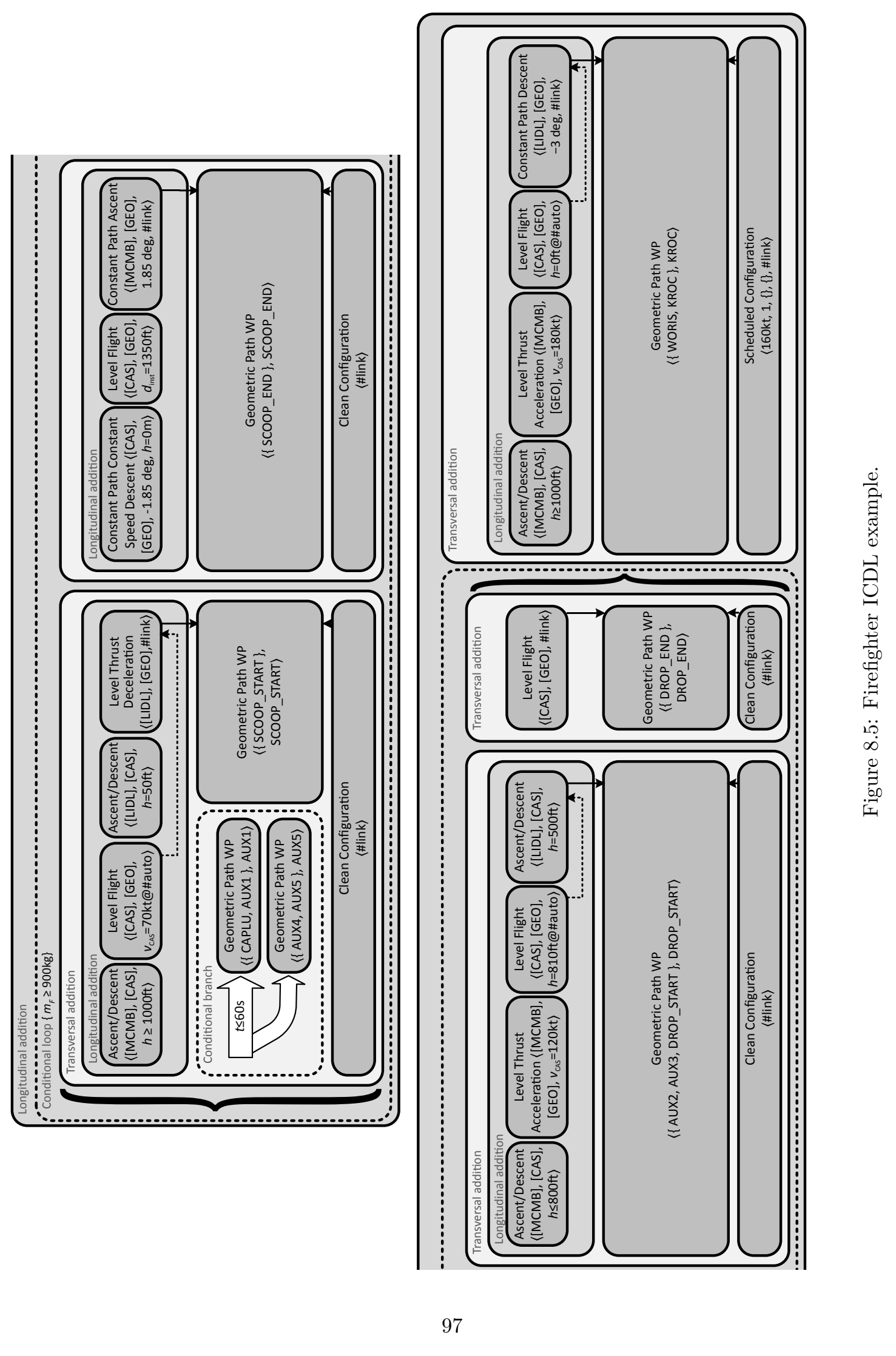




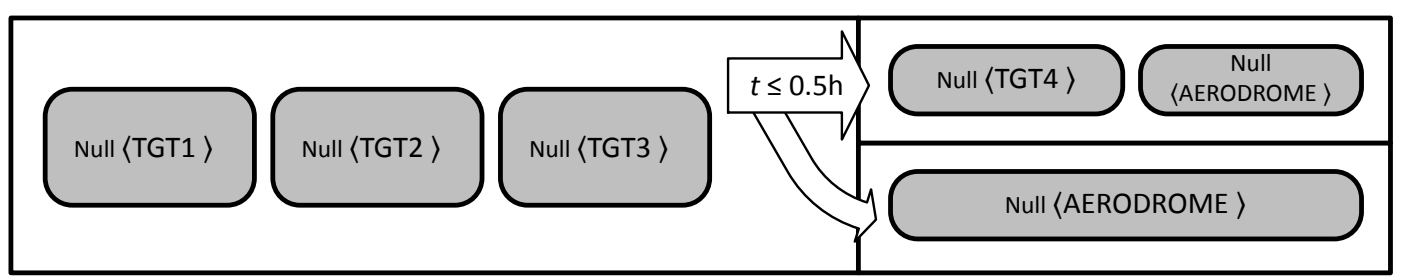

(a) UA specification.

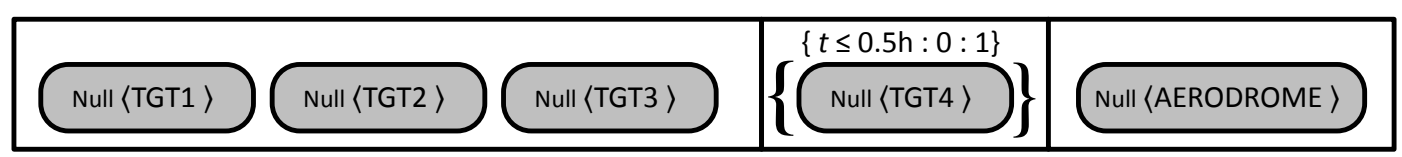

(b) Alternate UA specification.

Figure 8.6: UA FIDL examples.

trajectory from this flight intent is expected to be similar to the trajectory shown in figure 8.4. However, as this flight intent leaves all DoFs open during several phases of the flight, this trajectory could vary considerably. In most cases, trajectories aren't computed from a flight intent expressed in FIDL. Instead, the flight intent is previously translated to an aircraft intent or, equivalently, to a composite where all DoFs are closed. One possible translation of the flight intent described here to ICDL is shown in figure 8.5. This translation keeps all the composites intact, except the Null composites, which are replaced with other composites that completely specify the behaviour of the aircraft, taking into account the constraints in the flight intent and the operational context (i.e. departure and arrival procedures at the airport).

\subsubsection{UA reconnaissance mission}

Lastly, this example shows a tactical reconnaissance mission carried out by an UA, which may be a fixed-wing aircraft or a quadrotor. In this mission, the UA will photograph several static targets in a predefined time. Initially, three targets will be considered and, if the time invested in visiting them doesn't exceed 30 minutes, a fourth target is visited. Finally, the UA will return to the aerodrome.

There are several ways of expressing this behaviour in FIDL. One way is using the branch aggregation, as shown in figure 8.6a. After visiting the first three targets, the aircraft will follow a path that visits the fourth target before returning to de aerodrome, or one that returns to the aerodrome directly. Another way to express the same behaviour uses a loop aggregation, as shown in figure $8.6 \mathrm{~b}$. In this case, the path that goes to the fourth target is executed only if the time condition is met. Note that this loop is executed at most one time, so the aircraft won't visit the fourth target more than once. 


\subsection{Final words}

In this chapter, another layer of abstraction has been added on top of the ICDL, enabling the description of trajectories at a higher level. The FIDL provides new semantics that allow describing a trajectory based in what must be achieved, as opposed to previously defined languages that describe trajectories based on how the aircraft must behave. These new semantics are constraints and objectives. Constraints represent restrictions to the aircraft's trajectory, whereas objectives represent optimization goals.

As this language is built on top of the ICDL and the AIDL, trajectories described using the FIDL can provide an arbitrary level of detail. The FIDL can be used to describe flight intent, a description with a level of abstraction similar to what can be found in a flight plan; but it can also be used to provide the same level of detail as the AIDL or the ICDL. Moreover, a different level of detail may be used in each section of the flight. All this flexibility is possible due to the relationship between these languages.

This flexibility makes the FIDL an excellent candidate for providing flight information to a trajectory predictor. All the information regarding a flight can be expressed in FIDL regardless how detailed this information is. A trajectory predictor can then use the provided flight intent to generate an expected trajectory based on that information. Other applications of the FIDL could include filing a flight plan, negotiations in a CDM processes, or trajectory synchronization for other purposes.

In the hierarchy outlined in chapter 5 , the FIDL is the highest level language for describing trajectories of a single aircraft. In the next chapter, the FIDL will be further extended, enabling the specification of missions involving several aircraft. 



\section{Chapter 9}

\section{Mission intent description language}

In this chapter, the hierarchy of languages described in previous chapters is expanded to allow the description of missions. A new language, the mission intent description language (MIDL), is defined to provide the means to express mission intent. This language is built on top of the FIDL, inheriting all the expressive power of this language, and allowing to describe flights of more than one aircraft.

In the MIDL, actions carried out by different aircraft are organized in tasks. A task may describe the whole trajectory of one aircraft, from the start to the end of the mission; or describe a fragment of its trajectory that fulfils a specific goal. For example, a task may require one aircraft to go to a particular point or watch some area. The MIDL allows defining conditions specifying when the task must start (e.g., after another task is completed) and/or which aircraft must perform it (e.g., a camera-equipped aircraft). Note that these conditions may not unambiguously define which aircraft will perform each task, or when tasks will start. In some cases, it will be of interest to leave that information unspecified (at least in part), so it can be later decided by a mission planner or an operator.

Tasks may also represent recurring goals with a similar specification, save for some parameters. The MIDL also provides task templating mechanisms, so that the writing of the FIDL word describing a particular task can be written only once, or even delegated to a computer algorithm.

In a mission, different types of aircraft may be available. In this chapter, it will be assumed that available vehicles are either fixed-wing or quadrotor aircraft. It has been shown that the FIDL can be used to describe trajectories of these two types of aircraft, and in the MIDL both types can be combined.

The remainder of this chapter describes the details of the MIDL and provides several examples to showcase its potential applications. 


\subsection{Language description}

As stated before, the main elements that form a mission intent are the tasks. Tasks represent the ways that the mission goals must be achieved. Aircraft's behaviour during tasks can be specified using FIDL, but templates can also be used to abstract this behaviour and obtain simpler specifications with the same (or even more) expressive power. Since tasks, in general, leave some aspects of the mission or the aircraft's behaviour unspecified, constraints and objectives can be defined to help completing this information. As in the FIDL, constraints impose restrictions to the whole mission, while objectives guide the optimization of the mission.

A mission intent in MIDL follows the notation below:

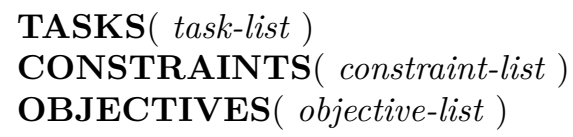

The task-list, constraint-list, and objective-list are lists of tasks, constraints, and objectives, respectively, separated by semicolons. These elements of the MIDL are covered in detail in the following sections.

\subsubsection{Tasks}

Tasks conform the backbone of any mission specification. A MIDL task comprises four elements:

1. A task identifier. This identifier consists of an alphanumeric sequence preceded by a hash sign (\#). Task identifiers allow referring to a specific task from other elements of the mission intent. They usually provide significant names that describe the task, such as '\#explore_area1' or '\#go_back_to_base'.

2. The candidate aircraft, that is, the aircraft that must complete the task. It may be specified using a particular aircraft identifier, or a condition on the type of aircraft, payload, or aircraft performance information (e.g., mass, maximum operating altitude, or maximum speed).

3. A precondition. The precondition describes the circumstances that enable the execution of a task. A precondition may refer to the state of the candidate aircraft or to the execution status of this or another task.

4. An effect describing the behaviour of the aircraft during the execution of the task.

The information about the candidate aircraft is used by the mission planner to determine which aircraft can perform each task. For example, the candidate aircraft may specify an aircraft identifier (e.g., 'ID0814B'), a required payload (e.g., 'equips(camera)'), a type of aircraft (fixed-wing or quadrotor), or a maximum weight (e.g., ' $m \leq 5 \mathrm{~kg}$ '). It is possible to combine several conditions using boolean operators. If a task requires a quadrotor aircraft that can fly at 
$10 \mathrm{~m} / \mathrm{s}$, this can be denoted as 'type (quadrotor) $\wedge v_{h}^{\max } \geq 10 \mathrm{~m} / \mathrm{s}$ '. If all aircraft are eligible to perform a task, the candidate aircraft can be defined as 'any'.

Preconditions provide the mission planner with information on whether a task can be started at a specific time during the mission. If the precondition of a task is met again after the task has been completed, the task may be executed again. It is also possible to specify the minimum and maximum number of times a task can be executed throughout the mission.

Using the notation in appendix $\mathrm{D}$, the following examples provide valid preconditions:

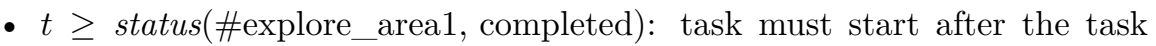
'\#explore_area1' has been completed.

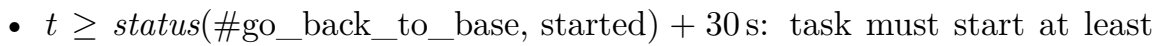
30 seconds after the task '\#go_back_to_base' has started.

- $t \leq 600$ s: task must start within the first 10 minutes of the mission.

- $z_{e} \geq 30 \mathrm{~m}$ : task must start when candidate aircraft is at least 30 metres above ground.

The status function returns the time (with respect to the start of the mission) at which the event occurred (or 0 if the event didn't occur yet), so it must be compared against the current time, $t$. Note that this function always refers to the last time the status is reached. If a completed mission starts again, then the starting time will be greater than the completion time.

The effect of a task is specified using a flight intent expressed in FIDL, potentially including its own constraints and objectives, which are local to the task. The effect may also be specified using a template and all the required parameters. As it will be seen, a fully-defined template (which includes all the necessary parameters), can be translated into a flight intent, so both definitions are equivalent.

The entire notation of a task (which is detailed in appendix D) can be summarized as:

\{ effect; candidates; precondition; min; max $\} \#$ id

where effect is the task effect (usually defined using a template), candidates is the specification of the candidate aircraft, precondition is the precondition of the task, which must be executed between min and max times. Finally, $i d$ is the task identifier.

\subsubsection{Templates}

Templates play a similar role in the MIDL to that of ICDL templates. A MIDL template describes the effect of a frequently used task that can be easily described in a few words, such as 'go to a location' or 'survey an area'. The template contains all the information on how to perform a task, save some parameters. These parameters depend on the particularities of the task (e.g., the location to be reached or the area to be surveyed). Templates can then be 
used to define the effect of a task by just specifying the name of the template and all the necessary parameters.

Depending on the complexity of the templates, they can be classified as simple or algorithmic templates.

\section{Simple templates}

When defining a mission, it is common to have several tasks whose effects are practically identical, except for some variables or magnitudes. These effects can be written once as a simple template, and then be used in all tasks by just referring to it. The elements in the template that vary between tasks are left unspecified and become the parameters of the template.

Simple templates follow the rules of the FIDL, but most elements in the flight intent (e.g., magnitudes, specifiers, waypoints, etc.) can be declared as template parameters. These parameters remain undefined until the template is instantiated when used to define a task.

One example of simple template is the 'Go-to' template, which commands the candidate aircraft to reach a point, with time restrictions. It is defined as:

\section{FLIGHT_SEGMENTS $(\langle\mathrm{WPT}\rangle)$}

CONSTRAINTS $\left(t^{\min } \leq t \leq t^{\max } @ \mathrm{WPT}\right)$

This template has three parameters:

1. WPT: the destination to be reached, a waypoint specifying a location.

2. $t^{\text {min }}$ : a time after which the candidate aircraft must reach the waypoint in order to fulfil the task.

3. $t^{\max }$ : a time before which the candidate aircraft must reach the waypoint in order to fulfil the task.

The two parameters, $t^{\min }$ and $t^{\max }$, define a time window during which the task must be completed. This is useful, for example, when the mission requires taking a photo of a target at a specific time.

In order to instantiate the template described above, it is necessary to provide the name of the template, with all the necessary parameters. In the notation of appendix D, it can be written as 'Go-to $\left\langle\mathrm{WPT}, t^{\mathrm{min}}, t^{\mathrm{max}}\right\rangle$ '.

\section{Algorithmic templates}

Some recurring tasks are too complex to be defined as a FIDL word with some parameters unspecified. For example, it would be convenient to have a template that commands an aircraft to survey some area. However, in this template, the actual path followed by the aircraft depends on the size and shape of the area. Some calculations are necessary to compute the number and position of the intermediate waypoints that must be traversed to cover the entire area.

Algorithmic templates allow to programmatically define the FIDL output of a template given a set of parameters. That is, algorithmic templates process 
the input of the template (its parameters) and produce a FIDL word as output, which constitutes the task's effect. This provides much more semantic power to the templating mechanism of the MIDL.

As discussed above, one example of algorithmic template is the 'survey' template. This template has three parameters:

1. $\left\{W_{1}, \ldots, W_{n}\right\}$ : the area to be surveyed, a polygon defined as a list of waypoints.

2. $h$ : an altitude to be maintained during the surveillance.

3. $t^{\text {end }}$ : the time that must be spent surveying the area.

This template can be instantiated by providing those parameters. Using the notation in appendix $\mathrm{D}$, it can be written as 'Survey $\left\langle\left\{W_{1}, \ldots, W_{n}\right\}, h, t^{\text {end }}\right\rangle$ '.

\subsubsection{Constraints}

In addition to the constraints associated to each particular task, it is possible in the MIDL to define constraints that affect the whole mission.

Constraints in a mission intent can be interpreted in two different ways: Most commonly, they represent FIDL constraints that apply to more than one task. For example, they may indicate no-fly zones, airspace constraints, airway segments, etc. These type of constraints are denoted global constraints. Global constraints should be handled by the trajectory predictor (or path planner), affecting the mission planner only indirectly. The notation and semantics of global constraints is identical to those of FIDL constraints.

On the other hand, constraints in a mission can also represent constraints that must be handled by the mission planner. These are denoted mission constraints. Mission constraints involve several aircraft or tasks. These constraints' effect may involve relationships more complex than allowed in the FIDL.

The notation of mission constraints differs slightly from FIDL constraints. In the notation of appendix $\mathrm{D}$, they are written as:

$$
\text { declaration }_{1}, \ldots \text {, declaration }, \text { effect @ DoA }
$$

Mission constraints start with zero or more declarations specifying the involved aircraft, separated by commas. Declarations start by defining an alias for that group of aircraft, and then describe the aircraft included in the group using the same notation as the one used to specify candidate aircraft in tasks. Therefore, it is possible to refer to a specific aircraft by its aircraft identifier, or a group of aircraft based on their payload, type of aircraft, or other properties. For example, $U \in$ type(fixed-wing) declares that the constraint affects fixed-wing aircraft, and assigns the name $U$ to denote them. This notation is used within the effect and DoA of the constraint to refer to the state vector of a particular aircraft. For example, $v_{g}(U)$ refers to the ground speed of aircraft $U$.

The DoA in a mission constraint defines the conditions that must be met for the constraint to be active. This condition may depend on the state vector 
of the involved aircraft, the status of a task (using the status function), or the properties of the aircraft.

The effect of the mission constraint imposes a constraint that must be met when the constraint is active (i.e., when the conditions in the DoA are met). When some groups of aircraft are declared, special operands are required to refer to all the aircraft in the group. These special operands include existential quantification ('there exists', $\exists$ ), universal quantification ('for all', $\forall$ ), summation operations $\left(\sum\right)$, etc. It is possible to reference a task's status in a constraint, using the status function defined previously. Additionally, it is possible to reference all tasks simultaneously (for example, in an universal quantification), using the hash sign (\#).

These are some examples of mission constraints:

- $U=$ any, $V=$ any, $\forall_{U, V} \operatorname{dist}(U, V) \geq 5 \mathrm{~m} @ U \neq V$

The constraint above declares two aliases, $U$ and $V$, that represent any two aircraft in the mission. The effect of the constraint states that the distance between any those two aircraft must be at least 5 metres. The dist function computes the euclidean distance between two aircraft:

$$
\operatorname{dist}(U, V)=\sqrt{\left(x_{e}(U)-x_{e}(V)\right)^{2}+\left(y_{e}(U)-y_{e}(V)\right)^{2}+\left(z_{e}(U)-z_{e}(V)\right)^{2}}
$$

The DoA limits the application of the effect to the case where $U \neq V$, as otherwise this constraint would be unsatisfiable.

- $\forall \# \operatorname{status}(\#$, completed $) \leq 600 \mathrm{~s} @ \Omega$

This constraint states that all tasks must be completed within the first 10 minutes (600 seconds) of the mission. The universal DoA, represented by $\Omega$, makes the constraint active at all times.

\subsubsection{Objectives}

Objectives represent optimization goals in the form of mathematical expressions that must be minimized. The MIDL allows defining objectives that affect the whole mission. Unlike MIDL constraints, all MIDL objectives must be handled by the mission planner. Objectives may also contain declarations specifying the aircraft subject to a particular optimization, using the same notation of mission constraints.

The notation of MIDL objectives is analogous to that of FIDL objectives, with the addition of declarations, and some additional operators (e.g., summation, product). In general, MIDL objectives are expressed as:

$$
\text { (weight) declaration } 1, \ldots, \text { declaration }, \text { effect } @ D o A
$$

Mission objectives may involve a group of aircraft, which is defined using a declaration. These declarations are analogous to the declarations used in mission constraints: an alias is defined for the group of aircraft, and the aircraft integrating that group are specified using their state, type, or payload. The effect is represented by a function describing the cost of the mission per time unit. The aircraft in each of the declared groups can be included in the effect 
using the summation $\left(\sum\right)$, product $\left(\prod\right)$, maximum, or minimum operators by using the alias assigned to the group. It is also possible to use those operators to reference all the tasks in the mission by using the pound sign (\#) instead. The DoA is a logical condition defining the time during which the objective is active. The effect must be integrated during the time defined by the DoA, obtaining the cost to be minimized.

As stated before, the effect in a mission objective may affect a group of aircraft defined using a declaration.

The following are examples of MIDL objectives:

- (1) $U=$ type(fixed-wing), $\sum_{U}-\mathrm{d} m_{f}(U) / \mathrm{d} t @ \Omega$

This objective minimizes the total fuel consumption of all fixed-wing aircraft during the whole mission. The number in parenthesis at the beginning of the objective indicates the weight of this objective.

- (1) $\sum_{\#}(\operatorname{status}(\#$, completed $)-\operatorname{status}(\#$, started $)) @ \operatorname{status}(\#$, started $) \leq$ status (\#, completed)

This objective minimizes the accumulated duration of all tasks, taking into account only those tasks that are completed.

Note that the final cost function for optimizing the mission does not only take into account the mission objectives, but also must consider all the objectives included in each task. Therefore it is of paramount importance that objectives are appropriately weighted to obtain the desired results.

\subsection{Examples}

In this section, some complete examples of mission intent expressed in the MIDL are provided. These examples use the notation outlined along this chapter, which is described in more detail in appendix D.

A simple example of mission intent requires two targets to be reached during the first 20 minutes since the start of the mission. This can be expressed in MIDL as:

TASKS (

$\{$ Go-to $\langle\mathrm{WP} 1,0 \mathrm{~s}, 1200 \mathrm{~s}\rangle ;$ any $; t \geq 0 ; 1 ; 1\} \#$ go_to_WP1;

$\{$ Go-to $\langle\mathrm{WP} 2,0 \mathrm{~s}, 1200 \mathrm{~s}\rangle ;$ any; $t \geq 0 ; 1 ; 1\} \#$ go_to_WP2 )

OBJECTIVES $\left((1) U=\right.$ any, $\left.\sum_{U} 1: \Omega\right)$

In this example, both points must be reached in the interval between 0 and 1200 seconds (that is, 20 minutes). There are no restrictions to which aircraft can perform each task, and the preconditions of both tasks are trivial, as they're always met. Each task must be completed exactly one time. In this case, it isn't necessary to include any constraint forcing all tasks to be competed within 20 minutes, as each task includes that condition. The mission includes an optimization objective, though. This objective minimizes the total flight time by all aircraft in the mission. 
A feature of the MIDL that will be later used in this thesis it the possibility of defining tasks that must be repeated several times during the mission. For example, a mission may require a photography of one particular place to be taken every 60 seconds (or less). The MIDL allows specifying these kind of tasks, as shown in the example below.

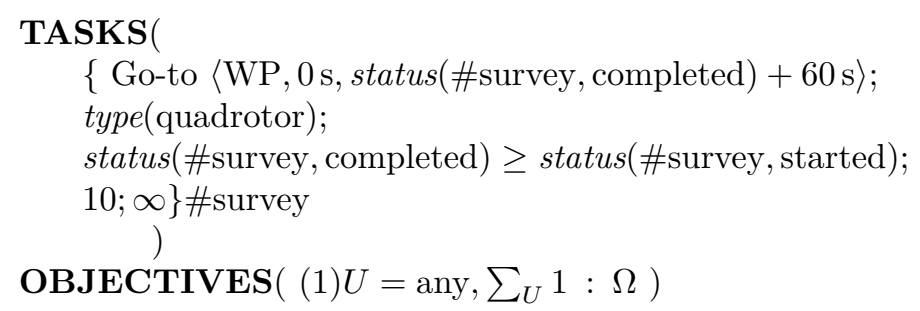

In this example, the effect of the task states that it must be completed not later than 60 seconds after it was completed for the last time. Remember that the status function will return zero if the event has never occurred, so the task must be completed for the first time within the first 60 seconds of the mission.

The candidate aircraft, in this example, is limited to quadrotor aircraft using the type function.

The precondition of this task prevents the task from being started before the previous execution is completed. Otherwise, a mission planner could execute the task with two aircraft simultaneously. The minimum and maximum number of executions force the task to be executed at least 10 times.

\subsection{Final words}

This chapter has presented the MIDL, a language for describing missions involving several aircraft. Despite the common name, this MIDL is unrelated to the language introduced in [74]. Both languages are designed to be used in combination with the FIDL. However, the language described in this chapter is an extension of the FIDL - meaning that anything that can be expressed in the FIDL can be expressed in the MIDL_-, while the language in [74] isn't.

A mission intent expressed in MIDL could completely and unambiguously describe the trajectories of every aircraft involved. In this extreme case, each task would describe a whole aircraft's trajectory using FIDL (which, in turn, would use the ICDL and AIDL to achieve the desired level of detail).

The reader should also note that the description of the MIDL provided in this chapter corresponds to a language that is still under development. New semantics may be added in the future, which could simplify the notation - making the language easier to read by a human operator - or increase its expressive power -by making it possible to express things that were previously impossible.

The MIDL is the highest level in the hierarchy of intent-related languages described in this thesis. This hierarchy has shown how it is possible to use formal languages to describe aircraft trajectories with an arbitrary level of detail, which make the languages in the hierarchy an ideal exchange format to communicate disparate systems: from a mission planner to a trajectory predictor, a surveillance system, etc. 


\section{Chapter 10}

\section{Surface movement description language}

The surface movement description language (SMDL) [129] is a formal language that can be used to describe the movement of aircraft (and potentially other vehicles) on airport surface. This language can be used by routing and guidance functions to exchange information related to airport surface operations.

The SMDL is based on the ground clearances for surface operations defined by the ICAO [13]. A clearance is a guidance instruction given by the ATCO that the flight crew (or driver) must follow. These instructions include taxiing to a waypoint, vacating a runway, or taking off. The SMDL can be used to represent a list of instructions to be performed sequentially by an aircraft or vehicle. This list of instructions is called a route. When completely defined, a route expressed in SMDL provides a sequence of instructions defining the route from the origin to the destination, containing all the intermediate points and the time each instruction is completed. However, many of the systems involved in routing and guidance functions need to represent potential sources of uncertainty in surface movements. Some types of uncertainty information are supported by the SMDL, so that routes can be defined with varying levels of detail. A simple route expressed in SMDL would only contain the initial position and the target destination.

Although the SMDL is not included in the hierarchy of languages described in previous chapters, it has similar features. For example, they are designed to allow describing trajectories with varying levels of detail, and in a way that is readable by both humans and computers. The most notable differences of the SMDL with respect to those languages are direct consequences of the scenario to which it focuses on. In airport surface, movement has fewer DoFs, aircraft can be completely still, and they usually move slower than while airborne. Also, a trajectory description based on ground clearances (i.e., SMDL) isn't as accurate as one based on mechanical constraints (i.e., AIDL, FIDL, etc.): additional information is required in order to predict a trajectory based on a route expressed in SMDL. However, the level of detail provided by the SMDL is deemed sufficient for the purposes it was designed for [129].

In the remainder of this chapter, all the features of the SMDL are described 
and examples are provided.

\subsection{Language description}

As previously stated, a word in SMDL is called a route. Routes are sequences of SMDL instructions that define surface operations to be performed successively. All the instruction types available and the rules that allow combining these instructions to form routes are described in this section.

The SMDL can be used to represent uncertainty in surface operations. In particular, the following two types of uncertainty are supported:

- Time uncertainty: most instructions include the time at which the associated operation is completed. This can be used to indicate when an aircraft will reach a certain point in the airport surface, when it will take-off, etc. Times are expressed as intervals $\left(t_{1}, t_{2}\right)$, where $t_{1} \leq t_{2}$, and meaning that the operation must finish between $t_{1}$ and $t_{2}$. Therefore, when $t_{1} \neq t_{2}$, the instruction doesn't provide a specific time.

- Operation uncertainty: in general, every instruction in a route corresponds unequivocally to one surface operation. However, a special instruction - the empty instruction - does not correspond to any operation. This instruction can be used to represent fragments of the route where the operations are unknown.

The SMDL imposes limits to these ways of representing uncertainty to ensure the route is ambiguous only when intended.

As for time uncertainty, times cannot decrease along the route, that is, if one interval $\left(t_{3}, t_{4}\right)$ appears in some instruction after another interval $\left(t_{1}, t_{2}\right)$, then $t_{1} \leq t_{3}$ and $t_{2} \leq t_{4}$. This is the same as saying that an instruction can't start before a previous instruction, and it can't finish after a later instruction. Operations are performed in the same order as they appear in the route.

A route can be divided in two segments: a cleared segment and a non-cleared segment. Instructions in the cleared segment have been issued to the aircraft by the ATCO and they are considered definitive. Therefore, cleared segments can't contain any empty instructions. On the other hand, the non-cleared segment is subject to future change and it may contain empty instructions.

\subsubsection{Instructions}

Instructions define simple aircraft operations on airport surface, such as driving along a taxiway segment or stop and wait on a certain location. Any two consecutive instructions are tightly bonded, in the way that after completing one instruction there is only one way of performing the next one. For example, if two consecutive instructions reference two waypoints of the route, both waypoints must be adjacent (so that the path between both points is unambiguous).

Many of these instructions require some parameters to fully define the expected behaviour. Most instructions - and some of their parameters - are based on a formalization of the airport surface clearances described by the ICAO [13]. 
Also, many instructions require a time interval parameter. All the times in a SMDL instance are referenced to the same reference time. Sometimes, a time interval may not be available from routing information sources. In such cases, the time interval parameter is left unspecified, which means it is left as wide as it can possibly be without affecting any other time interval.

There are two special instructions that do not correspond to any clearance or surface operation: the empty and the cleared instructions. These two instructions compose the special instruction category. There are a total of four instruction categories, which are detailed next.

\section{Special instructions}

These instructions denote elements of the language that do not have a fully defined meaning by themselves.

1. Empty. The empty instruction represents a segment of the route where some information remains unspecified. Therefore, there may be several ways of achieving the next instruction. For example, this instruction may be used when the path between two nodes is not defined or there is not enough information to complete the route between the origin and the destination.

2. Cleared. This instruction does not define any operation; it just indicates the end of the cleared segment. All instructions before the cleared instruction compose the cleared segment. If a SMDL word starts with a cleared instruction, then the cleared segment is assumed to be empty. It should be noted that, in this case, no surface movement should be performed before the reception of a clearance.

\section{Initial instructions}

These instructions represent the ways aircraft may start surface operations. The first non-special instruction in a route must be an initial instruction.

3. Landing. The landing instruction indicates the aircraft is in the glide path arriving to the runway. This instruction's parameters are the runway identifier (RWY_ID), to be able to support airports with several runways, and the time interval $\left(t_{1}, t_{2}\right)$ when the aircraft is expected to land. A vacate instruction (to be described later) must follow to indicate the point at which the plane will exit the runway.

4. Init. The init instruction will be used if the aircraft is already on airport surface with the engines started (for instance, after going through a de-icing or maintenance process). This instruction indicates the initial position of the route for non-landing aircraft. The instruction shall contain a parameter with the identifier for the initial location (a waypoint identifier WPT_ID) and the time interval $\left(t_{1}, t_{2}\right)$.

5. Start-up. Start-up instruction will be used when the aircraft is initially in a stand, or at any other position, provided aircraft engines are turned-off. 
This instruction indicates the aircraft to start-up the engines. The time interval $\left(t_{1}, t_{2}\right)$ is provided. An init instruction must follow to indicate of the initial position of the route.

Initial instructions are summarized in table 10.1.

\begin{tabular}{l|c|c} 
Instruction & Parameters & Time interval \\
\hline \hline Landing & RWY_ID & $\checkmark$ \\
Init & WPT_ID & $\checkmark$ \\
Start-up & & $\checkmark$
\end{tabular}

Table 10.1: Summary of initial instructions

\section{In-route instructions}

The in-route instruction category comprises all the instructions that may take place anywhere within the route. The taxi, wait, cross, vacate, de-ice, enter, and line-up instructions belong to this category.

6. Taxi. The taxi instruction indicates the aircraft to drive from the current position to the location defined by WPT_ID. It also contains the time interval $\left(t_{1}, t_{2}\right)$ to end the maneuver. No intersections or diversions may exist between the current position and WPT_ID, so that the instruction is unequivocal.

7. Wait. The wait instruction indicates the aircraft the obligation of stopping at a certain point for a defined time interval. WPT_ID indicates the location where the wait instruction must be completed. The second parameter is the time interval $\left(t_{1}, t_{2}\right)$ at which the wait must end.

8. Cross. The cross instruction is a variation of the taxi instruction, that indicates the aircraft has to drive from the actual position to a certain point, but crossing a runway in the process. RWY_ID is the identifier of the runway to be crossed, WPT_ID is the point where the manoeuvre will end, and $\left(t_{1}, t_{2}\right)$ is the time interval to end it.

9. Vacate. The vacate instruction indicates the aircraft to exit the runway protection area, usually after a landing. RWY_ID specifies the runway where the landing has taken place. WPT_ID contains the identifier of the point where the aircraft must exit the runway. The time interval $\left(t_{1}, t_{2}\right)$ to complete the instruction is also provided.

10. De-ice. This is an instruction for aircraft in the winter period and when a de-icing platform is available at the airport. The instruction shall contain a parameter with the identifier for the location of the de-icing platform (WPT_ID) and the time interval $\left(t_{1}, t_{2}\right)$.

11. Enter. The enter instruction allows a mobile to access the runway protection area. RWY_ID is the identifier of the runway to which access has been permitted. Also, the time interval $\left(t_{1}, t_{2}\right)$ is provided. 
12. Line-up. The line-up instruction indicates the aircraft has to drive from its current position to a certain point inside the runway protection area, where it should wait before it is cleared for take-off. RWY_ID specifies the runway. WPT_ID is the identifier of the point where the aircraft must line up. The time interval $\left(t_{1}, t_{2}\right)$ to complete the instruction is also provided.

In-route instructions are summarized in table 10.2.

\begin{tabular}{l|c|c} 
Instruction & Parameters & Time interval \\
\hline \hline Taxi & WPT_ID & $\checkmark$ \\
Wait & WPT_ID & $\checkmark$ \\
Cross & RWY_ID \& WPT_ID & $\checkmark$ \\
Vacate & RWY_ID \& WPT_ID & $\checkmark$ \\
De-ice & WPT_ID & $\checkmark$ \\
Enter & RWY_ID & $\checkmark$ \\
Line-up & RWY_ID \& WPT_ID & $\checkmark$
\end{tabular}

Table 10.2: Summary of in-route instructions

\section{Final instructions}

Final instructions represent the ways aircraft may end surface operations. The last non-special instruction in a route must be a final instruction. The final instructions are the take-off and the end instructions. If the route ends when the aircraft takes off, then the take-off instruction is used. Otherwise, the end instruction is used.

13. Take-off. The take-off instruction should always be preceded by a lineup instruction. It is the final surface instruction for a departing aircraft. RWY_ID is the identifier of the runway from which the aircraft departs, and $\left(t_{1}, t_{2}\right)$ is the time interval for the aircraft to become airborne.

14. End. This instruction indicates the end of a movement on airport surface. This instruction can be seen as a final delimiter and therefore no parameters are needed.

Final instructions are summarized in table 10.3.

\begin{tabular}{l|c|c} 
Instruction & Parameters & Time interval \\
\hline \hline Take-off & RWY_ID & $\checkmark$ \\
End & &
\end{tabular}

Table 10.3: Summary of final instructions

\subsubsection{Routes}

Basically, routes are sequences of instructions. Nonetheless, not any sequence of instructions is a valid route. The rules governing the formation of routes are presented in this section. 
1. The cleared instruction must appear exactly once in every route.

This instruction may appear at the beginning of the sequence, meaning the cleared segment has no instructions; it may appear at the end, which means the whole sequence is a cleared segment; or it may appear somewhere in the middle, meaning some of the instructions belong to the cleared segment and some to the non-cleared segment.

2. The first instruction in the route - other than the cleared instructionmust be an initial instruction. Instructions in the initial category represent the ways surface operations may start.

3. The last instruction in the route - other than the cleared instructionmust be an final instruction. Instruction in the final category represent the ways surface operations may finish.

4. Non-special instructions in a route (i.e., those representing a clearance) are sorted chronologically, in the same order that the associated surface operations must be performed.

5. No empty instruction may appear before the cleared instruction. This rule ensures that the cleared segment doesn't have any empty instructions.

6. Any empty instruction must be followed by a taxi instruction. Therefore, it is possible to know, after an unknown operation, where the aircraft resumes its route.

7. Any landing instruction must be followed by a vacate instruction. This is necessary to indicate the waypoint where the aircraft leaves the runway.

8. Any start-up instruction must be followed by a init instruction. This is necessary to indicate the initial position of the aircraft.

9. Any take-off instruction must be preceded by a line-up instruction. Due to airport procedures, the aircraft is cleared to enter the runway before a take-off authorization is received.

\subsection{Graphical representation}

A graphical representation can be used to express routes in SMDL. Instructions are represented as rounded boxes with a color that depends on the instruction type, as shown in figure 10.1. The instruction name and all its parameters are contained in their respective box. Routes are represented as stacks of instructions, where the instruction at the top is the first of the sequence. Examples of SMDL routes using this notation are provided in section 10.3 .

\subsection{Examples}

The description and notation of the SMDL will be illustrated with several examples included in this section. 


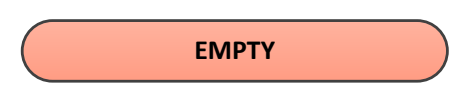

(a) Empty instruction.

\section{LANDING RWY_ID, $\left(t_{1}, t_{2}\right)$}

(c) Landing instruction.

$$
\text { START-UP }\left(t_{1}, t_{2}\right)
$$

(e) Start-up instruction.

WAIT WPT_ID, $\left(t_{1}, t_{2}\right)$

(g) Wait instruction.

$$
\text { VACATE RWY_ID, WPT_ID, }\left(t_{1}, t_{2}\right)
$$

(i) Vacate instruction.

ENTER RWY_ID, $\left(t_{1}, t_{2}\right)$

(k) Enter instruction.

TAKE-OFF RWY_ID, $\left(t_{1}, t_{2}\right)$

(m) Take-off instruction.

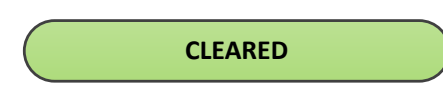

(b) Cleared instruction.

INIT WPT_ID, $\left(t_{1}, t_{2}\right)$

(d) Init instruction.

TAXI WPT_ID, $\left(t_{1}, t_{2}\right)$

(f) Taxi instruction.

CROSS RWY_ID, WPT_ID, $\left(t_{1}, t_{2}\right)$

(h) Cross instruction.

DE-ICE WPT_ID, $\left(t_{1}, t_{2}\right)$

(j) De-ice instruction.

(l) Line-up instruction.

\section{END}

(n) End instruction.

Figure 10.1: SMDL instructions. 


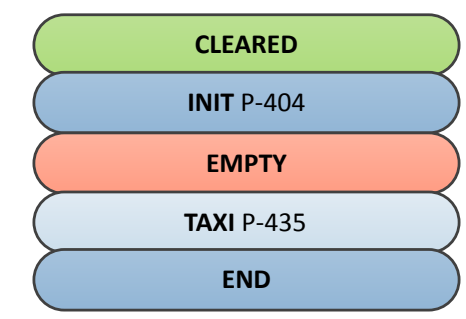

Figure 10.2: Simple SMDL route.

These examples start with one of the simplest routes that can be expressed in SMDL, shown in figure 10.2. This route represents the intent of going from one waypoint to another. Despite its simplicity, this route meets all the rules of SMDL. It contains one instruction from the initial and final categories (the init and end instructions, respectively). The two points are not adjacent (i.e., there are intersections between them, and therefore multiple paths between the points), so an empty instruction must be placed between them to indicate the ambiguity in the route. The cleared instruction appears before the empty instruction, as it is the first instruction in the route. In this case, the aircraft (or vehicle) shouldn't start any operation, as no clearance has been received and the intermediate path is still unknown. None of the instructions specify any time intervals, meaning these intervals are as wide as possible -in this case, $(0, \infty)$

A more complex example is presented next. The following example shows a completely defined route on the Adolfo Suárez Madrid-Barajas (LEMD) airport. Figure 10.3 shows a portion of the map of this airport with the route. This route can be expressed in SMDL as the sequence of instructions shown in figure 10.5a. The cleared segment includes all the instructions from the start-up at stand 534 to a wait at waypoint P-437. This segment corresponds to the green part of the route in figure 10.3. Instructions in the cleared segment are confirmed, and can therefore be issued to the flight crew. The non-cleared segment includes the remaining instructions until take-off at runway $36 \mathrm{R}$. This segment corresponds to the blue part of the route. Instructions in this segment may still change, as they have no confirmation from the ATCO. This route is completely defined, that is, there is no operation uncertainty. Most instructions include a time interval with the estimated completion times of each operation.

A similar example can be defined in which some parts of the route are still unknown. This example route is shown in figure 10.4, and the corresponding SMDL can be found in figure 10.5b. The route starts at the same stand. In this case, the cleared segment (in green) includes only the instructions until the waypoint $\mathrm{P}-434$ is reached. The non-cleared segment contains a taxi instruction to continue to waypoint P-435. The route continues to waypoint $\mathrm{P}-80$ following a route that is undefined; only one intermediate waypoint (P-438) is defined. The undefined fragments are depicted as red dotted straight lines. These lines connect the start and end of the undefined route, but do not represent the actual route followed by the aircraft. After the undefined sections, waypoint P-80 is reached and the route is completely defined from that point until take-off. 


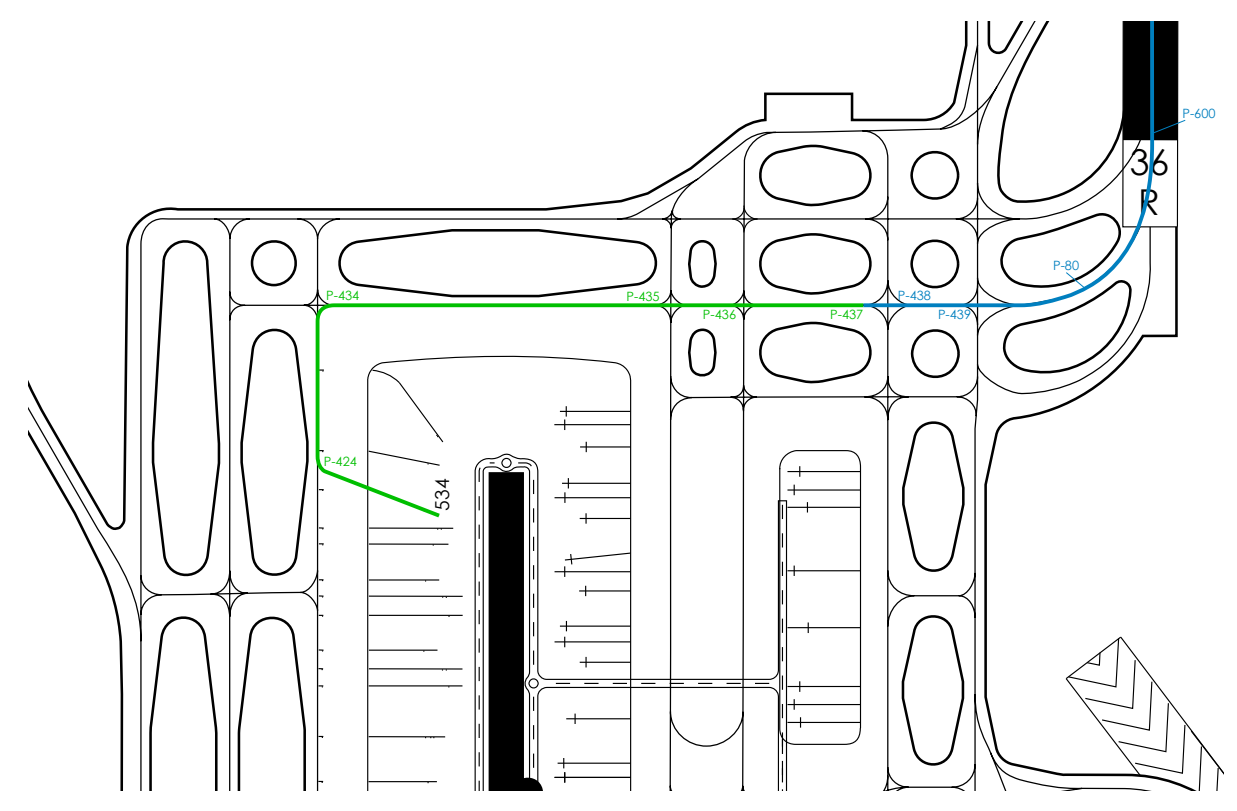

Figure 10.3: Completely defined aircraft route on the airport surface map.

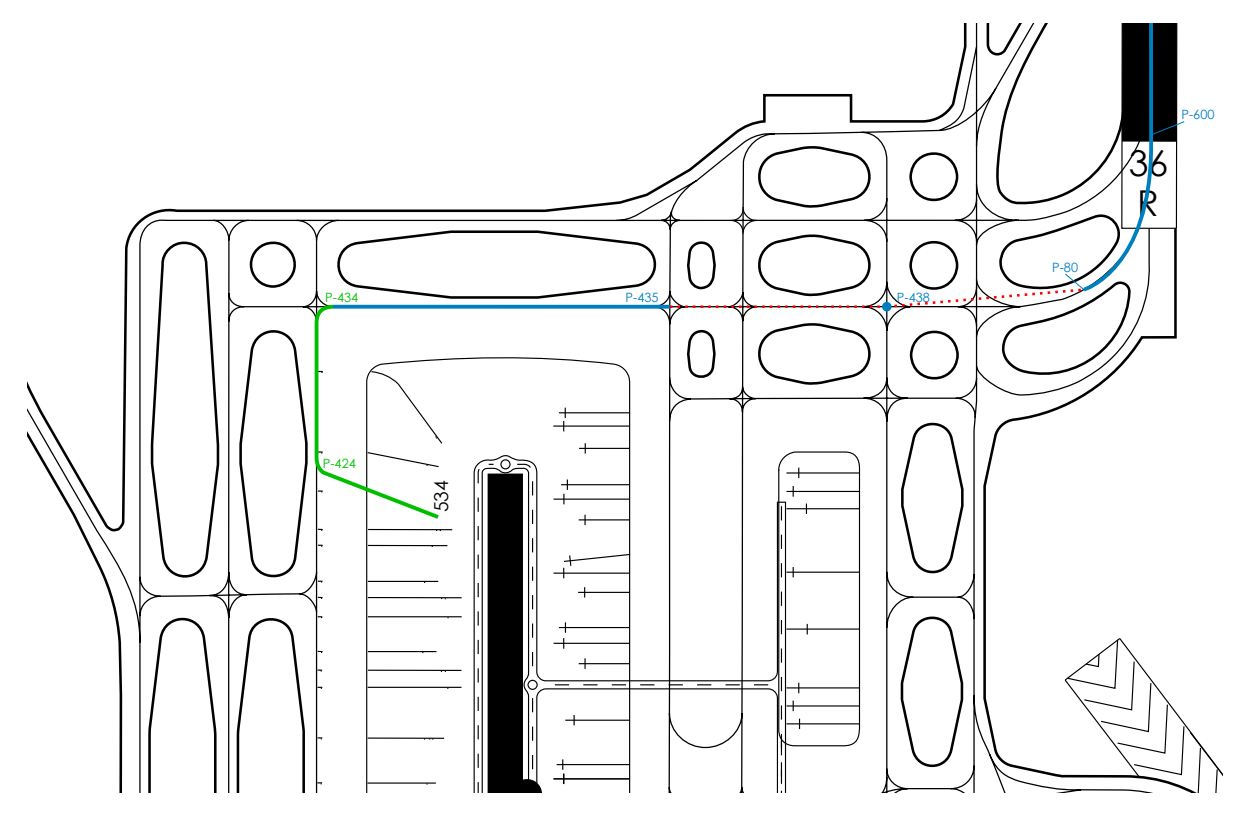

Figure 10.4: Partially defined aircraft route on the airport surface map. 


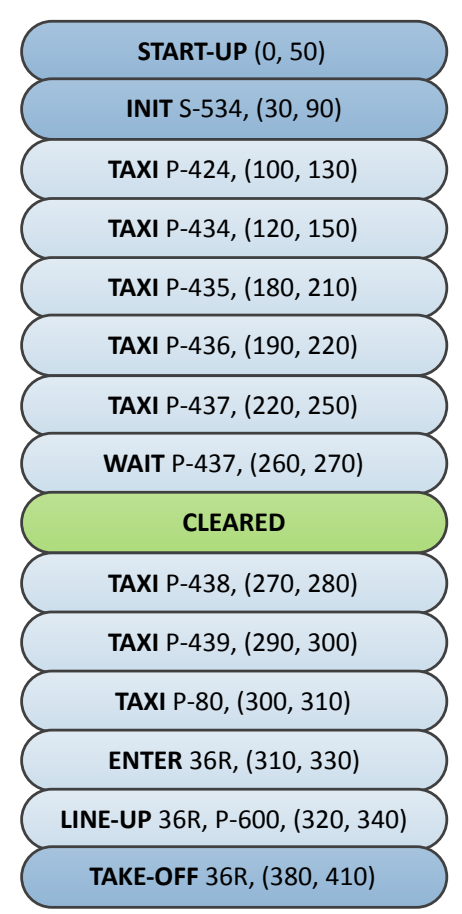

(a) Completely defined route.

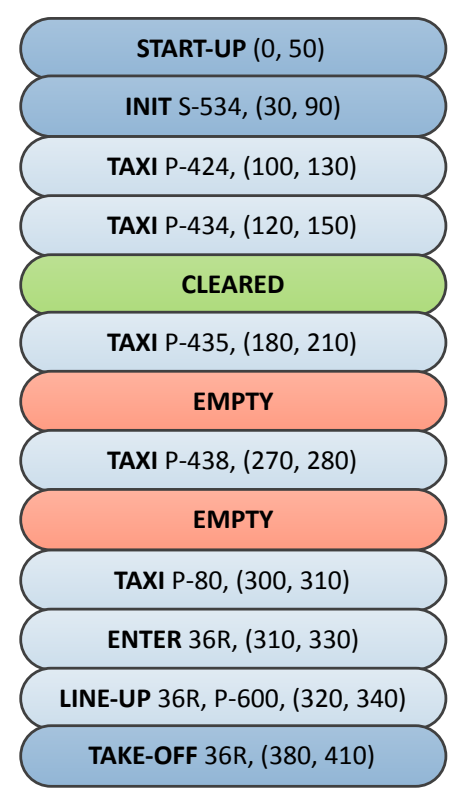

(b) Partially defined route.

Figure 10.5: Aircraft routes in Adolfo Suárez Madrid-Barajas airport.

\subsection{Final words}

The formal language introduced in this chapter completes the collection of languages aimed at describing aircraft trajectories. In this case, the language is built from the airport surface clearances, an approach substantially different from all the other languages described in this thesis, which were based on mechanical constraints to the aircraft motion. Although this approach is less precise - i.e., it is not possible to predict the exact position of an aircraft at any time given its SMDL route-, the level of detail it can provide is sufficient for most functions of the A-SMGCS. In particular, to routing, guidance, and safety nets.

Some important advantages of using this language as a standard way for sharing route information, are the following:

- Collaboration. The SMDL allows to place 'gaps' in the route (as empty instructions), that will later be specified. Therefore, several actors may share the responsibility of building a route by filling some of these 'gaps' and passing the route to the next actor. Ultimately, only the instructions in the cleared segment will be executed, so compatibility and safety checks can be performed before any clearance is issued.

- Modularity. A common, well defined interface for each function enables the development of drop-in replacements with fewer risks. For example, several routing functions can be easily switched, as long as they all provide 
the same output (in SMDL format) without affecting rest of the system.

- Consistency. The rules of the SMDL prevent some undesirable situations from happening, such as many types of route inconsistency. Moreover, the SMDL differentiates instructions that are safe to be performed (those in the cleared segment) from those still not confirmed to be safe. At the same time, it makes it possible to preview what operations are going to be performed next - although the preview may change or it may not be complete.

This language has been included for completion, and applications of this language are out of the scope of this thesis. However, its applications to routing and safety nets have been studied in [129], and applications to a broad range of functions within A-SMGCS can be found. 



\section{Part III}

\section{Applications to Aircraft \\ Management}





\section{Chapter 11}

\section{Trajectory prediction using formal languages}

Part II of this thesis was devoted to the description of a variety of formal languages that allow expressing intent of one or several aircraft. Part III focuses on some of the applications that such languages could have in several aspects of mission and flight management. In particular, it analyses how automatic processing of mission, flight, and aircraft intent - expressed in the languages described in this thesis - can be used to manage missions and individual flights.

This chapter introduces the concept of trajectory language processing engine (TLPE), an abstract representation of a function in which the main input and output are expressed in any of the languages in the language hierarchy defined in part II. Many of the functions in ATM and UAS can be formulated using this concept, as their main input and output consists of descriptions of a flight or a mission, which can be expressed in those languages.

The rest of the chapter focuses mainly on the applications of formal languages and, in particular, TLPEs to trajectory prediction in ATM. It will be shown how a trajectory predictor can be formulated as a TLPE. Moreover, the proposed trajectory predictor structure also formulates many of its sub-functions as simpler TLPEs, providing a modular design. The development of this trajectory predictor is the result of a collaboration with BR\&TE.

\subsection{Trajectory language processing engines}

Processing of intent is carried out by diverse functional modules, denoted trajectory language processing engines (TLPEs) or simply engines. As shown in figure 11.1, a TLPE manipulates an intent $I_{1}$, producing a different intent $I_{2}$. The languages used to express input and output intents $-L_{1}$ and $L_{2}$, respectivelymay or may not be the same.

Many processes related to aircraft management can be seen as a TLPE or a combination of several TLPEs. For example, a process that distributes the workload of a mission among the available aircraft is a TLPE taking a mission intent and producing as many flight intents as available aircraft. This mission 

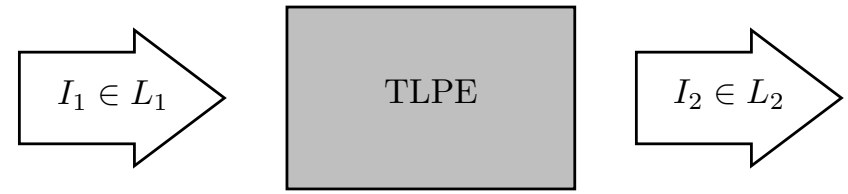

Figure 11.1: Trajectory language processing engine.

management process will be described in chapter 14. Another TLPE can be used to predict aircraft trajectories, translating a flight intent into an aircraft intent. This engine can be built as an aggregation of other TLPEs, as it will be shown in section 11.2. A different TLPE could be used to amend flight plans (e.g., to avoid a conflict or because of bad weather), therefore editing the input flight intent to generate an amended flight intent.

The concept of TLPE encompasses a wide range of intent processes with many different purposes. Depending on the purposes of TLPEs, they can be classified as enrichment, translation, transcription or edition engines [127].

\subsubsection{Enrichment engines}

Enrichment engines represent processes that have the same language as input and output (in the notation of figure 11.1, $L_{1}=L_{2}$ ) and complete the input intent by adding new information from other sources. Therefore, the output intent $I_{2}$ will contain all the information from $I_{1}$ plus some additional information. This information can be included by reducing the number of open parameters, including additional constraints or objectives, etc., depending on the language being processed. Enrichment engines are refinement processes that aim to reduce the DoF of some intent.

Some examples of enrichment engines are the following:

- The user preferences engine (UPE) enriches a flight intent with the relevant information from the user preferences. This engine is described in section 11.2.2.

- The operational context engine (OCE), described in section 11.2.3, adds the relevant data from the operational context to a given flight intent.

- The aircraft performance limitations engine (APLE) uses the data in the aircraft performance model (APM) to enrich a flight intent by adding performance-related constraints. More details are provided in section 11.2.4.

- The composite optimization engine (COE) closes the open interval and set parameters of an input composite by choosing the optimal (or nearoptimal) values. This engine is completely described in chapter 12 .

- The path planning engine (PPE) computes a 3D collision-free path in an environment with obstacles and no-fly zones. It is described in chapter 14 . 


\subsubsection{Translation engines}

The transformation of an intent $I_{1}$ expressed in a language $L_{1}$ to an equivalent intent $I_{2}$ expressed in a different language $L_{2}$ (thus, $L_{1} \neq L_{2}$ ) is performed by a translation engine. The output of translation engines may sometimes consist of a set of intents $\left\{I_{2}, I_{2}^{\prime}, I_{2}^{\prime \prime}, \ldots\right\}$ containing one intent per aircraft, in case the output describes the behaviour of several aircraft; or containing several possible intent for the same aircraft (i.e., alternate possible behaviours).

In many cases, the translation must complete the input intent to remove uncertainties. For example, when translating from ICDL to AIDL, interval and set parameters must be transformed into magnitude and specifier parameters, respectively, which contain more specific information. The output may be more specific than the input, but it can never be less specific. Therefore translation engines may require additional contextual information to remove uncertainties.

Some examples of translation engines are:

- The intent generation engine (IGE) is capable of translating a flight intent into an aircraft intent, as described in section 11.2.6.

- The trajectory computation engine (TCE) can translate an aircraft intent to a sampled trajectory. It is described in section 11.2.1 and chapter 13 .

- The trajectory prediction engine (TPE), described in section 11.2.7, is a TLPE that can translate either flight intent or aircraft intent into a sampled trajectory.

- The composite translation engine (CTE) translates an unambiguous composite into an aircraft intent. This translation is trivial in most cases, due to the nature of the ICDL and the AIDL. More details are provided in chapter 12 .

- The mission planning engine (MPE) takes a mission intent and computes a task distribution among the available aircraft, generating a flight intent for each of them. Chapter 14 provides details on this engine.

\subsubsection{Transcription engines}

A transcription is a transformation of some intent $I_{1}$ into an equivalent intent $I_{2}$ in the same language $\left(L_{1}=L_{2}\right)$. The two intents are equivalent, but they are usually not the same: they describe an equivalent behaviour using different expressions. This is useful in cases where some engine does not fully support a language, but only a subset of it - a dialect of the language. In these cases, an alternate intent must be expressed using only this dialect. The equivalence of the two intents can be conditioned to some restrictions; if these restrictions are not met, then $I_{1}$ and $I_{2}$ aren't equivalent.

Some examples of transcription engines are provided here:

- A flight control system (FCS) could use AIDL to guide an aircraft: instruction effects would become the guidance references, while triggers would be used to switch between these references. One problem of this approach is 
the use of auto triggers, which require iterating to be solved and cannot be handled by the FCS.

To solve this problem, a transcription engine can manipulate an AIDL word to remove auto triggers. This manipulation is achieved by computing the trajectory using a TCE to obtain the times each auto trigger is reached, and replacing auto triggers with such times. The equivalence of the two intents is conditioned to the use of the same APM and weather model (WM) used in this computation. The resulting intent can be provided to the FCS or any other engine that doesn't support auto triggers.

- The sampled trajectory provided by the TPE may provide only values for the minimum set of variables needed for the integration of the equations of motion. Also, the period (or frequency) between samples is determined by the TPE. A transcription engine can be used to interpolate samples in order to achieve the desired sampling period, and compute some state variables that weren't originally provided by the TCE. This manipulation of the sampled trajectory can be performed more accurately if the transcription engine has access to the APM, WM, aircraft intent, or other data.

\subsubsection{Edition engines}

Edition engines modify or replace some elements of the input intent $I_{1}$ to produce the output intent $I_{2}$. Both intents are expressed in the same language $\left(L_{1}=L_{2}\right)$. In this case, the input and output intents have the same level of detail (e.g., the same open DoF), but different information. The main difference between edition and enrichment engines is that the latter cannot change existing information, only add new information.

For example, an edition engine can change some elements in a flight intent to avoid a conflict. In this case, waypoints may be added or replaced, and new constraints may be placed in the resulting flight intent. In order to validate the output flight intent, a TPE should be used to ensure the resulting trajectory is conflict-free.

Other examples can be as simple as an edition engine that replaces an end trigger representing a required time of arrival (RTA) - that is, a time at which the aircraft must reach some waypoint - due to a delay of some sort (delayed take-off, traffic, etc.); or the inclusion of a holding pattern.

\subsection{Engine descriptions}

One of the most immediate applications of TLPEs is the prediction of aircraft trajectories. A trajectory predictor, after all, is a function that produces a detailed description of an aircraft trajectory from a higher-level description, such as a flight plan. This input and output can be expressed using the languages described in part II.

Furthermore, there are many sub-functions within a trajectory predictor that can also be expressed as a TLPE. In fact, the complete architecture of a 
trajectory predictor can be described as a composition of several TLPEs, each performing one of the aforementioned sub-functions. This section describes such architecture, with special emphasis on describing all the TLPEs involved in the process.

The architecture described in this chapter was introduced in [127]. It is an evolution from previous works presented in $[38,45]$ and $[16,147]$. The main improvements of this new architecture are the more granular division of components and the use of formal languages to share information among these components.

For better readability, the architecture of the trajectory predictor is described using a bottom-up approach. Therefore, smaller engines are described first, which will be later combined to conform more complex engines, ultimately obtaining the trajectory predictor.

\subsubsection{Trajectory computation engine}

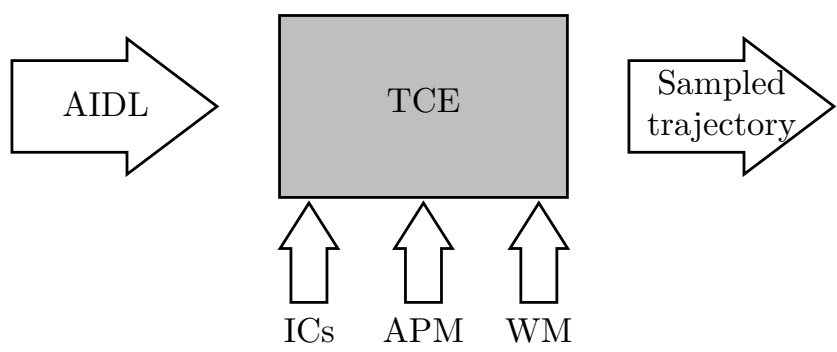

Figure 11.2: Trajectory computation engine (TCE).

A trajectory computation engine (TCE) is a translation engine that transforms an intent expressed in AIDL into a sampled trajectory [78]. This TLPE also requires the appropriate initial conditions (ICs) of the aircraft - the initial values of some of the variables in the state vector-, the aircraft performance model (APM) - containing aircraft's performance and capabilities data-, and the weather model (WM) - which includes air density, pressure and temperature variations, as well as a 4D wind prediction-, as shown in figure 11.2. This data is sufficient to describe the trajectory univocally, so the output sampled trajectory is unambiguous for any AIDL word.

The TCE computes the sampled trajectory using numerical integration of the equations of motion of the aircraft and the motion constraints imposed by the aircraft intent. Some elements specified in the AIDL may require iterative computation of the trajectory to obtain a solution: this is the case of auto triggers. Since the AIDL is built on top of these motion equations, any possible set of constraints that a TCE could process can be expressed using AIDL. Therefore, any other 'flight script' language (i.e., a language used to express the aircraft intent or behaviour model of an aircraft) can be translated into AIDL using an interpreter. Interpreters enable using AIDL as the common format for trajectory synchronization among aircraft and ground-based systems [144]. Interpreters can also be used conversely, to translate from AIDL to other 
formats, which allow using 'legacy' trajectory engines to produce a sampled trajectory [47].

\subsubsection{User preferences engine}

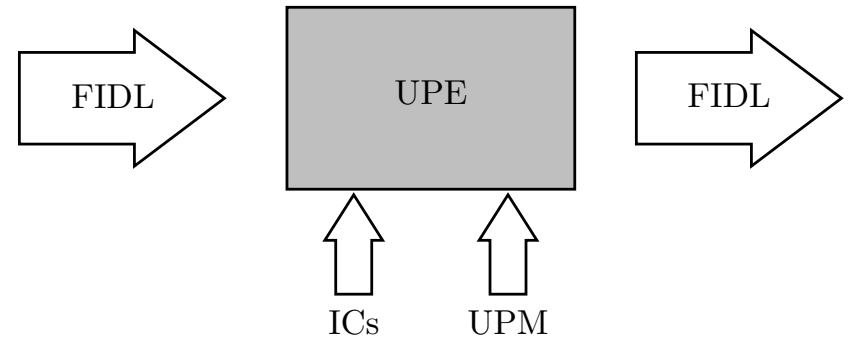

Figure 11.3: User preferences engine (UPE).

The user preferences engine (UPE) is an enrichment engine that adds user preferences (also called company preferences) to a flight intent expressed in the FIDL. These preferences are extracted from a user preferences model (UPM). The UPE also takes into account the ICs of the aircraft, as shown in figure 11.3.

The UPM contains information of three kinds: preferred routes, constraints, and objectives. Preferred routes consist of airways, SIDs, STARs, or sequences of waypoints that the airspace user wants their aircraft to follow, when available. The constraints and objectives included in the UPM are those considered by the airspace user, and they are analogous to those of the FIDL (see sections 8.1.3 and 8.1.4). Usually, airspace users will have different UPMs to choose from, depending on the type of aircraft and other circumstances.

The enrichment process performed by the UPE comprises three steps:

- The first step is the path completion using preferred routes. The UPE analyses the input flight intent and includes intermediate waypoints, airways, SIDs, or STARs. Several strategies can be followed to include preferred routes: one of them is to compute whether a preferred route can be used to complete a particular fragment of the flight. If so, the shortest preferred route (or combination of preferred routes) is used, which can be computed with a shortest-path algorithm (e.g., Dijkstra's algorithm [148, 149]). This strategy may be designed so that SIDs are only used in the initial part of the flight, and STARs are only used in the last part. The resulting flight intent will contain additional flight segments and path constraints, so that the aircraft follows the appropriate sequence of legs or airway segments.

- The next step is the addition of constraints to the flight intent. Only the constraints relevant to the flight are added. To determine which constraints are relevant, spatial filtering of their DoAs is performed. Constraints whose DoA won't intersect the trajectory (i.e., spatial DoAs far from the trajectory) are not included in the flight intent. Transcription of constraints in the UPM to FIDL constraints is straightforward.

Typical constraints added by the UPE are the preferred climb and descent speeds, and cruise altitude and speed. 
- Finally, objectives are added to the flight intent. An identical filtering process is used to determine which objectives considered relevant for the flight.

There isn't a unique strategy for enrichment of a flight intent by the UPE. In fact, several strategies may be available and several results may be generated. For example, multiple results may contain a different choice of preferred routes. Whereas the UPE initially provides only the first of those results, subsequent results can be obtained on demand.

\subsubsection{Operational context engine}

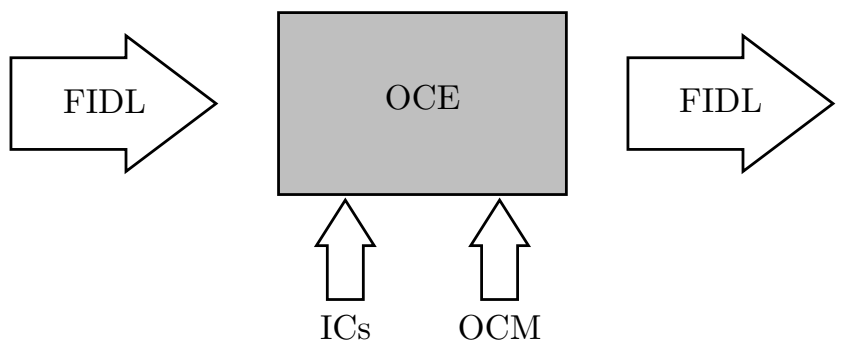

Figure 11.4: Operational context engine (OCE).

The operational context engine (OCE) is an enrichment engine that manipulates a flight intent to incorporate information from the operational context [150]. The information of the operational context is extracted from an operational context model $(\mathrm{OCM})$. In addition to the OCM, the OCE requires the ICs to perform its calculations. The input and output of this TLPE is depicted in figure 11.4.

An OCM comprises three types of elements:

- Structural elements, including airspace sectors, restricted zones, aerodromes, and runways.

- Elements related to standard procedures, such as significant points, legs, and routes (STARs, SIDs, etc.).

- Elements related to ATC constraints, including altitude and speed constraints.

There are some widely-used models to store operational context information. Among these are the AIXM [57] and the ARINC 424 specification [58].

The enrichment process carried out in the OCE is divided in two steps:

- In the route completion step, the OCE will include any missing intermediate waypoints in the flight intent (these waypoints are included as additional flight segments with a waypoint end trigger). These waypoints are obtained from the routes, SIDs, and STARs of the OCM. Path constraints are included in the added flight segments to ensure that the appropriate legs or airway segments are followed. 
Again, route completion can be performed following different strategies. One strategy may consist in finding the shortest path among all the routes in the OCM using a shortest path algorithm such as Dijkstra's [148, 149]. A different strategy may be not adding any intermediate points at all, and therefore flying directly between each couple of points in the input flight intent. This latter strategy may not respect airspace constraints, but it can be acceptable in some contexts (e.g., emergency situations).

- Constraints from the OCM that are relevant to the flight are also added to the flight intent. The filtering process that determines which constraints are relevant is based on analysing the DoAs of such constraints to see if they may intersect the trajectory. Relevant constraints are transcribed to fit the FIDL notation.

The output provided by the OCE is a flight intent with more information: it includes additional flight segments with the waypoints of the selected route, plus all the constraints deemed relevant.

As the UPE, the OCE can generate several different output, corresponding to different route completion strategies. The OCE will initially provide only one output flight intent, but alternate flight intents may be available on demand.

\subsubsection{Aircraft performance limitations engine}

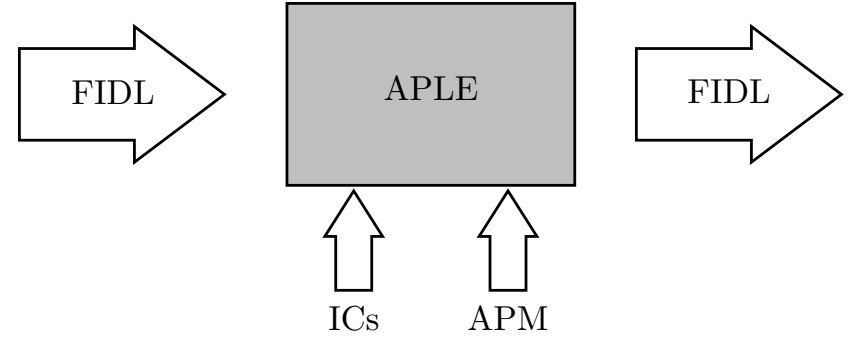

Figure 11.5: Aircraft performance limitations engine (APLE).

The aircraft performance limitations engine (APLE) is an enrichment engine taking a flight intent and adding constraints related to the aircraft performance - mainly for flight envelope protection - using the information from the aircraft performance model (APM). These constraints include the maximum operating altitude, and minimum and maximum operating speeds. As shown in figure 11.5, the APLE also takes into account the ICs.

The APLE uses the BADA $[59,151]$ to model and obtain APM information. BADA provides a model specification for aircraft performance information, plus datasets for a broad range of aircraft types.

\subsubsection{Intent generation core process engine}

The intent generation core process engine (IGCPE) is a translation engine that generates an intent expressed in AIDL compatible with the input intent 


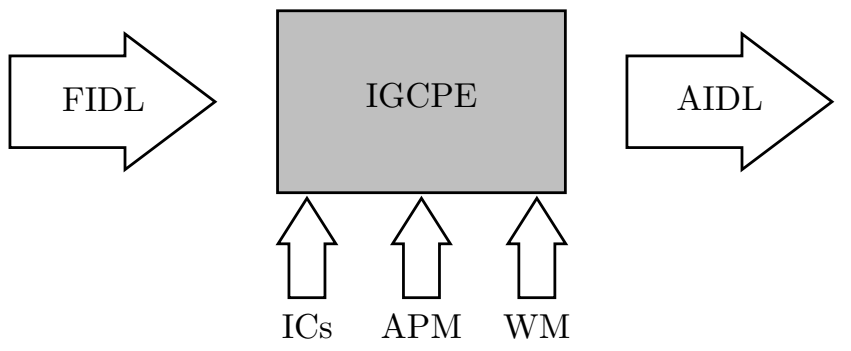

Figure 11.6: Intent generation core process engine (IGCPE).

expressed in FIDL. This TLPE (shown in figure 11.6) also requires the ICs of the aircraft to perform its calculations.

The IGCPE uses the ICDL as an intermediate representation of intent during most of the translation process, and it requires some other TLPEs:

- The TCE is required to ensure that the output intent is feasible. This output intent can be computed several times as it is being built, so decisions can be made depending on the feasibility of a partial trajectory. As a consequence of using a TCE, the IGCPE requires the information from the APM and the WM.

- The composite optimization engine (COE) is used to optimize the output according to the objectives in the input flight intent. Optimization is performed on the intermediate representation, an ICDL word, by computing the best values for all open interval and set parameters, according to the optimization criteria.

- The composite translation engine (CTE) is required to translate the intermediate representation of intent, based on the ICDL, to the output language, expressed in the AIDL. This translation is also required every time a trajectory must be computed from a composite (i.e., an ICDL word).

As some other engines described in this chapter, the IGCPE may be able to provide more than one possible output. While only the first result is provided at first (which is considered the best result according to some optimality criterion), the following results can be retrieved on demand.

The processes carried out by the IGCPE will be described in detail in chapter 12 .

\subsubsection{Intent generation engine}

The intent generation engine (IGE) is a translation engine that generates an aircraft intent from a flight intent. This engine combines the information in the flight intent with additional information from the UPM, OCM, and APM, before performing the translation. The IGE is basically a concatenation of several of the engines described before, as shown in figure 11.7. The input FIDL word is enriched using the UPE, the OCE, and the APLE, and then translated to 


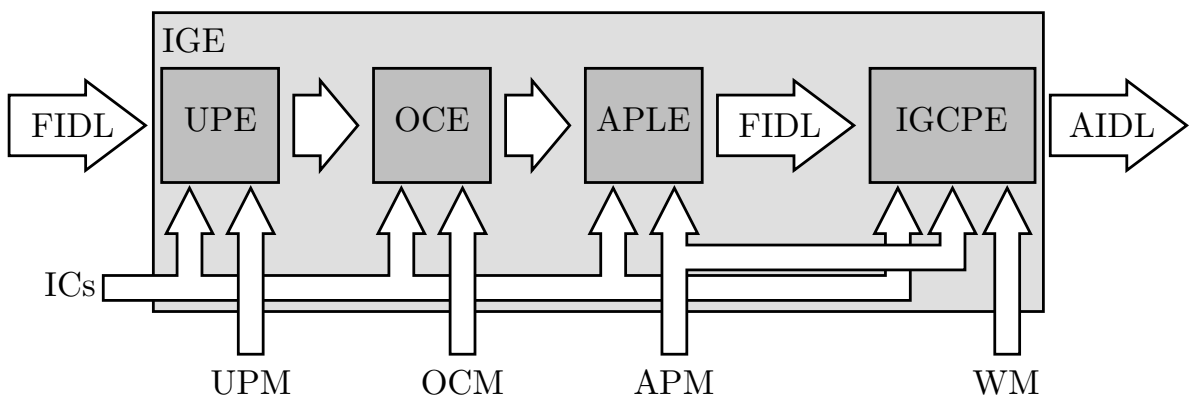

Figure 11.7: Intent generation engine (IGE).

an AIDL word using the IGCPE. Therefore, in addition to the aforementioned models, the ICs and the WM are also required to complete the translation.

Given the flight intent resulting from the enrichment by the UPE, OCE, and APLE, it may be not possible for the IGCPE to compute a feasible aircraft intent. In this case, as the UPE and OCE may provide more than one possible solution, all the available solutions are used, until one of those solutions can be successfully translated into an aircraft intent.

\subsubsection{Trajectory prediction engine}

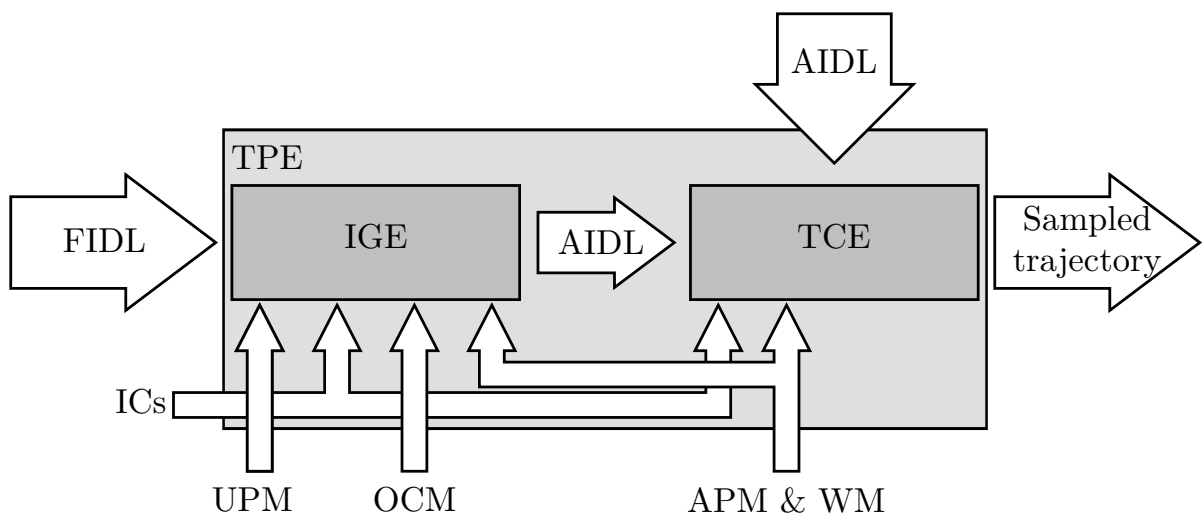

Figure 11.8: Trajectory prediction engine (TPE).

The trajectory prediction engine (TPE) is a translation engine that computes a sampled trajectory from either a flight intent (expressed in the FIDL) or an aircraft intent (expressed in the AIDL). This engine is a combination of two engines previously described: the TCE and the IGE (see figure 11.8). If the input is an aircraft intent, then the TCE is used to compute the sampled trajectory. Otherwise, the IGE is used to translate the flight intent into an aircraft intent that can, in turn, be translated into a sampled trajectory. In any case, the TPE needs the ICs, the APM and the WM. Additionally, if the input is a flight intent, the UPM and OCM are required. 


\subsection{Examples}

The architecture described in this chapter is complemented with an example showing the functionality of several engines. This example shows the evolution of a flight intent during the enrichment process in the TPE, and the posterior computation of a sampled trajectory. A similar example was provided in [127], but it has been updated to reflect the changes and improvements made since. Details on the input and output of every intermediate step are provided.

The TMA of the 'Las Palmas de Gran Canaria' airport (GCLP, in ICAO notation) has been chosen as the scenario for the example. The TPE will be used to predict an area navigation (RNAV) approach to such airport by an aircraft (a Boeing 737-800), which is initially in cruise phase. The flight intent provided to the TPE contains only one flight segment, which indicates the final destination of the flight. In the notation in appendix D, this flight intent is written:

\section{FLIGHT SEGMENTS $(\langle$ GCLP $\rangle)$}

Since the TPE is provided with a flight intent, it will use the IGE to translate it to an aircraft intent. The process carried out in the IGE starts with the enrichment of the flight intent using the airspace user's preferences. In this particular example, the UPE will choose a preferred path from the UPM: a STAR named TERTO6C. This STAR describes the path to be followed in the segment between points TERTO and ENETA. The latter point is the initial approach fix (IAF). These points are added to the flight intent, together with a path constraint indicating the STAR to be followed. Constraints indicating the preferred descent speed are also included. These constraint has a priority of zero so it doesn't interfere with other constraints, which would have preference if incompatibilities existed.

The UPE will finally add the user's optimization criteria in the form of one or more objectives. In this case, the UPE is configured to optimize flight duration, and therefore an objective is added with the purpose of minimizing flight time (i.e., minimize $\Delta t=\int_{\Omega} 1 \mathrm{~d} t$ ). Therefore, the output from the UPE is the following flight intent:

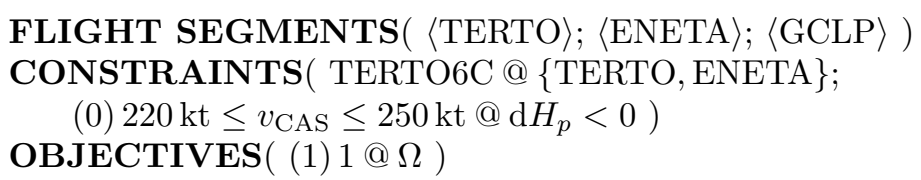

Other UPE configurations could be used to define alternate objectives for optimizing the flight.

This flight intent is passed to the OCE, which will add any relevant information from the OCM to it. In this case, the path is completed by adding the intermediate points of the TERTO6C STAR (see figure 11.9) and the corresponding approach from the IAF, ENETA, to the runway (see figure 11.10). Also, all the constraints from the OCM that may affect the flight are included. The result of the OCE enrichment is: 


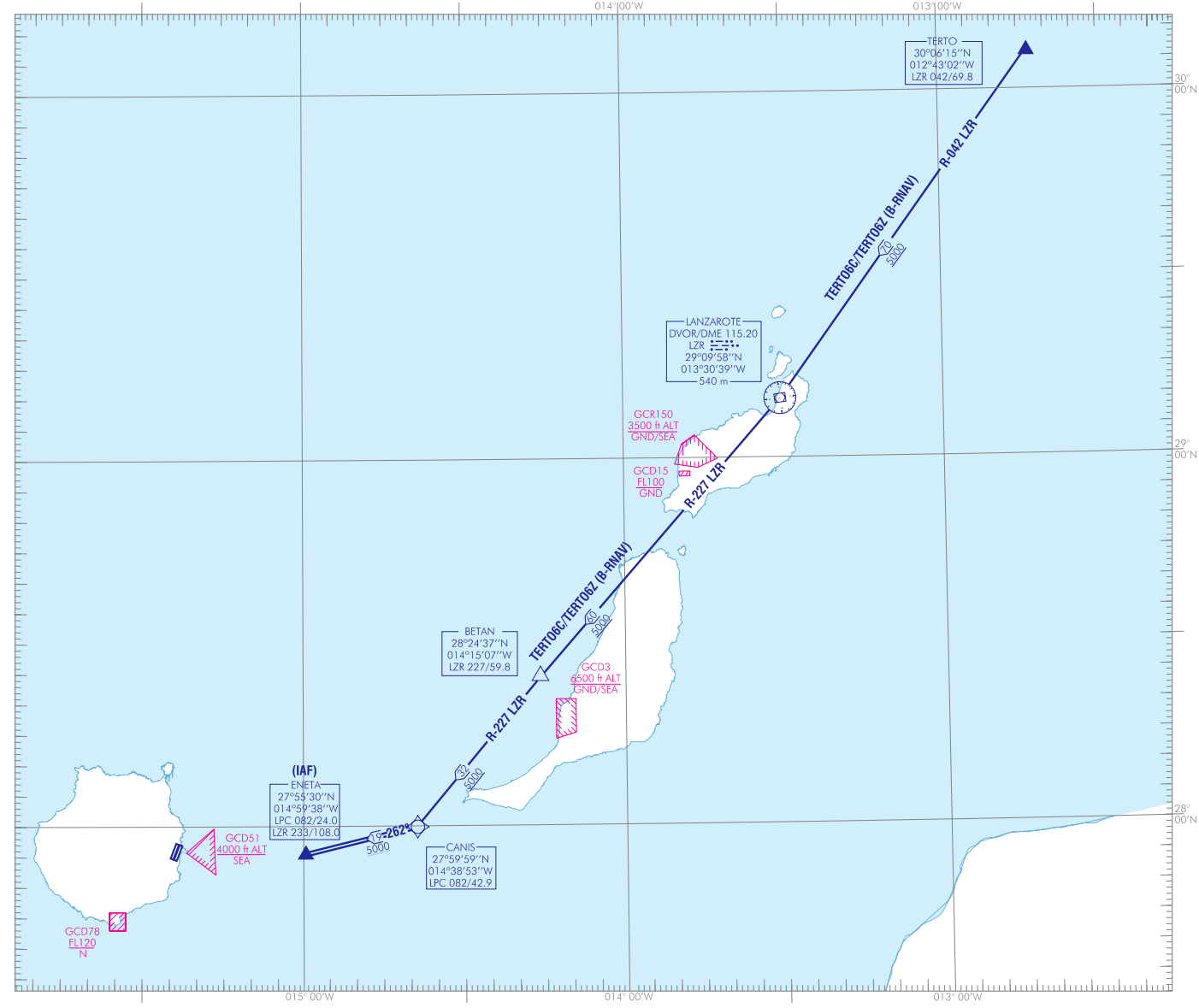

Figure 11.9: TERTO6C STAR and relevant constraints. 


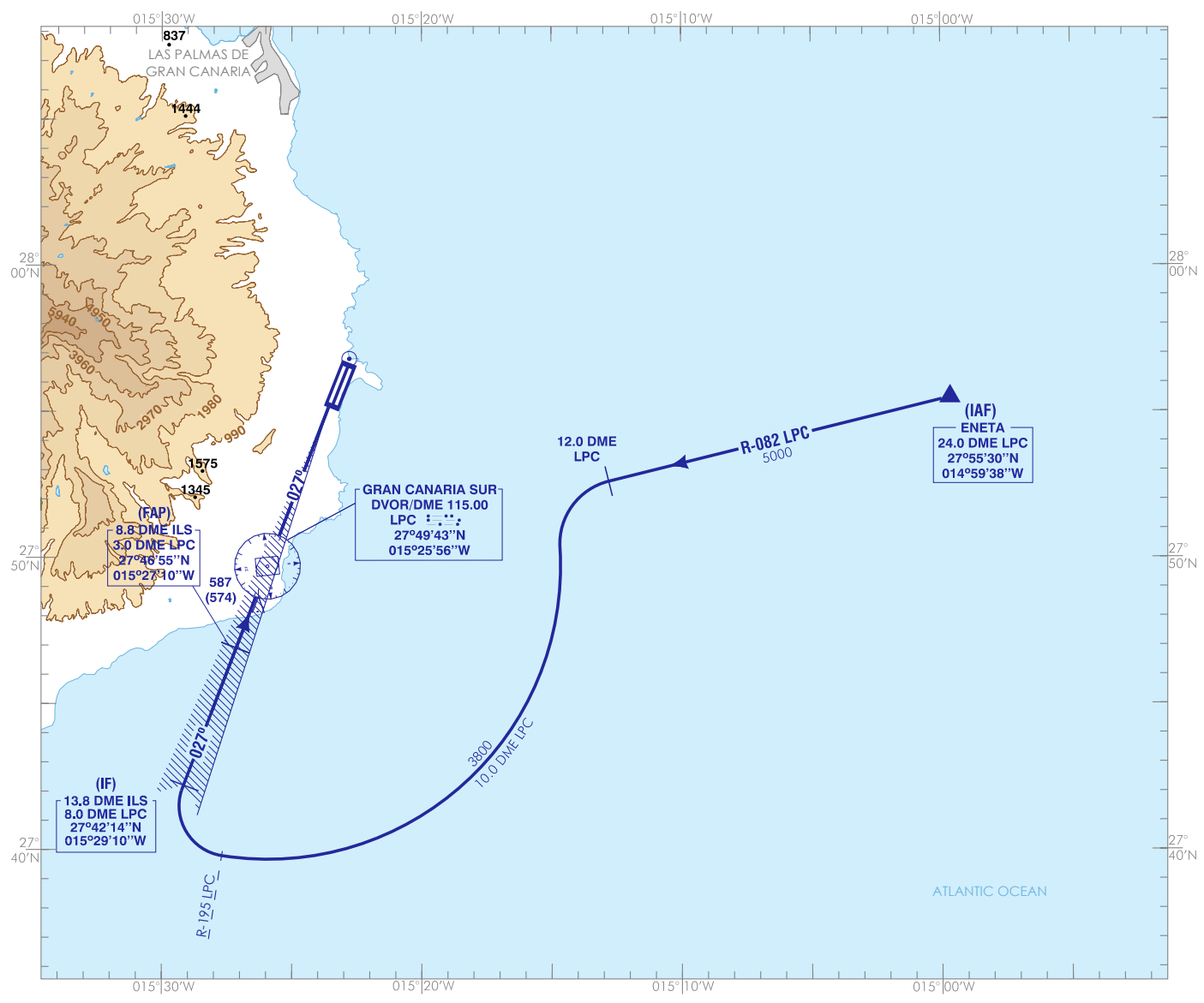

Figure 11.10: Details of the approach from ENETA to GCLP airport. 


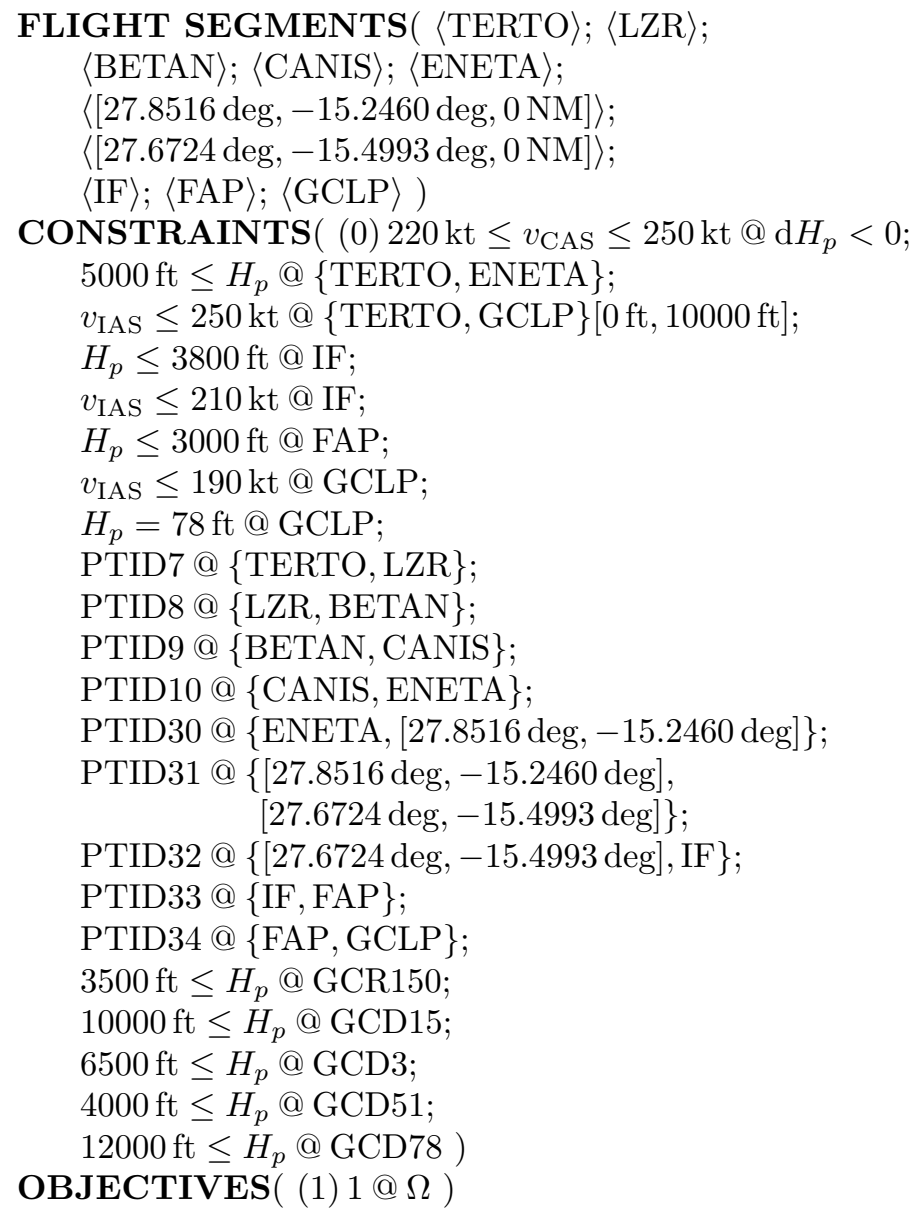

In this flight intent, new flight segments with waypoint end triggers are added. The flight segments ending at LZR, BETAN, and CANIS correspond to the intermediate points of the TERTO6C STAR, shown in figure 11.9. After ENETA, the flight segments of the instrument approach (which is outlined in figure 11.10) are added. This approach starts with two waypoints that are added as pairs of coordinates (latitude, longitude). The first of them is the intersection of the circle defined by the DME '10.0 DME LPC' and the VOR radial 'R-082 LPC'. The aircraft will fly along the leg PTID31 (an arc of the '10.0 DME LPC' circle) until the next point, which is the intersection of the same circle with the VOR radial 'R-207 LPC'. The next two points are the intermediate fix (IF) and the final approach point (FAP), to finally reach the airport.

The OCE will also add a number of constraints. The first constraint corresponds to the TERTO6C STAR, where altitude must be above 5000 feet. The second constraint is the TMA speed limit, 250 knots under 10000 feet. The next 5 constraints are related to the approach procedure. Also, path constraints corresponding to the legs of the STAR (PTID7 to PTID10) and the approach (PTID30 to PTID34) are included. Finally, some altitude constraints defined over a volume DoA are included. These DoAs are included in the flight intent by name, but their actual locations are shown in figure 11.9. 
Then, it is turn for the APLE to add the constraints related to the aircraft performance. The flight intent resulting from the APLE enrichment is:

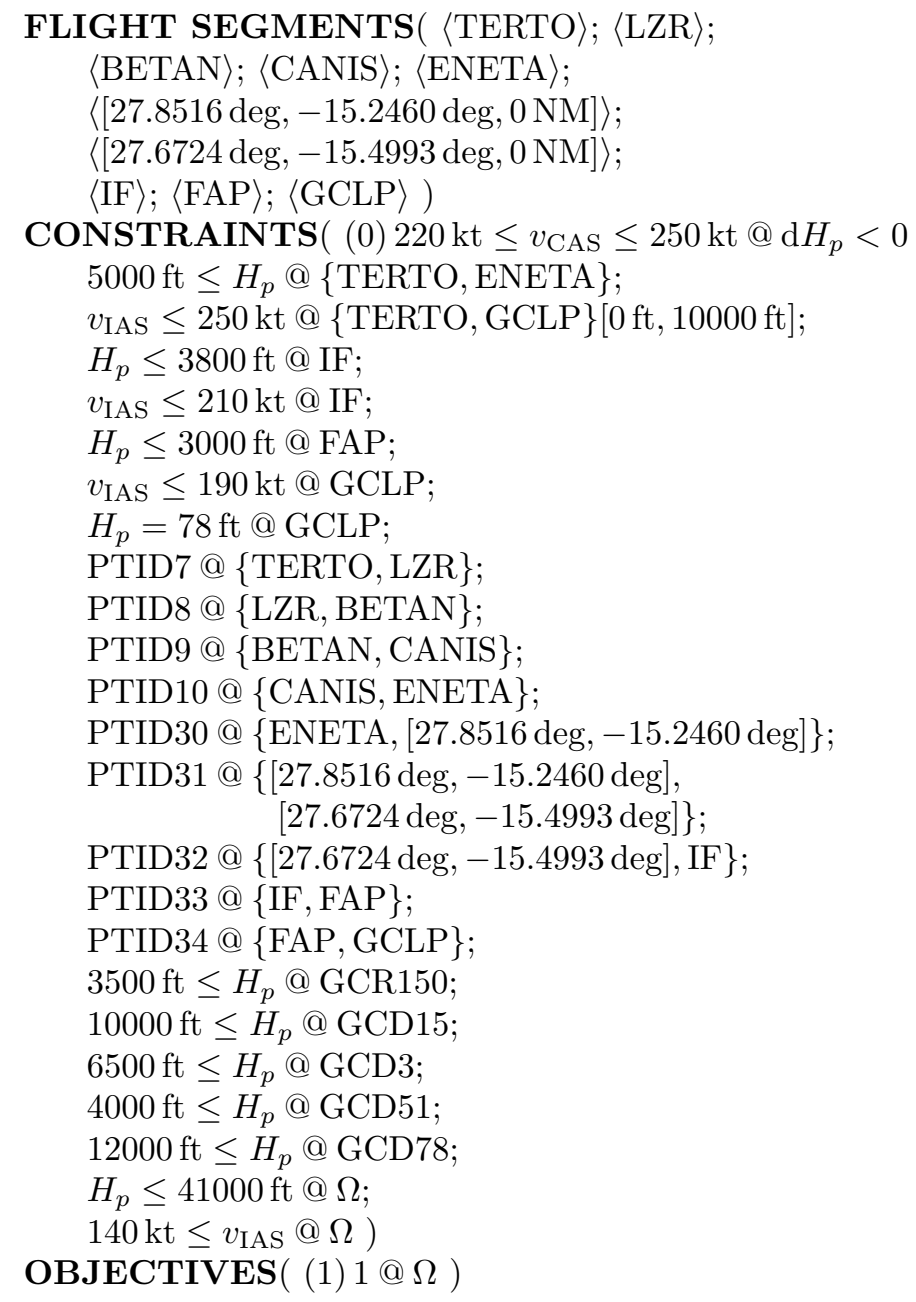

Two new constraints are appended at the end of the constraint list: the service ceiling (i.e., the maximum operating altitude), and the minimum speed (with flaps fully deployed).

When the enrichment process finishes (after performing the enrichment by the UPE, OCE, and APLE), the only step remaining is the translation of the enriched flight intent into an aircraft intent. This translation is performed by the IGCPE. Note that the flight intent above refers to some elements of the OCM - waypoints and volume DoAs - by name, to improve readability. However, the IGCPE doesn't have access to the UPM or OCM, and therefore all the necessary information from any of these models should be embedded in the enriched flight intent.

The IGCPE produces an aircraft intent, which becomes, by extension, the output of the IGE. Since this translation is not univocal, there are many possible translations. One possible translation is the AIDL word in figure 11.11. 


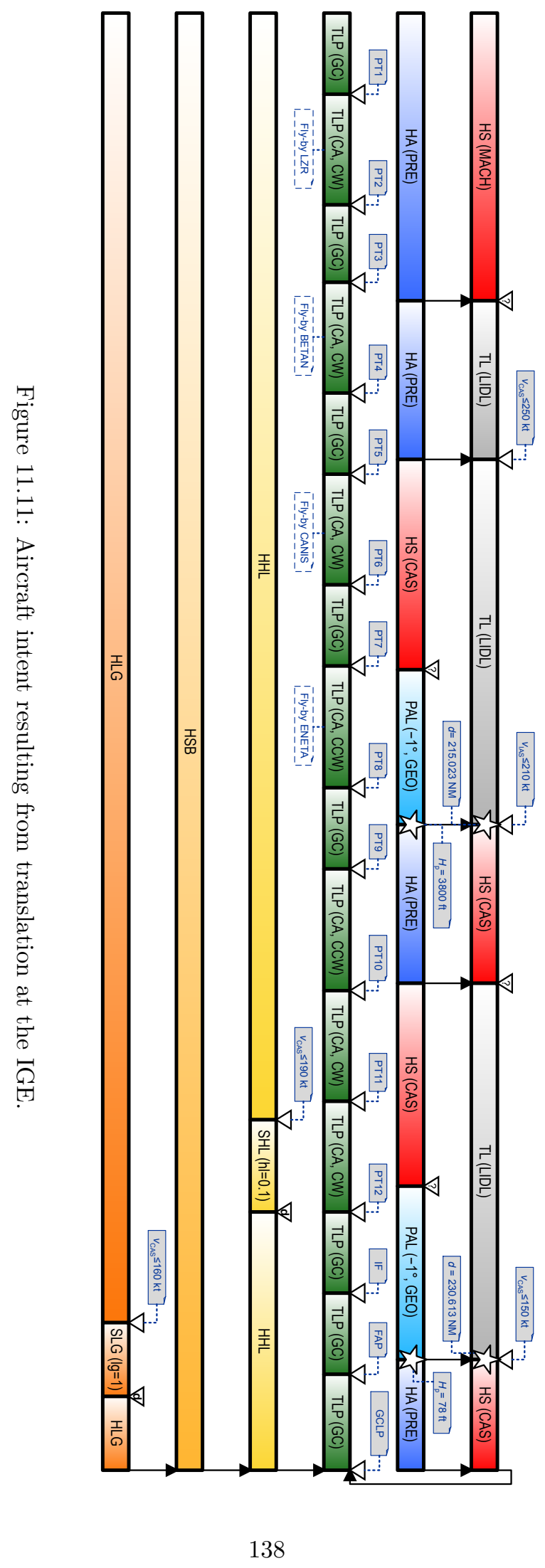


This aircraft intent starts with a level flight until the top of descent (TOD), defined using an auto trigger. The aircraft will then proceed to decelerate until it reaches the preferred descent speed, to later start an idle thrust (LIDL) descent. The descent has two stages: during the first stage, the aircraft descends with constant CAS, whereas in the second phase, a constant path angle of 1 degree is used, which reduces the speed. The auto triggers ensure that the target altitude of 3800 feet and speed of 210 knots are reached simultaneously just before the aircraft reaches the IF. An identical two-phased idle descent is performed to reach the desired altitude and speed immediately before landing. In the lateral

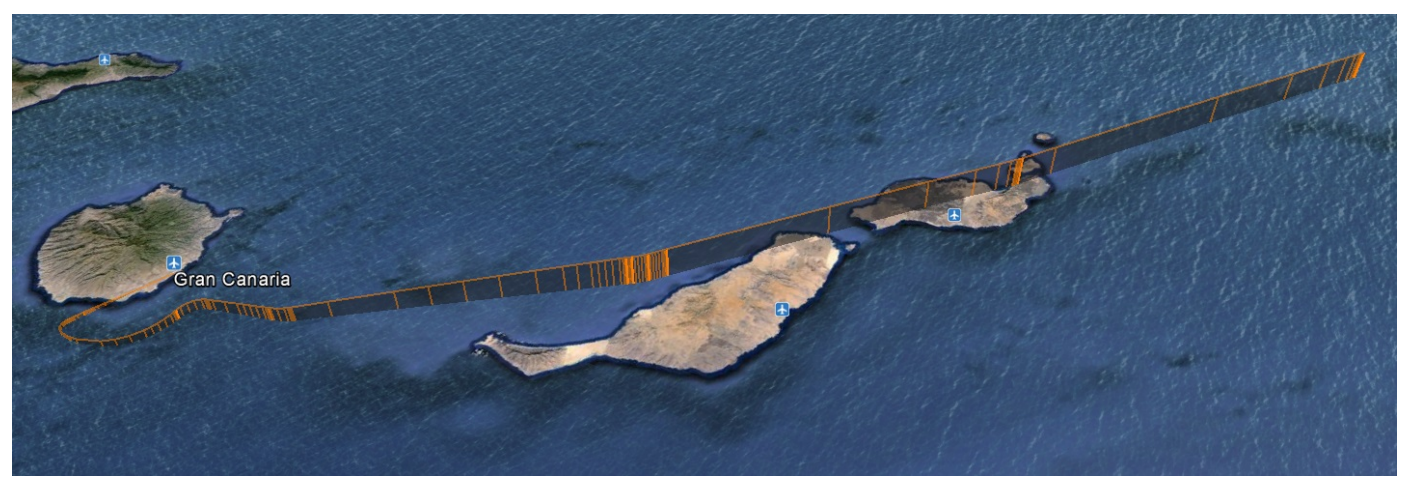

Figure 11.12: Trajectory generated from the resulting aircraft intent (Map data: Google, Landsat, Europa Technologies).

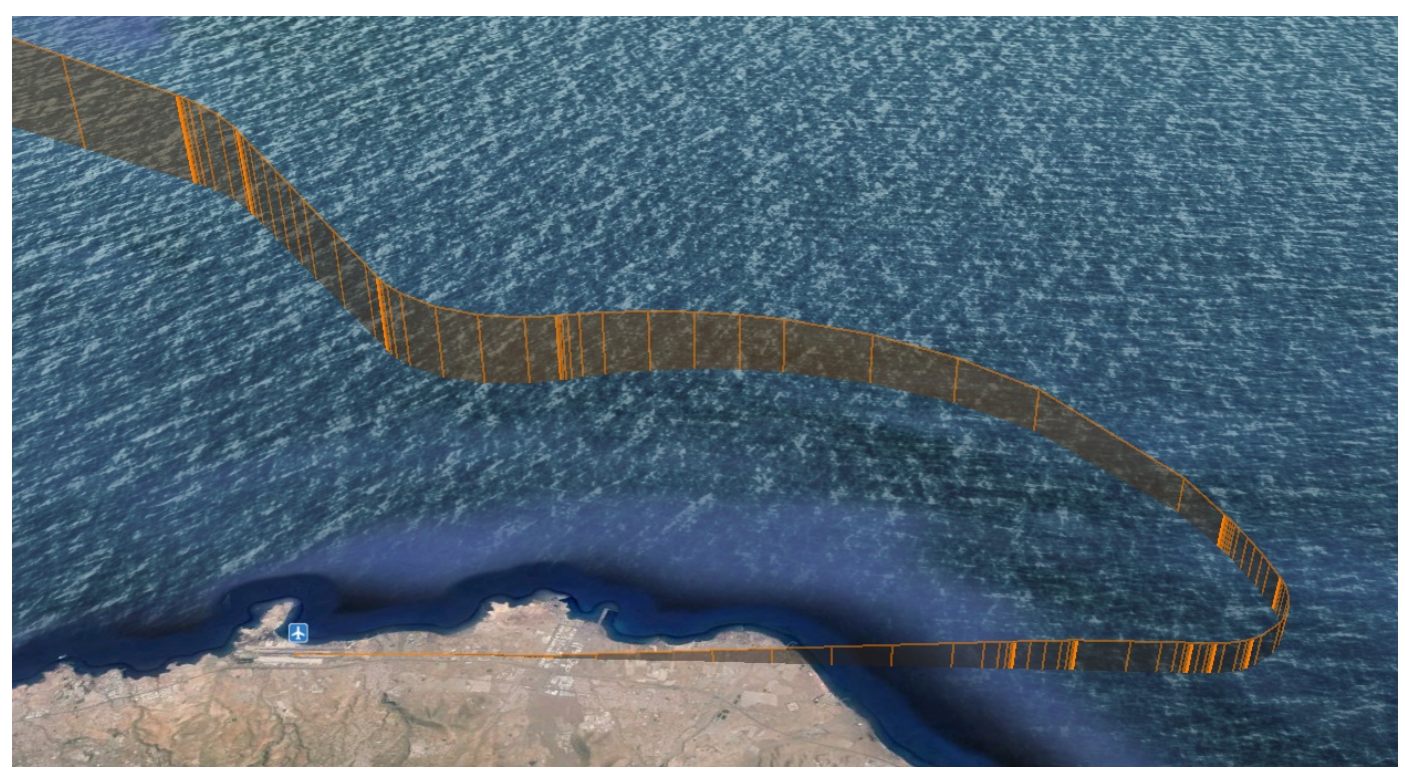

Figure 11.13: Detail of the approach manoeuvre (Map data: Google, TerraMetrics, GRAFCAN, Europa Technologies). 


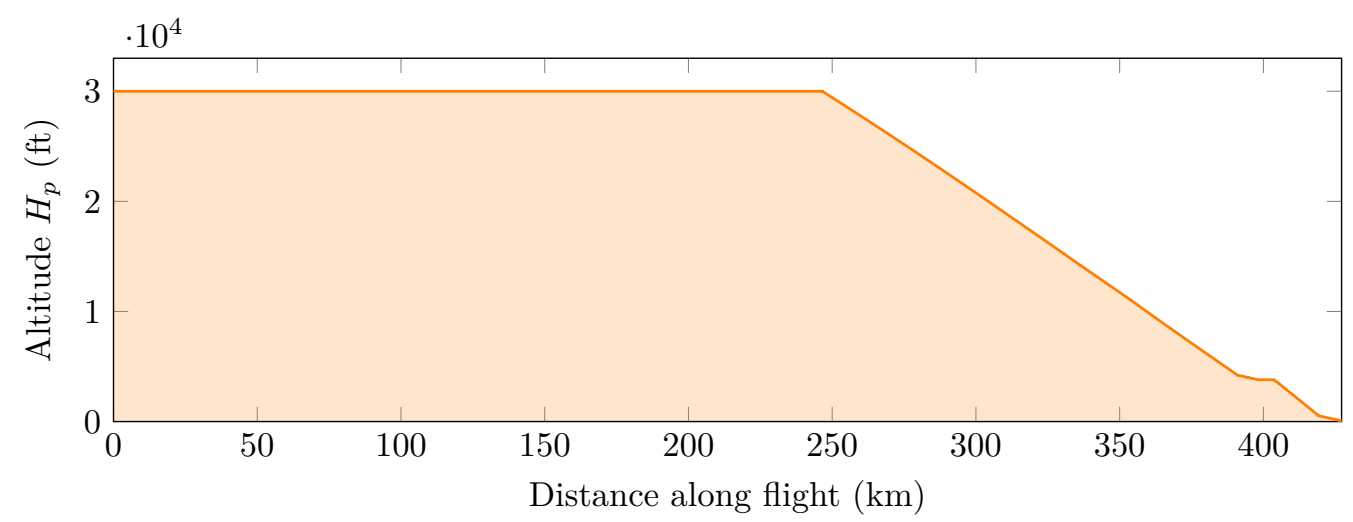

(a) Barometric altitude of trajectory along distance.

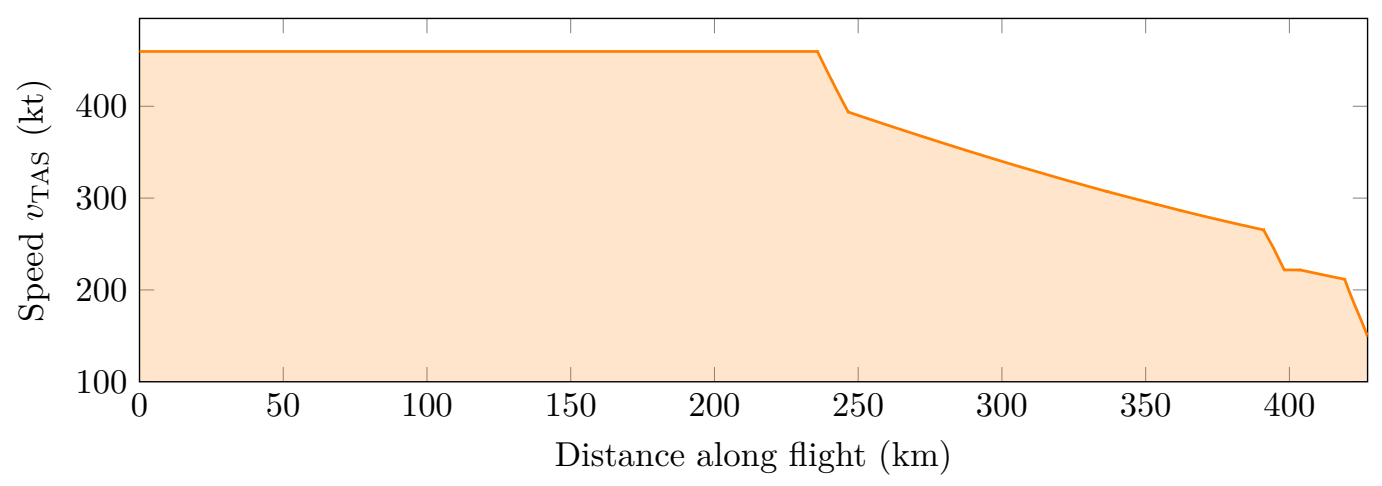

(b) TAS of trajectory along distance.

Figure 11.14: Altitude and speed of the approach to GCLP.

profile, a concatenation of great circles and circular arcs is used. During the translation process, some auxiliary points (PT1-PT12) are computed. These points indicate the start and end of turns in fly-by manoeuvres. In the configuration profiles, speeds are used to trigger landing gear and high lift (flaps) deployment.

With the appropriate WM and APM, the TPE can translate the aircraft intent in figure 11.11 into a sampled trajectory using the TCE, which is depicted in figure 11.12. The evolution over time of the aircraft's altitude (figure 11.14a) and speed (figure 11.14b) is also provided. A close look at the final part of the trajectory, corresponding to the instrument approach manoeuvre, is shown in 11.13. This trajectory is the final result of the process carried out by the TPE.

\subsection{Final words}

This chapter has presented a modular decomposition of a trajectory predictor using the concept of TLPEs, or engines. This decomposition is more fine-grained than previous trajectory prediction structures $[16,38,45,147]$, although it is 
compatible with such structures.

The engines that compose the trajectory prediction communicate in terms of the languages described in part II of this thesis, particularly the FIDL and the AIDL. The communication using those languages has applications beyond trajectory prediction. Operational DSTs, such as arrival managers, FMSs, flight data processing systems, air-ground synchronization, etc., could also benefit from the concept of TLPEs and their associated languages.

The modular approach to building a trajectory predictor provides additional benefits. It allows for better scalability, as the trajectory prediction process can be pipelined to improve throughput via parallel processing. It also improves maintainability, as engines can be developed, tested, and improved in isolation. Drop-in replacements to some engines can be developed while maintaining the existing structure. New features can also be added to the IGE by adding a new engine to the pipeline.

Functional descriptions of the engines have been provided together with examples showcasing that functionality, but details on the algorithms used have not been included. In the next chapter, more details on one of the key engines in the trajectory predictor - that is, the IGCPE - are provided, including descriptions of the algorithms and design used by this engine.

The engines presented in this chapter were designed for ATM, but the same ideas can be extrapolated to other environments, such as UAS. A similar decomposition of the trajectory prediction problem could be developed for that environment, obtaining an analogous functionality. For example, a TCE for computing trajectories of quadrotor aircraft will be described in chapter 13 . 



\section{Chapter 12}

\section{The core of the intent generation process}

In the last chapter, the intent generation core process engine (IGCPE) was presented. This engine is responsible for translating an enriched flight intent (that is, a flight intent containing all the relevant data from the UPM, OCM, and APLE) into an aircraft intent. The IGCPE is the most important and complex engine composing the IGE, yet very little information has been provided on how this translation process works.

This chapter provides detailed information on the implementation of the IGCPE, including the algorithms used to generate the horizontal and vertical profiles. In chapter 3 , some of the previously existing methods were described, most of which rely on pre-defined profiles that are later adapted to the constraints of the particular flight [60, 83, 86, 87]. The IGCPE uses a different approach to the generation of the vertical profile. This approach, developed in collaboration with BR\&TE, was inspired by search strategies used in artificial intelligence [152]: a decision tree is generated to evaluate possible manoeuvres that comply with the flight's constraints. This novel approach provides more flexibility, as the decision tree can be expanded using many different strategies - denoted micro-strategies - , each of them addressing constraints using different manoeuvres. The approach is complemented with a modular design, allowing to easily replace components of the IGCPE to fit the needs of most applications of trajectory prediction.

The next section provides a general description of the process carried out at the IGCPE. The two sections after that describe the algorithms that generate the horizontal and vertical profile, respectively. Finally, an example is shown to provide qualitative and quantitative results.

\subsection{Process description}

The main task of the IGCPE is to translate a flight intent into an aircraft intent. The most suitable language to express trajectories during most of the required computations is neither the FIDL — oriented towards description of 


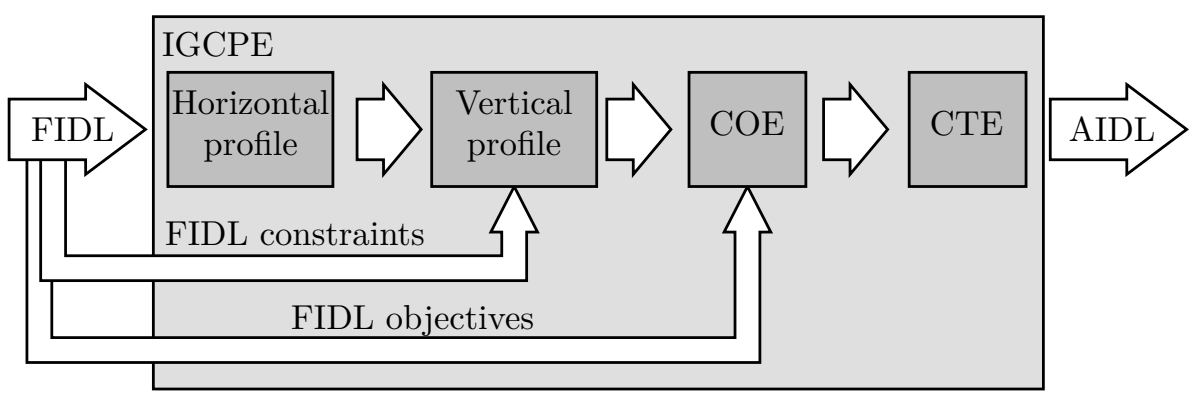

Figure 12.1: Intent generation core process.

flight requirements - nor the AIDL — too constrained and requesting definition of all movement profiles at the same time-, but the ICDL. The reason is that the ICDL enables the definition of pilot manoeuvres independently for different profiles, and leaves room for later refinement of parameters or parts of the trajectory. So, it is very appropriate for the incremental definition of the trajectory. Therefore, the translation process that takes place in the IGCPE can be divided in several sub-processes, as shown in figure 12.1. The translation process starts with the generation of a composite that is compatible with the input flight intent. This is performed in two steps: first the horizontal profile is generated; then, the vertical profile. Finally, the generated composite is optimized and translated into AIDL. Exchange of flight information between processes inside the IGCPE always uses the ICDL.

Composite generation is a complex task, as much critical information is added to the intent in this phase. Most important decisions, such as the manoeuvres that will be used to comply with each constraint, are taken at this point. In the proposed IGCPE design, the generation of the composite is divided in two steps. First, a geometrical horizontal profile is generated according to the information present in the flight intent. Then, the vertical profile is computed $^{1}$, combining the information in the horizontal profile and the flight intent to find the constraints affecting the flight.

The composite generated in that phase can be optimized by tweaking some of its numeric parameters, such as speeds, altitudes or thrust profiles. The ICDL includes means to indicate what parameters can be optimized, and what are the appropriate intervals in which the optimal should be found. In the optimization phase of the IGCPE, the composite's parameters are optimized according to the objectives present in the input flight intent. These objectives (i.e., a series of weighted cost functions) can refer to fuel consumption, flight time, emissions, comfort, etc. In the example provided in section 12.4, a combination of fuel- and time-related costs is used. The optimization phase can be seen as another independent enrichment engine, the composite optimization engine (COE), where both the input and output are expressed in ICDL. The COE closes the open parameters of the input composite by choosing the optimal — or near-optimal - values (i.e., the optimal magnitude for each interval parameter,

\footnotetext{
${ }^{1}$ The vertical profile is tightly coupled with the configuration profile (i.e., flaps, slats, and landing gear situation), and therefore the two are generated simultaneously. However details on the configuration profile generation have been omitted in this chapter.
} 
and the optimal specifier for each set parameter). The output of this TLPE is another composite with no open interval or set parameters. Many different methods can be used to optimize a composite according to certain objectives. In the proposed implementation, the COE uses a non-deterministic optimization method, known as the complex method [153], to obtain the optimal values for the intervals of the composite.

Once an optimized composite has been obtained, the only remaining task is to translate this composite to an aircraft intent. The translation from the ICDL into the AIDL is carried out by the composite translation engine (CTE). The composite provided to the CTE must be unambiguous, i.e., a composite defining all the execution threads with no open interval or set parameters. The optimized composite meets these criteria, as the generated composite defines all threads and all open intervals have been closed during the optimization phase. The translation carried out by the CTE is trivial when the ICDL word contains no conditional branches or loops. Otherwise, the trajectory must be computed to evaluate the conditions in the trajectory and obtain the AIDL instructions that are actually executed (as the AIDL doesn't support branches or loops). Thus, the APM and WM are required to perform the translation in this case.

\subsection{Horizontal Profile Generation}

The horizontal profile generation is the first step of the composite generation phase in the IGCPE. The horizontal profile (also called lateral path) is generated in this step by means of lateral composites. These lateral composites are chosen from different possible lateral manoeuvres or geometries that can be flown by an aircraft. In the IGCPE prototype presented in this chapter, great circles (orthodromes) or small circles (circular arcs) are the only lateral geometries (i.e., composites) used to generate the horizontal profile. This decision on which composites can be used to define the lateral path imposes restrictions to the input flight intent: the start and end of all flight segments must be defined as $2 \mathrm{D}$ points.

Some flight segments of the flight intent may already have their lateral path explicitly defined by a composite. These sections of the lateral path are preserved in the output. The remaining flight segments are then defined by their start and end points, which may be accompanied by a path constraint indicating the geometry of the horizontal profile in that flight segment. When no constraints are imposed, the algorithm will generate the shortest path, that is, a great circle. An example input flight intent is shown in figure 12.2a, where some flight segments are defined using a composite (flight segments 1 and 7, represented as a continuous line describing the horizontal profile, which must be preserved in the output), one has a great circle constraint (flight segment 5 , represented as a dashed line with the desired geometry), one has a small circle constraint (flight segment 6, also represented as a dashed line) and the remaining have no path constraints.

The main task of the horizontal profile generation is to determine how the transitions between flight segments are made using the available lateral composites. As a result of describing the horizontal profile using orthodromes and circular arcs, those transitions are simplified to just decide how the aircraft 


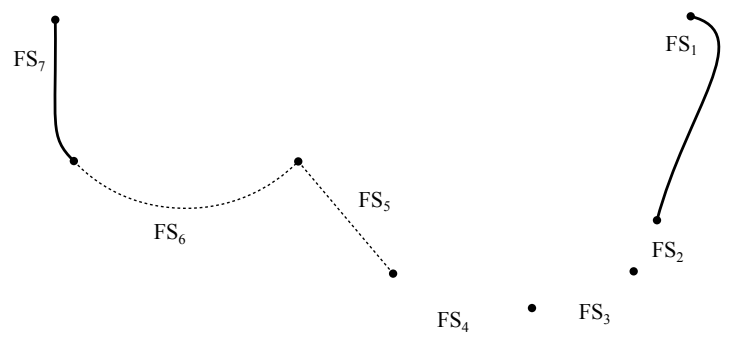

(a)

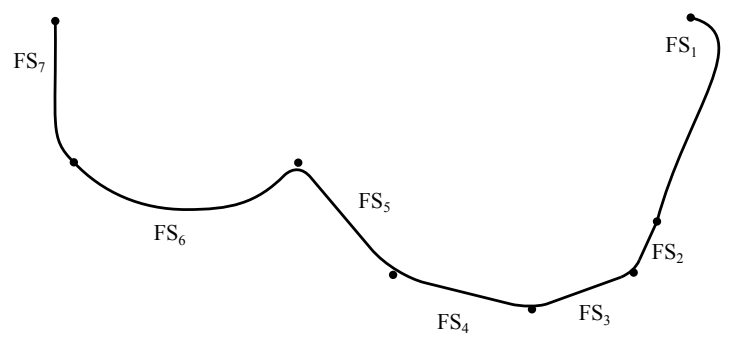

(b)

Figure 12.2: Example input and output of the horizontal profile generation.

should turn at each point. This is a two phase decision process. In the first phase, the algorithm considers each turn individually, whereas in the second phase, interactions (and possible conflicts) between turns are identified and solved. At the end, the turn at each point depends on four factors:

- The estimated speed of the flight at that point. Although the vertical profile of the flight hasn't been generated, estimate speeds are computed based on the applicable constraints in the flight intent (those relevant for the flight segments under analysis).

- The maximum allowed bank angle, which can be retrieved from the APM or bank angle constraints. Using the maximum bank angle $\theta$ and the estimated speed $v$, the minimum turn radius $R$ can be computed using the following formula [154]:

$$
R=\frac{v^{2}}{g \tan \theta}
$$

where $g$ is the acceleration due to gravity.

- The geometry of the ending and starting flight segments, including the type of legs, the angle of turn (the difference between initial and final bearing), and the distance to the next and previous points.

- The tolerance of the turning points. The tolerance is the maximum distance a lateral path can be from one particular point of the route. Fly-over points shall have a tolerance of zero. Tolerances for points of the route may be specified in the flight intent, or else a default tolerance is used. 


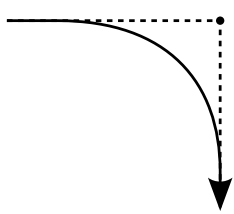

(a)

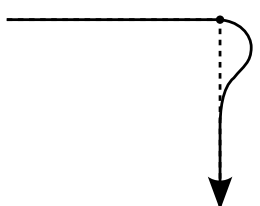

(b)

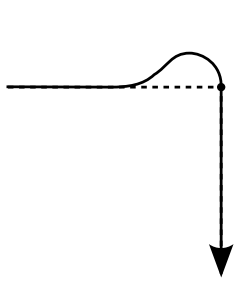

(c)

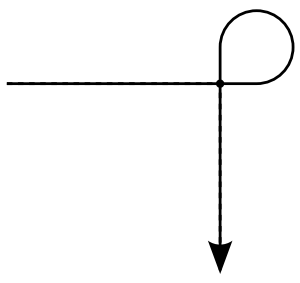

(d)

Figure 12.3: Fly-by (a), start-at waypoint fly-over (b), end-at waypoint fly-over (c), and lace (d) manoeuvres to turn at a point.

One possible output for the example input described before is shown in figure 12.2b. In this example output, all turns consist of fly-by manoeuvres with varying turn radii (depending on the estimated speed at each point). A fly-by is the preferred manoeuvre and it will be used in most cases. However, when a fly-by manoeuvre is not feasible, three other manoeuvres are considered, which are summarized in figure 12.3 .

\subsection{Vertical Profile Generation}

The vertical profile generation is the second step of the composite generation in the IGCPE. The output of the vertical profile generation is a composite that completely specifies the aircraft's behaviour. This step requires some preprocessing to express the input in a more convenient manner. After the preprocessing is completed, a search algorithm is used to find a suitable sequence of manoeuvres that meets the constraints.

The objective of the pre-processing is to transform constraints' domains of application (DoAs) into a list of pairs $\left(d_{\text {start }}, d_{\text {end }}\right)$, where $d_{\text {start }}$ is the distance (along the lateral path) at which the constraint becomes active, and $d_{\text {end }}$, the one at which it stops being active. This transformation uses the lateral path computed in the horizontal profile generation. Every DoA defined as a geometrical space (such as a point, a segment or a volume) is transformed into one or more pairs of distances. Note one DoA can be transformed into several pairs of distances if the lateral path enters and leaves the DoA more than once. DoAs that do not consist of a geometrical space are assigned a pair of distances that enclose the whole flight. Additional information of the DoA, such as altitudes or vertical speeds at which the constraint is active, is also kept.

In the implemented prototype, transformed constraints are then grouped in six categories intimately related with most typical ATM constraints: strong altitude, strong speed, weak altitude, weak speed, ascent speed, and descent speed. More categories could be added to meet new needs. The strong altitude category contains all constraints with a geometrically-defined strong DoA and an effect that limits the altitude of the flight. Equivalently, there is a speed category with all the constraints with a geometrically-defined strong DoA and an effect limiting the speed of the flight. Constraints in these two categories are sorted and merged (as shown in figure 12.4$)$ so that no pairs of distances $\left(d_{\text {start }}, d_{\text {end }}\right)$ 


\begin{tabular}{|c|c|c|c|}
\hline$H_{p}<3,000 \mathrm{ft}$ & & \multicolumn{2}{|c|}{$5000 \mathrm{ft}<H_{p}$} \\
\hline \multicolumn{3}{|c|}{$H_{p}<10000 \mathrm{ft}$} & \\
\hline$H_{p}<3000 \mathrm{ft}$ & $H p<10000 \mathrm{ft}$ & $5000 \mathrm{ft}<H_{p}<10000 \mathrm{ft}$ & $5000 \mathrm{ft}<H_{p}$ \\
\hline
\end{tabular}

Figure 12.4: Merging of altitude constraints.

of two constraints of the same category overlap. The weak altitude and weak speed categories are analogous to their corresponding strong categories, but contain the constraints with geometrically-defined weak DoAs. Ascent and descent speed constraints limit the speed of the flight while the vertical speed is above/below some threshold. For example, they allow defining a minimum CAS of 240 knots (the effect of the constraint, denoted $v_{\mathrm{CAS}} \geq 240 \mathrm{kt}$ ) when the vertical speed is above one meter per second (the DoA of the constraint, denoted $\left.\mathrm{d} H_{p} / \mathrm{d} t>1 \mathrm{~m} / \mathrm{s}\right)$.

Prior to describing the algorithm used in the vertical profile generation, some of its structures will be defined. These structures are contexts and the context collection. A context contains all the relevant information for a particular state of the vertical profile generation. This includes all the constraints and their categories, whether each constraint has been addressed (resolved constraints) or not (unresolved constraints), a composite containing manoeuvres that address resolved constraints, a partial computed trajectory resulting from such composite, and a score. The score contains a merit (or cost) value that quantifies how 'good' (or 'bad') this context is. An example context is shown in figure 12.5. The context collection is used for storing contexts and retrieving the context with the highest merit value (or lowest cost value) in an efficient manner. This

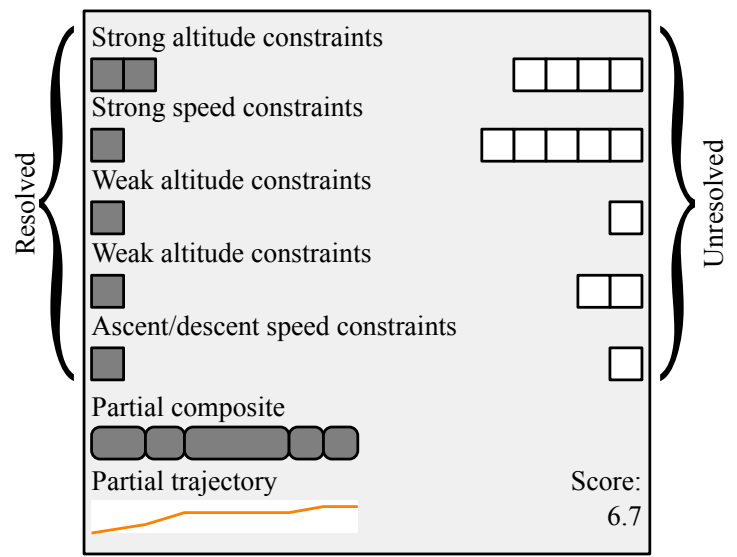

Figure 12.5: Example context. 
type of structure can be provided by a priority queue, and its most efficient implementation is the Fibonacci heap [155].

The algorithm used to generate the vertical profile builds a composite (and its corresponding trajectory) in several steps. Starting with the initial context, which contains an empty composite (i.e., one with no instructions), contexts are expanded several times. During the context expansion, a small number of constraints are addressed and the composite is altered (if necessary) to address such constraints. Contexts are expanded repeatedly until all constraints have been addressed.

Very often, there is more than one possible way of expanding a context: different constraints may be addressed, or different manoeuvres may be used to address the same constraints. The proposed algorithm is capable of handling different context expansions by creating a decision tree for exploring the search space. The context collection is used to efficiently manage the decision tree. The explored search space comprises all the sequences of manoeuvres (feasible or not) that address all the constraints.

A detailed description of the algorithm outlined above is provided next. The steps enumerated below correspond to those in figure 12.6.

1. The first step is the generation of the initial context. In the initial con-

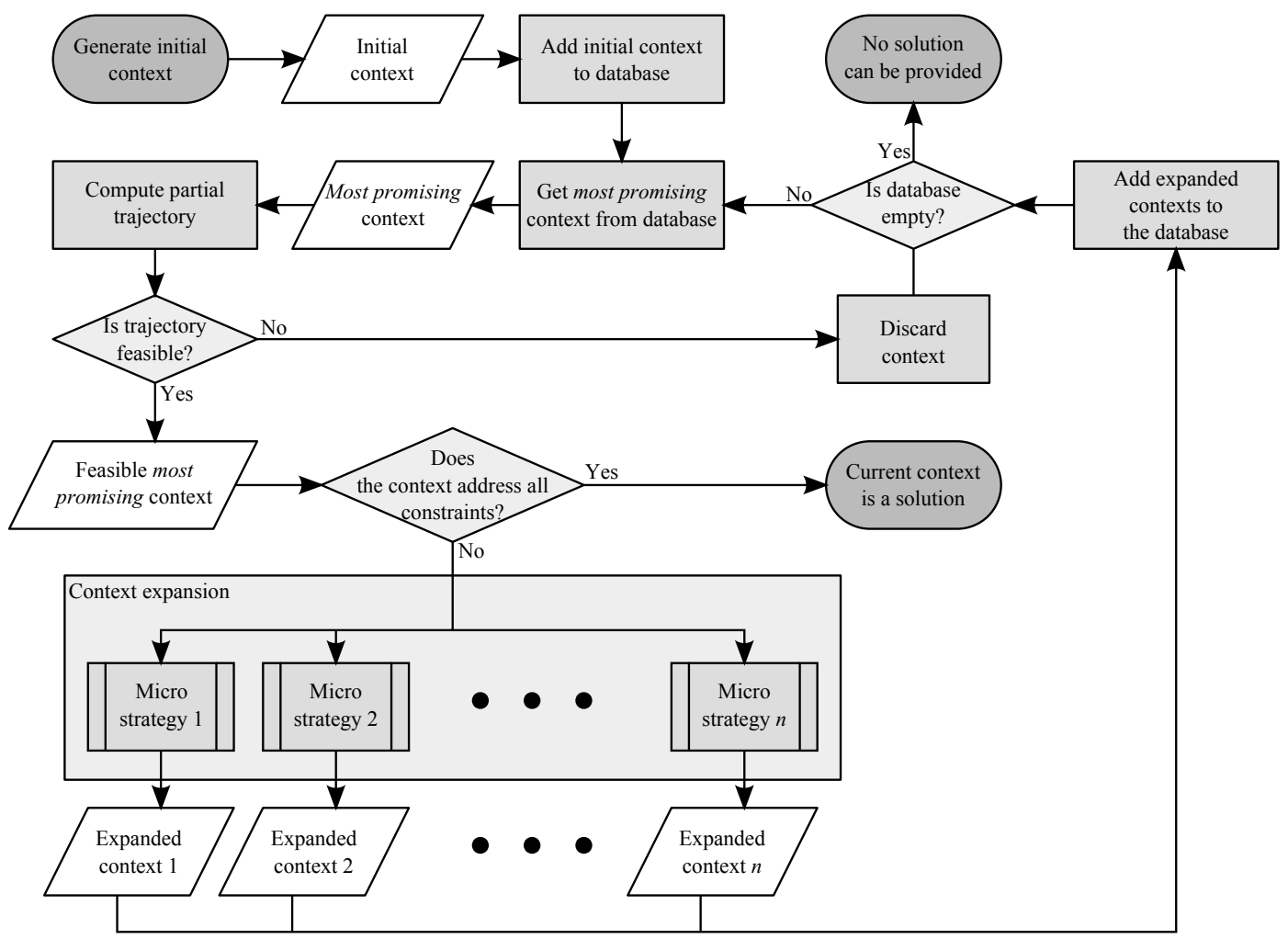

Figure 12.6: Vertical profile building flowchart. 
text, the composite and partial trajectory are empty (i.e., they contain no information), and all constraints are marked as unresolved (as they have not been addressed yet). This context has a score of zero.

2. The initial context is added to the context collection.

3. The most promising context - that is, the one obtaining the best score (i.e., the highest merit value or the lowest cost value) - is gathered and removed from the context collection.

4. The partial trajectory for the most promising context is obtained. This is done by translating the composite of the context into an AIDL word which is then integrated using the TCE. A special case occurs when the most promising context contains an empty composite (e.g., in the initial context); in such cases an empty trajectory is obtained as a result for this process (no integration is required).

5. The process checks whether or not the partial trajectory obtained in the previous step is feasible. Two basic checks are performed at this time. If the trajectory is not flyable (the TCE reported problems such as incompatibilities with the performance of the aircraft, insufficient fuel or unsafe manoeuvre), then the trajectory is not feasible. If the trajectory does not meet all the resolved constraints, then it is also not feasible. An empty trajectory is always feasible. If the trajectory is feasible, then the process jumps to step 9 .

6. If the trajectory is unfeasible, then the context is discarded.

7. The process checks whether the context collection is empty. If it is not empty, the process returns to step 3 , when the next most promising context is gathered.

8. If the context collection is empty, then the process is not able to provide a valid solution to the set of constraints provided. The process fails with no result, as it was unable to find any feasible aircraft intent (and associated trajectory) fulfilling all constraints.

9. If the most promising context is feasible, then the process checks whether this context still has unresolved constraints. If it does, the process jumps to step 11.

10. If the context has no unresolved constraints, then it has already integrated a feasible trajectory that meets all the constraints in the flight (during steps 4 and 5). That means that the action sequence in the context is a valid solution, and the process finishes providing the composite in the context as output.

11. If the context has one or more unresolved constraints, then it is expanded. Expanding a node means addressing one or more unresolved constraints, marking them as resolved. Some manoeuvres (expressed as ICDL composites) may be combined with the pre-existing partial composite in order to meet the addressed constraints during their execution interval, which also results in changes of the partial trajectory after trajectory computation by 


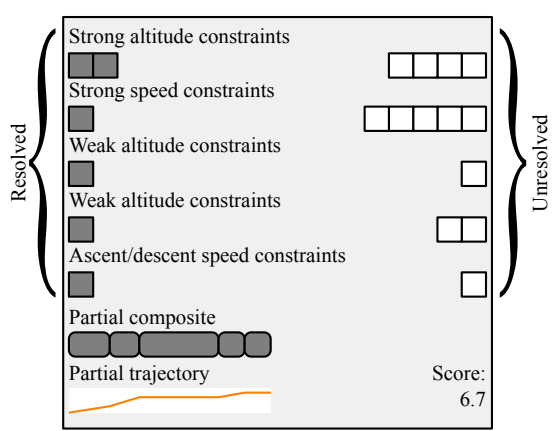

(a) Example original context.

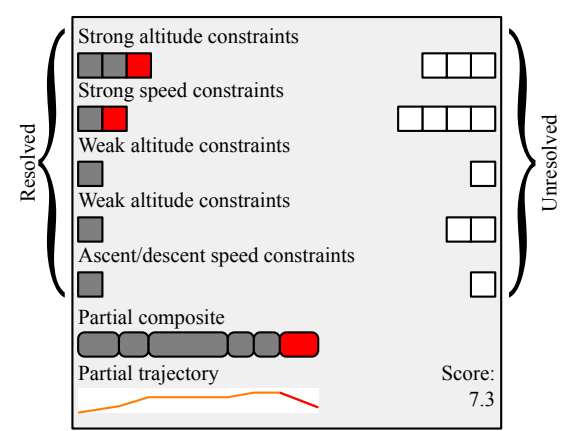

(b) Example expanded context.

Figure 12.7: Context expansion.

the TCE. An example context expansion is shown in figure 12.7, where the context in figure $12.7 \mathrm{a}$ is expanded to produce the context in figure $12.7 \mathrm{~b}$. The modular design of this process delegates the context expansion to several sub-modules called micro-strategies. Each micro-strategy has been designed to address a particular situation. For example, one micro-strategy focuses on altitude constraints requiring a constant speed ascent, while another micro-strategy focuses on combinations of altitude and speed constraints that require a Mach-CAS descent. If a micro-strategy cannot address any of the unresolved constraints, then it will return no result. A micro-strategy can return zero or more context expansions although, for simplicity, in figure 12.6 all micro-strategies return exactly one result.

12. All the expanded contexts generated in step 11 are added to the context collection. Then, the process continues in step 7 .

There are two key elements to the performance and optimality of this algorithm: the most promising criterion and the micro-strategies. These two elements can be seen as the configurable features of the process, and they are detailed in the following sections.

\subsubsection{Most promising criterion}

The most promising criterion can drastically impact the way the algorithm works. A context can be evaluated using a function $f(X)$, where $X$ is the evaluated context. The output of that function is the score of the context, and the context with the best score - the maximum, for merit functions, or minimum, for cost functions - is taken as the most promising.

The score can be either based on measurable values of the context or the trajectory -i.e., an actual value function $g(X)$ - , on estimations of values that cannot be measured - i.e., a heuristic value function $h(X)$-, or both. For example, if the cost of the flight in terms of fuel consumption was used as the most promising criterion, an actual value function $g(X)$ could provide the fuel consumption in the partial trajectory; whereas an heuristic value function $h(X)$ could provide an estimation of the fuel required to arrive to the destination. 
The most promising criterion may be related to the fuel consumption, traversed distance, flight time, user comfort; or even other magnitudes unrelated to the operating costs of the fight, such as the number of resolved or unresolved constraints.

Some well-known search strategies can be used in this process by picking the adequate merit/cost function. For example, if a merit function is defined as the number of resolved constraints, the process would behave as a depth-first search strategy [152]. Contexts with more resolved constraints, which are deeper into the tree, would have the highest merit values and therefore would be the first ones to be expanded further, until a leaf node is reached. This method aggressively tries to reach a leaf node as soon as possible. In the opposite case, where a merit function is defined as the number of constraints in the set of unresolved constraints, the process would behave as a breadth-first search algorithm [152]. This second type of search is highly inefficient for the problem studied in this paper.

An optimal solution to the discretized problem can be obtained if the score is designed so that the process behaves as an $\mathrm{A}^{*}$ (A-star) algorithm. In this case a cost function $f(X)=g(X)+h(X)$ is used, where $g(X)$ is the cost of the partial trajectory, and $h(X)$ is an estimation of the cost of the rest of the flight (which must not overestimate the real value). The particular definition of functions $g$ and $h$ depends on what is to be optimized. While an optimal discrete solution is guaranteed, this implementation can perform worse than others in computation time.

Choosing the right criteria for computing scores is crucial for the efficiency and optimality of the process. A purely heuristic merit function was found to be a good trade-off between output quality and computation time, making it possible to generate a valid solution in a short period of time (although optimality is not guaranteed). This merit function attempts to make the algorithm behave like a pilot or FMS. For each manoeuvre $m$ addressing a constraint $c \in X$, the 'likeliness of a pilot/FMS performing the manoeuvre $m$ for constraint $c$ ', denoted $p(m \mid c)$ is computed. The value for $p(m \mid c)$ is a real number between 0 and 1 , which can be determined using a simple expert system. This expert system produces a value for $p(m \mid c)$ based on the state of the aircraft (mainly altitude and speed) before the manoeuvre, the expected state of the aircraft after the manoeuvre, in addition to the addressed constraint and the manoeuvre itself.

This heuristic has been designed for the purpose of predicting aircraft trajectories in ATM, as the system attempts to reproduce typical pilot operations. The quality of this heuristic should be measured by comparing the output trajectories of this algorithm and actual trajectories from a real operational environment.

The value of the merit function is then computed as the sum of the individual values for each of the manoeuvres in the context:

$$
f(X)=\sum_{m, c \in X} p(m \mid c)
$$

This function has the additional advantage of allowing the recursive computation of the merit, in parallel with the expansion of the decision tree. 


\subsubsection{Micro-strategies}

The information on how to address different situations is encapsulated in different micro-strategies, which are isolated from one another. This allows modifying how constraints are addressed without changing the core of the process. Thus, if the behaviour of the algorithm in one particular situation has to be changed, there is no risk that the changes will affect behaviour in other situations. Another benefit of this approach is that micro-strategies can be enabled and disabled at wish. For example, one would want to disable micro-strategies related to CAS-Mach ascents or Mach-CAS descents when using the IGCPE to predict trajectories of UAs that fly at low altitudes. Therefore, it can be said that micro-strategy approach to the vertical profile generation improves maintainability of the algorithm.

The choice and development of micro-strategies is one of the critical factors for the efficiency and optimality of the algorithm. Micro-strategies are the elements of the process that generate the composite. They also discretize the search space, as only the actions contemplated in a micro-strategy can be generated in the process. In general, this means that the more different micro-strategies are used, the more optimal the result will be. However, too many micro-strategies can make the search space incomputable in practice. A limited number of microstrategies have been designed and used in a number of test scenarios; and these are able to generate the desired action sequences in most cases.

Our prototype includes the following micro-strategies, named after the manoeuvre they use to address the constraints:

- Ascent: uses a constant speed ascent manoeuvre to address an altitude constraint (with strong DoA). It also takes into account ascent speed constraints.

- Descent: analogous to the ascent micro-strategy, but uses a constant speed descent manoeuvre.

- Acceleration: uses a level thrust acceleration manoeuvre to address a speed constraint (with strong DoA).

- Deceleration: analogous to the acceleration micro-strategy, but uses a level thrust deceleration manoeuvre.

- Do nothing: resolves an altitude or speed constraint without adding a manoeuvre explicitly addressing that constraint (e.g., the constraint is already met as a result of other manoeuvre).

- $C A S /$ Mach ascent: addresses an altitude constraint and a speed constraint (with strong DoA). The speed constraint must be specified in Mach. Ascent speed constraints are taken into account.

- Mach/CAS descent: addresses an altitude constraint and a speed constraint (with strong DoA). The speed constraint must be specified in CAS. Descent speed constraints are taken into account.

- Energy share factor: addresses one altitude and one speed constraint (with strong DoA). It uses an ESF ascent with a given thrust regime manoeuvre, followed by a constant speed ascent manoeuvre. 
- Descent deceleration with path angle: uses a constant speed descent manoeuvre, followed by a constant path angle deceleration, to address one altitude and one speed constraint (with strong DoAs). It also takes into account descent speed constraints.

Most of the micro-strategies above have a 'weak' equivalent that address the same kind of constraints, but for weak DoAs.

The micro-strategies enumerated above use a series of ICDL templates, all of which are described in appendix C. The number and type of the templates used by one micro-strategy may vary depending on the context. Additionally, the ICDL allows manoeuvres to contain magnitude intervals, providing some flexibility in the specification of the trajectory. An interval provides a default value for a magnitude, and two boundaries (minimum and maximum) for the allowed values. For example, an ascent manoeuvre could have a target altitude specified as an interval with a default value of $6400 \mathrm{ft}$, and allowed values [6400,7000]. Micro-strategies specify manoeuvres using intervals whenever possible, so that the particular values can be optimized at a later time. As explained before, in the developed prototype the COE performs this optimization of the trajectory.

\subsection{Results}

In this section, the flexibility of the proposed algorithm for the generation of the vertical profile is assessed. First, it will be shown how this algorithm can be used to reproduce the behaviour of the trajectory predictor in an FMS. The merit function and micro-strategies described in this chapter have been designed so that the output trajectory of the IGCPE is as close to a real trajectory -i.e., the trajectory flown by an FMS-equipped aircraft - as possible. Since most FMSs generate trajectories based on a pre-defined profile, the baseline vertical profile from the EFMS [85] (see the phase table of figure 3.4 in chapter 3) is used to compute the reference trajectory.

The scenario of a commercial flight from the Adolfo Suárez Madrid-Barajas Airport (ICAO: LEMD) to the Seville Airport (ICAO: LEZL) is considered. The aircraft used in this scenario is a Boeing 737-800. A flight intent has been generated containing all the intermediate waypoints of the flight, all the necessary constraints, and the optimization objectives. Using the notation in appendix D, this flight intent can be written as:

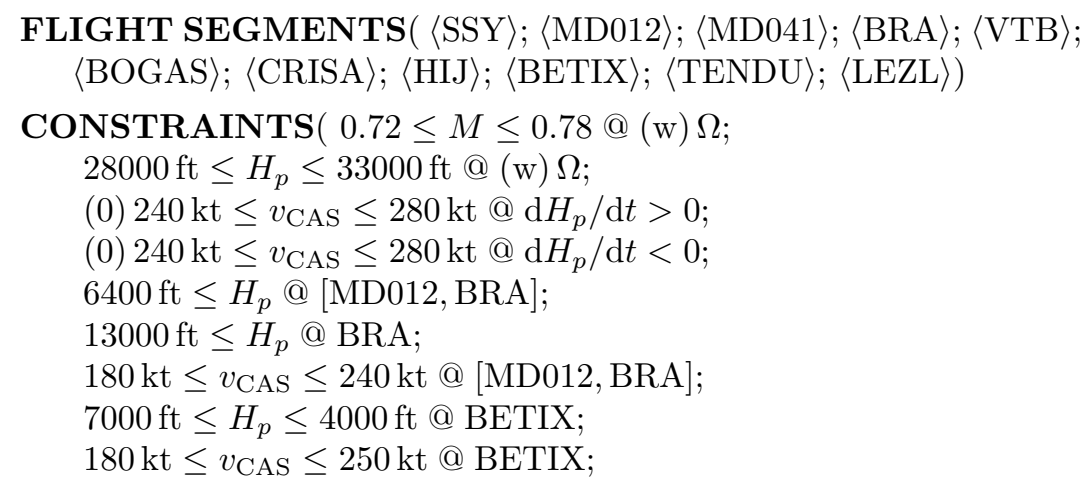


$H_{p}=2000 \mathrm{ft} @ \mathrm{LEZL} ;$

$\left.180 \mathrm{kt} \leq v_{\mathrm{CAS}} \leq 210 \mathrm{kt} @ \mathrm{LEZL}\right)$

OBJECTIVES $\left((1)-\mathrm{d} m_{f} / \mathrm{d} t @ \Omega ;(20) 1 @ \Omega\right)$

This flight intent has been translated into a sampled trajectory using two different methods. The first one (method $A$ ) uses the IGCPE and the microstrategy decision tree algorithm described in this chapter to generate the vertical profile. The composite resulting from this translation can be seen in figure 12.9 (the micro-strategy that generated each composite is shown above the composite). The second one (method $B$ ) uses a modified IGCPE, in which the algorithm for generating the vertical profile has been replaced by a pre-defined vertical profile (based on [85]). Constraints are employed to complete the missing magnitudes in this pre-defined profile. The trajectory resulting from using the method $A$ will be referred to as the generated trajectory, and the one resulting from using method $B$, reference trajectory. Figure 12.8 shows both trajectories.

The generated and reference trajectories may seem identical at first sight, but there are subtle differences. These differences can be spotted when looking at the altitudes (figure 12.10) and speeds (figure 12.11) of both flights. Microstrategies could be modified to make the result of method $A$ even more similar to the reference trajectory. However, the generated trajectory may be sufficiently

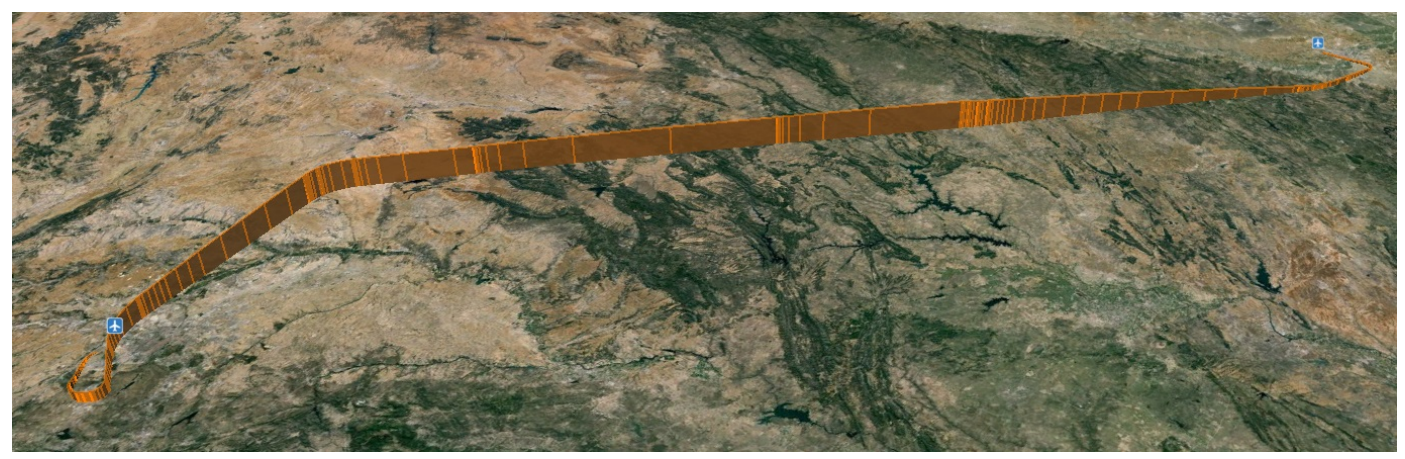

(a) Trajectory produced using a micro-strategy decision tree (generated trajectory).

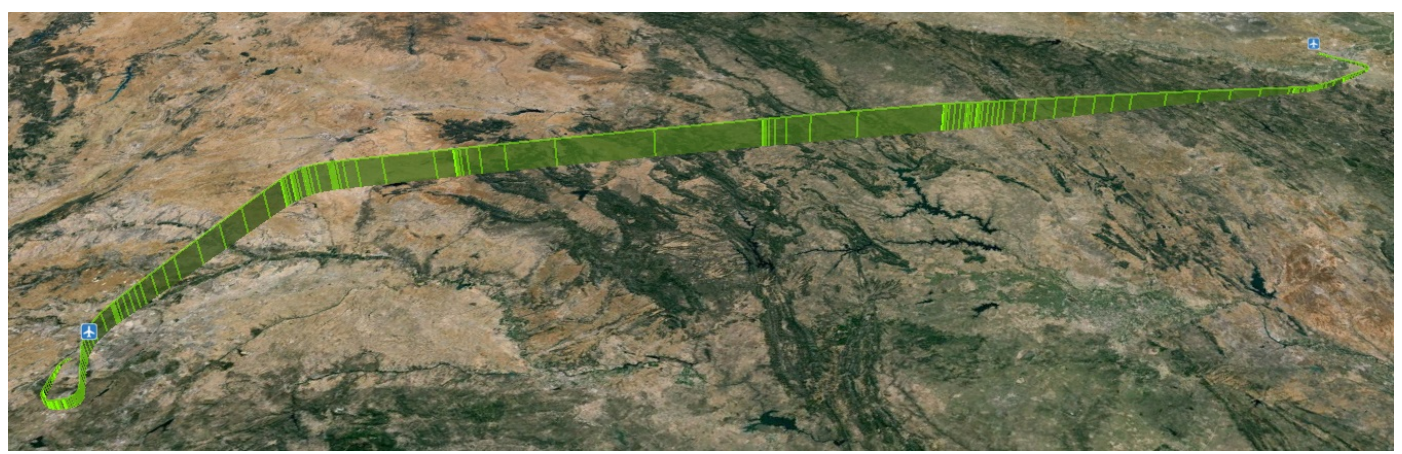

(b) Trajectory produced using a pre-defined profile (reference trajectory).

Figure 12.8: Trajectories produced by the IGCPE (Map data: Google, Landsat). 


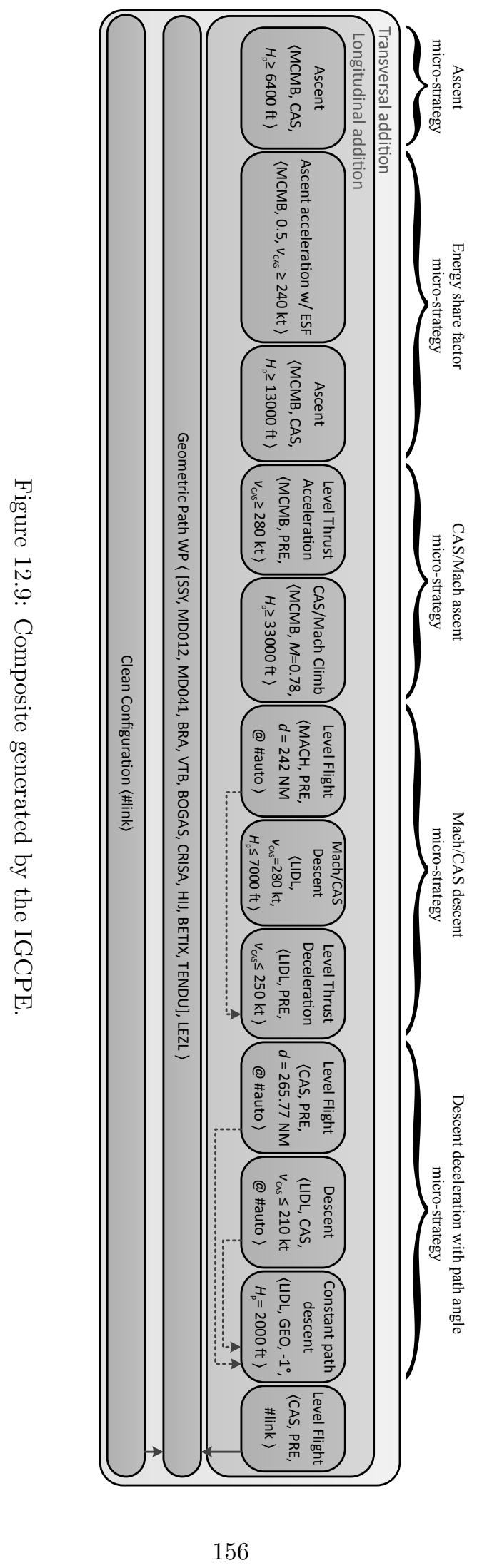




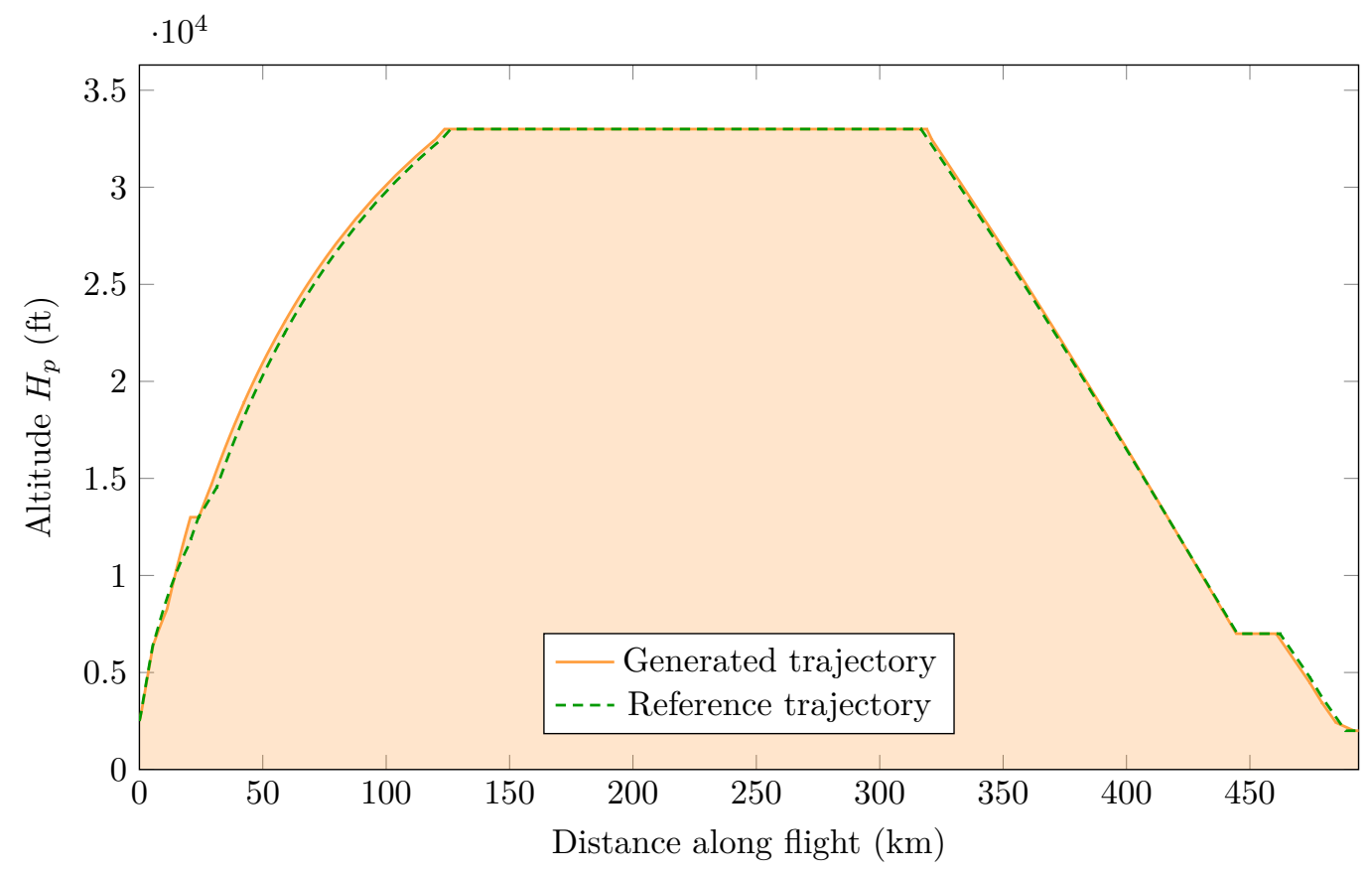

Figure 12.10: Barometric altitude of flights along distance.

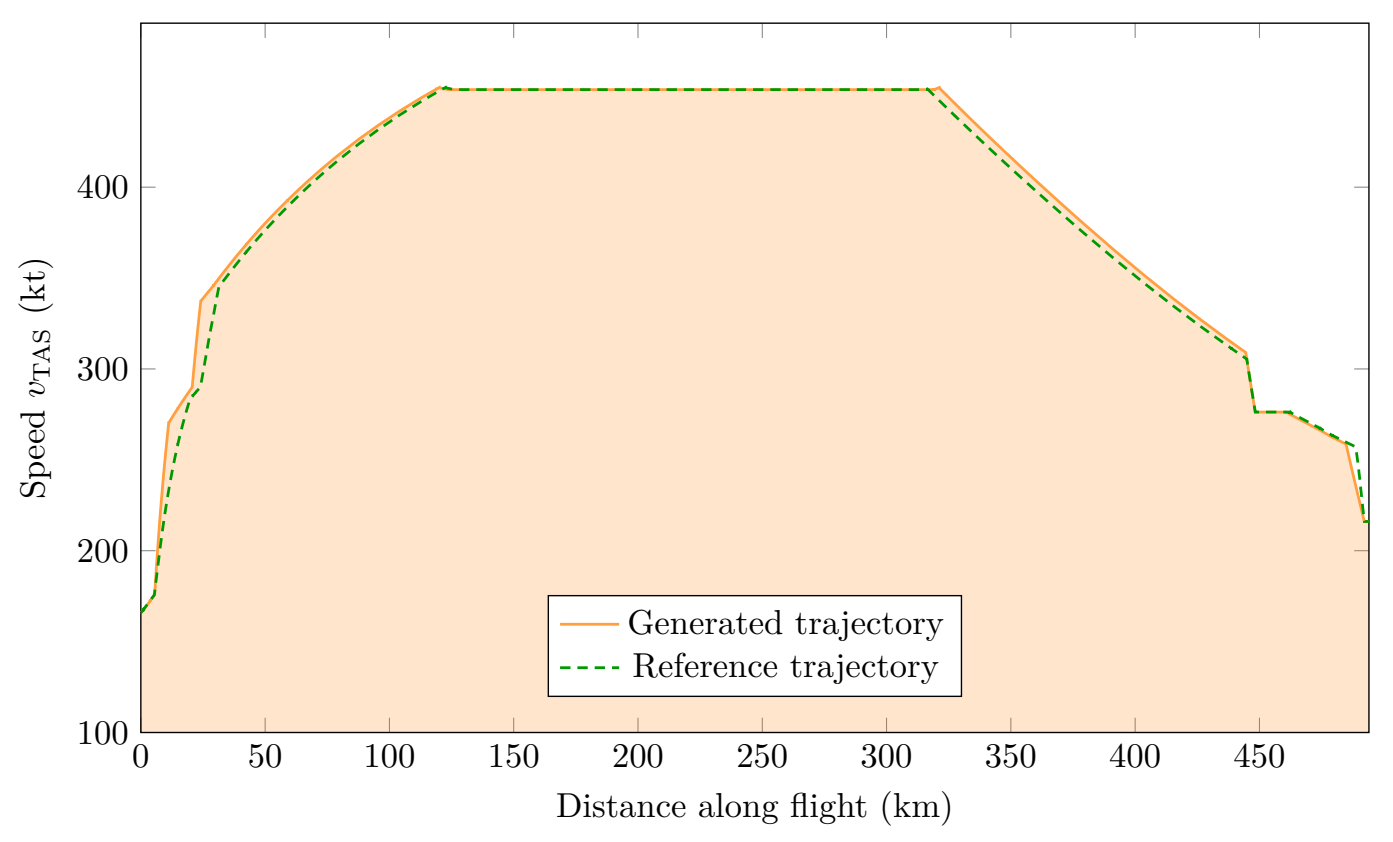

Figure 12.11: TAS of flights along flight distance. 


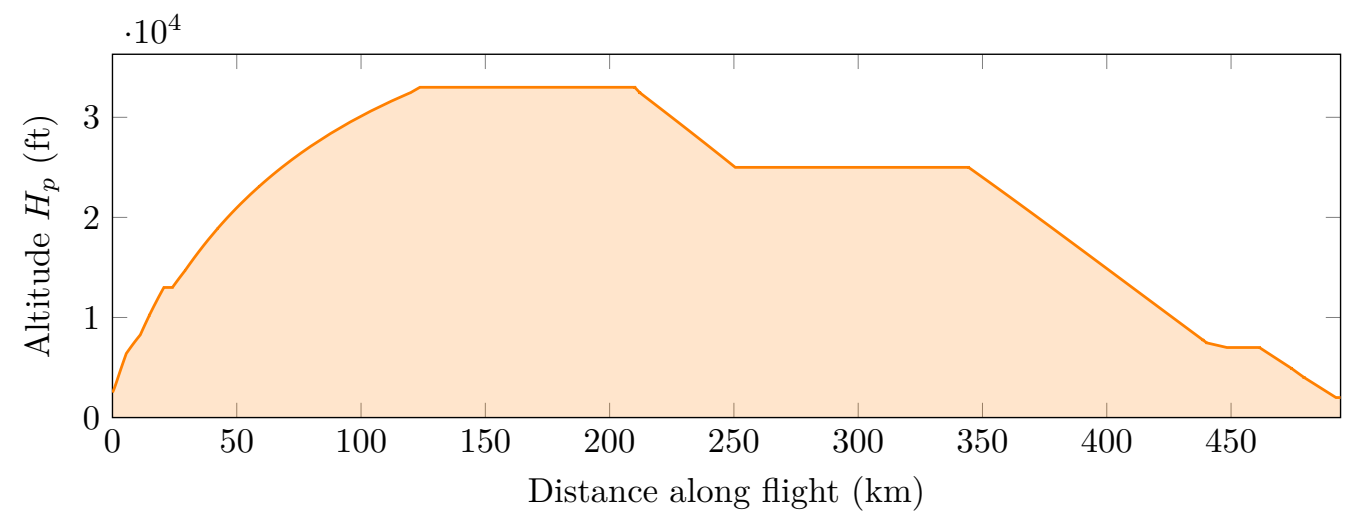

(a) Barometric altitude of trajectory along distance.

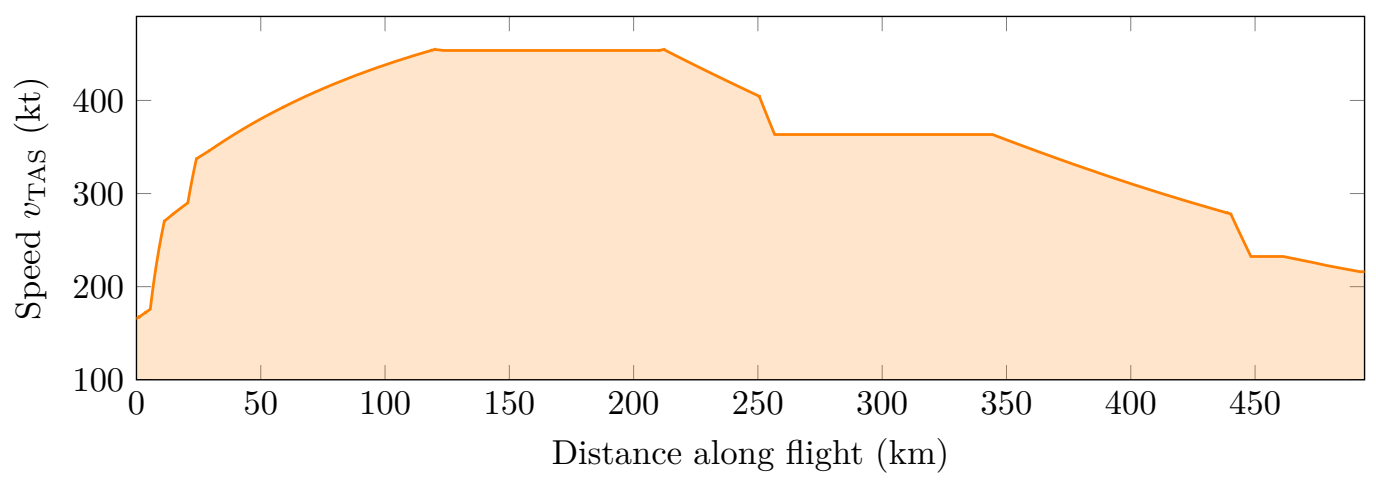

(b) TAS of trajectory along distance.

Figure 12.12: Altitude and speed of generated flight (with extra constraint).

accurate for the requirements of a ground-based trajectory predictor. Moreover, the micro-strategy decision tree algorithm (the one used in method $A$ ) can easily deal with additional constraints in the flight intent: since it is not based on a predefined profile, this algorithm can deal with more constraints along the flight, at the expense of more computational cost. An additional constraint is included in the example shown in figure 12.12. This example requires the aircraft to descend to 25,000 feet very early during cruise. In the notation in appendix D, this constraint is written:

$$
H_{p} \leq 25000 \mathrm{ft} @ \text { CRISA }
$$

This descent doesn't match a typical descent profile, but to the microstrategy decision tree algorithm it is as difficult to solve as any other constraint. The composite corresponding to this example is included in figure 12.13.

Some results obtained after generating a trajectory using both methods are shown in table 12.1. Note that, for method $A$, two results are provided: they correspond to the initial scenario and the one with the additional constraint. The results include the computational cost (the time it takes the trajectory predictor to generate the output trajectory), and the operating cost of the flight. 


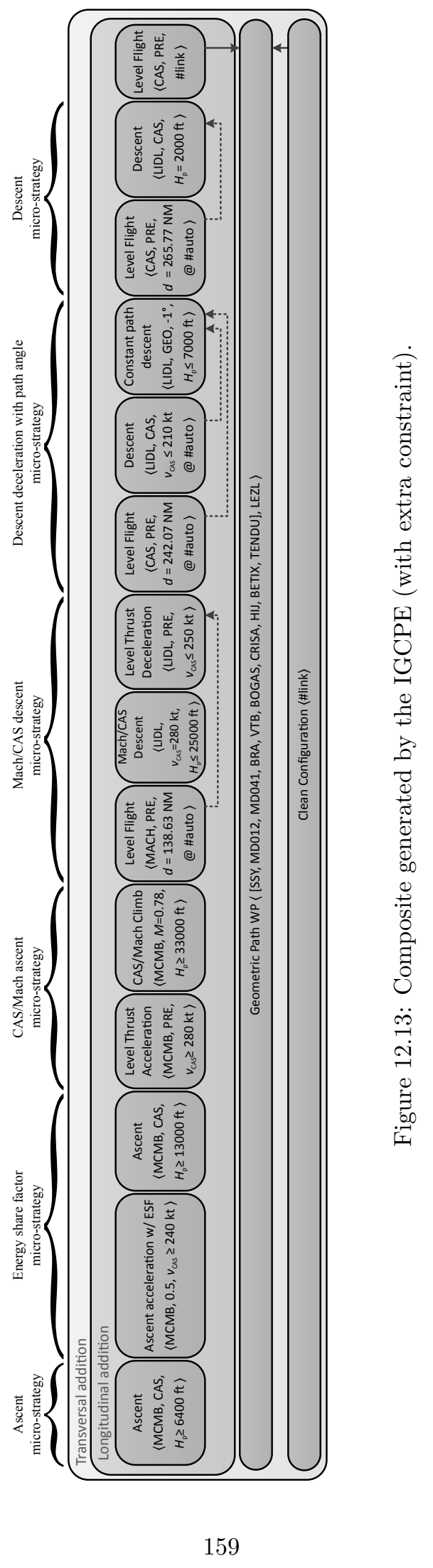




\begin{tabular}{lcrrrr}
\hline \multirow{2}{*}{ Trajectory } & \multirow{2}{*}{$\begin{array}{c}\text { Optimi- } \\
\text { zation }\end{array}$} & \multicolumn{2}{c}{$\begin{array}{c}\text { Computation } \\
\text { time (seconds) }\end{array}$} & \multicolumn{2}{c}{$\begin{array}{c}\text { Cost } \\
\text { (pounds) }\end{array}$} \\
\cline { 3 - 6 } & & \multicolumn{1}{c}{ Mean } & \multicolumn{1}{c}{$\sigma$} & \multicolumn{1}{c}{ Mean } & \multicolumn{1}{c}{$\sigma$} \\
\hline \hline \multirow{2}{*}{ Generated } & No & 4.271 & 0.061 & 3908.41 & 0 \\
& Yes & 208.651 & 48.350 & 3776.457 & 24.267 \\
\hline Generated & No & 4.194 & 0.056 & 3918.57 & 0 \\
(extra const.) & Yes & 214.601 & 127.652 & 3855.445 & 37.320 \\
\hline \multirow{2}{*}{ Reference } & No & 1.914 & 0.013 & 4051.2 & 0 \\
& Yes & 223.012 & 55.645 & 3812.106 & 25.760 \\
\hline
\end{tabular}

Table 12.1: Trajectory computation times and costs

The operating cost is computed using the objectives of the flight intent, which is equivalent to computing the cost using a CI of 20 (see section 8.1.4). The IGCPE can perform an optimization of the trajectory provided by the algorithm generating the vertical profile (either using method $A$ or $B$ ). The optimization algorithm is non-deterministic, and therefore the results may vary considerably in each simulation. The values shown in table 12.1 are the mean and standard deviation obtained after 20 simulations. Results are provided with optimization enabled and disabled.

Some examples of the parameters resulting from the optimization of the composite's intervals are shown in table 12.2. The parameters correspond to those magnitudes in figure 12.9, although in that figure the intervals were omitted (due to limited space).

\begin{tabular}{rrrr}
\hline Default value & \multicolumn{1}{c}{ Interval } & $\begin{array}{c}\text { Optimized } \\
\text { value } 1\end{array}$ & \multicolumn{1}{c}{$\begin{array}{c}\text { Optimized } \\
\text { value } 2\end{array}$} \\
\hline \hline $6400 \mathrm{ft}$ & {$[6400,7000]$} & $6400.06 \mathrm{ft}$ & $6998.23 \mathrm{ft}$ \\
$240 \mathrm{kt}$ & {$[180,240]$} & $236.76 \mathrm{kt}$ & $238.76 \mathrm{kt}$ \\
$13000 \mathrm{ft}$ & {$[13000,33000]$} & $20470.4 \mathrm{ft}$ & $14988.75 \mathrm{ft}$ \\
$280 \mathrm{kt}$ & {$[240,280]$} & $262.01 \mathrm{kt}$ & $268.89 \mathrm{kt}$ \\
0.78 & {$[0.72,0.78]$} & 0.72 & 0.72 \\
$33000 \mathrm{ft}$ & {$[28000,33000]$} & $32951.44 \mathrm{ft}$ & $32788.58 \mathrm{ft}$ \\
$280 \mathrm{kt}$ & {$[240,280]$} & $254.12 \mathrm{kt}$ & $255.01 \mathrm{kt}$ \\
$7000 \mathrm{ft}$ & {$[4000,7000]$} & $6783.92 \mathrm{ft}$ & $6829.56 \mathrm{ft}$ \\
$250 \mathrm{kt}$ & {$[180,250]$} & $246.88 \mathrm{kt}$ & $220.95 \mathrm{kt}$ \\
$210 \mathrm{kt}$ & {$[180,210]$} & $186.66 \mathrm{kt}$ & $208.79 \mathrm{kt}$ \\
\hline \hline & Cost (pounds) & 3749.75 & 3777.45 \\
\hline
\end{tabular}

Table 12.2: Optimization result examples

These results show that the algorithm proposed in this paper takes more time to compute a trajectory, but the differences are negligible compared to the time required by the optimization process, which requires a significant amount of time. Also, with optimization enabled, each execution provides very different results, whereas the algorithms without optimization are very consistent in their output and the time it takes to compute a particular trajectory.

Adding more constraints usually requires more computation time, but in this scenario that is not the case. These examples show that the computation 
time required isn't easily predictable when using the algorithm presented in this paper, as it depends on multiple factors such as the number and type of constraints, the micro-strategies, and the most promising criterion; and these factors interact in ways that cannot always be anticipated.

\subsection{Final words}

This chapter has detailed the design and implementation of the IGCPE in the trajectory predictor developed in collaboration with BR\&TE. The algorithm used to generate the horizontal profile is similar to existing methods, but a novel algorithm for the generation of the vertical profile is presented. This new algorithm is different from existing ones in that it is not based on predefined profiles and later amendment of the profile based on constraints, nor on the intersection of forwards and backwards integration processes. Instead, this algorithm evaluates each constraint. A number of micro-strategies evaluate the possible manoeuvres that may address the constraint, and the most promising candidate is chosen. If, later on, the chosen micro-strategy can't provide a feasible trajectory, the algorithm can go back and choose a different candidate.

These two key elements, the micro-strategies and the most promising criterion, are still under development and being improved to increase the overall performance of the IGE. Nevertheless, with the current implementation, results show that the vertical profile algorithm is flexible enough to mimic the behaviour of other trajectory predictors. The additional flexibility allows using this trajectory predictor to be used in many different scenarios, changing the micro-strategies if necessary. For example, the algorithm doesn't assume the flight to have any fixed flight phases, so it can be used in non-commercial flights where these phases may not be relevant. The main caveat of the algorithm is the increased computational cost, which may be very difficult to predict in some cases.

Chapters 11 and 12 have provided an understanding of the structure and algorithms of the trajectory predictor presented in this thesis. However, the applications of TLPEs and the language hierarchy described in part II are not limited to ATM. The remaining chapters will show some applications of TLPEs to UAS. In particular, the next chapter develops a TCE for the computation of trajectory descriptions expressed in QR-AIDL. 



\section{Chapter 13}

\section{Computation of quadrotor trajectories}

The TLPEs described in previous chapters were initially conceived for their use in an ATM context. However, many of these engines can be adapted for their use in other contexts, such as UAS. One example of such engines is the trajectory computation engine (TCE), which translates aircraft intents into sampled trajectories. The TCE was originally designed to compute trajectories of fixed-wing aircraft. Therefore, the FW-AIDL variant of the AIDL is used to express the aircraft intent provided to that engine. A general description of the TCE was included in chapter 11, and additional details can be found in [78] and [16].

This chapter describes an analogous TCE that computes trajectories of quadrotor aircraft. From a user's perspective, the main difference between the TCE described in previous chapters and this TCE is the use of the QR-AIDL variant of the AIDL to express the input aircraft intent. Other than that, this TCE has the same input and output as the the TCE designed for an ATM context, as shown in figure 13.1.

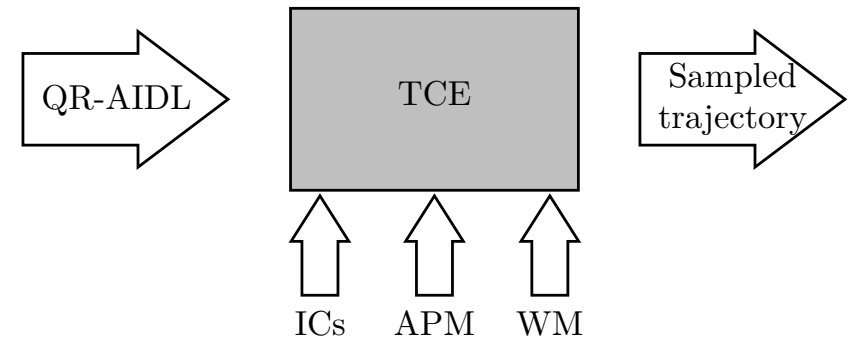

Figure 13.1: Trajectory computation engine for quadrotor aircraft.

An overview of the computation process carried out inside the TCE is provided in section 13.1. However, this chapter focuses on describing the results obtained using a prototyped TCE. The integration of several possible combinations of QR-AIDL instructions, denoted operations, is analysed in section 13.2. 
Finally, a complete aircraft intent expressed in QR-AIDL is computed, and the results of this computation are shown in section 13.3.

\subsection{Engine description}

The methodology used to integrate an AIDL trajectory was already explained in chapter 6 . This methodology is called sequential DAEs resolution (SDR), and consists on solving a DAE for each possible combination of AIDL instructions along the flight. Each of these combinations is denoted an operation.

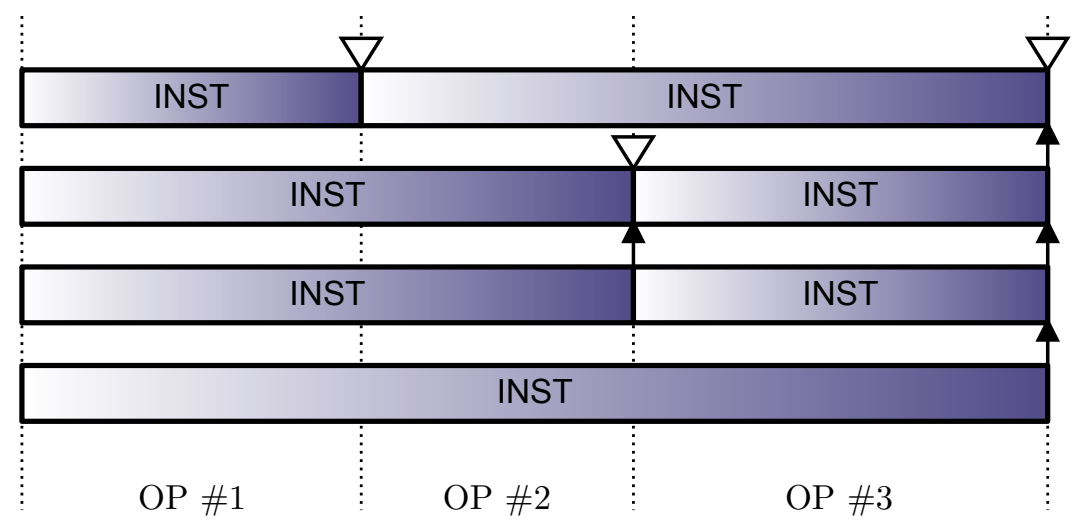

Figure 13.2: Operations in QR-AIDL word.

Figure 13.2 shows an example QR-AIDL word with three operations. Following the SDR methodology, the TCE must keep track of the instructions conforming each operation. The first operation comprises the first instruction in each thread. Each of these instructions represents an algebraic constraint. These algebraic constraints, with the equations of motion of the aircraft, form a DAEs describing the movement of the aircraft during the operation. This DAE can be solved for the consistent initial conditions provided to the TCE, and generating an integrated trajectory for that operation. The integration of the operation must stop when any end trigger of any of the instructions becomes active. In the next operation, the instructions that have ended (that is, whose end trigger has been reached) are replaced by the next instruction in the same thread. Operations are integrated sequentially until the end of the AIDL word is reached. Resulting from this process, a trajectory corresponding with the whole AIDL word is obtained.

The methodology described above is identical between the TCEs designed for ATM and UAS. The main differences between both TCEs are in the equations of motion and the instruction - and their associated constraints - used in the integration of each operation. A prototype TCE for the integration of quadrotor trajectories has been developed. It uses numerical integration methods for the computation of the trajectories. In particular, Newton/direct methods are used to solve the DAE system. This prototype, however, doesn't support all the features available in the QR-AIDL, so it can only compute trajectories described 
using a subset of the QR-AIDL. This subset includes all the QR-AIDL instructions described in chapter 6, but not all the parameters (e.g., it is not possible to define arbitrary functions as parameters). Additionally, auto triggers are not supported in this prototype.

The integration of several different operations using this prototype is showcased in the next section.

\subsection{Operation integration}

In chapter 6, the QR-AIDL was derived. In that derivation, six combinations of constraints were considered indispensable for the description of quadrotor trajectories. These combinations were summarized in table 6.4. This section provides an example operation for each of the combinations, detailing the input and output of the integration process.

The weather model (WM) and aircraft performance model (APM) used are the same for all the integrated trajectories in this chapter. The WM includes the wind speed vector (in this case, wind speed is zero in all axes) and the air density (a value of $1.25 \mathrm{~kg} / \mathrm{m}^{3}$ is used). The APM contains the aerodynamic parameters of the quadrotor, which are detailed in section B.3.

\subsubsection{Combination 1}

The first combination of constraints defines a flight in terms of the horizontal speed (provided using polar coordinates), vertical speed, and yaw.

There are several combinations of QR-AIDL instructions that translate into such constraints. In the example provided here, the horizontal speed is set using a horizontal speed law and a course law - the former defines the module of the speed and the latter defines the direction. Two additional law instructions define the vertical speed and the yaw in the operation. The operation stops at time $t=20 \mathrm{~s}$, as defined by a fixed trigger. This combination of instructions is illustrated in figure 13.3.

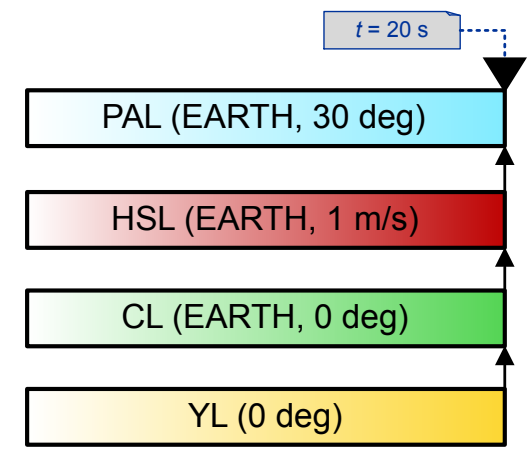

Figure 13.3: Operation using the first combination of instructions.

The QR-AIDL instructions in the operation, with the corresponding parameters, can be translated into a set of constraints using the equations detailed 
in section 6.3.1. Each instruction in figure 13.3 corresponds to one of the constraints below (in the same order):

$$
\begin{aligned}
& \dot{z}_{e}-v_{e} \sin 30^{\circ}=0 \\
& \begin{cases}\dot{x}_{e}-1 \mathrm{~m} / \mathrm{s} \cos \chi_{e}=0 & \text { if }\left|\cos \chi_{e}\right|>\left|\sin \chi_{e}\right| \\
\dot{y}_{e}-1 \mathrm{~m} / \mathrm{s} \sin \chi_{e}=0 & \text { otherwise }\end{cases} \\
& \dot{y}_{e} \cos 0^{\circ}-\dot{x}_{e} \sin 0^{\circ}=0 \\
& \psi-0^{\circ}=0
\end{aligned}
$$

According to table 6.4, this combination of constraints has 3 initial DoFs That is, the initial values of three variables must be provided by the user. In particular, the three variables that must be present in the initial conditions are $x_{e}, y_{e}$, and $z_{e}$. Additionally, the initial time must be provided, $t_{0}=0$. The rest of the variables can be computed to obtain some consistent initial conditions. The initial position of the aircraft is defined to be:

$$
\boldsymbol{\zeta}=\left[\begin{array}{l}
x_{e} \\
y_{e} \\
z_{e}
\end{array}\right]=\left[\begin{array}{l}
0 \\
0 \\
0
\end{array}\right]
$$

The operation can then be integrated by solving the DAE formed by the equations of motion of the quadrotor (see section 6.2.2) and the constraints above. The values for all the variables in the system during the execution interval of the operation (in this case, between 0 and 20 seconds) are obtained. Figure 13.4 shows the evolution of the variables $x_{e}, y_{e}$, and $z_{e}$ over time.

Aircraft's position

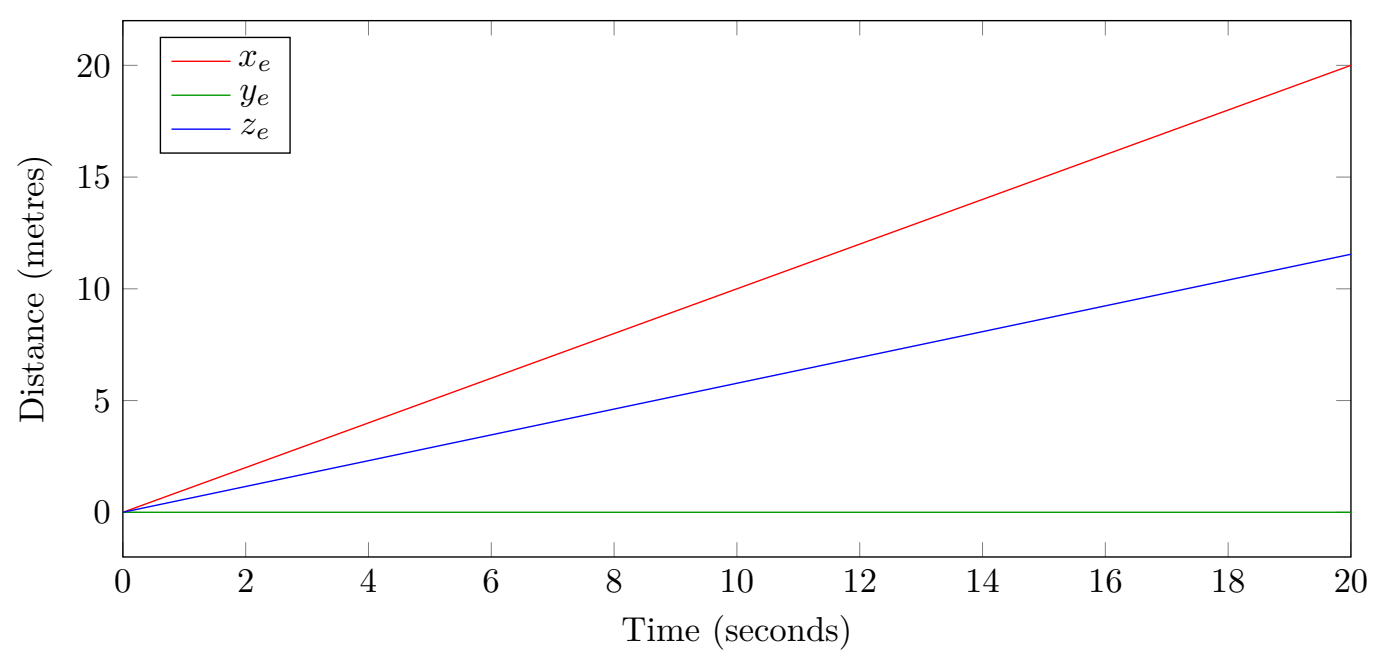

Figure 13.4: Evolution of the aircraft's coordinates with time.

In this case, as the horizontal and vertical speeds are constant, the evolution of the aircraft's position is linear. The orientation of the quadrotor (roll, pitch, 
and yaw) is constant, and the propellers rotate at a constant angular speed of $356.123 \mathrm{rad} / \mathrm{s}$ to maintain the speed and path angle.

\subsubsection{Combination 2}

A flight can also be defined in terms of the horizontal speed (in polar coordinates), thrust, and yaw. Unlike the previous combination, a constraint affecting the thrust is used, replacing the vertical speed constraint.

An example set of QR-AIDL instructions that produce this combination of constraints is presented here. A horizontal speed law and a hold course instruction are used to define the horizontal speed in polar coordinates. A yaw law instruction defines the yaw, and a thrust law instruction defines the thrust. In this case, a condition on the aircraft's state is used to define the end trigger $\left(z_{e}=100 \mathrm{~m}\right)$. The resulting QR-AIDL word is shown in figure 13.5.

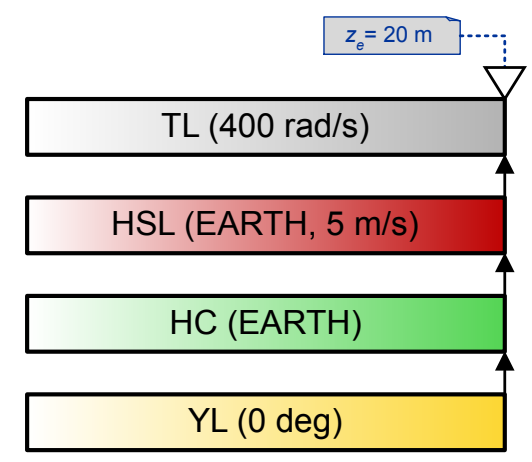

Figure 13.5: Operation using the second combination of instructions.

The instructions in this QR-AIDL word, including their respective parameters, correspond to the following constraints:

$$
\begin{aligned}
& \Omega-400 \mathrm{rad} / \mathrm{s}=0 \\
& \begin{cases}\dot{x}_{e}-5 \mathrm{~m} / \mathrm{s} \cos \chi_{e}=0 & \text { if }\left|\cos \chi_{e}\right|>\left|\sin \chi_{e}\right| \\
\dot{y}_{e}-5 \mathrm{~m} / \mathrm{s} \sin \chi_{e}=0 & \text { otherwise }\end{cases} \\
& \dot{y}_{e} \cos \chi_{e, 0}-\dot{x}_{e} \sin \chi_{e, 0}=0 \\
& \psi-0^{\circ}=0
\end{aligned}
$$

This combination of constraints has 4 initial DoFs, that is, four variables of the aircraft must be provided to generate some consistent initial conditions. These four variables correspond to the initial position of the aircraft $\boldsymbol{\zeta}$ and the initial vertical speed $\dot{z}_{e}$. Additionally, the hold course instruction requires the initial course $\chi_{e, 0}$ to be defined. In this example, the initial conditions provided 
to the TCE are:

$$
\begin{aligned}
\boldsymbol{\zeta}=\left[\begin{array}{l}
x_{e} \\
y_{e} \\
z_{e}
\end{array}\right] & =\left[\begin{array}{l}
0 \\
0 \\
0
\end{array}\right] \\
\dot{z}_{e} & =0 \\
\chi_{e, 0} & =0
\end{aligned}
$$

The time of these initial conditions, $t_{0}=0$, must also be provided so the execution interval is completely defined. With all the information above, the TCE can compute a sampled trajectory.

The evolution of the aircraft's position during this operation is shown in figure 13.6a. As the horizontal speed is constant during the operation, the evolution of the $x_{e}$ and $y_{e}$ coordinates is linear. The vertical speed increases from 0 to $11.29 \mathrm{~m} / \mathrm{s}$ during the operation, as shown in figure $13.6 \mathrm{~b}$. At first, the acceleration $\ddot{z}_{e}$ is higher, but as the quadrotor moves faster, the friction reduces this acceleration and the speed stabilizes (see figure 13.6c).

\section{Aircraft's position}

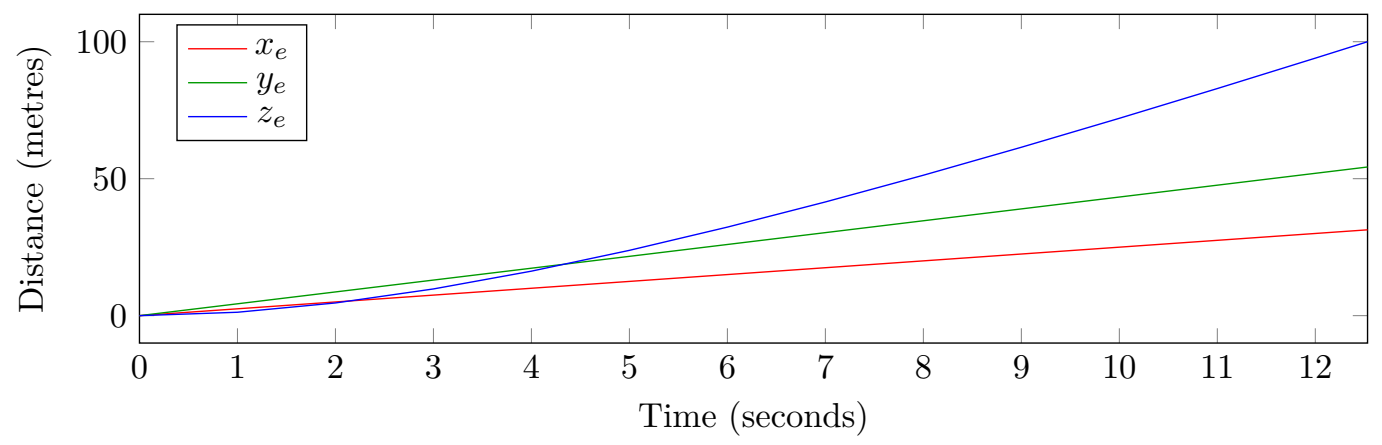

(a)

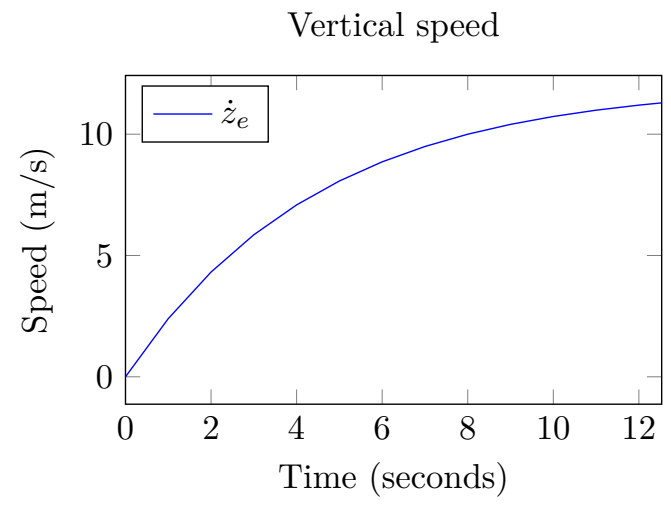

(b)

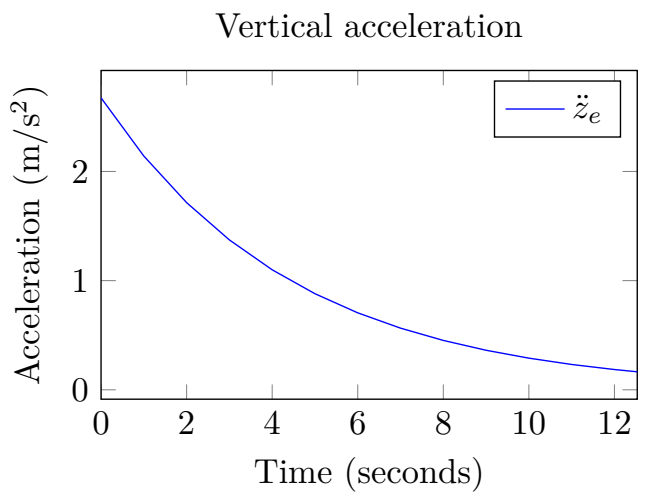

(c)

Figure 13.6: Evolution of several variables of the aircraft's state vector with time (combination two). 


\subsubsection{Combination 3}

A different combination of constraints defines the flight in terms of the horizontal speed (in polar coordinates), altitude, and yaw.

One example QR-AIDL word that produces such combination of constraints is presented here. A hold altitude instruction is used to maintain the altitude constant. As in previous cases, a horizontal speed law and a course law instruction are used to define the horizontal speed using polar coordinates. In this case, a function parameter is provided to the horizontal speed law instruction, making the speed increase with a constant acceleration of $0.5 \mathrm{~m} / \mathrm{s}^{2}$. Finally, a yaw law instruction is used to set the yaw angle. The end trigger in this QR-AIDL word is a floating trigger that makes the operation stop when the horizontal speed (i.e., ground speed) $v_{g}$ reaches $10 \mathrm{~m} / \mathrm{s}^{2}$. This QR-AIDL word is shown in figure 13.7 .

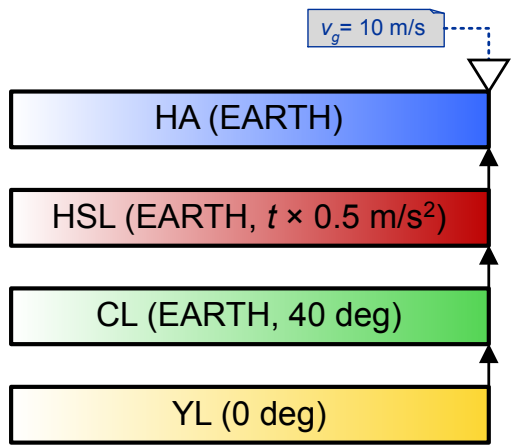

Figure 13.7: Operation using the third combination of instructions.

The instructions in figure 13.7, with their parameters, can be translated into the constraints below:

$$
\begin{aligned}
& z_{e}-z_{e, 0}=0 \\
& \begin{cases}\dot{x}_{e}-t \cdot 0.5 \mathrm{~m} / \mathrm{s}^{2} \cos \chi_{e}=0 & \text { if }\left|\cos \chi_{e}\right|>\left|\sin \chi_{e}\right| \\
\dot{y}_{e}-t \cdot 0.5 \mathrm{~m} / \mathrm{s}^{2} \sin \chi_{e}=0 & \text { otherwise }\end{cases} \\
& \dot{y}_{e} \cos 40^{\circ}-\dot{x}_{e} \sin 40^{\circ}=0 \\
& \psi-0^{\circ}=0
\end{aligned}
$$

The number of initial DoFs of this set of constraints, according to table 6.4, is 2. This means that the values of two variables in the state vector must be provided. These two variables are $x_{e}$ and $y_{e}$. Additionally, the hold altitude instruction requires the initial altitude, $z_{e, 0}$ to be provided. So, in order to obtain some consistent initial conditions, the aircraft's initial position must be defined:

$$
\boldsymbol{\zeta}=\left[\begin{array}{l}
x_{e} \\
y_{e} \\
z_{e}
\end{array}\right]=\left[\begin{array}{l}
0 \\
0 \\
0
\end{array}\right]
$$

With the equations of motion of the quadrotor, the constraints, and some 
consistent initial conditions, the operation can be integrated. The evolution of some of the variables in the state vector are shown in figure 13.8.

Aircraft's position

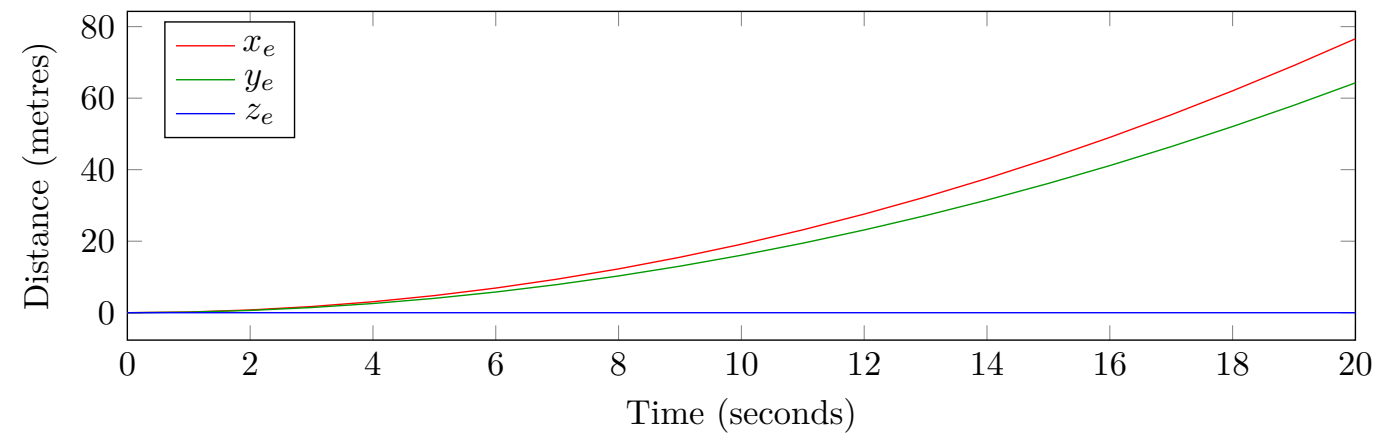

(a)

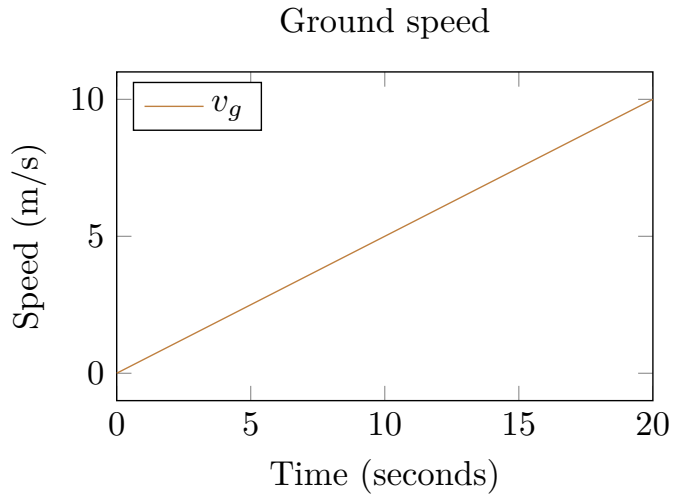

(b)

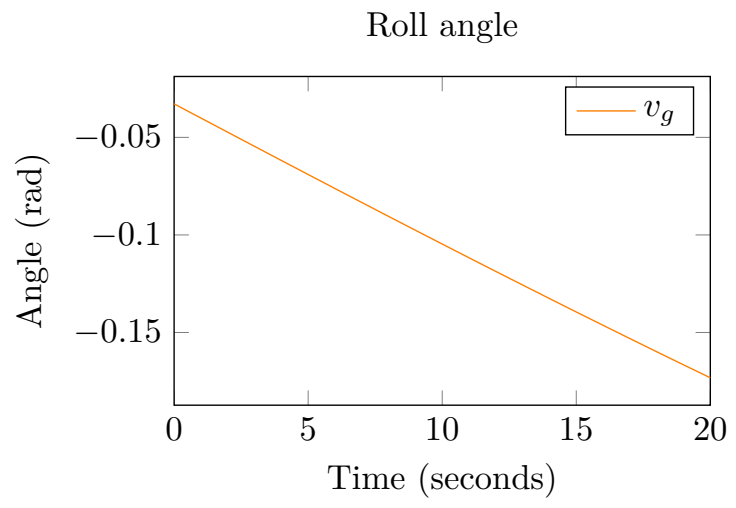

(d)
Propeller speed

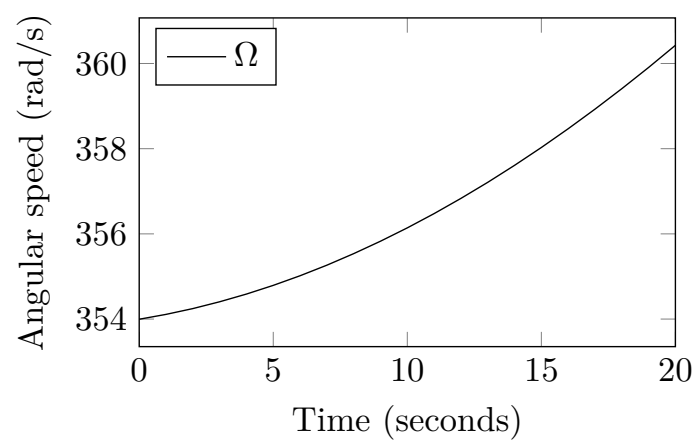

(c)

Pitch angle

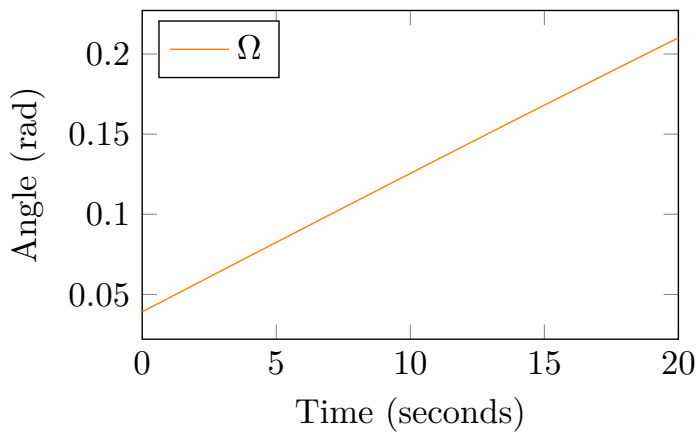

(e)

Figure 13.8: Evolution of several variables of the aircraft's state vector with time (combination three). 
The evolution of the aircraft's position can be seen in figure 13.8a. The aircraft moves in the horizontal plane with increasing velocity, whereas the altitude is held constant. The evolution of the aircraft's horizontal speed (groundspeed) is shown in figure $13.8 \mathrm{~b}$. The horizontal speed increases linearly with time, as defined by the horizontal speed law instruction. As the speed increases, so does the friction, requiring more thrust to maintain the acceleration constant. Therefore, the propeller speed must increase to compensate this friction, as illustrated in figure 13.8c. Figures $13.8 \mathrm{~d}$ and $13.8 \mathrm{e}$ show how the orientation of the aircraft also changes to counteract the increasing friction.

\subsubsection{Combination 4}

An alternative means to define a quadrotor's flight is by defining the vertical speed, roll, pitch and yaw.

In the example QR-AIDL word presented here, law instructions are used to define each of those aspects of the flight. A vertical speed law defines a constant vertical speed of $1 \mathrm{~m} / \mathrm{s}$. A roll law, pitch law, and yaw law define the orientation of the aircraft. The end of this operation is set using a fixed trigger with the condition $t=30 \mathrm{~s}$. This QR-AIDL word is shown in figure 13.9.

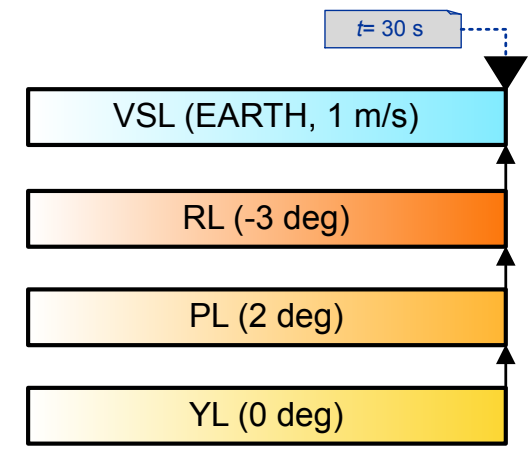

Figure 13.9: Operation using the fourth combination of instructions.

Those QR-AIDL instructions represent the following algebraic constraints:

$$
\begin{aligned}
& \dot{z}_{e}-1 \mathrm{~m} / \mathrm{s}=0 \\
& \phi+3^{\circ}=0 \\
& \theta-2^{\circ}=0 \\
& \psi-0^{\circ}=0
\end{aligned}
$$

This combination of constraints has 5 initial DoF. This means that 5 variables from the aircraft's state vector must be defined to obtain some consistent initial conditions. These variables are the aircraft's position $\boldsymbol{\zeta}$ and the horizontal speed (defined in either cartesian or polar coordinates). In this case, the 
initial conditions are:

$$
\begin{aligned}
\boldsymbol{\zeta}=\left[\begin{array}{l}
x_{e} \\
y_{e} \\
z_{e}
\end{array}\right] & =\left[\begin{array}{l}
0 \\
0 \\
0
\end{array}\right] \\
\dot{x}_{e} & =0 \\
\dot{y}_{e} & =0
\end{aligned}
$$

Once all the required information has been defined, the operation can be computed.

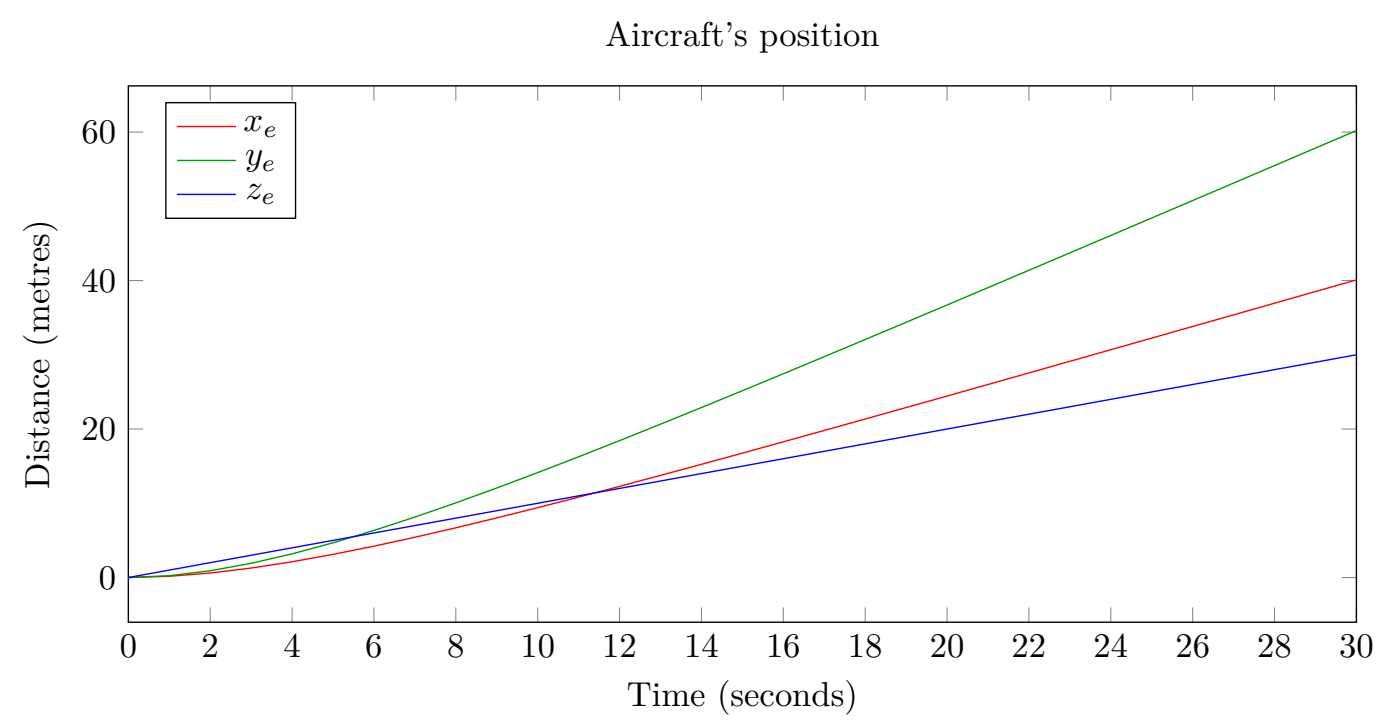

(a)

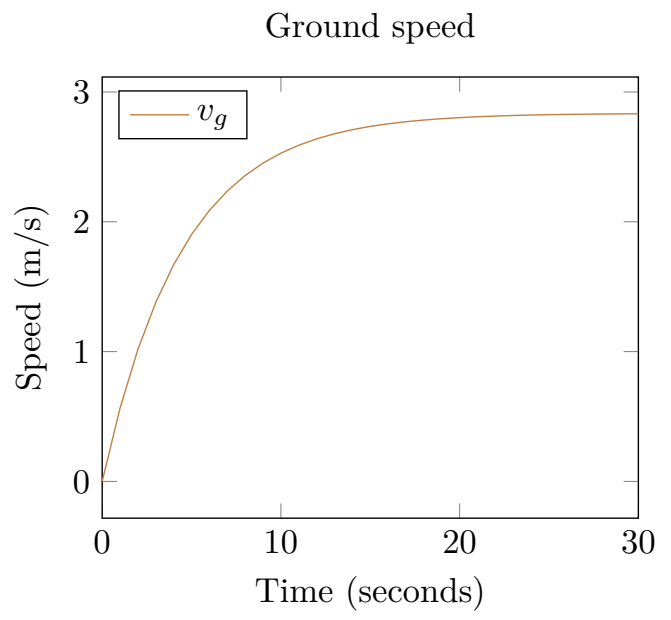

(b)

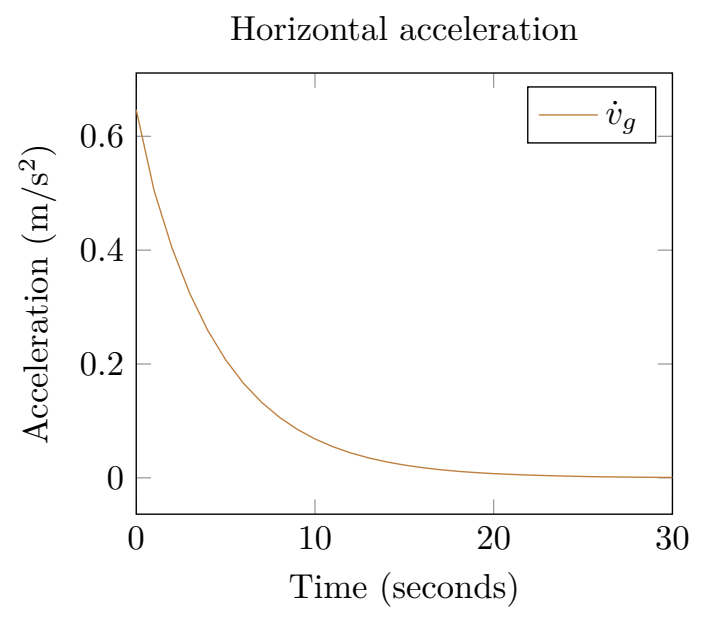

(c)

Figure 13.10: Evolution of several variables of the aircraft's state vector with time (combination four). 
The evolution of the aircraft's position with time is shown in figure 13.10a. Given the specified vertical speed and the orientation of the quadrotor, a constant propeller speed of $358.116 \mathrm{rad} / \mathrm{s}$ must be maintained. This thrust and the orientation produce a horizontal acceleration. The change in the aircraft's speed is illustrated in figure $13.10 \mathrm{~b}$, and the corresponding acceleration is shown in figure $13.10 \mathrm{c}$.

\subsubsection{Combination 5}

In the QR-AIDL, it is possible to define a flight using the four control variables in the system ${ }^{1}$. This results in a flight defined by the thrust, roll, pitch and yaw.

There are several instructions that define each of the control variables mentioned above. In the example presented here, law instructions are used to set the thrust, roll, and pitch; and a hold yaw instruction is used to set the yaw. In this case, the propeller speed set by the thrust law instruction is inferior to the speed necessary to maintain altitude, and the quadrotor will descend. The operation stops when the altitude of $z_{e}=0$ is reached, as defined by the floating trigger in figure 13.11.

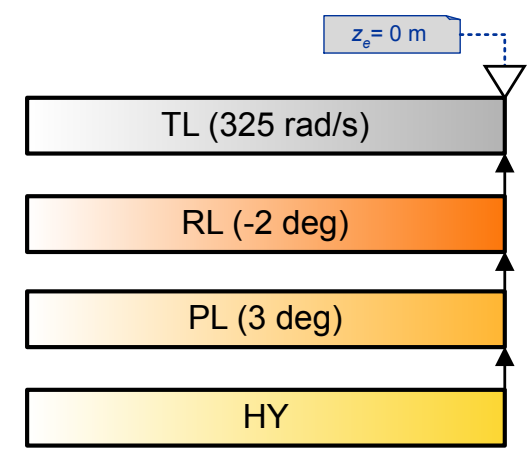

Figure 13.11: Operation using the fifth combination of instructions.

The instructions in figure 13.11 can be translated into four algebraic equations, which are included below:

$$
\begin{aligned}
& \Omega-325 \mathrm{rad} / \mathrm{s}=0 \\
& \phi+2^{\circ}=0 \\
& \theta-3^{\circ}=0 \\
& \psi-\psi_{0}=0
\end{aligned}
$$

The combination of constraints above has six initial DoFs. Six state variables, comprising the position $\boldsymbol{\zeta}$ and speed $\mathbf{v}$ of the aircraft along the three axes, must be explicitly defined in the initial conditions. Additionally, the hold yaw

\footnotetext{
${ }^{1}$ Unlike the FW-AIDL [16], where one of the control variables is the lift, which cannot be directly controlled.
} 
instruction requires the initial yaw to be specified. In this example, the initial conditions include the following information:

$$
\begin{gathered}
\boldsymbol{\zeta}=\left[\begin{array}{c}
x_{e} \\
y_{e} \\
z_{e}
\end{array}\right]=\left[\begin{array}{c}
0 \\
0 \\
50
\end{array}\right] \\
\mathbf{v}=\left[\begin{array}{c}
\dot{x}_{e} \\
\dot{y}_{e} \\
\dot{z}_{e}
\end{array}\right]=\left[\begin{array}{l}
0 \\
0 \\
5
\end{array}\right] \\
\psi=0
\end{gathered}
$$

A sampled trajectory can be computed by the TCE with this information. In this trajectory, the aircraft initially continues to ascend, as the initial vertical speed is positive. But, as the thrust is not sufficient to maintain the altitude, the vertical speed decreases until the aircraft starts descending. The thrust and the orientation of the quadrotor also produce a lateral acceleration.

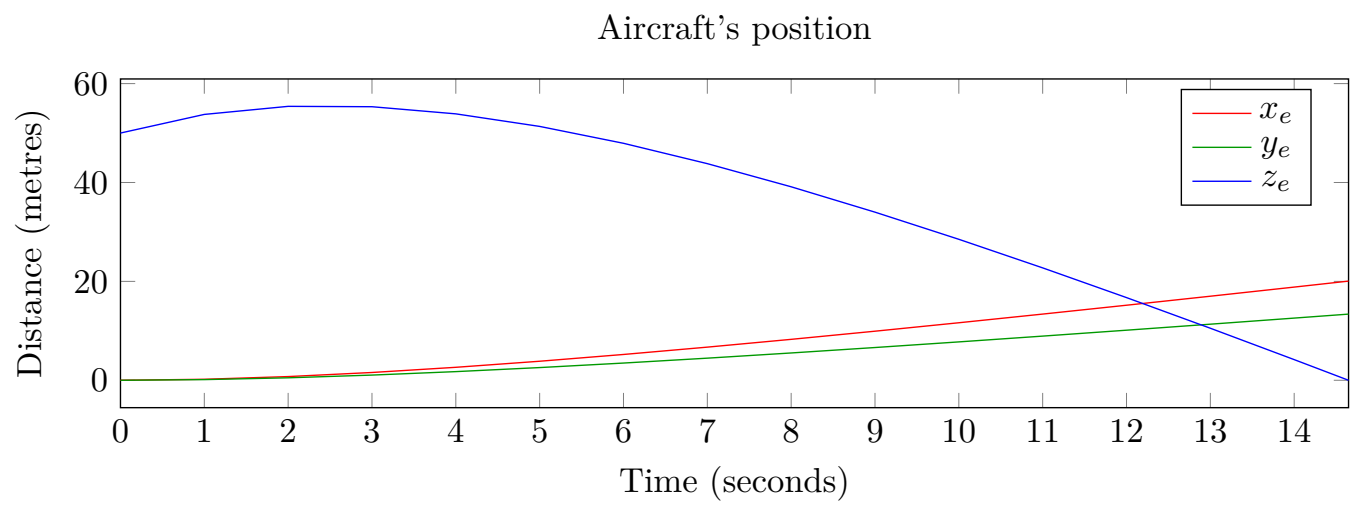

(a)

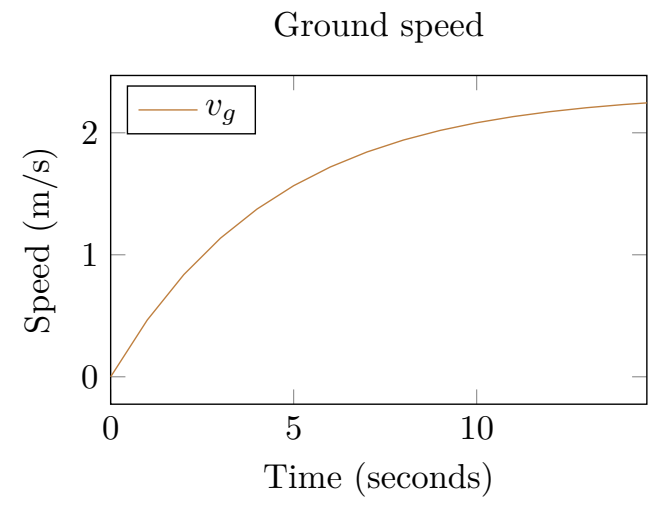

(b)

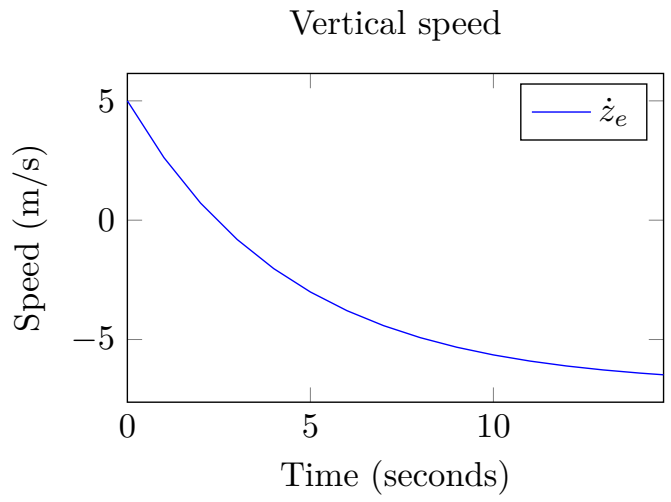

(c)

Figure 13.12: Evolution of several variables of the aircraft's state vector with time (combination five). 
The movement of the aircraft during this operation can be observed in figure 13.12a. The evolution of the horizontal and vertical speeds are shown in figures $13.12 \mathrm{~b}$ and $13.12 \mathrm{c}$, respectively. The operation stops at $t=14.6485$, when the condition in the floating trigger $\left(z_{e}=0 \mathrm{~m}\right)$ is met.

\subsubsection{Combination 6}

The last combination of constraints included in table 6.4 defines a flight in terms of the altitude, roll, pitch and yaw.

An example QR-AIDL word that translates into this combination of constraints is included here. A hold altitude instruction is used to define the altitude, and law instructions are used to set the orientation (roll, pitch, and yaw) of the quadrotor. In this example, the direction of the horizontal speed depends on the yaw angle, which varies at a rate of 6 degrees per second. The operation should end after 30 seconds, as defined by a fixed trigger. The example QR-AIDL word used in this section is shown in figure 13.13.

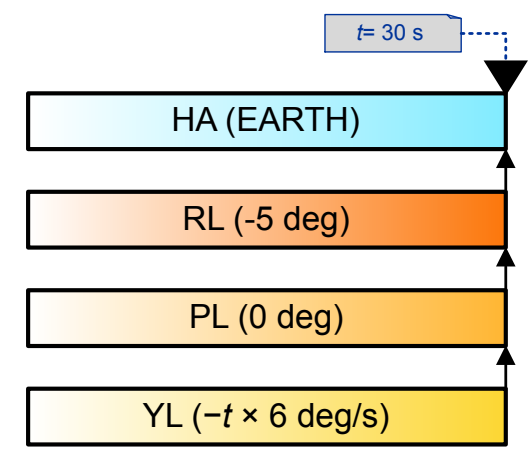

Figure 13.13: Operation using the sixth combination of instructions.

The four instructions in that QR-AIDL word represent the same number of algebraic constraints. The equivalence between QR-AIDL instructions and algebraic constraints is detailed in section 6.3.1. In this case, the algebraic constraints corresponding to the AIDL instructions in the operation are:

$$
\begin{aligned}
& z_{e}-z_{e, 0}=0 \\
& \phi+5^{\circ}=0 \\
& \theta-0^{\circ}=0 \\
& \psi+t \cdot 6^{\circ} / \mathrm{s}=0
\end{aligned}
$$

This combination of constraints has 4 initial DoFs, so four state variables must be provided to the TCE. These four variables are the horizontal position (defined by variables $x_{e}$ and $y_{e}$ ) and the horizontal speed (defined in polar or cartesian coordinates). Additionally, the hold altitude instruction requires the initial altitude to be specified. In this case, the initial conditions provided to 
the TCE are:

$$
\begin{gathered}
\boldsymbol{\zeta}=\left[\begin{array}{l}
x_{e} \\
y_{e} \\
z_{e}
\end{array}\right]=\left[\begin{array}{l}
0 \\
0 \\
0
\end{array}\right] \\
\dot{x}_{e}=0 \\
\dot{y}_{e}=0
\end{gathered}
$$

Given the equations of motion of the quadrotor, the algebraic constraints, and some consistent initial conditions, a trajectory for the operation can be computed. Figure 13.14a shows the evolution of the aircraft's position. The altitude is constant during the whole operation, as defined by the hold altitude instruction. To maintain the altitude constant, the propeller speed must remain at $\Omega=354.44 \mathrm{rad} / \mathrm{s}$. This propeller speed and the orientation of the aircraft produce a horizontal acceleration. Since the aircraft rotates around its $y_{b}$ axis (i.e., the yaw changes), the direction of this acceleration also changes with time.

Aircraft's position

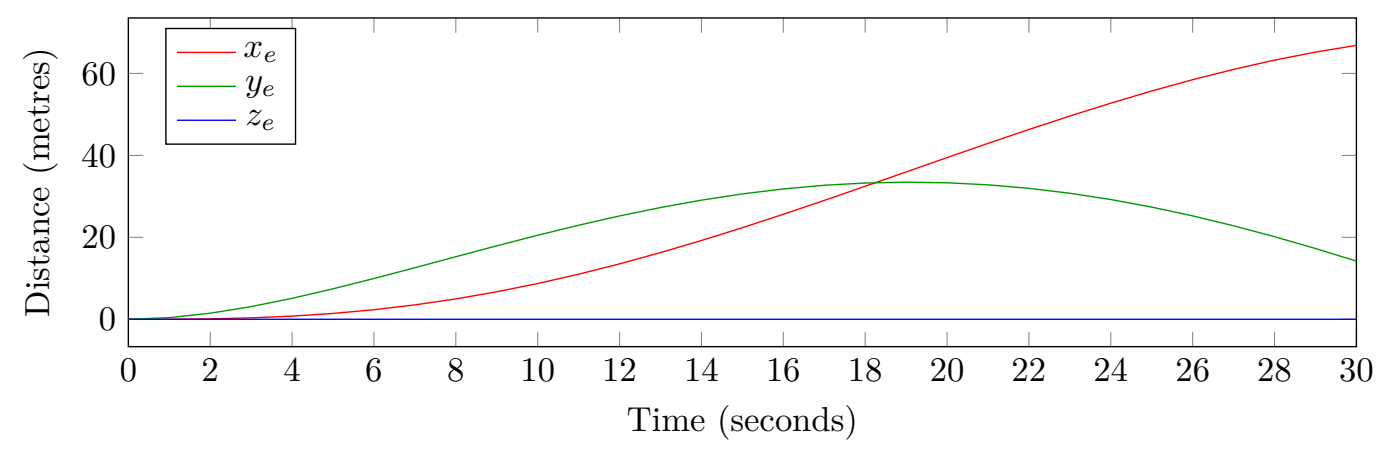

(a)

Horizontal accelerations

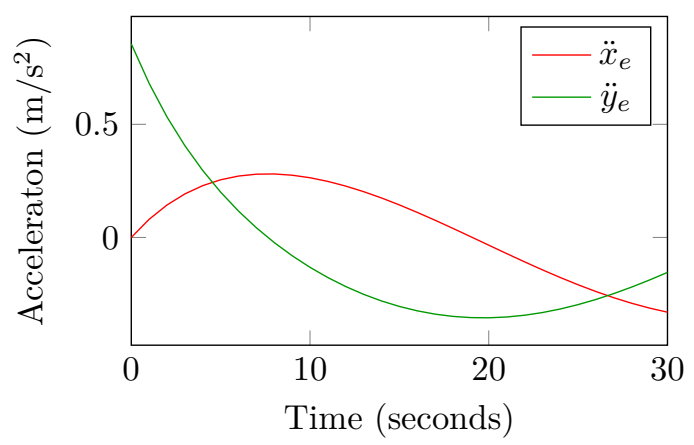

(b)

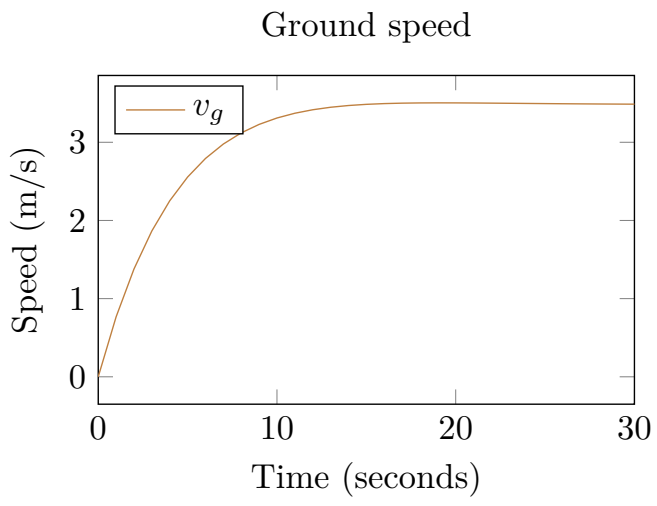

(c)

Figure 13.14: Evolution of several variables of the aircraft's state vector with time (combination six). 
The evolution of this acceleration (given in cartesian coordinates $\ddot{x}_{e}$ and $\ddot{y}_{e}$ ) is depicted in figure 13.14b. The module of the speed is shown in figure 13.14c. It increases from zero to approximately $3.5 \mathrm{~m} / \mathrm{s}$.

\subsection{Example}

The examples in the previous section show the trajectories resulting from the integration of one operation in the TCE. This section provides a complete example in which the aircraft intent contains several operations that must be computed using the SDR method. This example uses the same WM and APM used in the previous examples.

In this example, geographic coordinates (i.e., longitude and latitude) are used, as the example intends to describe a real scenario in an urban environment. A conversion between the geographic coordinates and the corresponding cartesian coordinates are provided in table 13.1. The origin of coordinates of the cartesian reference frame is set at the take-off location, with the $x_{e}$ axis pointing to the East and the $y_{e}$ axis pointing to the North. The complete aircraft intent, described using the graphical notation, is provided in figure 13.15. This aircraft intent describes a flight around the Escuela Técnica Superior de Ingenieros de Telecomunicación (ETSIT), which could be used to explore part of the perimeter of one of the buildings. The quadrotor is expected to take-off at one of the parking lots, and finally land on the basketball court. Changes in speed are governed by a horizontal speed law, which uses a constant acceleration of $0.5 \mathrm{~m} / \mathrm{s}^{2}$. The acceleration is described using a linear function where $t_{0}$ represents the time at which the execution of the instruction started.

\begin{tabular}{c|c|c|c|c}
\multirow{2}{*}{ Point } & \multicolumn{2}{|c}{ Cartesian } & \multicolumn{2}{c}{ Geographic } \\
& $x_{e}$ & $y_{e}$ & Longitude & Latitude \\
\hline \hline$\# 1$ & $0 \mathrm{~m}$ & $0 \mathrm{~m}$ & $3.7270^{\circ} \mathrm{W}$ & $40.4530^{\circ} \mathrm{N}$ \\
$\# 2$ & $-10.76 \mathrm{~m}$ & $73.92 \mathrm{~m}$ & $3.7271^{\circ} \mathrm{W}$ & $40.4537^{\circ} \mathrm{N}$ \\
$\# 3$ & $173.31 \mathrm{~m}$ & $102.97 \mathrm{~m}$ & $3.7249^{\circ} \mathrm{W}$ & $40.4539^{\circ} \mathrm{N}$ \\
$\# 4$ & $125.54 \mathrm{~m}$ & $-82.60 \mathrm{~m}$ & $3.7255^{\circ} \mathrm{W}$ & $40.4523^{\circ} \mathrm{N}$
\end{tabular}

Table 13.1: Waypoints of flight at ETSIT

The sampled trajectory corresponding to this aircraft intent is shown in figure 13.16. It can be seen how the aircraft intent is designed to ensure that the aircraft avoids any obstacles such as buildings or trees. More information on the resulting trajectory is provided in figures $13.17 \mathrm{a}$ and $13.17 \mathrm{~b}$, which show the altitude and speed profiles, respectively. 


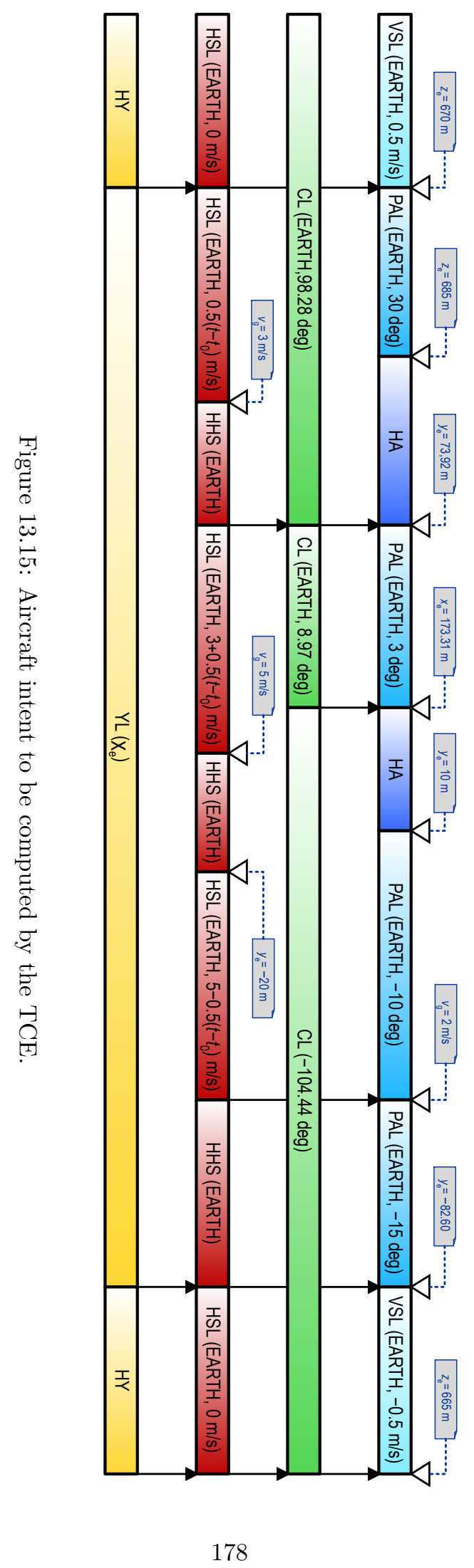




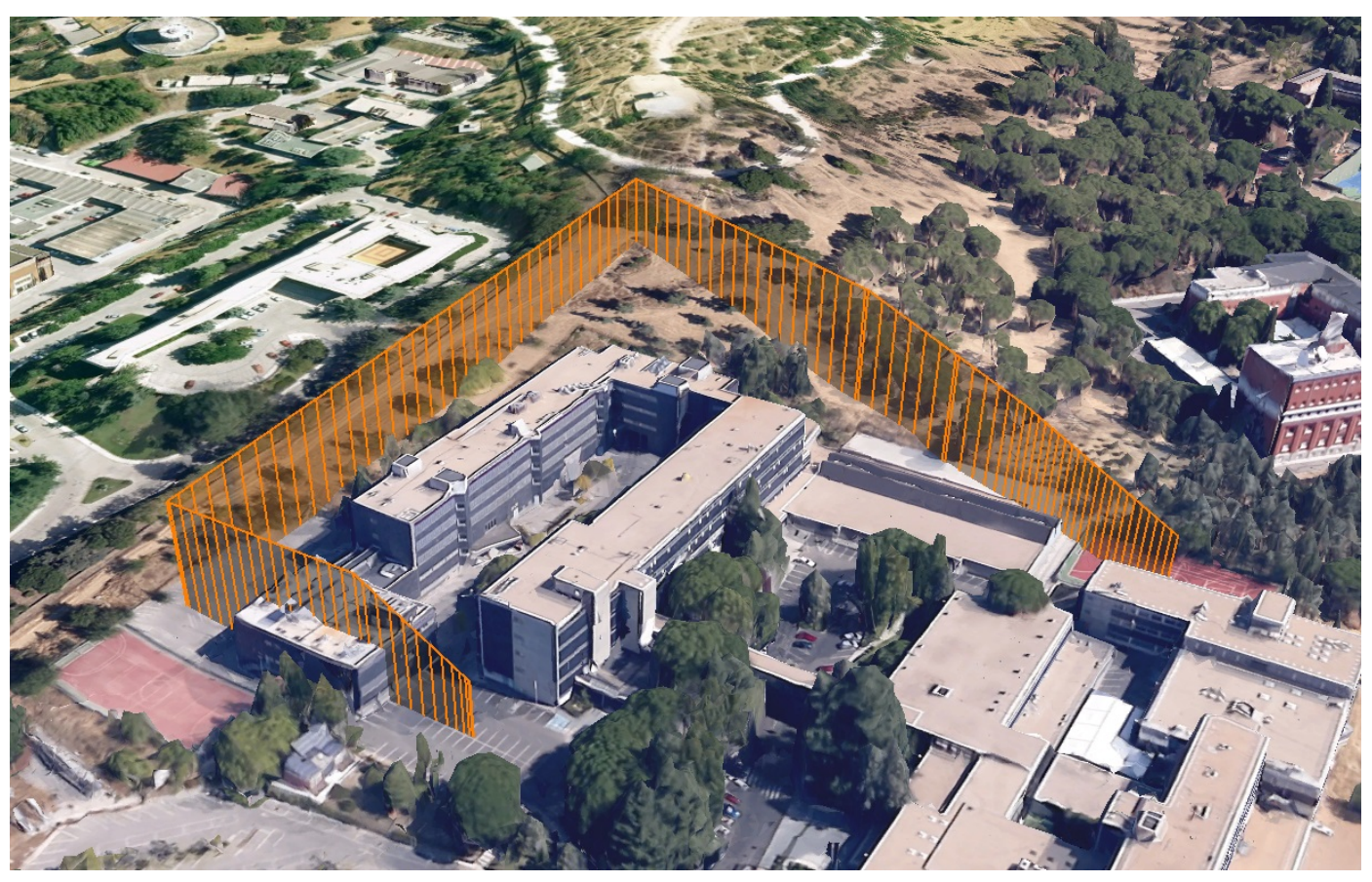

Figure 13.16: Detail of the UA flight (Map data: Google, Europa Technologies).

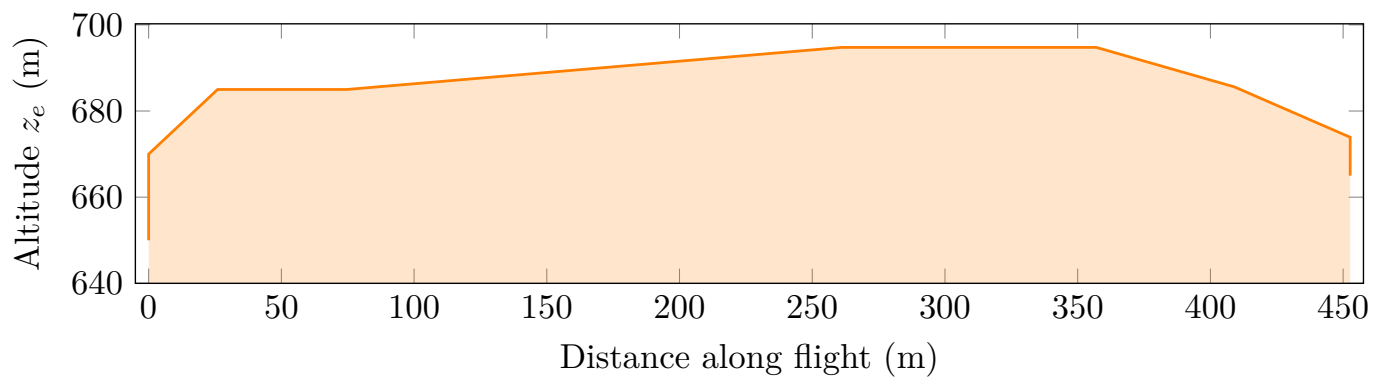

(a) Geometric altitude of trajectory.

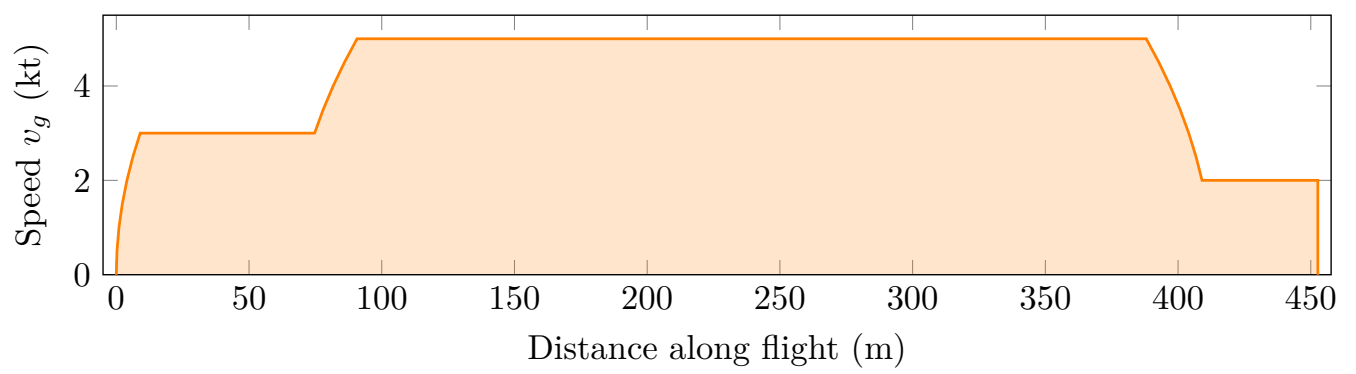

(b) Ground speed of trajectory.

Figure 13.17: Altitude and speed of the flight around the ETSIT. 


\subsection{Final words}

This chapter has illustrated the process of integrating a quadrotor trajectory from an aircraft intent expressed in QR-AIDL. Examples of all the guidance modes available in the QR-AIDL have been provided and, finally, several of these guidance modes have been combined in a single example simulating a flight in a real scenario. All the trajectories shown in this chapter have been computed using a prototype TCE developed to validate the design of the QRAIDL.

The TCE is not the only TLPE described in chapter 11 that can be adapted to be used in UAS instead of ATM. The whole trajectory prediction architecture presented in that chapter could be reused to build a TPE capable of predicting trajectories of UAs.

Additionally, some new TLPEs can be designed to solve specific problems in UAS mission management. They are detailed in the next chapter. 


\section{Chapter 14}

\section{Mission management using formal languages}

This chapter introduces several problems related to the management of a mission, and addresses some of these problems using the formal languages described earlier in this thesis. The concept of TLPEs, or engines, is also used to model some of the functions necessary in mission management.

A civil application of UAS will be studied in this chapter. In particular, an emergency situation in an urban environment is considered, although the same concepts could be applied to different civil and military applications.

Fleets of UA can provide assistance in emergency situations that take place in an urban environment, such as a traffic accident or a fire. Among other things, UAs could perform tasks related to:

- Surveillance. UAs may be equipped with cameras or other sensors that can be used for monitoring static or moving targets, or for searching.

- Transportation. Despite their limited payload, UAs can transport light objects to places that would otherwise be inaccessible or take much longer to reach.

- Actuation. If adequately equipped, UAs can also act on the environment. For example, an UA could be equipped to extinguish small fires, light dark places, or other tasks.

The kind of tasks that a particular UA is able to do depends on the payload of each UA. The hierarchy of languages described in this thesis doesn't allow defining the behaviour of the sensors or actuators that an aircraft may have equipped. This chapter focuses on managing the flights of the UAs (i.e., the trajectories they follow), without further detail on what the UAs (and their sensors/actuators) do at each location. For this purpose, other languages, such as the payload intent description language (PIDL) [74], can be used.

Maza et al. [74] described an architecture for the planning phase of a mission management system. In that architecture, which was briefly described in chapter 4 (see figure 4.2), a trajectory predictor based on the FIDL and the AIDL 


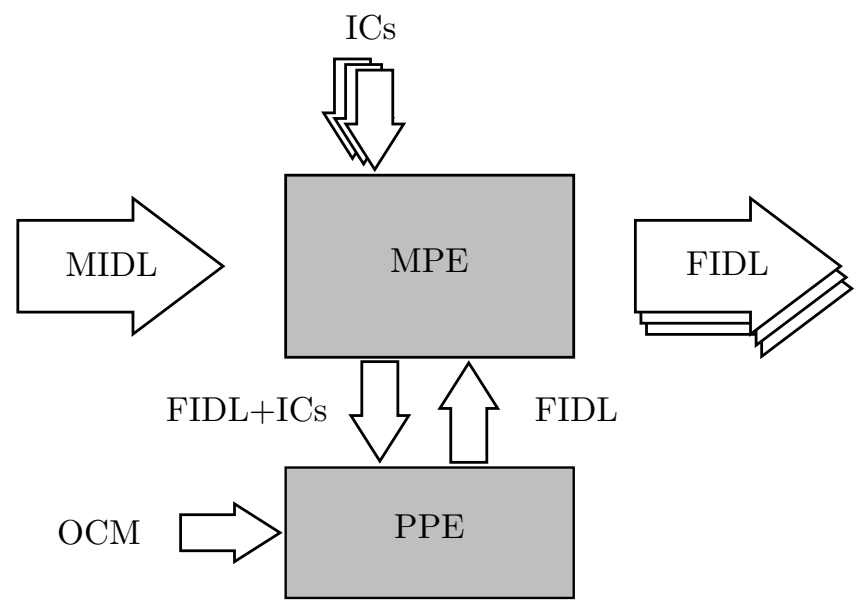

Figure 14.1: The mission planning engine and the path planning engine.

- such as the one described in previous chapters - played an important role. The other key element in that architecture is the mission engine infrastructure (MEI), which contains the mission intent processing engine (MIPE), responsible for translating the input mission intent into a series of flight intents (one for each available aircraft).

This chapter provides some insight on the process of translating mission intent into flight intent. In this chapter, that process is called the mission planning process; and the TLPE that carries out this process, the mission planning engine (MPE). A simplified solution to this problem is provided, which is enough to reveal another key problem in the mission planning process: computing paths between locations. This problem is especially relevant in urban environments, where UAs fly at low altitudes and there are many obstacles that must be avoided. The process that computes these paths can also be seen as a TLPE, the path planning engine (PPE). An efficient algorithm for computing collision-free paths in an urban environment is also presented in this thesis.

Figure 14.1 shows the relationship between the MPE and the PPE. The MPE receives a mission intent expressed in MIDL and the initial conditions of each of the involved aircraft. During the mission planning process, it will be necessary to compute some paths in order to know the associated costs. To obtain the paths, the MPE will provide the PPE with the initial condition and a flight intent containing the initial and final positions of the path to be computed. The PPE makes use of the OCM (which contains information related to the obstacles, forbidden zones, etc. of the environment) to compute a safe path between both locations. This path is provided to the MPE as a flight intent that contains the waypoints and constraints associated to such path. The MPE may require many paths to be computed until it obtains a solution, provided in the form of several flight intents, one for each aircraft.

The next section describes the process carried out at the MPE. Then, section 14.2 is devoted to the development of the algorithm carried out in the PPE. 


\subsection{Translating mission intent into flight intent}

One of the key problems in managing a fleet of UAs is the initial planning of the mission. The initial planning consists on providing flight plans for each of the aircraft in the fleet given a mission specification. This could be seen as a TLPE, the MPE, which receives a mission intent expressed in the MIDL and produces several flight intents expressed in the FIDL.

In the MIDL, missions may include many different tasks, from the simplest to the most complex, with arbitrary constraints and objectives. In this section, a much more constrained problem is tackled, which only constitutes a small subset of what can be expressed in the MIDL. This problem is a generalization of the vehicle routing problem with time windows (VRPTW) [97], where a number of targets must be visited within a specified time interval.

In the following sections, a formal description of the problem is provided, and a mixed integer linear programming (MILP) formulation is presented to solve the problem. A MILP problem is a mathematical optimization problem in which the objective function and the constraints are linear, and where some of the variables are integers and other variables are real numbers. This MILP formulation can be then written into a modelling language (such as AMPL [156]) and computer solvers (such as CPLEX [157]) can be used to obtain solutions to that problem.

The MILP formulation presented here provides an exact solution to the mission planning problem. Obtaining this exact solution is very expensive computationally and, hence, incompatible with real-time optimization. However, alternative MILP formulations could be developed, following similar methods to the one used in [98], at the cost of obtaining sub-optimal solutions.

\subsubsection{Problem formulation}

The problem to be solved in the following sections is formally described here. In this problem, a number of targets must be visited in a specific time window. Some targets are to be visited just once, but others are to be visited periodically. The position of the targets is constant and known. There are a number of aircraft at known initial positions and known performance (speed, operating altitudes, etc.). The aim of the solution is to find paths for every aircraft so that all targets are visited in their time windows and as many times as needed.

This problem is similar to the VRPTW, but there are two important differences:

- Some targets have to be visited periodically, with a maximum interval between visits.

- Aircraft are not required to return to a particular depot after the mission is complete.

In this routing problem, there are $m$ available aircraft, denoted $A_{1}, \ldots, A_{m}$. Each aircraft $A_{i}$ is initially at the $3 \mathrm{D}$ position provided by a vector $\boldsymbol{\zeta}_{0}\left(A_{i}\right)$. In the mission there are also $n$ targets, denoted $T_{1}, \ldots, T_{n}$. Each target $T_{j}$ is at 
the 3D position provided by a vector $\zeta\left(T_{j}\right)$, which is constant throughout the mission. Targets must be visited either during one time window or periodically, with a maximum time interval between visits:

- For targets $T_{j}(1 \leq j \leq n)$ that have to be visited once during a time window defined by the interval $\left[t_{T_{j}}^{\min }, t_{T_{j}}^{\max }\right]$, the constraint

$$
t_{T_{j}}^{\min } \leq t_{T_{j}} \leq t_{T_{j}}^{\max }
$$

is set, where $t_{T_{j}}$ is the time at which target $T_{j}$ is visited.

- For targets $T_{j}(1 \leq j \leq n)$ that have to be visited repeatedly, and where such visits mustn't be more than $I_{T_{j}}$ time apart, the constraint (14.1) applies for the first time $T_{j}$ is visited. For the $l$-th visit $(l \geq 2)$, the constraint

$$
t_{T_{j}}^{l-1} \leq t_{T_{j}}^{l} \leq t_{T_{j}}^{l-1}+I_{T_{j}}
$$

applies, where $t_{T_{j}}^{l}$ is the time at which target $T_{j}$ is visited for the $l$-th time, and $t_{T_{j}}^{1}=t_{T_{j}}$. The periodical visits to $T_{j}$ stop at time $t_{T_{j}}^{\text {end }}$.

As the problem is to be solved in an urban environment, there are some obstacles (buildings, terrain geometry, etc.) on the way, which must be avoided. These obstacles affect the paths that aircraft follow between locations, thus affecting the cost (time, fuel, etc.) of such paths. In this section, it will be assumed that a cost function $\mathcal{C}_{i}$ provides the cost of moving between two locations. In particular, time costs are considered for this problem ${ }^{1}$. An estimation of the time $A_{i}$ takes to go between two locations can be precomputed, as this time can be assumed to be always the same - although it could vary slightly due to changes in weight (fuel, payload) or weather (wind and/or rain can limit UAs' speed). As there may be obstacles in the way, this pre-computation step should also take into account the extra time required to avoid those obstacles (using shortest-path algorithms or other techniques). In section 14.2, the problem of finding paths avoiding all obstacles is addressed.

The solution to the problem is a sequence of tasks assigned to each aircraft. This assignment is denoted $\mathcal{A}$. In this routing problem, the solution consists on the assignment $\mathcal{A}$ that minimizes mission time. The duration of the mission is driven by the time it takes the last aircraft to complete all its tasks, i.e., by the maximum completion time of an aircraft. Thus, the problem is formulated as finding the assignment $\mathcal{A}$ leading to minimizing the maximum completion time, that is:

$$
\text { Minimize: } \max _{i} \mathcal{C}_{i}(\mathcal{A})
$$

where $\mathcal{C}_{i}(\mathcal{A})$ is the time it takes the aircraft $A_{i}$ to complete its tasks (i.e., to visit all the assigned targets).

\footnotetext{
${ }^{1}$ If other costs have to be considered, the formulations provided in this chapter would have to be modified to adapt to them.
} 


\subsubsection{Partial formulation}

The MILP formulation for the problem described above will be constructed incrementally. In a first approach, periodical time windows are ignored. This formulation focuses on the routing problem with time windows at each target. This formulation is based on a similar problem and its corresponding formulation, described in [98].

The problem formulation defines the solution as an assignment $\mathcal{A}$ minimizing the objective function (14.3). However, that expression cannot be included in the MILP formulation, as it is not a linear expression. Equivalently, the solution to the problem can be defined as the assignment $\mathcal{A}$ that minimizes the objective function:

$$
\text { Minimize: } r
$$

subject to

$$
r \geq \mathcal{C}_{i}(\mathcal{A}) \quad \forall i(1 \leq i \leq m)
$$

where $r \in \mathbb{R}$ is a variable of the formulation.

An assignment contains information on which UAs visit which targets, and in what order. This information must be known in order to compute the cost of an assignment. Binary values $a_{i j}^{k} \in\{0,1\}$ indicate whether or not $T_{j}$ is $A_{i}$ 's $k$-th target. The assignment $\mathcal{A}=\left\{a_{i j}^{k}\right\}$ is the set containing all these binary values. There aren't any constraints to the number of targets a single UA can visit, so $k$ is allowed to be as big as $n$ (the total number of targets). This means that a single UA could visit all the targets, or some UAs might not visit any target at all. These two corner cases are rather unlikely, as the optimization criteria tends to distribute targets among all aircraft.

Constraints of the problem must be explicitly stated in the MILP formulation. Each aircraft can only visit one target at a time, which is ensured by adding equation (14.6). Also, $a_{i j}^{k}$ values must be filled in ascending order of $k$, so that the aircraft always have a 'next target' to go to, until they have completed all their tasks. This is forced by equation (14.7). Finally, in this formulation of the problem, all targets are visited exactly once. Equation (14.8) requires this in the MILP formulation.

$$
\begin{array}{rlrl}
\sum_{j=1}^{n} a_{i j}^{k} & \leq 1 & \forall i, k(1 \leq i \leq m, 1 \leq k \leq n) \\
\sum_{j=1}^{n} a_{i j}^{k} \geq \sum_{j=1}^{n} a_{i j}^{k+1} & \forall i, k(1 \leq i \leq m, 1 \leq k \leq n-1) \\
\sum_{i=1}^{m} \sum_{k=1}^{n} a_{i j}^{k} & =1 & \forall j(1 \leq j \leq n)
\end{array}
$$

It is also necessary to define the variables $c_{i}^{k} \in \mathbb{R}$ containing the time invested by each aircraft $A_{i}$ in getting to the $k$-th assigned target from its previous location. A function $\mathcal{C}_{i}$ provides the time required for an aircraft $A_{i}$ to move between locations, but aircraft can also take longer than necessary if desired 
(e.g., not to arrive too soon to a target). This is stated using two equations, (14.9) and (14.10):

$$
\begin{array}{rr}
c_{i}^{1} \geq \sum_{j=1}^{n} a_{i j}^{1} \cdot \mathcal{C}_{i}\left(\boldsymbol{\zeta}_{0}\left(A_{i}\right), \boldsymbol{\zeta}\left(T_{j}\right)\right) & \forall i(1 \leq i \leq m) \\
c_{i}^{k} \geq \sum_{j_{1}=1}^{n} \sum_{j_{2}=1}^{n} a_{i j_{1}}^{k} \cdot a_{i j_{2}}^{k+1} \cdot \mathcal{C}_{i}\left(\boldsymbol{\zeta}\left(T_{j_{1}}\right), \boldsymbol{\zeta}\left(T_{j_{2}}\right)\right) & \forall i, k(1 \leq i \leq m, 1 \leq k \leq n-1)
\end{array}
$$

where $\mathcal{C}_{i}\left(\boldsymbol{\zeta}_{0}\left(A_{i}\right), \boldsymbol{\zeta}\left(T_{j}\right)\right)$ is the time required for $A_{i}$ to go from its initial position to a target $T_{j}$, and $\mathcal{C}_{i}\left(\boldsymbol{\zeta}\left(T_{j_{1}}\right), \boldsymbol{\zeta}\left(T_{j_{2}}\right)\right)$ is the time required for $A_{i}$ to go from target $T_{j_{1}}$ to target $T_{j_{2}}$. Note that, in both constraints, only one of the terms in the sum can be non-zero because of constraint (14.6).

As explained before, the time cost of a path between two locations is assumed to be known at this point. Note equation (14.10) is not linear, as it contains a product of two binary variables. However, some MILP solvers (e.g., CPLEX) can handle this appropriately. Alternatively, constraint (14.10) can be transformed to a linear equation by introducing some auxiliary variables, as it will be shown in the next section.

The $\operatorname{cost} \mathcal{C}_{i}(\mathcal{A})$ can then be defined as:

$$
\mathcal{C}_{i}(\mathcal{A}) \stackrel{\text { def }}{=} \sum_{k=1}^{n} c_{i}^{k} \quad \forall i(1 \leq i \leq m)
$$

The constraints associated with the time windows have to be included in the optimization problem. Constraints (14.12) and (14.13) make sure that targets aren't visited neither too soon nor too late.

$$
\begin{array}{ll}
\sum_{k^{\prime}=1}^{k} c_{i}^{k^{\prime}} \geq \sum_{j=1}^{n} a_{i j}^{k} \cdot t_{T_{j}}^{\min } & \forall i, k(1 \leq i \leq m, 1 \leq k \leq n) \\
\sum_{k^{\prime}=1}^{k} c_{i}^{k^{\prime}} \leq \sum_{j=1}^{n} a_{i j}^{k} \cdot t_{T_{j}}^{\max }+\left(1-\sum_{j=1}^{n} a_{i j}^{k}\right) \cdot B & \forall i, k(1 \leq i \leq m, 1 \leq k \leq n)
\end{array}
$$

Note there is an extra summand at the end of (14.13) that ensures the constraint is met when an aircraft $A_{i}$ doesn't visit any target for a given $k$ (i.e., it has no more scheduled tasks). The constant $B$ must have a value greater than the maximum time for the mission (a good value for $B$ should meet $B>$ $\left.\max _{j} t_{T_{j}}^{\max }\right)$.

The objective function (14.4) and constraints (14.5)-(14.13) form the MILP formulation. This formulation is summarized next. 


\section{Minimize: $r$}

subject to

$$
\begin{aligned}
& r \geq \sum_{k=1}^{n} c_{i}^{k} \quad \forall i(1 \leq i \leq m) \\
& \sum_{j=1}^{n} a_{i j}^{k} \leq 1 \quad \forall i, k(1 \leq i \leq m, 1 \leq k \leq n) \\
& \sum_{j=1}^{n} a_{i j}^{k} \geq \sum_{j=1}^{n} a_{i j}^{k+1} \quad \forall i, k(1 \leq i \leq m, 1 \leq k \leq n-1) \\
& \sum_{i=1}^{m} \sum_{k=1}^{n} a_{i j}^{k}=1 \quad \forall j(1 \leq j \leq n) \\
& c_{i}^{1} \geq \sum_{j=1}^{n} a_{i j}^{1} \cdot \mathcal{C}_{i}\left(\boldsymbol{\zeta}_{0}\left(A_{i}\right), \boldsymbol{\zeta}\left(T_{j}\right)\right) \\
& \forall i(1 \leq i \leq m) \\
& c_{i}^{k} \geq \sum_{j_{1}=1}^{n} \sum_{j_{2}=1}^{n} a_{i j_{1}}^{k} \cdot a_{i j_{2}}^{k+1} \cdot \mathcal{C}_{i}\left(\boldsymbol{\zeta}\left(T_{j_{1}}\right), \boldsymbol{\zeta}\left(T_{j_{2}}\right)\right) \\
& \forall i, k(1 \leq i \leq m, 1 \leq k \leq n-1) \\
& \sum_{k^{\prime}=1}^{k} c_{i}^{k^{\prime}} \geq \sum_{j=1}^{n} a_{i j}^{k} \cdot t_{T_{j}}^{\min } \quad \forall i, k(1 \leq i \leq m, 1 \leq k \leq n) \\
& \sum_{k^{\prime}=1}^{k} c_{i}^{k^{\prime}} \leq \sum_{j=1}^{n} a_{i j}^{k} \cdot t_{T_{j}}^{\max }+\left(1-\sum_{j=1}^{n} a_{i j}^{k}\right) \cdot B \quad \forall i, k(1 \leq i \leq m, 1 \leq k \leq n)
\end{aligned}
$$

The problem solved in this section is only a partial formulation of the complete mission planning problem. As stated in the beginning of this section, periodical time windows from the problem description in section 14.1.1 have been omitted.

\subsubsection{Alternative partial formulation}

Adding the periodical time windows to the partial formulation requires reformulating many of its constraints. This section includes a different MILP formulation that solves the same problem as in the previous section, that is, it doesn't include the constraints related to periodical time windows but it prepares the way for its inclusion in the next section.

In this formulation, the assignment is still expressed as a set of binary values 
$\mathcal{A}=\left\{a_{i j}^{k}\right\}$ that indicate whether $T_{j}$ is $A_{i}$ 's $k$-th target. Constraints (14.6), (14.7), and (14.8), defined in the previous formulation, also apply to those values. However, variables $c_{i}^{k} \in \mathbb{R}$ have a different definition: in this case, $c_{i}^{k}$ contains the time at which aircraft $A_{i}$ arrives to its $k$-th target. The constraints related to $c_{i}^{k}$ must be reformulated.

In the previous formulation, constraint (14.10) included a product of two binary values, which makes the formulation non-linear. To make it linear, the solution proposed in [98] is used. Variables $y_{i}^{k}\left(j_{1}, j_{2}\right) \in\{0,1\}$ are introduced, which indicate whether the aircraft $A_{i}$ travels from target $T_{j_{1}}$ to target $T_{j_{2}}$ between times $k$ and $k+1$. The objective is to make $y_{i}^{k}\left(j_{1}, j_{2}\right)=a_{i j_{1}}^{k} \cdot a_{i j_{2}}^{k+1}$, but without explicitly including the product. This can be done by including the constraint:

$$
\begin{aligned}
& a_{i j_{1}}^{k}+a_{i j_{2}}^{k+1}=2 \cdot y_{i}^{k}\left(j_{1}, j_{2}\right)+\rho_{i}^{k}\left(j_{1}, j_{2}\right) \\
& \quad \forall i, j_{1}, j_{2}, k\left(1 \leq i \leq m, 1 \leq j_{1} \leq n, 1 \leq j_{2} \leq n, 1 \leq k \leq n-1\right)
\end{aligned}
$$

where $\rho_{i}^{k}\left(j_{1}, j_{2}\right) \in[0,1]$ are auxiliary variables.

The time at which an aircraft $A_{i}$ arrives to its $k$-th target cannot be lower than the minimum time required to travel to all the previous targets, from the first to the $k$-th. Constraint (14.15) explicitly establishes this condition. The first sum contains the cost of travelling to the first target from the initial conditions, and the second sum contains the cost of travelling between all the previous targets, until the $k$-th (if $k=1$, this sum is always zero).

$$
\begin{array}{r}
c_{i}^{k} \geq \sum_{j=1}^{n} a_{i j}^{1} \cdot \mathcal{C}_{i}\left(\boldsymbol{\zeta}_{0}\left(A_{i}\right), \boldsymbol{\zeta}\left(T_{j_{1}}\right)\right)+\sum_{k^{\prime}=1}^{k-1} \sum_{j_{1}=1}^{n} \sum_{j_{2}=1}^{n} y_{i}^{k^{\prime}}\left(j_{1}, j_{2}\right) \cdot \mathcal{C}_{i}\left(\boldsymbol{\zeta}\left(T_{j_{1}}\right), \boldsymbol{\zeta}\left(T_{j_{2}}\right)\right) \\
\forall i, k(1 \leq i \leq m, 1 \leq k \leq n) \quad(14.15)
\end{array}
$$

Also, the time required by an aircraft $A_{i}$ for going from one target $T_{j_{1}}$ to the next target $T_{j_{2}}$ can't be lower than $\mathcal{C}_{i}\left(\boldsymbol{\zeta}\left(T_{j_{1}}\right), \boldsymbol{\zeta}\left(T_{j_{2}}\right)\right)$. This is ensured by constraint (14.16).

$$
\begin{aligned}
c_{i}^{k+1} \geq c_{i}^{k}+\sum_{j_{1}=1}^{n} \sum_{j_{2}=1}^{n} y_{i}^{k}\left(j_{1}, j_{2}\right) \cdot \mathcal{C}_{i}\left(\boldsymbol{\zeta}\left(T_{j_{1}}\right), \boldsymbol{\zeta}\left(T_{j_{2}}\right)\right) \\
\quad \forall i, k(1 \leq i \leq m, 1 \leq k \leq n-1)
\end{aligned}
$$

The constraints related to the time windows are analogous to (14.12) and (14.13), slightly modified to adapt to the new definitions of $c_{i}^{k}$.

$$
\begin{array}{ll}
c_{i}^{k} \geq \sum_{j=1}^{n} a_{i j}^{k} \cdot t_{T_{j}}^{\min } & \forall i, k(1 \leq i \leq m, 1 \leq k \leq n) \\
c_{i}^{k} \leq \sum_{j=1}^{n} a_{i j}^{k} \cdot t_{T_{j}}^{\max }+\left(1-\sum_{j=1}^{n} a_{i j}^{k}\right) \cdot B & \forall i, k(1 \leq i \leq m, 1 \leq k \leq n)
\end{array}
$$

Finally, the time an aircraft $A_{i}$ needs to complete all the tasks in the assignment, $\mathcal{C}_{i}(\mathcal{A})$, is defined as:

$$
\mathcal{C}_{i}(\mathcal{A}) \stackrel{\text { def }}{=} c_{i}^{n} \quad \forall i(1 \leq i \leq m)
$$


This alternate formulation is based on the same objective function (14.4) and constraints (14.5)-(14.8) and (14.14)-(14.19). All these equations in this formulation are summarized below:

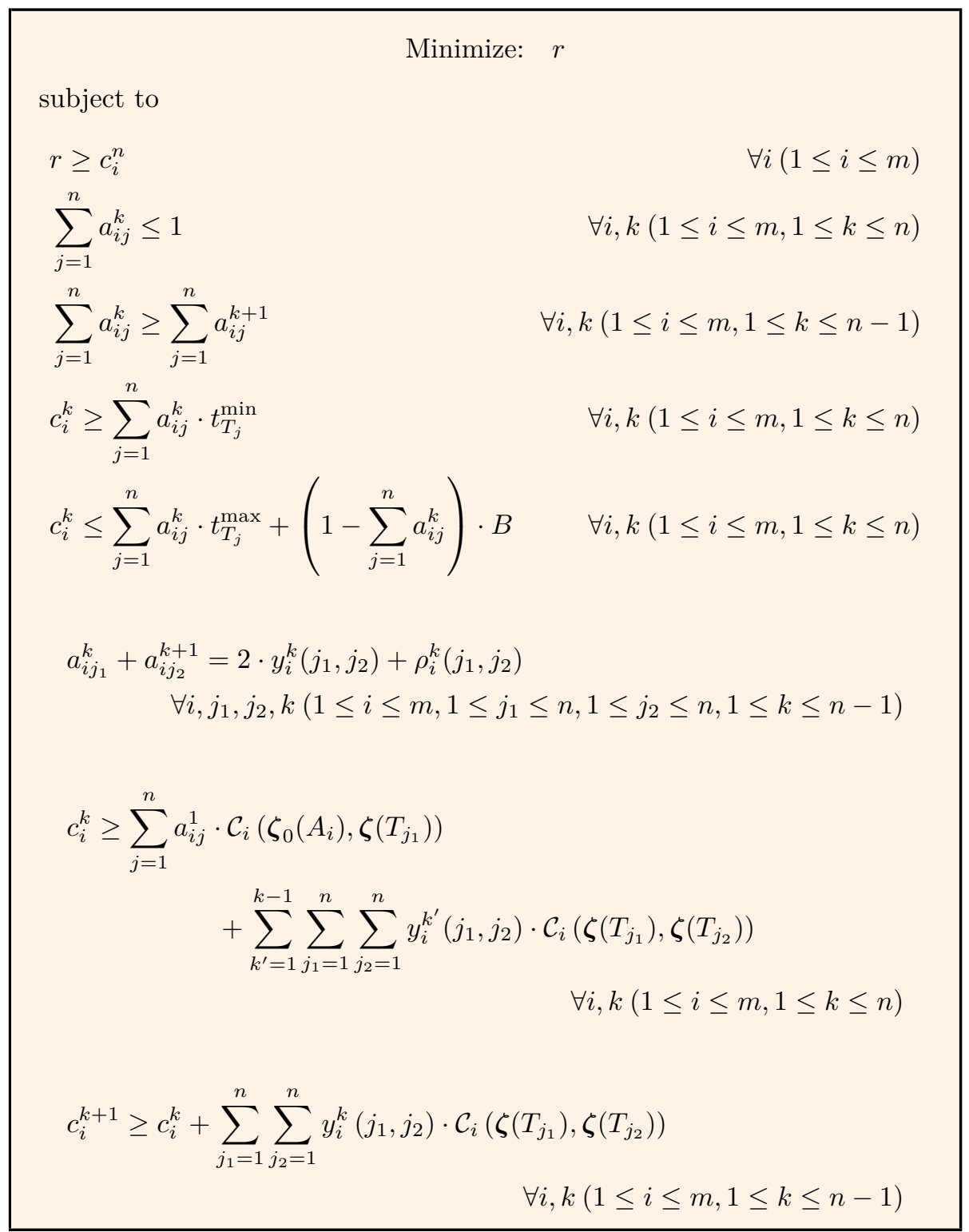

This MILP formulation still doesn't take into account periodical time windows, which are finally included in the complete formulation described in the next section.

\subsubsection{Complete formulation}

A complete MILP formulation of the problem is presented in this section. The periodical time windows, that is, targets that must be visited periodically, 
must be now considered. Therefore, some targets will be visited more than once. Without loss of generality, it is be assumed that the targets with periodical time windows are $T_{1}, \ldots, T_{n^{\prime}}\left(n^{\prime} \leq n\right)$.

In the previous formulation, the order in which one aircraft visited different targets was specified in the assignment. An index $k$ was used to indicate the order in which an aircraft would traverse the assigned targets. In this case, the complete formulation also requires keeping track of what order one target is visited by different aircraft. Another index $l$ is used to number each time a target is visited by any aircraft. Binary values $e_{i j}^{k l} \in\{0,1\}$ indicate whether $A_{i}$ 's $k$-th target corresponds to the $l$-th visit to $T_{j}$. Assignments $\mathcal{A}$ contain all these binary values, $\mathcal{A}=\left\{e_{i j}^{k l}\right\}$.

An upper bound to $l$, denoted $N$, must be defined. This upper bound limits the maximum number of times that a target can be visited, so it may affect the solvability of the MILP formulation. It was found that a suitable value for $N$ is:

$$
N=2 \cdot \max _{j}\left\lceil\frac{t_{T_{j}}^{\mathrm{end}}-t_{T_{j}}^{\min }}{I_{T_{j}}}\right\rceil
$$

This means that no target will be visited more than twice as many times as necessary (if there were no other constraints). As there are $n$ targets, and each target is visited at most $N$ times, any assignment will contain at most $N \cdot n$ visits. In the worst case, one aircraft would have to pay all the visits, and therefore the upper bound to $k$ is $N \cdot n$.

With $e_{i j}^{k l}$ having four indices, the binary values used in this formulation can be cumbersome to work with. To deal with them more easily the following two aliases are defined:

$$
\begin{array}{rlrl}
a_{i j}^{k} & =\sum_{l=1}^{N} e_{i j}^{k l} & \forall i, j, k(1 \leq i \leq m, 1 \leq j \leq n, 1 \leq k \leq N \cdot n) \\
b_{i j}^{l}=\sum_{k=1}^{N \cdot n} e_{i j}^{k l} & \forall i, j, l(1 \leq i \leq m, 1 \leq j \leq n, 1 \leq l \leq N)
\end{array}
$$

where $a_{i j}^{k}$ indicates whether $T_{j}$ is $A_{i}$ 's $k$-th target (exactly as in the previous formulation), and $b_{i j}^{l}$ indicates whether the $l$-th visit to $T_{j}$ is paid by $A_{i}$. It can also be observed that $e_{i j}^{k l}=a_{i j}^{k} \cdot b_{i j}^{l}$.

Aircraft can still only visit one target at a time, and the $a_{i j}^{k}$ values (i.e., $e_{i j}^{k l}$ values) must be filled in ascending order of $k$. Therefore, constraints (14.6) and (14.7) also apply in the complete formulation. Additionally, analogous constraints have to be defined for the targets: Each target can only be visited by one target at a time, and the $b_{i j}^{l}$ values (i.e., $e_{i j}^{k l}$ values) must be filled in ascending order of $l$. This is ensured by equations (14.22) and (14.23), respectively:

$$
\begin{array}{lr}
\sum_{i=1}^{m} b_{i j}^{l} \leq 1 & \forall j, l(1 \leq j \leq n, 1 \leq l \leq N) \\
\sum_{i=1}^{m} b_{i j}^{l} \geq \sum_{i=1}^{m} b_{i j}^{l+1} & \forall j, l(1 \leq j \leq n, 1 \leq l \leq N-1)
\end{array}
$$


It is also necessary to keep more information related to the times at which aircraft reach each target. Variables $c_{i j}^{k l} \in \mathbb{R}$ contain the time each aircraft $A_{i}$ reaches its $k$-th assigned target $T_{j}$, corresponding to the $l$-th visit to that target. This time must be zero if the event doesn't occur (i.e., if $e_{i j}^{k l}=0$, then $c_{i j}^{k l}=0$ ), as described by constraint (14.24).

$$
\begin{array}{rl}
c_{i j}^{k l} \leq e_{i j}^{k l} \cdot B & B, j, k, l(1 \leq i \leq m, 1 \leq j \leq n, 1 \leq k \leq N \cdot n, 1 \leq l \leq N)
\end{array}
$$

where $B$ is a sufficiently large constant, a time greater than the duration of the whole mission (a good value for $B$ should meet $B>\max _{j} t_{T_{j}}^{\text {end }}$ and $B>$ $\left.\max _{j} t_{T_{j}}^{\max }\right)$.

Several other constraints apply to the variables $c_{i j}^{k l}$. The time at which an aircraft $A_{i}$ reaches its $k$-th assigned target must be, at least, the time required to travel to all the previous targets, from the first to the $k$-th. In other words, $c_{i j}^{k l}$ must be greater or equal to the time required to travel from its initial position to the position of the first target, then the second target, etc., until the $k$-th target. This is expressed in constraint (14.25).

$$
\begin{gathered}
c_{i j}^{k l} \geq\left(\sum_{j_{1}=1}^{n} a_{i j_{1}}^{1} \cdot \mathcal{C}_{i}\left(\boldsymbol{\zeta}_{0}\left(A_{i}\right), \boldsymbol{\zeta}\left(T_{j_{1}}\right)\right)\right) \cdot e_{i j}^{k l} \\
\quad+\left(\sum_{k^{\prime}=1}^{k-1} \sum_{j_{1}=1}^{n} \sum_{j_{2}=1}^{n} y_{i}^{k^{\prime}}\left(j_{1}, j_{2}\right) \cdot \mathcal{C}_{i}\left(\boldsymbol{\zeta}\left(T_{j_{1}}\right), \boldsymbol{\zeta}\left(T_{j_{2}}\right)\right)\right) \cdot e_{i j}^{k l} \\
\forall i, j, k, l(1 \leq i \leq m, 1 \leq j \leq n, 1 \leq k \leq N \cdot n, 1 \leq l \leq N)
\end{gathered}
$$

The first sum in constraint (14.25) contains the cost of travelling from the initial position to the first target, and the second sum includes the remaining costs of travelling between targets. Both sums are multiplied by $e_{i j}^{k l}$ so that the resulting time is zero if the aircraft doesn't visit the corresponding target in that specific order. The constraint also makes use of variables $y_{i}^{k}\left(j_{1}, j_{2}\right)$ that indicate whether the aircraft $A_{i}$ travels from target $T_{j_{1}}$ to target $T_{j_{2}}$ between times $k$ and $k+1$. These variables are analogous to those used in the previous formulation. An additional constraint must be added:

$$
\begin{aligned}
& a_{i j_{1}}^{k}+a_{i j_{2}}^{k+1}=2 \cdot y_{i}^{k}\left(j_{1}, j_{2}\right)+\rho_{i}^{k}\left(j_{1}, j_{2}\right) \\
& \quad \forall i, j_{1}, j_{2}, k\left(1 \leq i \leq m, 1 \leq j_{1} \leq n, 1 \leq j_{2} \leq n, 1 \leq k \leq N \cdot n-1\right)
\end{aligned}
$$

where $y_{i}^{k}\left(j_{1}, j_{2}\right) \in\{0,1\}$ and $\rho_{i}^{k}\left(j_{1}, j_{2}\right) \in[0,1]$.

The last constraint related to defining $c_{i j}^{k l}$ indicates that the time required by an aircraft $A_{i}$ in going from one target $T_{j_{1}}$ to the next target $T_{j_{2}}$ can't be lower than $\mathcal{C}_{i}\left(\boldsymbol{\zeta}\left(T_{j_{1}}\right), \boldsymbol{\zeta}\left(T_{j_{2}}\right)\right)$.

$$
\begin{gathered}
c_{i j}^{k+1 l} \geq \sum_{j_{1}=1}^{n} \sum_{l^{\prime}=1}^{n} c_{i j_{1}}^{k l^{\prime}}+\sum_{j_{1}=1}^{n} y_{i}^{k}\left(j_{1}, j\right) \cdot \mathcal{C}_{i}\left(\boldsymbol{\zeta}\left(T_{j_{1}}\right), \boldsymbol{\zeta}\left(T_{j_{2}}\right)\right)-\left(1-e_{i j}^{k+1 l}\right) \cdot B \\
\forall i, j, k, l(1 \leq i \leq m, 1 \leq j \leq n, 1 \leq k \leq N \cdot n-1,1 \leq l \leq N) \quad
\end{gathered}
$$


The constraint above only makes sense when $e_{i j}^{k+1 l} \neq 0$. In order to ensure that the constraint is met when $e_{i j}^{k+1 l}=0$, the subtrahend at the end of (14.27) is added.

Now that the values of $c_{i j}^{k l}$ have been appropriately defined, it is time to define the constraints that will ensure that the assignment fulfils all the required tasks, that is, all targets are visited when required (and as many times as required). Another alias will be defined to make such constraints more readable. The time at which a target $T_{j}$ is visited for the $l$-th time is denoted $t_{T_{j}}^{l}$ and defined as:

$$
t_{T_{j}}^{l}=\sum_{i=1}^{m} \sum_{k=1}^{N \cdot n} c_{i j}^{k l} \quad \forall j, l(1 \leq j \leq n, 1 \leq l \leq N)
$$

These visits must occur in chronological order:

$$
t_{T_{j}}^{l} \leq t_{T_{j}}^{l+1}+\left(1-\sum_{i=1}^{m} b_{i j}^{l+1}\right) \cdot B \quad \forall j, l(1 \leq j \leq n, 1 \leq l \leq N-1)
$$

where the summand at the end makes the constraint trivially met if the target $T_{j}$ is visited less than $l+1$ times.

All targets with periodical time windows $T_{j}\left(1 \leq j \leq n^{\prime}\right)$ must be visited at least once. This is ensured by constraint (14.30). On the other hand, the rest of the targets $T_{j}\left(m^{\prime}+1 \leq j \leq n\right)$ must be visited exactly once, which is ensured by constraint (14.31).

$$
\begin{array}{lrl}
\sum_{i=1}^{m} \sum_{k=1}^{N \cdot n} a_{i j}^{k} \geq 1 & \forall j\left(1 \leq j \leq n^{\prime}\right) \\
\sum_{i=1}^{m} \sum_{k=1}^{N \cdot n} a_{i j}^{k}=1 & \forall j\left(n^{\prime}+1 \leq j \leq n\right)
\end{array}
$$

The first visit to a target must happen during the corresponding time window, as in the previous formulations. Two constraints, (14.32) and (14.33), are used to ensure this.

$$
\begin{array}{r}
\sum_{j=1}^{n} \sum_{l=1}^{N} c_{i j}^{k l} \geq \sum_{j=1}^{n} e_{i j}^{k 1} \cdot t_{T_{j}}^{\min } \\
\forall i, k(1 \leq i \leq m, 1 \leq k \leq N \cdot n) \\
\sum_{j=1}^{n} \sum_{l=1}^{N} c_{i j}^{k l} \leq \sum_{j=1}^{n} e_{i j}^{k 1} \cdot t_{T_{j}}^{\max }+\left(\begin{array}{r}
1-\sum_{j=1}^{n} e_{i j}^{k 1} \\
\forall i, k(1 \leq i \leq m, 1 \leq k \leq N \cdot n)
\end{array}\right.
\end{array}
$$

where, again, the last summand in constraint (14.33) ensures this constraint is met when the aircraft that visits $T_{j}$ for the first time is still unassigned.

So far, the constraints included in this section are almost analogous to the constraints in the partial formulation in the previous section. The alternative 
definition of some of the variables makes the constraints, in some cases, far more complicated. However, this increase in the number and complexity of constraints and variables allows defining what was missing in the previous formulation: some targets must be visited periodically.

As stated before, it is assumed that the targets with periodical time windows are $T_{1}, \ldots, T_{n^{\prime}}\left(n^{\prime} \leq n\right)$. One constraint states the maximum time that may elapse between two visits to the same target:

$$
\begin{aligned}
& t_{T_{j}}^{l}+I_{T_{j}} \geq t_{T_{j}}^{l+1}-\left(1-\sum_{i=1}^{m} b_{i j}^{l+1}\right) \cdot B \\
& \forall j, l\left(1 \leq j \leq n^{\prime}, 1 \leq l \leq N-1\right)
\end{aligned}
$$

where the subtrahend at the end of (14.34) ensures that the constraint is met if the target isn't visited $l+1$ times.

The only requirement that remains to be specified as a constraint is the need for targets $T_{1}, \ldots, T_{n^{\prime}}$ to be visited repeatedly until time $t_{T_{j}}^{\text {end }}$ is reached. In this case, the last visit to $T_{j}$ may not occur before $t_{T_{j}}^{\text {end }}-I_{T_{j}}$, as specified in constraints (14.35) and (14.36). Two equations are necessary, one contemplating the case where the target is visited less than $N$ times, and another case where it is visited exactly $N$ times:

$$
\begin{array}{lr}
t_{T_{j}}^{l}+I_{T_{j}} \geq t_{T_{j}}^{\mathrm{end}}-\left(\sum_{i=1}^{m} b_{i j}^{l+1}\right) \cdot B & \forall j, l\left(1 \leq j \leq n^{\prime}, 1 \leq l \leq N-1\right) \\
t_{T_{j}}^{N}+I_{T_{j}} \geq t_{T_{j}}^{\mathrm{end}}-\left(1-\sum_{i=1}^{m} b_{i j}^{N}\right) \cdot B & \forall j\left(1 \leq j \leq n^{\prime}\right)
\end{array}
$$

where the subtrahend at the end of (14.35) ensures the constraint is met if the target hasn't been visited exactly $l$ times; and the subtrahend at the end of (14.36) ensures it is met if the target hasn't been visited exactly $N$ times.

With all the constraints specified, it is time to define the optimization target. The optimization target is similar to the one in the previous formulation but, since some variables have a different definition, it has varied slightly. In this case, the solution to the problem can be defined as the assignment $\mathcal{A}$ that minimizes:

$$
\text { Minimize: } r
$$

subject to

$$
r \geq c_{i j}^{k l} \quad \forall i, j, k, l(1 \leq i \leq m, 1 \leq j \leq n, 1 \leq k \leq N \cdot n, 1 \leq l \leq N)
$$

The MILP formulation provided in this chapter provides an optimal solution to the problem of mission planning formulated in section 14.1.1. However, the number of variables required by this formulation is in $O\left(n^{2} N^{2} m\right)$, so it grows rapidly with the number of available aircraft, the number of targets, and even the duration of the mission. As the number of variables grows, so does the time 
required to compute a solution to this mission planning problem. Therefore, this formulation is not suitable for real-time use.

A summary of the equations in this formulation is included next.

\section{Minimize: $r$}

subject to

$r \geq c_{i j}^{k l} \quad \forall i, j, k, l(1 \leq i \leq m, 1 \leq j \leq n, 1 \leq k \leq N \cdot n, 1 \leq l \leq N)$

$a_{i j}^{k}=\sum_{l=1}^{N} e_{i j}^{k l} \quad \forall i, j, k(1 \leq i \leq m, 1 \leq j \leq n, 1 \leq k \leq N \cdot n)$

$b_{i j}^{l}=\sum_{k=1}^{N \cdot n} e_{i j}^{k l} \quad \forall i, j, l(1 \leq i \leq m, 1 \leq j \leq n, 1 \leq l \leq N)$

$\sum_{j=1}^{n} a_{i j}^{k} \leq 1 \quad \forall i, k(1 \leq i \leq m, 1 \leq k \leq N \cdot n)$

$\sum_{j=1}^{n} a_{i j}^{k} \geq \sum_{j=1}^{n} a_{i j}^{k+1} \quad \forall i, k(1 \leq i \leq m, 1 \leq k \leq N \cdot n-1)$

$\sum_{i=1}^{m} b_{i j}^{l} \leq 1 \quad \forall j, l(1 \leq j \leq n, 1 \leq l \leq N)$

$\sum_{i=1}^{m} b_{i j}^{l} \geq \sum_{i=1}^{m} b_{i j}^{l+1} \quad \forall j, l(1 \leq j \leq n, 1 \leq l \leq N-1)$

$\sum_{i=1}^{m} \sum_{k=1}^{N \cdot n} a_{i j}^{k} \geq 1 \quad \forall j\left(1 \leq j \leq n^{\prime}\right)$

$\sum_{i=1}^{m} \sum_{k=1}^{N \cdot n} a_{i j}^{k}=1 \quad \forall j\left(n^{\prime}+1 \leq j \leq n\right)$

$c_{i j}^{k l} \leq e_{i j}^{k l} \cdot B$

$\forall i, j, k, l(1 \leq i \leq m, 1 \leq j \leq n, 1 \leq k \leq N \cdot n, 1 \leq l \leq N)$

$a_{i j_{1}}^{k}+a_{i j_{2}}^{k+1}=2 \cdot y_{i}^{k}\left(j_{1}, j_{2}\right)+\rho_{i}^{k}\left(j_{1}, j_{2}\right)$

$\forall i, j_{1}, j_{2}, k\left(1 \leq i \leq m, 1 \leq j_{1} \leq n, 1 \leq j_{2} \leq n, 1 \leq k \leq N \cdot n-1\right)$ 


$$
\begin{aligned}
& c_{i j}^{k l} \geq\left(\sum_{j_{1}=1}^{n} a_{i j_{1}}^{1} \cdot \mathcal{C}_{i}\left(\boldsymbol{\zeta}_{0}\left(A_{i}\right), \boldsymbol{\zeta}\left(T_{j_{1}}\right)\right)\right) \cdot e_{i j}^{k l} \\
& +\left(\sum_{k^{\prime}=1}^{k-1} \sum_{j_{1}=1}^{n} \sum_{j_{2}=1}^{n} y_{i}^{k^{\prime}}\left(j_{1}, j_{2}\right) \cdot \mathcal{C}_{i}\left(\boldsymbol{\zeta}\left(T_{j_{1}}\right), \boldsymbol{\zeta}\left(T_{j_{2}}\right)\right)\right) \cdot e_{i j}^{k l} \\
& \forall i, j, k, l(1 \leq i \leq m, 1 \leq j \leq n, 1 \leq k \leq N \cdot n, 1 \leq l \leq N) \\
& c_{i j}^{k+1 l} \geq \sum_{j_{1}=1}^{n} \sum_{l^{\prime}=1}^{n} c_{i j_{1}}^{k l^{\prime}}+\sum_{j_{1}=1}^{n} y_{i}^{k}\left(j_{1}, j\right) \cdot \mathcal{C}_{i}\left(\boldsymbol{\zeta}\left(T_{j_{1}}\right), \boldsymbol{\zeta}\left(T_{j_{2}}\right)\right)-\left(1-e_{i j}^{k+1 l}\right) \cdot B \\
& \forall i, j, k, l(1 \leq i \leq m, 1 \leq j \leq n, 1 \leq k \leq N \cdot n-1,1 \leq l \leq N) \\
& t_{T_{j}}^{l}=\sum_{i=1}^{m} \sum_{k=1}^{N \cdot n} c_{i j}^{k l} \quad \forall j, l(1 \leq j \leq n, 1 \leq l \leq N) \\
& t_{T_{j}}^{l} \leq t_{T_{j}}^{l+1}+\left(1-\sum_{i=1}^{m} b_{i j}^{l+1}\right) \cdot B \quad \forall j, l(1 \leq j \leq n, 1 \leq l \leq N-1) \\
& \sum_{j=1}^{n} \sum_{l=1}^{N} c_{i j}^{k l} \geq \sum_{j=1}^{n} e_{i j}^{k 1} \cdot t_{T_{j}}^{\min } \\
& \forall i, k(1 \leq i \leq m, 1 \leq k \leq N \cdot n) \\
& \sum_{j=1}^{n} \sum_{l=1}^{N} c_{i j}^{k l} \leq \sum_{j=1}^{n} e_{i j}^{k 1} \cdot t_{T_{j}}^{\max }+\left(1-\sum_{j=1}^{n} e_{i j}^{k 1}\right) \cdot B \\
& \forall i, k(1 \leq i \leq m, 1 \leq k \leq N \cdot n) \\
& t_{T_{j}}^{l}+I_{T_{j}} \geq t_{T_{j}}^{l+1}-\left(1-\sum_{i=1}^{m} b_{i j}^{l+1}\right) \cdot B \\
& \forall j, l\left(1 \leq j \leq n^{\prime}, 1 \leq l \leq N-1\right) \\
& t_{T_{j}}^{l}+I_{T_{j}} \geq t_{T_{j}}^{\text {end }}-\left(\sum_{i=1}^{m} b_{i j}^{l+1}\right) \cdot B \quad \forall j, l\left(1 \leq j \leq n^{\prime}, 1 \leq l \leq N-1\right) \\
& t_{T_{j}}^{N}+I_{T_{j}} \geq t_{T_{j}}^{\text {end }}-\left(1-\sum_{i=1}^{m} b_{i j}^{N}\right) \cdot B \quad \forall j\left(1 \leq j \leq n^{\prime}\right)
\end{aligned}
$$

Providing a formal formulation to this problem, however, has revealed other important factors in mission planning, such as the computation of the cost of travelling between two locations during the mission. In this section, it was 
assumed that a function $\mathcal{C}_{i}$ provided such costs. Section 14.2 will focus on the development of a fast method to compute near-optimal paths and their associated costs.

\subsubsection{Example}

Using the complete formulation provided in the previous section, an example mission is planned. This section provides the details of the example mission and the resulting assignment and costs.

The complete formulation of the problem has been written in AMPL [156], a modelling language for optimization problems. Then, a MILP solver, CPLEX [157], can use the AMPL model and the mission information to compute an optimal solution to the problem.

The example mission provided in this section comprises five targets (three of which must be visited periodically) and two aircraft. Therefore, $n=6, n^{\prime}=3$, and $m=2$. Additionally, all the time windows, intervals, and costs must be provided.

The time windows for each target $T_{j}(j \in\{1,2,3,4,5,6\})$ constrain the times at which those targets must be visited for the first time. The time windows in the example presented here are contained in table 14.1.

\begin{tabular}{l|rrrrrr} 
& \multicolumn{7}{|c}{$j$} \\
& 1 & 2 & 3 & 4 & \multicolumn{1}{c}{5} & 6 \\
\hline$t_{T_{j}}^{\min }$ & $80 \mathrm{~s}$ & $0 \mathrm{~s}$ & $140 \mathrm{~s}$ & $150 \mathrm{~s}$ & $30 \mathrm{~s}$ & $180 \mathrm{~s}$ \\
$t_{T_{j}}^{\max }$ & $110 \mathrm{~s}$ & $50 \mathrm{~s}$ & $165 \mathrm{~s}$ & $200 \mathrm{~s}$ & $130 \mathrm{~s}$ & $300 \mathrm{~s}$
\end{tabular}

Table 14.1: Time windows in example mission.

After they have been visited for the first time during the corresponding time window, targets $T_{1}, T_{2}$, and $T_{3}$ must be visited periodically for a certain amount of time. An interval $I_{T_{j}}$ defines, for each target $T_{j}(j \in\{1,2,3\})$, the maximum time between visits. A time $t_{T_{j}}^{\text {end }}$ determines the time at which periodical visits end. The corresponding values are included in table 14.2.

\begin{tabular}{l|ccc} 
& \multicolumn{3}{|c}{$j$} \\
& 1 & 2 & 3 \\
\hline$I_{T_{j}}$ & $100 \mathrm{~s}$ & $180 \mathrm{~s}$ & $100 \mathrm{~s}$ \\
$t_{T_{j}}^{\text {end }}$ & $300 \mathrm{~s}$ & $400 \mathrm{~s}$ & $380 \mathrm{~s}$
\end{tabular}

Table 14.2: Intervals between visits and periodical visits ending times.

Lastly, the time costs of travelling between locations are provided. Table 14.3 contains the costs of travelling between any two targets $T_{j_{1}}$ and $T_{j_{2}}$, denoted $\mathcal{C}_{i}\left(\boldsymbol{\zeta}\left(T_{j_{1}}\right), \boldsymbol{\zeta}\left(T_{j_{2}}\right)\right)$. Table 14.4 contains the costs of travelling from the initial position of each aircraft $A_{i}$ to any target $T_{j}$, denoted $\mathcal{C}_{i}\left(\boldsymbol{\zeta}_{0}\left(A_{i}\right), \boldsymbol{\zeta}\left(T_{j}\right)\right)$. In this example, costs are assumed to be independent of the aircraft (i.e., all aircraft are assumed to travel at the same speed).

The results obtained using AMPL and CPLEX are presented below. The resulting assignment $\mathcal{A}$ has a cost of 272 seconds, meaning that the mission will 


\begin{tabular}{rr|rrrrrr} 
& & \multicolumn{7}{|c}{$j_{1}$} \\
& & \multicolumn{1}{|c}{1} & \multicolumn{1}{c}{2} & \multicolumn{1}{c}{3} & \multicolumn{1}{c}{4} & \multicolumn{1}{c}{5} \\
\hline & 1 & $0 \mathrm{~s}$ & $112 \mathrm{~s}$ & $73 \mathrm{~s}$ & $42 \mathrm{~s}$ & $82 \mathrm{~s}$ & $72 \mathrm{~s}$ \\
& 2 & $112 \mathrm{~s}$ & $0 \mathrm{~s}$ & $48 \mathrm{~s}$ & $77 \mathrm{~s}$ & $63 \mathrm{~s}$ & $82 \mathrm{~s}$ \\
& 3 & $73 \mathrm{~s}$ & $48 \mathrm{~s}$ & $0 \mathrm{~s}$ & $41 \mathrm{~s}$ & $51 \mathrm{~s}$ & $71 \mathrm{~s}$ \\
& 4 & $42 \mathrm{~s}$ & $77 \mathrm{~s}$ & $41 \mathrm{~s}$ & $0 \mathrm{~s}$ & $70 \mathrm{~s}$ & $41 \mathrm{~s}$ \\
& 5 & $82 \mathrm{~s}$ & $63 \mathrm{~s}$ & $51 \mathrm{~s}$ & $70 \mathrm{~s}$ & $0 \mathrm{~s}$ & $108 \mathrm{~s}$ \\
& 6 & $72 \mathrm{~s}$ & $82 \mathrm{~s}$ & $71 \mathrm{~s}$ & $41 \mathrm{~s}$ & $108 \mathrm{~s}$ & $0 \mathrm{~s}$
\end{tabular}

Table 14.3: Time costs of travelling from $T_{j_{1}}$ to $T_{j_{2}}$.

\begin{tabular}{rr|rrrrrr} 
& & \multicolumn{7}{|c}{$j$} \\
& & \multicolumn{1}{|c}{1} & \multicolumn{1}{c}{2} & 3 & 4 & \multicolumn{1}{c}{5} & 6 \\
\hline \multirow{2}{*}{$i$} & 1 & $90 \mathrm{~s}$ & $108 \mathrm{~s}$ & $99 \mathrm{~s}$ & $63 \mathrm{~s}$ & $95 \mathrm{~s}$ & $28 \mathrm{~s}$ \\
& 2 & $134 \mathrm{~s}$ & $40 \mathrm{~s}$ & $76 \mathrm{~s}$ & $92 \mathrm{~s}$ & $111 \mathrm{~s}$ & $85 \mathrm{~s}$
\end{tabular}

Table 14.4: Time costs of travelling from $A_{i}$ 's initial position to $T_{j}$.

require that time to complete. Table 14.5 shows the route for the aircraft $A_{1}$ and table 14.6 shows the route for aircraft $A_{2}$.

\begin{tabular}{r|cccc} 
& \multicolumn{5}{|c}{$k$} \\
\hline Target & 1 & 2 & 3 & 4 \\
$l$ & $T_{1}$ & $T_{4}$ & $T_{1}$ & $T_{6}$ \\
$c_{i j}^{k l}$ & 1 & 1 & 2 & 1 \\
$\mathrm{~s}$ & $150 \mathrm{~s}$ & $200 \mathrm{~s}$ & $272 \mathrm{~s}$
\end{tabular}

Table 14.5: Route assigned to aircraft $A_{1}$.

\begin{tabular}{r|cccccc} 
& \multicolumn{7}{|c}{$k$} \\
& 1 & 2 & 3 & 4 & 5 & 6 \\
\hline Target & $T_{2}$ & $T_{5}$ & $T_{3}$ & $T_{3}$ & $T_{2}$ & $T_{3}$ \\
$l$ & 1 & 1 & 1 & 2 & 2 & 3 \\
$c_{i j}^{k l}$ & $40 \mathrm{~s}$ & $103 \mathrm{~s}$ & $165 \mathrm{~s}$ & $172 \mathrm{~s}$ & $220 \mathrm{~s}$ & $272 \mathrm{~s}$
\end{tabular}

Table 14.6: Route assigned to aircraft $A_{2}$.

Aircraft $A_{1}$ is assigned all the visits to targets $T_{1}, T_{4}$, and $T_{6}$; whereas aircraft $A_{2}$ is assigned visits to targets $T_{2}, T_{3}, \mathrm{y} T_{5}$. So, there is no target that is visited by both aircraft. This is not a requirement of the formulation, and one target could be visited by two different aircraft if that was the most efficient way to complete the mission.

A schematic map showing the location of all the targets and the movement of each aircraft is provided in figure 14.2. The route in blue corresponds to aircraft $A_{1}$, from its initial location to the last assigned target. The route in red corresponds to the second aircraft. Figure 14.3 shows the targets visited by each aircraft along time.

The duration of the mission is set by the duration of the longest route. In this case, both routes have the same duration. However, note that the route of 

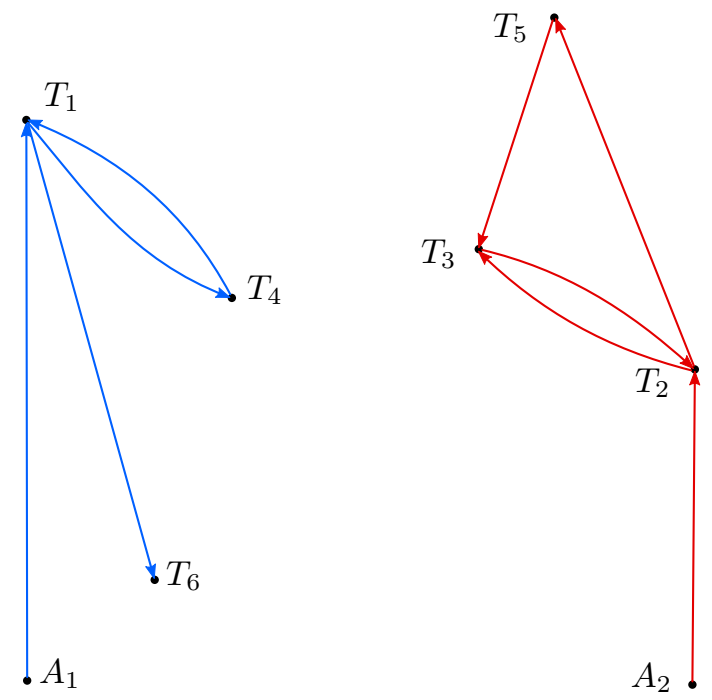

Figure 14.2: Target assignment on a map.

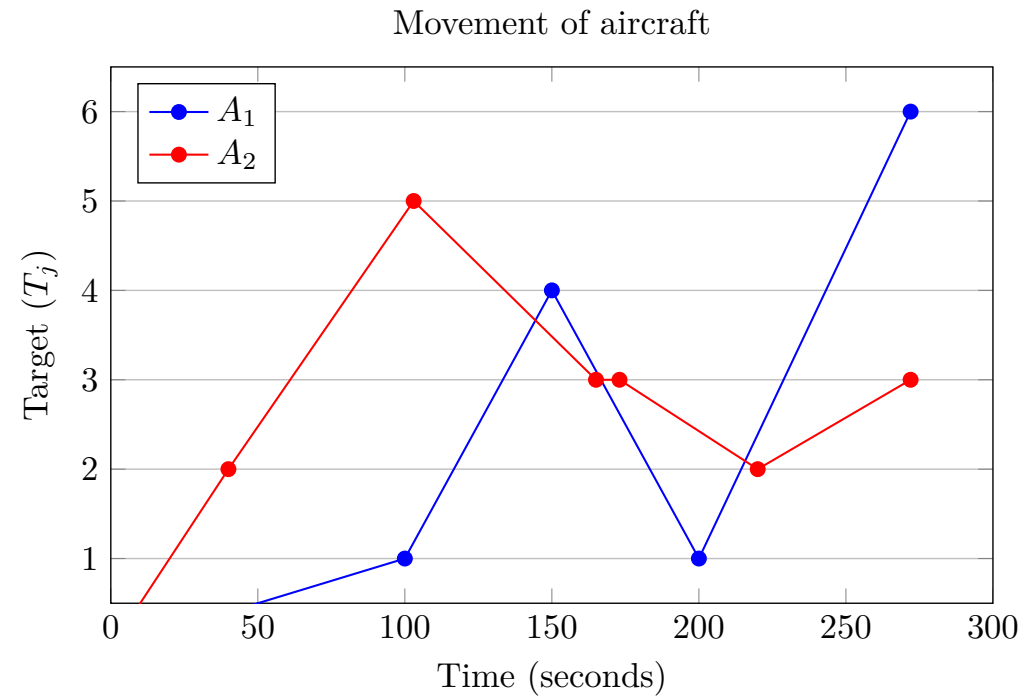

Figure 14.3: Targets visited by aircraft along time. 
aircraft $A_{1}$ is as short as it could be (given the assigned targets), whereas the route of the aircraft $A_{2}$ could be completed faster (in 270 seconds, two seconds less). However, this does not affect the optimization target, defined by the objective function.

The computation of the optimal solution for this example mission took 44 minutes and 40 seconds in a computer with two Intel囚 Xeon® E5-2620 CPUs and 32 gigabytes of RAM. The solver used is heavily multi-threaded, so the total CPU time ${ }^{2}$ was 16 hours, 1 minute, and 37 seconds.

Solving the mission planning problem using the compete formulation presented in section 14.1.4, even for a small number of aircraft and targets, is very expensive computationally. Some improvements may be achieved by replacing the general MILP solver with an algorithm designed specifically to solve the mission planning problem. However, the improvements of such algorithm could be marginal, and the problem would still be impracticable. A more reasonable approach would be to develop methods to obtain sub-optimal assignments. One way of computing sub-optimal assignments is to divide the mission planning problem into a series of smaller sub-problems, as done in [98]. An optimal solution to each of the sub-problems can be obtained much faster and, by combining those solutions, a sub-optimal solution to the whole problem can be constructed.

\subsection{Computing paths in an urban environment}

So far, this chapter hasn't addressed the problem of finding the 'best' path between two locations during the mission. This path is computed by the path planning engine (PPE), using the algorithm described in this section. The 'best' path usually depends on its cost, which can be related to the distance, time, fuel consumption, or risk taken when following the path (or any combination thereof). This section presents a fast algorithm for obtaining approximately shortest paths. In this case, the cost of the path is exclusively related to its distance, so the 'best' path is the shortest one. This doesn't guarantee that it is also the fastest one, but in the absence of more information (e.g., wind maps or aircraft performance), it can be assumed that there is a high correlation between the two. Therefore, the paths computed using this algorithm could be used to estimate the time costs $\mathcal{C}_{i}$ of paths used in the previous section.

A formal description of the problem to be solved in this section is presented below:

The objective of the algorithm is to compute approximately shortest paths in a three-dimensional environment between two points, $u$ and $v$, avoiding the obstacles present in the environment. The input of the algorithm consists of the origin and destination points, $u$ and $v$, and the set of obstacles $O=\left\{o_{i}\right\}$. Obstacles $o_{i}$ in this scenario are vertical polyhedra. Vertical polyhedra are right prisms with their base lying on the ground (i.e. the height of their base is zero), which means that path segments can't be under an obstacle (see figure 14.4).

\footnotetext{
${ }^{2}$ That is, the sum of the computation times for every thread, which may provide a rough estimation of the time that would be necessary in a single-threaded computer. This value is provided by the solver.
} 

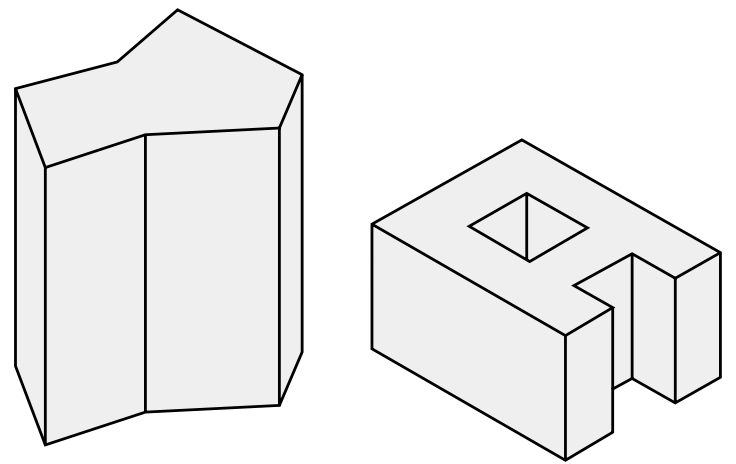

Figure 14.4: Examples of vertical polyhedra.

The output of the algorithm is a path $W$, which is defined as a sequence of points, $W=\left[w_{1}, \ldots, w_{t}\right]$.

It is also assumed that the surface of the obstacles is a collision-free space, that is, the UAs can move closely to such surface. This assumption is rarely correct in real world scenarios, since UAs themselves occupy some volume and their navigation is not perfect. However, this can be easily solved by making the obstacles slightly bigger to include unsafe zones.

\subsubsection{Approximate Shortest Paths}

The method presented here tries to reduce the overall time required for the computation of a collision-free path in an urban environment. Most algorithms try to improve the asymptotic running time, but these theoretical bounds don't necessarily reflect the behaviour on practical situations [120]. Instead, this method improves the time requirements in practical operation, using most usual urban scenarios. This time can be reduced if the information on the type of obstacle geometry is exploited [116]. Furthermore, the problem can be relaxed to make computation even faster. Our proposal relaxes the problem in two ways. First, only a finite number of vertices are allowed to be used as the inner vertices of the resulting path (as opposed to optimal methods, which must consider all points in obstacle edges), making the problem much easier to solve. And second, the number of obstacles taken into account for the path computation is reduced. It will be shown how to reduce the number of obstacles while still guaranteeing that the resulting path is collision-free, using a baseline algorithm similar to [108].

In the next sections, the fundamental parts of the algorithm are detailed. This algorithm is comprised by three smaller algorithms. In section 14.2.2, the ApproximateGrapH algorithm is described, which generates a visibility graph given a set of relevant obstacles. Then, in section 14.2.3, the CORE algorithm is explained. CORE provides the APPROXIMATEGraPh algorithm with the relevant obstacles, and uses the resulting visibility graph to compute a path between two points. Finally, in section 14.2.4 the InCREMENTALPATH algorithm is described, which is built on top of CORE and ensures that the final path does not intersect any obstacle in $O$. 


\subsubsection{Visibility Graph}

The ApproximateGraph algorithm needs to generate a visibility graph given a set of relevant obstacles $Q=\left\{q_{i}\right\}$ (with $Q \subseteq O$ ) and two points, $u^{\prime}$ and $v^{\prime}$, corresponding to the origin and destination. The ApproximateGraph algorithm is an approximation algorithm from [116], but modified for our application. This approximation algorithm is much faster than any of the optimal algorithms found in the literature.

In the scenario described in section 14.2 there is no restriction to the heights of the top faces of the obstacles. However, the approximation algorithm presented here works for a constant number $k$ (with $k \geq 2$ ) of different heights. Therefore, the number of different heights of the obstacles, which can be as many as the number of relevant obstacles, has to be reduced to $k$. This is done by classifying all heights into $k$ different groups, each group corresponding to a height $h_{j}$, with $0=h_{1}<h_{2}<\ldots<h_{k}$. For each obstacle $q_{i}$, its height $H\left(q_{i}\right)$ is assigned to the group with lowest $h_{j}$ that meets $H\left(q_{i}\right) \leq h_{j}$, denoted $H^{\prime}\left(q_{i}\right)$. Once the classification is done, the algorithm will use $H^{\prime}\left(q_{i}\right)$ instead of $H\left(q_{i}\right)$.

The $k$ groups can be fixed, or they can be generated dynamically. The approach used here generates them dynamically, as it leads to more precise results. The maximum height is assigned the value $h_{k}=\max _{i} H\left(q_{i}\right)$; and the rest of the heights, $h_{j}=\frac{j-1}{k-1} h_{k}$. As a result, there are $k$ evenly distributed groups, with a maximum height that matches the height of the tallest relevant obstacle, and a minimum height of zero.

After this process, there are only $k$ levels (i.e., possible values for heights, with level $j$ corresponding to height $h_{j}$ ), so it is now possible to compute the visibility graph using the approximation algorithm. From now on, the algorithm will use the set of relevant obstacles $Q=\left\{q_{i}\right\}$ with heights $H^{\prime}\left(q_{i}\right)$, and two points $u^{\prime}$ and $v^{\prime}$. The output is a three-dimensional visibility graph which includes $u^{\prime}$, $v^{\prime}$, all the vertices of the obstacles in $Q$, and additional vertices on some edges of such obstacles.

The APPRoximateGrapH algorithm relies on the generation of $k 2 \mathrm{D}$ visibility graphs, $G_{1}, \ldots, G_{k}$, corresponding to the $k$ levels. The $2 \mathrm{D}$ visibility graphs generated include the vertical projection of all points at and underneath the current level, for a total of $n$ vertices in any level. Note that only upwards projections are necessary, as lower levels already contain the projections of points above them (obstacles lie on the base level $h_{1}$ ). Figure 14.5 depicts the resulting $2 \mathrm{D}$ visibility graph in an environment with three levels $(k=3)$. An improved version of the algorithm proposed in [103] is defined to solve the $2 \mathrm{D}$ visibility graph problem in $O\left(n^{2}\right)$ time. This algorithm stores, for each pair of vertices that are not connected by the graph, the face that occludes visibility, so that this information can be later retrieved in $O(1)$ time. This modification does not increase the asymptotic time required by this part of our algorithm.

The set of $G_{1}, \ldots, G_{k}$ shows $k 2 \mathrm{D}$ visibility graphs in a $3 \mathrm{D}$ environment at various altitude levels, as depicted in figure 14.5. However, this visibility graph set lacks any edges connecting different levels. The next step of the algorithm is to generate a three-dimensional visibility graph $G$ that connects different levels. The process described here is based on the approach described in [116].

Let $V_{j}$ be the set of vertices of $G_{j}$, with cardinality $\left|V_{j}\right|=n$, and let $r_{k}$ be 


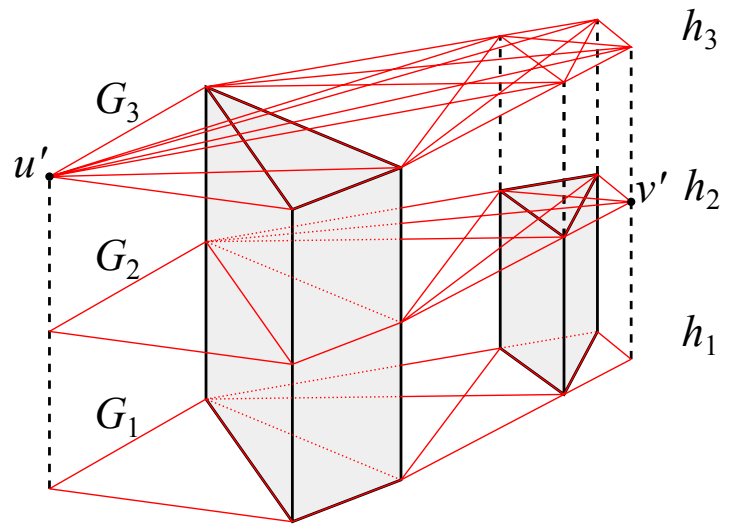

Figure 14.5: Two-dimensional visibility graph for three levels $\left(h_{3}, h_{2}\right.$ and $\left.h_{1}\right)$.

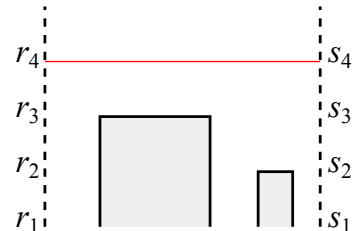

(a)

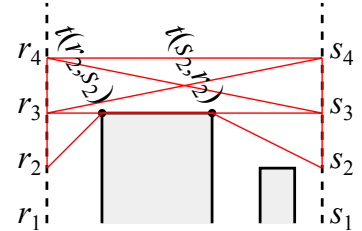

(c)

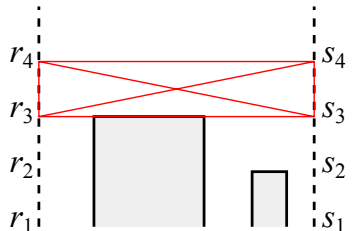

(b)

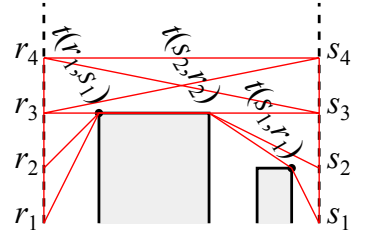

(d)

Figure 14.6: Connecting vertices $r_{4}, s_{4}$, and their vertical projections in different levels. Note $t\left(r_{2}, s_{2}\right)=t\left(r_{1}, s_{1}\right)$.

any vertex of $V_{k}$. As all vertices are projected to all levels, the projection of $r_{k}$ at level $j$, denoted $r_{j}$, is in $V_{j}$. The pair connecting process takes two vertices $r_{k}, s_{k} \in V_{k}$, and adds the appropriate connections between $r_{j}$ and $s_{j^{\prime}}$ for any $j$ and $j^{\prime}\left(j, j^{\prime} \leq k\right)$. This process requires $O\left(k^{2}\right)$ time. In order to connect all vertices in all levels, the pair connecting process is repeated for every $r_{k}, s_{k} \in V_{k}$ such that $r_{k} \neq s_{k}$, which requires $O\left(k^{2} n^{2}\right)$ time in total.

Figure 14.6 shows a vertical cut of the space and obstacles between two points $r_{4}$ and $s_{4}$, with the edges created at different stages of the pair connecting process. While this figure shows a $2 \mathrm{D}$ cut of the environment, in figure 14.7 similar results are shown in a $3 \mathrm{D}$ environment.

As stated before, the pair connecting process starts with two vertices $r_{k}, s_{k} \in$ $V_{k}$. These vertices are connected in $G_{k}$ ( $G_{k}$ is always a complete graph) so an edge from $r_{k}$ to $s_{k}$ is added to the visibility graph $G$ (see figure 14.6a). The process then continues with the remaining $k-1$ levels, descending one level at 


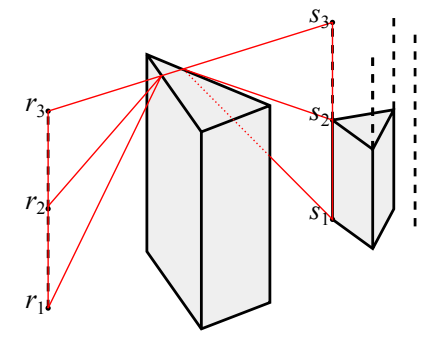

(a)

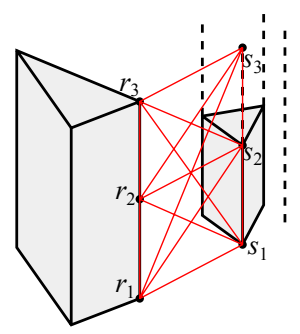

(b)

Figure 14.7: Edges added by the pair connecting process for different pairs of vertices $r_{k}$ and $s_{k}$.

a time, on a vertical plane containing the segment $\overline{r_{k} s_{k}}$. At each level $j$, the process performs as follows:

- If $r_{j}$ and $s_{j}$ are connected in $G_{j}$, edges from $r_{j}$ to $s_{j^{\prime}}$ and edges from $s_{j}$ to $r_{j^{\prime}}$ are added to $G$, for all $j^{\prime} \geq j$. In other words, $r_{j}$ is connected to all projections of $s_{j}$ at or above current level, and vice versa (see figure 14.6b).

- If $r_{j}$ and $s_{j}$ are not connected in $G_{j}$, but $r_{j+1}$ and $s_{j+1}$ were connected in $G_{j+1}$, the process computes a path from $r_{j}$ to $s_{j}$ above the obstacles in between, i.e., a sector of the path would be at level $j+1$.

For convenience, the first intersection from $r_{j}$ to $s_{j}$ is defined as the intersection nearest to $r_{j}$ of the segment $\overline{r_{j} s_{j}}$ with an obstacle in $Q$. This intersection can be obtained in $O(1)$ time as the intersecting face has been precomputed. The first intersection from $r_{j}$ to $s_{j}$ can be projected upwards to $H_{t o p}^{\prime}\left(q_{i}\right)$ (where $q_{i}$ is the intersecting obstacle) to obtain the horizon from $r_{j}$ to $s_{j}$, denoted $t\left(r_{j}, s_{j}\right)$.

The computed path that goes from $r_{j}$ to $s_{j}$ is formed by three edges: one connecting $r_{j}$ with $t\left(r_{j}, s_{j}\right)$, one horizontal edge connecting $t\left(r_{j}, s_{j}\right)$ with $t\left(s_{j}, r_{j}\right)$, and another one connecting $t\left(s_{j}, r_{j}\right)$ with $s_{j}$ (see figure 14.6c). These edges are added to $G$.

- If $r_{j}$ and $s_{j}$ are not connected in $G_{j}$, nor $r_{j+1}$ and $s_{j+1}$ were connected in $G_{j+1}$, then the process computes a path from $r_{j}$ to $t\left(r_{j+1}, s_{j+1}\right)$ and a path from $s_{j}$ to $t\left(s_{j+1}, r_{j+1}\right)$. The computation process of one of these paths is analogous to the other, so only the former is detailed here.

If $t\left(r_{j}, s_{j}\right)=t\left(r_{j+1}, s_{j+1}\right)$, said path consists of one edge connecting $r_{j}$ with $t\left(r_{j}, s_{j}\right)$. Otherwise, the path consists of two edges: one connecting $r_{j}$ with $t\left(r_{j}, s_{j}\right)$, and one connecting $t\left(r_{j}, s_{j}\right)$ with $t\left(r_{j+1}, s_{j+1}\right)$ (see figure 14.6d). In any case, the edges comprising the computed path are added to $G$.

Some examples of edges added by the pair connecting process for different pairs of vertices are shown in figure 14.7. This process is repeated for every $r_{k}, s_{k} \in V_{k}$ such that $r_{k} \neq s_{k}$. This results in a 3D visibility graph that includes:

1. all the vertices of the obstacles, projected at $k$ levels (with height $h_{j}$ ); 
2. additional vertices for paths above obstacles;

3. edges between vertices at the same or different heights.

Overall, the cost of computing a $3 \mathrm{D}$ visibility graph using this method is $O\left(k^{2} n^{2}\right)$ time. This is equivalent to $O\left(n^{2}\right)$ time as $k$ is a constant.

\subsubsection{Filtering obstacles}

In section 14.2 .2 , an algorithm that generates a visibility graph given a set of relevant obstacles and two points was described. In this section the CORE algorithm is described, which generates the set $Q$ of relevant obstacles, and computes the shortest path between $u^{\prime}$ and $v^{\prime}$ given the visibility graph $G$ provided by the ApproximateGraph algorithm. Pseudo-code for Core is shown in algorithm 1.

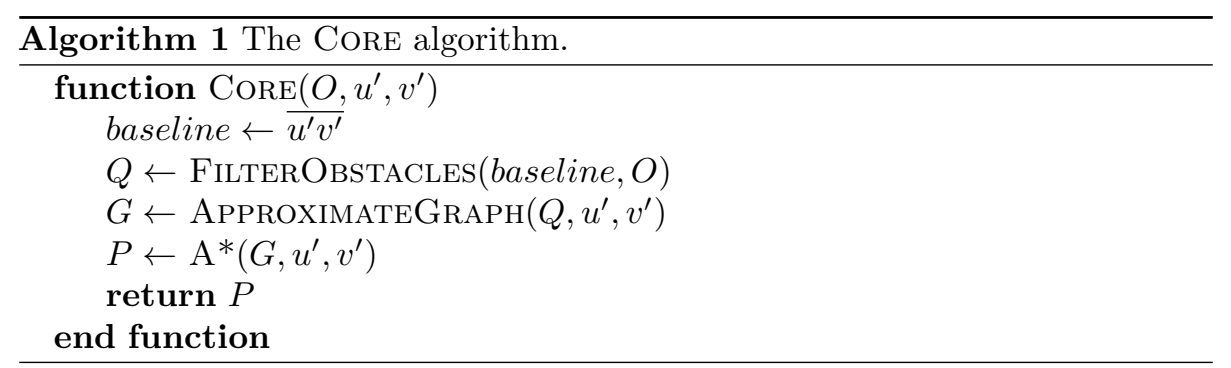

The input of the CORE algorithm is the set of obstacles $O$, and points $u^{\prime}$ and $v^{\prime}$. First, the algorithm computes the baseline, which is defined as the segment that goes from $u^{\prime}$ to $v^{\prime}$. The set $Q$ of relevant obstacles is the set of obstacles from $O$ that are intersected by the baseline. This set is computed by the FilterObstacles function outlined in algorithm 2, where Intersects determines whether or not the baseline intersects a given obstacle. Next, a visibility graph is generated for the relevant obstacles (using the APPROXIMATEGRAPH described in 14.2.2) and a path from $u^{\prime}$ to $v^{\prime}$ (specified as a sequence of points $\left.P=\left[p_{1}, \ldots, p_{s}\right]\right)$ is obtained using the $\mathrm{A}^{*}$ algorithm. The resulting path always contains $u^{\prime}$ and $v^{\prime}$ (since they are the origin and destination points), so $p_{1}=u^{\prime}$ and $p_{s}=v^{\prime}$.

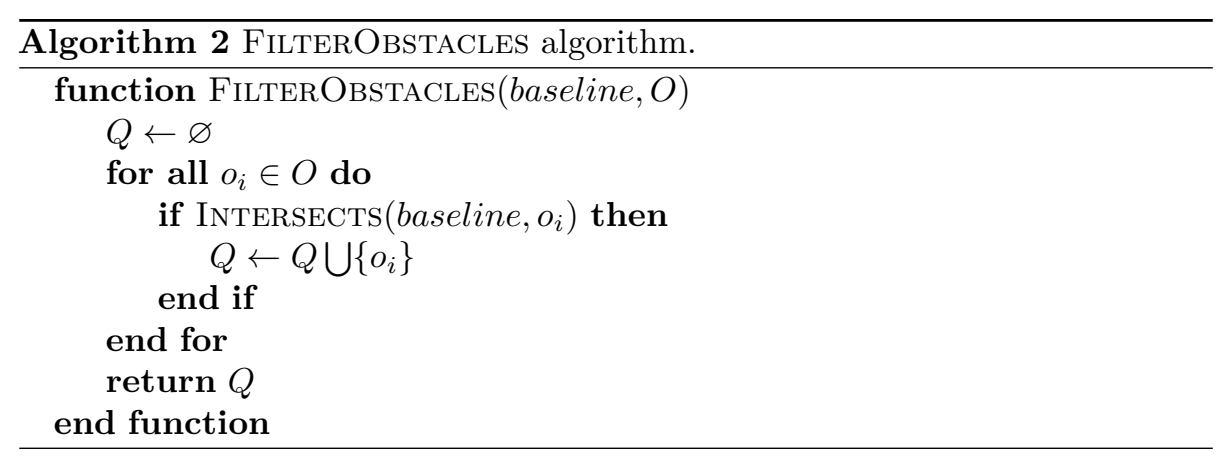

Note that, although the path $P$ generated by CoRE does not intersect any of the relevant obstacles, it may intersect one of the obstacles of $O$ that isn't in $Q$. 


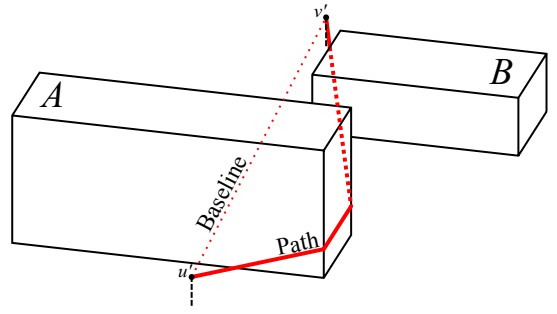

(a) Path with collision.

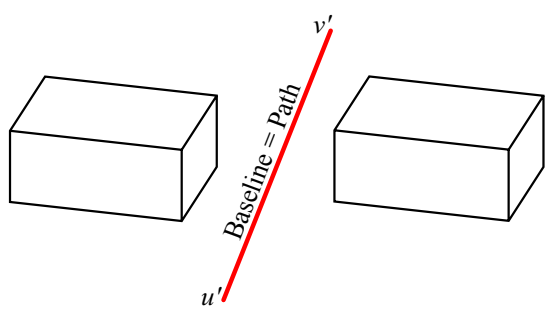

(b) Collision-free path.

Figure 14.8: Different paths generated by CoRE.

An example of this situation is shown in figure 14.8a. In the figure, the baseline only intersects obstacle $A$. CoRE generates a path that avoids $A$, but this path intersects $B$, because it was initially discarded. However, when the baseline does not intersect any obstacle (there are no relevant obstacles), the path generated by CORE is exactly a straight path from $u^{\prime}$ to $v^{\prime}$, which is collision-free (see figure $14.8 \mathrm{~b})$.

\subsubsection{Building a path incrementally}

To overcome the situation where the path provided by CORE intersects an obstacle, the final path is built incrementally, making successive calls to CORE until a complete obstacle-free path is obtained. The IncrementalPath method that builds a collision-free path is shown in algorithm 3 . It is an adapted version of the BLOVL algorithm described in [108].

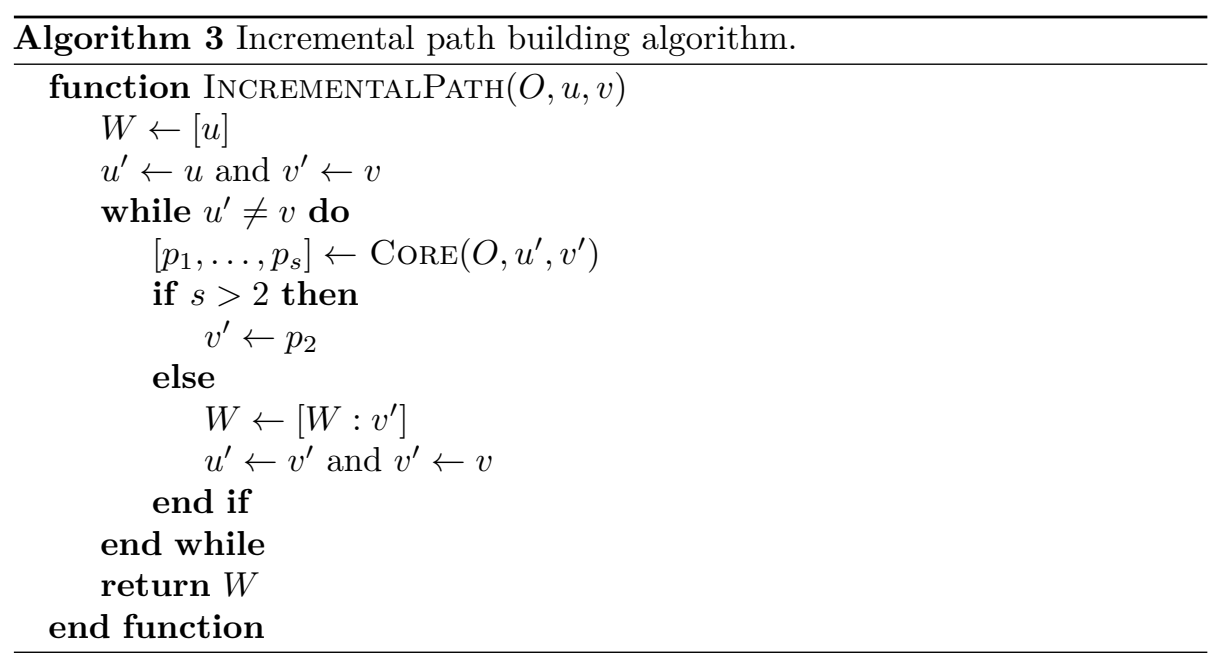

Consider the scenario with a set of obstacles $O$, and points $u$ and $v$. The INCREMENTALPATH algorithm incrementally builds a working path $W$, which is always obstacle-free. Initially, the working path contains only the initial point, $u$. The algorithm will append new points to the end of $W$ until it reaches $v$.

The InCREMENTALPATH algorithm will use intermediate points $u^{\prime}$ and $v^{\prime}$ to compute partial paths. Initially, these intermediate points $u^{\prime}$ and $v^{\prime}$ take the 


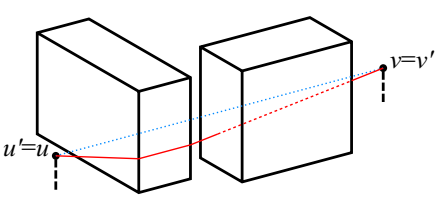

(a) First iteration

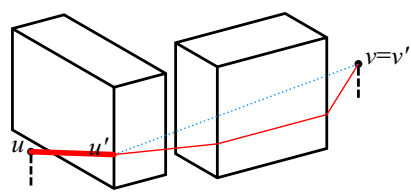

(c) Third iteration

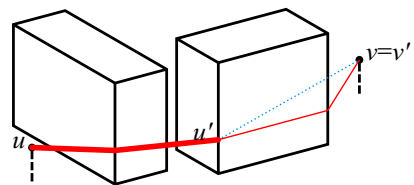

(e) Fifth iteration

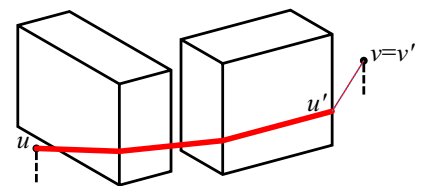

(g) Seventh iteration

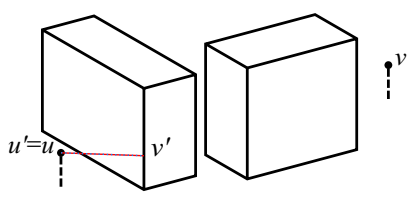

(b) Second iteration

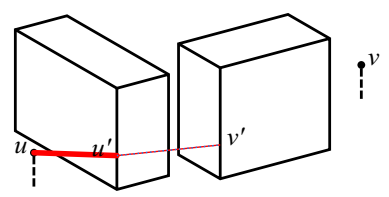

(d) Fourth iteration

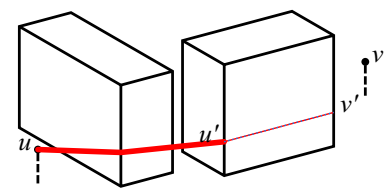

(f) Sixth iteration

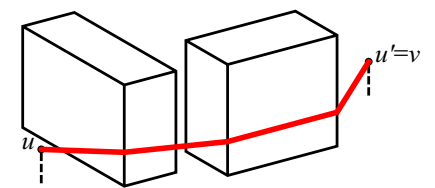

(h) Eighth iteration

Figure 14.9: Example execution of the InCREMENTALPATH algorithm.

values of $u$ and $v$, respectively. CORE is called iteratively to compute the partial path from $u^{\prime}$ to $v^{\prime}$. If the CORE algorithm identified some obstacles in the way, then the resulting path $\left[p_{1}, p_{2}, \ldots, p_{s}\right]$ is a sequence containing more than two points. However, although this partial path avoids all relevant obstacles, CORE does not guarantee that it avoids all the obstacles in $O$. In particular, it is not guaranteed that the first segment of the path avoids all the obstacles. Therefore, the points conforming that first segment, $p_{1}$ and $p_{2}$, are used as intermediate points, $u^{\prime}$ to $v^{\prime}$, for the next iteration (note $p_{1}=u^{\prime}$, so in this case $u^{\prime}$ does not change). On the other hand, if the CoRE algorithm didn't identify any obstacles in the way, then the resulting path is a sequence containing exactly two points (the path is $\left[u^{\prime}, v^{\prime}\right]$ ). In this case, the partial path is collision-free: $v^{\prime}$ can be appended at the end of the working path $W\left(\left[W: v^{\prime}\right]\right.$ denotes a list with element $v^{\prime}$ appended at the end); and the intermediate points in the next iteration will be $v^{\prime}$ and $v$ in order to compute the remaining path to $v$. Once the working path reaches $v$ (that is, when $u^{\prime}=v=w_{t}$ ), a path from $u$ to $v$ has been found. This path is guaranteed to be collision-free.

An example of how the InCREmentalPath algorithm computes a path is shown in figure 14.9. A dotted line is used for the baseline, a narrow line is used for the partial paths and a thicker line is used for the working path. In the first call to CORE (a), only one of the obstacles is intersected by the baseline 
and the partial obtained path intersects another obstacle. In the second call (b), the algorithm checks that the first segment of the previous path is obstaclefree. As it is the case, adds $v^{\prime}$ to the working path. In the third call (c), the baseline intersects both obstacles, and a different path is returned. This time the returned path is obstacle-free. Still, the algorithm has to call CorE a fourth time (d) to check the first segment of the path before adding $v^{\prime}$ to the working path. Calls to CoRE continue until the working path reaches $v$.

\subsubsection{Results}

To assess the performance of the proposed algorithm, a Monte Carlo method is used, which compares the paths obtained by four different algorithms, including the one just described. Several Monte Carlo simulations have been run, with different numbers of obstacles. Each simulation comprises 500 different randomly generated scenarios, and every scenario is solved using the four algorithms chosen for the comparison. Randomly generated scenarios consist of a $1 \mathrm{~km}^{2}$ surface with the corresponding number of obstacles on it, and the two ends of the path, $u$ and $v$. Obstacles have a rectangular base with random width and depth (between 5 to $40 \mathrm{~m}$ ), and random height (between 4 and $40 \mathrm{~m}$ ).

These simulations compare the following four non-exact algorithms:

1. Sub-sampling algorithm (SuBS). The first of these algorithms divides obstacle edges in small segments (not longer than 1 meter), as proposed in [117] or [118], increasing the number of vertices significantly. Then, the algorithm builds the shortest path in which all inner vertices must be vertices of the obstacles. In this implementation the visibility graph is not completely built. Instead, visibility between vertices is computed as the A* algorithm requests such visibility information, and not before. This algorithm requires $O\left(n^{3}\right)$ time to complete in the worst case.

2. Approximation algorithm (APPR.). The algorithm developed by Gewali et al. [116] is the second algorithm used in the simulations. This algorithm requires $O\left(k^{2} n^{2}\right)$ time to complete. As $k$ is a constant (in these simulations $k=10$ was used), that cost is equivalent to $O\left(n^{2}\right)$ time in the worst case.

3. Visibility line based algorithm in 3D (VLB3D). The algorithm proposed in [123] is the third algorithm used in the simulations. This algorithm generates several planes around the segment that connects $u$ and $v$, computes the intersection of each plane with the obstacles and solves a 2ESP problem for each plane. The shortest path among the planes is chosen as the approximate 3ESP. In this implementation, 6 different planes are generated (with increments of $\frac{\pi}{4}$ degrees). This algorithm computes a path in $O\left(n^{2}\right)$ time in the worst case.

4. Approximation algorithm with visibility line algorithm (APVL). The last evaluated algorithm is the one proposed in this chapter. This implementation requires $O\left(k^{2} n^{3}\right)$ time to compute a path in the worst case. Again, as $k$ is a constant ( $k=10$ was also used for this algorithm), this is equivalent to $O\left(n^{3}\right)$ time. 


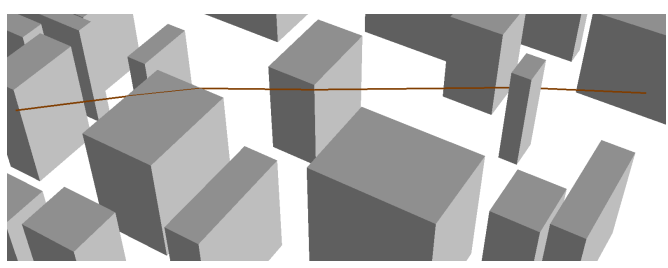

(a) Path generated by SuBS.

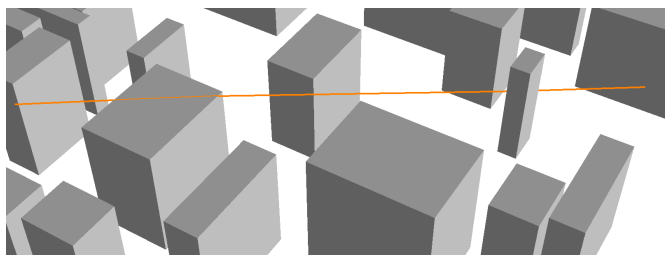

(c) Path generated by VLB3D.

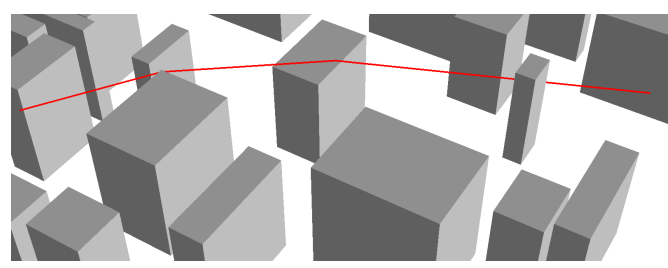

(b) Path generated by APPR.

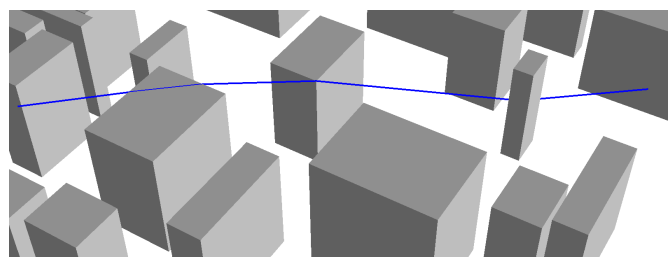

(d) Path generated by APVL.

Figure 14.10: Example of different paths generated by each algorithm for the same scenario.

These four algorithms do not return, in general, the same path for a given scenario. Figure 14.10 shows an scenario where each algorithm provides a different path. The sub-sampling algorithm is expected to provide the shortest paths, with results quite similar to the optimal solution (although it does so with extremely high time cost). Therefore, those paths are used as a reference to measure the quality of the paths generated by the other algorithms. Once the four paths for a given scenario are computed, the length ratio of each path (i.e., the length of such path divided by the length of the reference path) is measured. Also, the time spent on the computation of each of the paths is measured. Simulations were run on an Intel Xeon 2.0GHz CPU with 8GB of RAM under Linux 3.13. Results of the simulation are presented in tables 14.7 to 14.10 . These tables include the number of obstacles of each simulation, and the mean and 95th percentile of the two measured values (length ratio and running time).

Results for the SUBS algorithm in the comparative analysis are shown in table 14.7. As the paths provided by this algorithm are used as the reference, the length ratio measures are omitted in this table. These results show that, in practice, the mean time required to compute a path is approximately proportional to the cubed number of obstacles. Computation times range from the hundreds of milliseconds to several minutes, which make this algorithm unsuitable for real-time path finding.

Table 14.8 shows the results of the APPR. algorithm. This algorithm guarantees that paths are not more than $8 \%$ longer than the optimum path when some conditions are met. In practice, the simulation reveals that they are rarely more than $1 \%$ longer than the reference length. It can also be observed that the running times of this algorithm are also approximately proportional to the squared number of obstacles, which matches the worst-case time complexity. In fact, the mean running time and the 95th percentile are very close in most cases. When comparing the running times of this algorithm with SuBS, it can be seen that for a small number of obstacles, SuBS requires less time. But, as 


\begin{tabular}{lrr}
\hline \multirow{2}{*}{ \# obst. } & \multicolumn{2}{c}{ Running time (ms) } \\
\cline { 2 - 3 } & Mean & $95 \%$ ile. \\
\hline \hline 20 & 258 & 938 \\
40 & 1,613 & 5,837 \\
60 & 9,232 & 42,952 \\
80 & 15,964 & 88,631 \\
100 & 36,993 & 160,749 \\
120 & 75,793 & 383,537 \\
140 & 99,320 & 546,701 \\
160 & 160,847 & 930,496 \\
180 & 291,233 & $1,399,430$ \\
200 & 357,048 & $1,769,160$ \\
\hline
\end{tabular}

Table 14.7: SuBS algorithm results

the number of obstacles grow, this algorithm starts performing better. However, this algorithm also breaks the barrier of one minute for some of the scenarios in the simulation, which makes this algorithm still not fast enough for real time path finding. Another problem we found with this algorithm is its memory requirements - also in $O\left(n^{2}\right)$ - which made it impossible to provide results for a number of obstacles greater than 160 .

\begin{tabular}{lrrrr}
\hline \multirow{2}{*}{ \# obst. } & \multicolumn{2}{c}{ Length ratio } & \multicolumn{2}{c}{ Running time $(\mathrm{ms})$} \\
\cline { 2 - 5 } & Mean & 95\%ile. & Mean & $95 \%$ ile. \\
\hline \hline 20 & 1.00010 & 1.00056 & 746.8 & 785.7 \\
40 & 1.00036 & 1.00135 & $3,186.0$ & $3,340.8$ \\
60 & 1.00035 & 1.00180 & $7,094.0$ & $7,314.3$ \\
80 & 1.00055 & 1.00239 & $13,308.7$ & $13,813.3$ \\
100 & 1.00079 & 1.00302 & $22,080.3$ & $23,341.7$ \\
120 & 1.00126 & 1.00513 & $23,341.7$ & $33,258.0$ \\
140 & 1.00083 & 1.00328 & $45,238.2$ & $48,322.8$ \\
160 & 1.00113 & 1.00397 & $58,765.7$ & $66,702.0$ \\
\hline
\end{tabular}

Table 14.8: ApPR. algorithm results

The results in table 14.9 for the VLB3D algorithm show promising results. The paths generated by this algorithm are less than $0.5 \%$ longer than the reference path. Running times are in this case also approximately proportional to the squared number of obstacles, which again matches the worst-case time complexity. For the numbers of obstacles used in the simulations, running times range from tens of milliseconds to a few seconds. These results indicate that this algorithm is a good approach for real time path finding when the computation time is allowed to be a few seconds. 


\begin{tabular}{lrrrr}
\hline \multirow{2}{*}{ \# obst. } & \multicolumn{2}{c}{ Length ratio } & \multicolumn{2}{c}{ Running time $(\mathrm{ms})$} \\
\cline { 2 - 5 } & Mean & $95 \%$ ile. & Mean & $95 \%$ ile. \\
\hline \hline 20 & 1.0 & 1.0 & 14.54 & 38.96 \\
40 & 1.00003 & 1.0 & 55.74 & 158.64 \\
60 & 1.00005 & 1.00019 & 125.58 & 309.60 \\
80 & 0.99997 & 1.00030 & 211.87 & 575.37 \\
100 & 1.00015 & 1.00067 & 316.05 & 930.52 \\
120 & 1.00018 & 1.00113 & 469.42 & $1,408.91$ \\
140 & 1.00023 & 1.00095 & 596.74 & $1,801.89$ \\
160 & 1.00118 & 1.00270 & 804.52 & $2,359.95$ \\
180 & 1.00043 & 1.00222 & $1,061.54$ & $3,370.26$ \\
200 & 1.00367 & 1.00383 & $1,291.98$ & $3,968.48$ \\
\hline
\end{tabular}

Table 14.9: VLB3D algorithm results

Finally, the results of the APVL algorithm described in this chapter are shown in table 14.10. The differences in length between the paths generated by this algorithm and the paths generated by the reference algorithm are less than $0.1 \%$ in most cases. But the main difference of these results when compared to the results of any other algorithm is in the running time. Although the worstcase time complexity of this algorithm is $O\left(n^{3}\right)$, simulation results show the running time does not grow as fast as $O\left(n^{3}\right)$. Paths can be generated in the order of milliseconds, which allows to compute hundreds of paths per second, making this algorithm really suitable for real time path finding.

\begin{tabular}{lrrrr}
\hline \multirow{2}{*}{ \# obst. } & \multicolumn{2}{c}{ Length ratio } & \multicolumn{2}{c}{ Running time $(\mathrm{ms})$} \\
\cline { 2 - 5 } & Mean & $95 \%$ ile. & Mean & $95 \%$ ile. \\
\hline \hline 20 & 1.00002 & 1.00007 & 0.364 & 1.831 \\
40 & 1.00007 & 1.00016 & 0.838 & 3.942 \\
60 & 1.00007 & 1.00031 & 1.575 & 5.884 \\
80 & 1.00014 & 1.00037 & 2.401 & 11.074 \\
100 & 1.00012 & 1.00038 & 2.945 & 12.074 \\
120 & 1.00020 & 1.00060 & 5.514 & 22.336 \\
140 & 1.00018 & 1.00066 & 6.160 & 24.232 \\
160 & 1.00021 & 1.00081 & 8.374 & 37.552 \\
180 & 1.00017 & 1.00085 & 9.203 & 39.399 \\
200 & 1.00022 & 1.00122 & 11.209 & 50.641 \\
\hline
\end{tabular}

Table 14.10: APVL algorithm results

The experimental results show that there can be huge differences in the computation times by the different algorithms considered. These differences cannot 

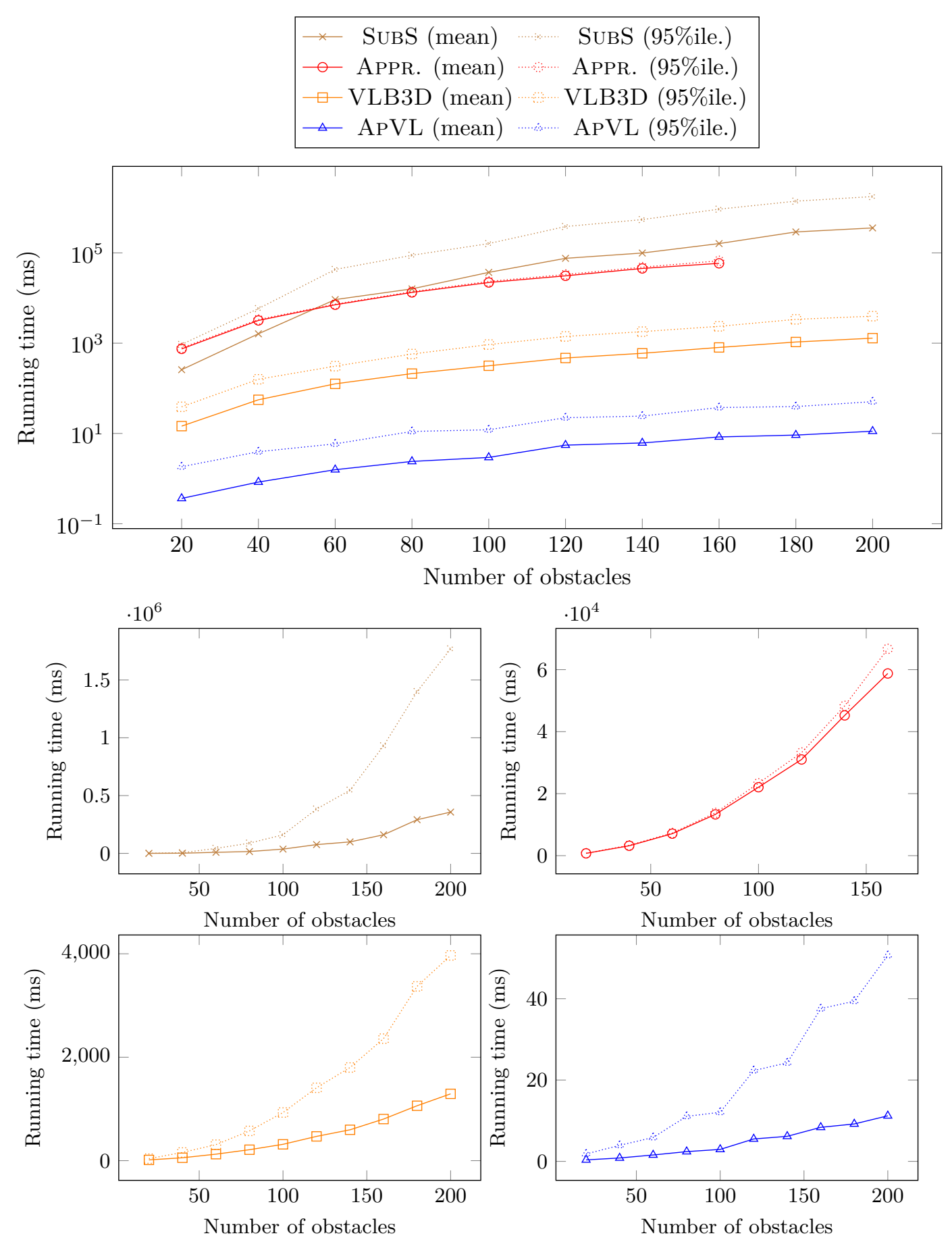

Figure 14.11: Mean and 95th percentile of running time of algorithms versus number of obstacles. 


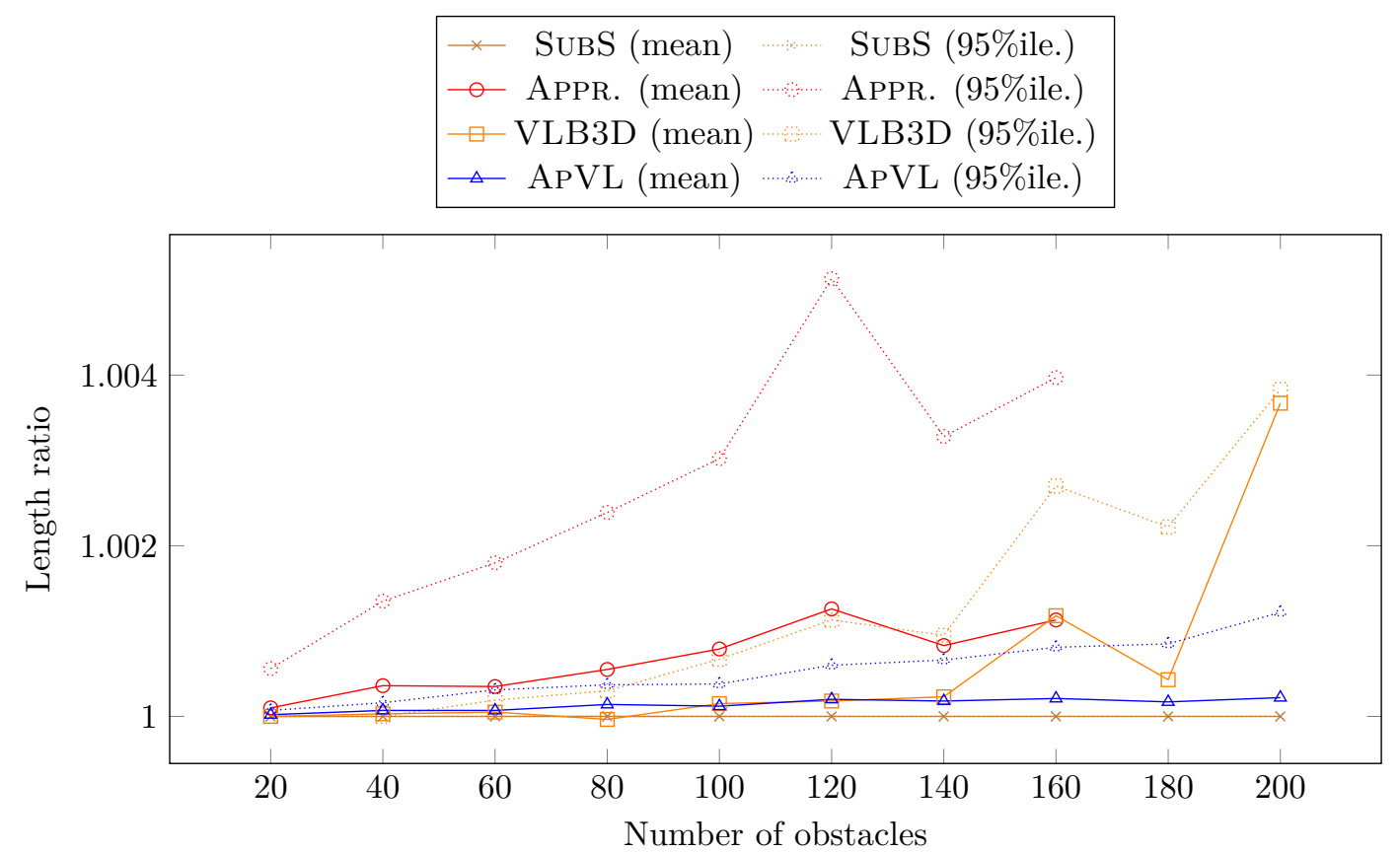

Figure 14.12: Mean length ratio of algorithms' paths versus number of obstacles.

be predicted using the asymptotic worst-case time complexity of the algorithms, which is the most common metric in algorithm analysis. Average-case time complexity can be a more accurate metric. Here, we presented empirical metrics to assess the performance of the four algorithms. While empirical metrics can be misleading in some circumstances, their performance in a significant amount of differently sized scenarios have been tested, providing a reasonable prediction of how the algorithms will perform in these scenarios. Figure 14.11 shows this difference graphically. In the first plot of this figure, all running times (mean times and percentiles) are displayed together in a logarithmic scale. Running time of each algorithm is displayed separately in the plots below, which use a linear scale. In these plots we can see how the running time grows with the number of obstacles.

Differences in path lengths among the compared algorithms are minor. Figure 14.12 shows the average relative difference among path lengths. Average differences of the APVL algorithm with respect to the reference path are less than $0.1 \%$, although the 95 th percentile shows differences in path length up to $0.2 \%$.

\subsection{Final words}

This chapter has provided some insight into the problem of planning a mission. A precise description of the particular mission planning problem solved here has been provided, which is a generalization of the VRPTW. A simple 
MILP formulation to this problem has also been provided, which relies on precomputed estimated travelling times between important locations during the mission. This required estimations reveal the importance of a fast path finding algorithm, which has also been developed in this chapter.

An algorithm designed to find near-optimal paths in urban environments has been presented, which can be used for real-time applications. The algorithm uses several techniques to reduce the computational costs of the paths, some of which were introduced in $[103,108,116]$. In combination, these techniques provide important gains in computational cost. Despite not being efficient from a worst-case time complexity viewpoint (as there are other approximate algorithms with lower computational cost for worst-case time complexity [116, 118, 123]), empirical metrics show that in scenarios that recreate the conditions of an urban environment, this algorithm is much faster than the alternatives, with no significant differences in the quality of the paths provided. 



\section{Part IV}

\section{Conclusions and Bibliography}





\section{Chapter 15}

\section{Conclusions}

This thesis has explored some of the applications of formal languages to air traffic management (ATM) and unmanned aircraft systems (UASs). Several languages have been defined that, in combination, can describe the movement of aircraft in any stage of the flight, and with an arbitrary level of detail. These languages can be better exploited if automated tools utilize them as their primary input and output. These automated tools include - but are not limited to - trajectory predictors and mission planners. A modular trajectory predictor that relies heavily on the defined languages has been designed. The modularity of this trajectory predictor can provide more flexibility than previously existing trajectory predictors, as it is not tied to a particular model or type of aircraft, while it can still provide trajectories with the same quality.

Additionally, some insights on the use of the defined formal languages in mission planning have been provided. The analysis of the mission planning problem has revealed that an effective and efficient way of computing paths is critical to the problem. Thus, an algorithm has been developed to minimize the time invested in path finding, while still obtaining near-optimal paths.

The remainder of this chapter contains an overview of the challenges and solutions developed in this thesis, a listing of the main contributions, and a description of the lines of work that remain open for further development.

\subsection{Overview}

Among all the possible formal languages that could be applied to management of manned and unmanned aircraft, this thesis focuses on those languages that can be used to describe aircraft trajectories. Much of the effort in this thesis was devoted to define a series of formal languages that can fulfil this purpose. Most of these languages have been organized in a hierarchy reflecting the relationships between them.

Previously, Boeing Research 83 Technology Europe (BR\&TE) had already developed the aircraft intent description language (AIDL), a formal language derived from the equations of motion of fixed-wing aircraft that can be used to describe an aircraft's trajectory completely and univocally. This makes it a 
mathematically sound language, ideal to set the foundations of the hierarchy of languages. Unfortunately, this AIDL - denoted fixed-wing AIDL (FW-AIDL) in this thesis for clarity - is tightly bonded with the equations of motion of a fixed-wing aircraft and cannot be used to describe trajectories of other types of aircraft, such as quadrotors.

As the first step towards the building of the language hierarchy adequate for quadrotors, it was necessary to derive a formal language as complete and powerful as the FW-AIDL, but oriented to this kind of aircraft. From the equations of motion of a quadrotor, the same analysis used for the FW-AIDL was followed. The analysis concluded that the equations of motion with three mechanical degrees of freedom (DoFs) can be used to derive a language analogous to the FW-AIDL, denoted quadrotor AIDL (QR-AIDL). This language can be used to unambiguously describe the trajectory of quadrotor aircraft. In an aircraft intent expressed in QR-AIDL, four execution threads contain a sequence of AIDL instructions each. Every AIDL instruction represents a constraint closing one of the four mathematical DoFs (which don't match the mechanical DoFs). The QR-AIDL also contains a series of rules guaranteeing that the four mathematical DoFs of the equations of motion are closed during the whole trajectory.

The FW-AIDL and QR-AIDL provide an unambiguous description of how an aircraft is operated by the pilot, operator, or flight management system (FMS). In other words, given an aircraft intent expressed in either variant of the AIDL, the capabilities of the aircraft - i.e., the aircraft performance model-, and the environmental conditions, the resulting trajectory is univocal. This is extremely important in functions such as final stages of trajectory negotiation but, in other contexts, that much detail may not be necessary, or not even desirable. With these conclusions, the concept of a hierarchy of languages begins to take shape. Other languages were built on top of the AIDL. Those languages can describe trajectories where some information on the flight is not provided. These languages provide more flexibility while maintaining the mathematical soundness of the AIDL.

The idea of a hierarchy of languages with the AIDL at its foundations existed prior to this thesis. Two languages, in addition to the AIDL, composed this hierarchy: the intent composite description language (ICDL) and the flight intent description language (FIDL). Contributions have been made to these languages (in collaboration with BR\&TE) to make them reach the mature state they are today.

A trajectory described using the AIDL must be unambiguous, and thus the rules of this language may be too strict for some applications. For example, it was found that, in trajectory prediction, the horizontal and vertical profiles were usually built separately, which the AIDL doesn't allow. The ICDL addresses the needs in some of these situations. This language adds a layer of abstraction on top of the AIDL, allowing to group a small combination of AIDL instructions into a more conceptual manoeuvre denoted 'elementary composite'. Examples of manoeuvres that can be defined using elementary composites are a 'level flight', a 'Mach-CAS descent' (affecting the vertical profile) or 'fly direct to a point' (affecting the horizontal profile). Using the rules of the ICDL, elementary composites can be combined to form more complex composites. This formal language also provides new semantics, such as conditional branches or loops, 


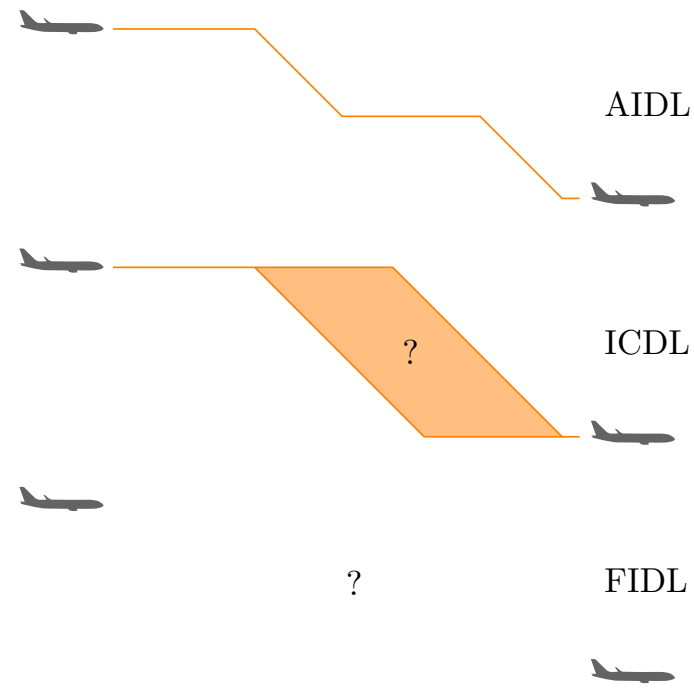

Figure 15.1: Representation of abstraction capabilities in AIDL, ICDL, and FIDL.

allowing non-linear execution of the intent. A trajectory described using the ICDL can leave some magnitudes unspecified, so they can later be set in a optimization process.

Despite the increased flexibility provided by the ICDL, some situations require an even higher level of abstraction in trajectory description. Flight plans, for example, can be used to describe trajectories with a level of abstraction that cannot be achieved by neither the AIDL or the ICDL. Built on top of the ICDL, the FIDL provides that additional layer of abstraction. In FIDL, it is possible not only to describe the desired behaviour of the aircraft, but it is also possible to define what must be achieved, in the form of constraints and objectives. Constraints represent restrictions on the aircraft's trajectory, whereas objectives represent optimization goals. The behaviour of the aircraft is defined in FIDL using a sequence of flight segments, which consist of an ICDL composite describing the aircraft trajectory during that part of the flight. Semantics for the non-linear execution of flight segments are also provided.

A summary of the abstraction capabilities of the AIDL, ICDL, and FIDL is shown in figure 15.1. In the AIDL, no abstraction is allowed; trajectory descriptions must be unambiguous (save for changes in the weather conditions or the aircraft performance). In the ICDL some abstraction is allowed by leaving some elements (or even an entire profile) unspecified. Still, some information on the aircraft's behaviour must be specified. In the FIDL, it is possible to define the target state of the aircraft using a constraint, without specifying how that target state is achieved. Moreover, these three languages inherit all the expressive power of the languages they extend, which means that any trajectory described in AIDL can also be described in ICDL, and any trajectory described in the ICDL can be described in FIDL. This makes the FIDL the most flexible language for describing a single aircraft's trajectory.

However, during missions that involve multiple aircraft, the trajectories of 
those aircraft may be inter-dependant, specially if the aircraft work together towards some common goals. A language to describe multiple aircraft's trajectories and the dependencies between them was also deemed necessary. This kind of description is known as mission intent. The mission intent description language (MIDL) is a formal language built on top of the FIDL that can fulfil this necessity. A mission intent specified in MIDL comprises a number of tasks, constraints, and objectives. Tasks describe the trajectories that must be flown during the mission. A task may need to be completed after a particular event (known as the precondition of the task), and it may have to be completed several times. Also, there may be some requirements that the aircraft performing a task must meet (e.g., a particular type of aircraft or payload). The MIDL provides the means to specify this information. In addition to the tasks, mission constraints represent restrictions that affect one or more aircraft. Dependencies among different aircraft's trajectories can be specified using mission constraints. Finally, mission objectives represent global optimization goals that affect the whole mission. The MIDL completes the language hierarchy described in this thesis. Using the languages in this hierarchy, it is possible to describe the trajectories of one or more airborne aircraft with an arbitrary level of detail.

Since the initial goal was to be able to describe the whole flight, from push back to arrival at the gate, the means to describe aircraft operations on airport surface have also been developed. Another formal language, the surface movement description language (SMDL) was developed for this purpose. The main difference of the SMDL from the languages in the hierarchy is that the SMDL is based on the clearances used in surface operations instead of the equations of motion of the aircraft. The resulting language is simpler than the other languages described in this thesis. Nevertheless, the SMDL can be used to describe aircraft trajectories on airport surface with various levels of detail and specify some degree of uncertainty. A trajectory expressed in SMDL consists mainly on a list of instructions. Most instructions represent a clearance issued to the vehicle, but there are some special instructions that represent uncertainty (i.e., the trajectory may be partially undefined) or mutability (i.e., some instructions are subject to change in the future). Applications of this language have been mainly focused on the definition of safety nets [129].

Once the collection of languages was defined, the applications of these languages to aircraft management could be explored. The functions considered in this thesis are trajectory prediction and mission planning. In both of these functions, the concept of trajectory language processing engine, or simply engine, was used. An engine is a process whose main input and output are languages from the hierarchy described in this thesis.

A trajectory predictor can be formulated as a trajectory language processing engine where the input is either a flight intent (expressed in FIDL) or an aircraft intent (expressed in AIDL), and the output is a sampled trajectory. This engine was named trajectory prediction engine. It was also found that many of the components that compose this engine can also be seen as simpler engines. A modular trajectory predictor based on these ideas was also developed in collaboration with BR\&TE.

The trajectory prediction engine behaves differently depending on whether the input is an aircraft intent or a flight intent. In the first case, another engine, the trajectory computation engine is used to compute the aircraft intent and 
produce a sampled trajectory. The most interesting case, however, is that in which the input is a flight intent. In this case, the more abstract definition of the flight (i.e., the flight intent) must be translated in to an unambiguous description of the trajectory (i.e., an aircraft intent). This translation is carried out by the intent generation engine.

The intent generation engine performs two main tasks to generate an aircraft intent from a flight intent. The first task consists on generating an enriched flight intent that contains all the relevant information of the flight. This includes not only the information in the input flight intent, but also the relevant information from the user preferences model, the operational context model, and the aircraft performance model. This information is added by three chained engines: the user preferences engine, the operational context engine, and the aircraft performance limitations engine.

The second task performed by the intent generation engine is the translation of the enriched flight intent into an aircraft intent. This is the most important task in the intent generation engine, and it is carried out by an engine named intent generation core process engine. The intent generation core process engine uses the ICDL during the translation process. It first generates a composite describing the horizontal profile. This horizontal profile consists on a concatenation of great circles (orthodromes) and small circles (circular arcs), which take into account the speed constraints during different segments of the flight to compute the turn radii. For the vertical and configuration profiles, a search algorithm is used. This algorithm builds the vertical profile incrementally, adding manoeuvres along the flight to address the constraints in the flight. As there are many possible ways of addressing the constraints in the flight, a decision tree is used to explore the different possible trajectories. The decision tree is generated iteratively by a number of micro-strategies. In every iteration, each micro-strategy may generate a manoeuvre addressing one or more constraints. As different micro-strategies try different manoeuvres, the decision tree is populated with different possible trajectories. The process continues exploring the decision tree until a solution that successfully addresses all the constraints is found.

After the vertical/configuration profile generation is completed, the horizontal, vertical, and configuration profiles are combined into a single composite describing the whole flight. This ICDL composite may contain some parameters that can be optimized. A trajectory language processing engine can be used for this purpose. This engine, the composite optimization engine, uses optimization methods to compute the optimal combination of parameters. Once the optimization process concludes, the ICDL composite is translated into the AIDL by the composite translation engine, obtaining the output of the intent generation engine.

In the literature, most of the trajectory predictors are based on a predefined vertical/configuration profile, which is then amended to address other constraints in the flight. The approach in this thesis is a novel method that allows much more flexibility in the types of aircraft and the type of flights it can be applied to. The results obtained suggest that this method can provide results with the same quality as existing methods, while providing the extra flexibility. The caveat of this approach is the increase in the computation time, which is also very difficult to predict in some cases. 
The mission planning function was also studied in this thesis. A mission planner was formulated as an engine that translates a mission intent (expressed in MIDL) into a series of flight intents (expressed in FIDL), one for every available aircraft. The mission planning problem was formulated as a simple mixed integer linear programming problem that relies on a fast method to compute the cost of flying from one location to another. In the mixed integer linear programming formulation, the cost was related to the time it takes an aircraft to fly between two locations. In the absence of wind or aircraft performance information, the time is highly correlated to the length of the path between that two locations. For this reason, pre-computing the lengths of the paths for all the relevant locations in the mission was deemed a key problem in mission planning. A fast algorithm for computing near-shortest paths in an urban environment (where obstacles are most abundant) was developed, and a comparison with other approaches in the literature was performed.

\subsection{Contributions}

The contributions of this thesis can be summarized as the following:

- A variant of the AIDL was developed, which can be used to describe trajectories of quadrotor aircraft unambiguously. This variant, named QR-AIDL, was derived from the equations of motion of a quadrotor, and has similar features to those of the FW-AIDL. A prototype trajectory computation engine, which translates an aircraft intent expressed in QRAIDL into a sampled trajectory, was developed.

- Contributions to the development of the ICDL and FIDL have been made, in collaboration with BR\&TE, to make these languages reach the mature state they are today.

- The specification of a formal language for expressing mission intent, the MIDL. This language is different from previous approaches in that it is built on top of the FIDL and inherits its flexibility and expressive power.

- Collaboration in the implementation of the SMDL, a formal language for the description of trajectories of vehicles in the airport surface. This language is derived form the clearances that can be issued in this context.

- In collaboration with BR\&TE, development of the concept of trajectory language processing engine, and formulation of a trajectory prediction as an aggregation of these engines. This also included parts of a prototype implementation of a trajectory predictor based on these ideas.

- Also in collaboration with BR\&TE, the development of a novel approach to the generation of the vertical/configuration profiles in a trajectory predictor. The prototype mentioned above uses this approach.

- Formulation of the mission planning problem as a trajectory language processing engine that can be solved using mixed integer linear programming. This also motivated the development of a fast near-shortest path algorithm in an urban environment, which improves the performance of other algorithms in this particular scenario. 


\subsection{Further work}

Many of the works in this thesis open new lines of research. This section tries to emphasize those areas where new results would be particularly useful, as judged by the author.

In the development of the QR-AIDL, some difficulties were found in the integration of the equations of motion when the ground speed is zero (or, in practice, near zero). Using a different approach that solves (or better handles) this case may significantly improve the resulting language. This approach may involve a transformation of the equations of motion of the quadrotor, or giving them a different treatment. Also, the maturity of the QR-AIDL hasn't reached that of the FW-AIDL. For example, a thorough analysis of all the possible combinations of instructions hasn't been performed.

Some results regarding the use of the QR-AIDL have been provided. These results, however, are limited to simulated scenarios. In the future, tests in real situations could be performed to validate the results of this thesis with real data.

The language used to describe missions involving several aircraft, the MIDL, lacks support for defining the trajectory of an aircraft relative to that of another aircraft. Adding this possibility would allow, for example, describe flight formations. In the MIDL, it is not possible, in general, to describe tasks that involve several aircraft, which could be a future improvement to the language.

The applications of the SMDL have not been studied in this thesis. This language has been utilized for the generation of safety nets. Analyses of other applications, such as routing of vehicles in airport surface or conflict detection and resolution, could be conducted in the future.

The prototyped trajectory predictor described in this thesis could also be improved in several ways. In the vertical profile generation algorithm, it was found that the choice of micro-strategies and the 'most promising criterion' (used to determine which of the partial trajectories in the decision tree should be further developed) play a key role in the performance of the algorithm and the quality of its solution. These two elements should be fine-tuned to improve both metrics. Also, the application of a similar algorithm to the generation of the horizontal profile could be studied.

The approach used to solve the mission planning problem that is described in this thesis is quite limited. The described mission planner can only process a subset of all the missions that can be expressed in the MIDL, and the proposed solution (using a naive mixed integer linear programming formulation) is inefficient. While this served the purpose of emphasizing the importance of an efficient path planner, a more efficient solution would be needed if it was necessary to manage a mission in real time.

Although uncertainty has been taken into account in some particular subjects, many of the languages and applications covered in this thesis have considered simplified deterministic variants of the problems. Uncertainty is a key aspect in most ATM environments, and should be taken into account in future revisions of the languages described here. 



\section{Bibliography}

[1] John David Anderson. The Airplane, a history of its technology. Reston (Virginia), USA: American Institute of Aeronautics and Astronautics, 2002. 394 pp. ISBN: $978-1-56347-525-1$.

[2] Egbert Torenbeek and H. Wittenberg. Flight Physics: Essentials of Aeronautical Disciplines and Technology, with Historical Notes. Milton Keynes, UK: Springer Science+Business Media, 2009. 540 pp. ISBN: 978-1-4020-8663-2.

[3] Peter L. Jakab. 'Wood to Metal: The Structural Origins of the Modern Airplane'. In: Journal of Aircraft 36.6 (1999), pp. 914-918. ISSN: 00218669. DOI: $10.2514 / 2.2551$.

[4] Cary R. Spitzer, ed. Avionics: elements, software and functions. Second Edition. CRC Press, 2006, p. 448. ISBN: 978-0-8493-8439-4.

[5] Michael S. Nolan. Fundamentals of Air Traffic Control. Fifth Edition. Clifton Park (New York), USA: Cengage Learning, 2011. 674 pp. ISBN: 978-1-4354-8272-2.

[6] Richard P.G. Collinson. Introduction to Avionics. First Edition. Microwave Technology Series 11. London, UK: Chapman \& Hall, 1996. 472 pp. ISBN: $978-0-412-48250-2$.

[7] Air Transport Action Group. Revolutionising Air Traffic Management. May 2013. URL: http : / / www . atag - org / component / downloads / downloads/229.html.

[8] International Civil Aviation Organization (ICAO). Global Air Traffic Management Operational Concept. First Edition. Doc 9854. 2005. 82 pp.

[9] SESAR Consortium. Milestone Deliverable 3: The ATM Target Concept. SESAR Definition Phase DLM-0612-001-02-00. Sept. 2007, p. 108.

[10] Suraj G. Gupta, Mangesh M. Ghonge and P. M. Jawandhiya. 'Review of Unmanned Aircraft System (UAS)'. In: International Journal of Advanced Research in Computer Engineering 83 Technology 2.4 (Apr. 2013), pp. 1646-1658. ISSN: 2278-1323. URL: http://ijarcet.org/wpcontent/uploads/IJARCET-VOL-2-ISSUE-4-1646-1658.pdf.

[11] Alison George. 'Forget roads, drones are the future of goods transport'. In: New Scientist 219.2933 (7th Sept. 2013), p. 27. ISSN: 0262-4079. DOI: 10.1016/S0262-4079(13)62190-9. 
[12] Zak Sarris. 'Survey of UAV Applications in Civil Markets (June 2001)'. In: 9th IEEE Mediterranean Conference on Control and Automation. Dubrovnik, Croatia, June 2001, pp. 1-11.

[13] International Civil Aviation Organization (ICAO). Procedures for Air Navigation Services - Air Traffic Management. Fifteenth Edition. Doc 4444. 2007. $432 \mathrm{pp}$.

[14] John E. Hopcroft, Rajeev Motwani and Jeffrey D. Ullman. Introduction to Automata Theory, Languages, and Computation. Third Edition. Pearson Education, 2006. 750 pp. ISBN: 978-0-321-45536-9.

[15] Javier López-Leonés, Miguel A. Vilaplana, Eduardo Gallo, Francisco A. Navarro and Carlos Querejeta. 'The Aircraft Intent Description Language: A key enabler for air-ground synchronization in Trajectory-Based Operations'. In: The 26th IEEE/AIAA Digital Avionics Systems Conference. Oct. 2007, pp. 1.D.4-1-12. DOI: 10.1109/DASC. 2007.4391836.

[16] Javier López-Leonés. 'Definition of an aircraft intent description language for air traffic management applications'. PhD thesis. University of Glasgow, 3rd Nov. 2008. URL: http: //eleanor.lib.gla.ac.uk/ record=b2643400.

[17] Richard Bolczak, John C. Gonda III, William J. Saumsiegle and Ronald A. Tornese. 'Controller-pilot data link communications (CPDLC) Build 1 value-added services'. In: 23rd Digital Avionics Systems Conference. Vol. 1. Oct. 2004, pp. 2.D.1-9. DOI: 10.1109/DASC. 2004.1391272.

[18] RTCA. SC-214 Standards for Air Traffic Data Communication Services. 2015. URL: http : / / www . rtca . org / content . asp ? pl $=108 \& s l=33 \&$ contentid=83.

[19] EUROCAE. WG-78 Standards for Air Traffic Data Communication Services. 2015. URL: https://www. eurocae.net/wgs/active/?wg=WG-78.

[20] Michael R. C. Jackson, John Gonda, Rob Mead and Greg Saccone. 'The 4D trajectory data link (4DTRAD) service - Closing the loop for air traffic control'. In: Integrated Communications, Navigation and Surveillance Conference. May 2009, pp. 1-10. DOI: 10.1109/ICNSURV.2009.5172860.

[21] EUROCONTROL. Flight Object Interoperability Proposed Standard (FOIPS). 2015. URL: https://www.eurocontrol.int/services/foips.

[22] Mike Paglione, Carlos Garcia-Avello, Sip Swierstra, Robert Vivona and Steven Green. 'A collaborative approach to trajectory modeling validation'. In: The 24th IEEE/AIAA Digital Avionics Systems Conference. Vol. 1. Oct. 2005, pp. 3.C.4-1-12. DOI: 10.1109/DASC.2005.1563350.

[23] M. C. Waller, J. G. Rigopoulos, D. R. Blackman and T. F. Berreen. 'Considerations in the application of dynamic programming to optimal aircraft trajectory generation'. In: National Aerospace and Electronics Conference. Vol. 2. May 1990, pp. 574-579. DOI: 10.1109/NAECON. 1990. 112828.

[24] Andrea Lecchini, William Glover, John Lygeros and Jan Maciejowski. 'Air Traffic Control with an expected value criterion'. In: 16th IFAC World Congress. Prague, Czech Republic, July 2005, pp. 1-6. URL: http://scl. hanyang . ac . kr / scl / database / papers / IFAC / 2005 / Fullpapers / 01875.pdf. 
[25] Matt R. Jardin. 'Real-Time Conflict-Free Trajectory Optimization'. In: 5th USA/Europe Air Traffic Management RESD Seminar. Budapest, Hungary, June 2003, pp. 1-10. URL: http: // www . atmseminar . org/ seminarContent/seminar5/papers/p_027_TFO.pdf.

[26] Timothy R. Jorris and Richard G. Cobb. 'Multiple Method 2-D Trajectory Optimization Satisfying Waypoints and No-Fly Zone Constraints'. In: Journal of Guidance, Control, and Dynamics 31.3 (2008), pp. 543553. ISSN: 0731-5090. DOI: 10.2514/1.32354.

[27] Ick Ho Whang and Tae Won Hwang. 'Horizontal waypoint guidance design using optimal control'. In: IEEE Transactions on Aerospace and Electronic Systems 38.3 (July 2002), pp. 1116-1120. ISSN: 0018-9251. DOI: 10.1109/TAES. 2002.1039430.

[28] Shon Grabbe, Banavar Sridhar and Avijit Mukherjee. 'Sequential Traffic Flow Optimization with Tactical Flight Control Heuristics'. In: Journal of Guidance, Control, and Dynamics 32.3 (2009), pp. 810-820. ISSN: 07315090. DOI: $10.2514 / 1.40300$.

[29] H. G. Visser. A 4-D trajectory optimization and guidance technique for terminal area traffic management. Internal report LR-769. Delft University of Technology, Aug. 1994. URL: http:// repository . tudelft . $\mathrm{nl} /$ assets / uuid : 17b2cd01-8c0b-4395 - aadd - 1243f478b427 / LR 769.pdf.

[30] Ian Fairclough and David McKeever. PHARE Advanced Tools Trajectory Predictor. Final Report 98-70-18/10. Brussels, Belgium: EUROCONTROL, 2nd Aug. 2000, p. 50. URL: https://www . eurocontrol.int/ phare/gallery/content/public/documents/98-70-18-v10_tp.pdf.

[31] Marianne Jacobsen and Ulf T. Ringertz. 'Airspace Constraints in Aircraft Emission Trajectory Optimization'. In: Journal of Aircraft 47.4 (2010), pp. 1256-1265. ISSN: 0021-8669. DOI: 10.2514/1.47109.

[32] Mark B. Milam. 'Real-time optimal trajectory generation for constrained dynamical systems' PhD thesis. California Institute of Technology, 23rd May 2003. URL: http: / / resolver . caltech . edu / CaltechETD : etd-06022003-114340.

[33] Mario Innocenti, Lorenzo Pollini and Demetrio Turra. 'Guidance of Unmanned Air Vehicles Based on Fuzzy Sets and Fixed Waypoints'. In: Journal of Guidance, Control, and Dynamics 27.4 (2004), pp. 715-720. ISSN: 0731-5090. DOI: 10.2514/1.2651.

[34] Manuel Soler, Alberto Olivares and Ernesto Staffetti. 'Hybrid Optimal Control Approach to Commercial Aircraft Trajectory Planning'. In: Journal of Guidance, Control, and Dynamics 33.3 (2010), pp. 985-991. Issn: 0731-5090. DOI: $10.2514 / 1.47458$.

[35] Ioannis Lymperopoulos and John Lygeros. 'Improved Multi-Aircraft Ground Trajectory Prediction for Air Traffic Control'. In: Journal of Guidance, Control, and Dynamics 33.2 (2010), pp. 347-362. ISSN: 07315090. DOI: $10.2514 / 1.46190$. 
[36] Chaojie Zhang, Nan Wang and Jing Chen. 'Trajectory generation for aircraft based on differential flatness and spline theory'. In: International Conference on Information Networking and Automation. Vol. 1. Oct. 2010, pp. 110-114. DOI: 10.1109/ICINA.2010.5636425.

[37] Rushen B. Patel and Paul J. Goulart. 'Trajectory Generation for Aircraft Avoidance Maneuvers Using Online Optimization'. In: Journal of Guidance, Control, and Dynamics 34.1 (2011), pp. 218-230. ISSN: 0731-5090. DOI: $10.2514 / 1.49518$.

[38] Sip Swierstra. Common Trajectory Predictor Structure and Terminology in support of SESAR and NextGen. White paper. FAA/EUROCONTROL Action Plan 16, 29th Jan. 2010, pp. 1-25. URL: https://acy.tc.faa. gov/cpat/tjm/.

[39] Jinwhan Kim, Karthik Palaniappan, Padmanabhan Menon, Kamesh Subbarao and Manju Nag. 'Trajectory Uncertainty Modeling for Queueing Analysis of the NAS'. In: The 26th Congress of International Council of the Aeronautical Sciences. Anchorage (Alaska), USA: American Institute of Aeronautics and Astronautics, Sept. 2008. DOI: 10.2514/6.20088854.

[40] Colin Meckiff, Renaud Chone and Jean-Pierre Nicolaon. 'The tactical load smoother for multi-sector planning'. In: The 2nd USA/Europe Air Traffic Management RESD seminar. Orlando (Florida), USA, Dec. 1998. URL: http : / / www . atmseminarus . org / seminarContent / seminar2 / papers/p_040_FOC.pdf.

[41] David Knorr and Leif Walter. 'Trajectory Uncertainty and the Impact on Sector Complexity and Workload'. In: 1st SESAR Innovation Days. Toulouse, France, Nov. 2011, pp. 1-8. ISBN: 978-2-87497-024-5. URL: http : / / www . sesarinnovationdays . eu / files / SIDs / SID\% 202011 04.pdf.

[42] International Civil Aviation Organization (ICAO). Manual on Air Traffic Management System Requirements. First Edition. Doc 9882. 2008. 67 pp.

[43] S.A.N. Magill and P.A. Platt. 'A fast-time simulation study of shared aircraft intent information'. In: The 21st Digital Avionics Systems Conference. Vol. 1. Irvine (California), USA, Oct. 2002, 2A4-1-10. DoI: 10. 1109/DASC. 2002.1067911.

[44] Rob Ruigrok and Mario Valenti Clari. 'The impact of aircraft intent information and traffic separation assurance responsibility on en-route airspace capacity'. In: 5th USA/Europe Air Traffic Management R\&D Seminar. Budapest, Hungary, June 2003, pp. 1-11. URL: http://www . atmseminar.org/seminarContent/seminar5/papers/p_055_AGC.pdf.

[45] Stephane Mondoloni and Sip Swierstra. 'Commonality in disparate trajectory predictors for air traffic management applications'. In: The 24th IEEE/AIAA Digital Avionics Systems Conference. Vol. 1. Oct. 2005, pp. 3.C.2-1-10. DOI: 10.1109/DASC.2005.1563348. 
[46] Miguel A. Vilaplana, Javier López-Leonés, Ernesto Valls and Francisco A. Navarro. COURAGE Domain Analysis. BRTE-ATM0536-COU2001DA. Madrid, Spain: EUROCONTROL, 15th Apr. 2005. URL: http : / / library . atmrt . org / lib2 / 2006 / COURAGE / COURAGE \% 20Domain \% 20Analysis.pdf.

[47] Miguel A. Vilaplana, Eduardo Gallo, Francisco A. Navarro and Sip Swierstra. 'Towards a formal language for the common description of aircraft intent'. In: The 24th IEEE/AIAA Digital Avionics Systems Conference. Vol. 1. Oct. 2005, pp. 3.C.5-1-9. ISBN: 0-7803-9307-4. DOI: 10.1109 / DASC. 2005.1563351.

[48] Javier López-Leonés, Enrique J. Casado, Miguel A. Vilaplana and Francisco A. Navarro. 'Providing Data for Predicting Aircraft Trajectory'. US Patent 9153136. The Boeing Company. 6th Oct. 2015.

[49] Alan Lee, Xavier Bouyssounouse and James Murphy. 'The Trajectory Synthesizer Generalized Profile Interface'. In: 10th AIAA Aviation Technology, Integration, and Operations Conference. American Institute of Aeronautics and Astronautics, Sept. 2010. DOI: 10.2514/6.2010-9138.

[50] C. Leclaire, P. Q. Nguyen and T. Mandon. CITRAC: Common Interface for TRAjectory Computation. CENA/NT95147. Orly, France: Centre d'études de la navigation aérienne (CENA), Apr. 1995, p. 49.

[51] John N. Barrer. 'The concept of path objects'. In: 18th Digital Avionics Systems Conference. Vol. 1. St. Louis (Missouri), USA, Oct. 1999, 5.B.21-7. ISBN: 0-7803-5749-3. DOI: 10.1109/DASC.1999.863740.

[52] John N. Barrer. 'Integrating the Cockpit with Air Traffic Management: the Concept of Path Objects'. In: 3rd USA/Europe Air Traffic Management RGD Seminar. Napoli, Italy, June 2000, pp. 1-6. URL: http: //www . atmseminar . org/seminarContent/seminar3/papers/p_074_ DSTCDM.pdf.

[53] Enrique J. Casado, Francisco A. Navarro, Juan A. Besada, Javier LópezLeonés and Miguel A. Vilaplana. 'Providing a Description of Aircraft Intent'. US Patent 8744649. The Boeing Company. 3rd June 2014.

[54] Xiao-Bing $\mathrm{Hu}$, Shu-Fan Wu and Ju Jiang. 'On-line free-flight path optimization based on improved genetic algorithms'. In: Engineering Applications of Artificial Intelligence 17.8 (Dec. 2004), pp. 897-907. IssN: 0952-1976. DOI: 10.1016/j.engappai.2004.08.015.

[55] Eduard Santamaría, Pablo Royo, Juan López, Cristina Barrado, Enric Pastor and Xavier Prats. 'Increasing UAV capabilities through autopilot and flight plan abstraction'. In: IEEE/AIAA 26th Digital Avionics Systems Conference. Dallas (Texas), USA, 21st Oct. 2007, 5.B.5-1-10. DOI: 10.1109/DASC. 2007.4391935.

[56] Enric Pastor, Eduard Santamaria, Pablo Royo, Juan Lopez and Cristina Barrado. 'On the design of a UAS flight plan monitoring and edition system'. In: IEEE Aerospace Conference. Big Sky (Montana), USA, Mar. 2010, pp. 1-20. DOI: 10.1109/AERO.2010.5446778.

[57] EUROCONTROL and FAA. Aeronautical Information Exchange Model. Feb. 2006. URL: http://www. aixm.aero/. 
[58] Aeronautical Radio, Inc. ARINC Specification 424-20: Navigation System Database. Specification. Dec. 2011.

[59] Angela Nuic. User Manual for the Base of Aircraft Data (BADA) Revision 3.12. Technical/Scientific Report 14/04/24-44. EUROCONTROL Experimental Centre, Aug. 2014. URL: https://www . eurocontrol.int/ services/bada.

[60] EFMS Integration Team. EFMS Phase 1B - Technical Reference Document. 96-70-15. Brussels, Belgium: EUROCONTROL, 7th Nov. 1996, p. 242. URL: http://www. eurocontrol.int/phare/gallery/content/ public/documents/96-70-15EFMS1bTRD.pdf.

[61] Bill Roberson. 'Fuel Conservation Strategies: Cost Index Explained'. In: AERO Magazine (QTR_2.07 2007), pp. 26-28. URL: http://www . boeing . com/commercial/aeromagazine/articles/qtr_2_07/AERO_ Q207_article5.pdf.

[62] Andreas Tolk, Kevin Galvin, Michael Hieb and Lionel Khimeche. 'Coalition Battle Management Language'. In: Fall Simulation Interoperability Workshop. Orlando (Florida), USA: DTIC Document, Sept. 2004, pp. 112. URL: https ://www . sisostds . org/DesktopModules/Bring2mind/ DMX/Download . aspx?Command=Core_Download\&EntryId=25668.

[63] Curtis Blais, Dick Brown, Saikou Diallo, Kevin Heffner, Stan Levine, Samuel Singapogu and Marc St-Onge. 'Coalition Battle Management Language (C-BML) Phase 1 Specification Development: An Update to the M\&S Community'. In: Fall Simulation Interoperability Workshop. Orlando (Florida), USA, 2009, pp. 1-9. URL: https://www. sisostds .org/ DesktopModules / Bring2mind / DMX / Download . aspx ? Command = Core _ Download\&Entry Id=28668.

[64] Michael Wunder. Concept for a SocialC2IS - Bridging the Heterogeneity of People and IT. Tech. Report RTO-MP-HFM-201. Wachtberg, Germany: Fraunhofer -FKIE, Apr. 2012, p. 12. URL: http://ftp.rta.nato. int/public/pubfulltext/rto/mp\%5crto-mp-hfm-201/mp-hfm-20108. doc.

[65] NASA Intelligent Systems Division. Apex. 2006. URL: http : / / apexautonomy. sourceforge. net/.

[66] Patrick Doherty, Fredrik Heintz and Jonas Kvarnström. 'High-Level Mission Specification and Planning for Collaborative Unmanned Aircraft Systems Using Delegation'. In: Unmanned Systems 01.1 (22nd May 2013), pp. 75-119. ISSN: 2301-3850. DOI: 10.1142/S2301385013500052.

[67] Sertac Karaman and Emilio Frazzoli. 'Linear temporal logic vehicle routing with applications to multi-UAV mission planning'. In: International Journal of Robust and Nonlinear Control 21.12 (Aug. 2011), pp. 13721395. ISSN: 1099-1239. DOI: 10.1002/rnc.1715.

[68] Eskindir Asmare, Naranker Dulay, Hahnsang Kim, Emil C. Lupu and Morris Sloman. 'Management architecture and mission specification for unmanned autonomous vehicles'. In: 1st Systems Engineering for Autonomous Systems Defence Technology Centre Technical Conference. Edinburgh (Scotland), UK, July 2006, pp. 1-8. URL: http://pubs.doc.ic. ac.uk/SEAS06/SEAS06.pdf. 
[69] Miaobo Dong, Ben M. Chen, Guowei Cai and Kemao Peng. 'Development of a Real-time Onboard and Ground Station Software System for a UAV Helicopter'. In: Journal of Aerospace Computing, Information, and Communication 4.8 (2007), pp. 933-955. DOI: 10.2514/1.26408.

[70] Eduard Santamaría. 'Formal Mission Specification and Execution Mechanisms for Unmanned Aircraft Systems'. PhD thesis. Universitat Politècnica de Catalunya, June 2010. 151 pp. URL: http://www.tdx . cat/ handle/10803/6020.

[71] David Harel and Michal Politi. Modeling Reactive Systems with Statecharts: The Statemate Approach. 1st. New York (NY), USA: McGrawHill, Inc., 1998. ISBN: 978-0-07-026205-8.

[72] Tadao Murata. 'Petri nets: Properties, analysis and applications'. In: Proceedings of the IEEE 77.4 (1989), pp. 541-580. ISSN: 0018-9219. DOI: 10.1109/5.24143.

[73] Magali Barbier and Elodie Chanthery. 'Autonomous mission management for unmanned aerial vehicles'. In: Aerospace Science and Technology 8.4 (June 2004), pp. 359-368. ISSN: 1270-9638. DOI: $10.1016 / \mathrm{j}$. ast. 2004.01 .003$.

[74] Iván Maza, Jorge Muñoz-Morera, Fernando Caballero, Enrique J. Casado, Víctor Pérez-Villar and Aníbal Ollero. 'Architecture and tools for the generation of Flight Intent from mission intent for a fleet of Unmanned Aerial Systems'. In: International Conference on Unmanned Aircraft Systems. May 2014, pp. 9-19. DOI: 10.1109/ICUAS. 2014.6842234.

[75] Gerd Bumiller, Lutz Lampe and Halid Hrasnica. 'Power line communication networks for large-scale control and automation systems'. In: IEEE Communications Magazine 48.4 (Apr. 2010), pp. 106-113. ISSN: 01636804. DOI: 10.1109/MCOM. 2010.5439083.

[76] Keyvan Bayram, Theo Hankers, Nico Zimmer, Jens Schiefele and P. Hecker. 'Situation awareness through the integration of transient information into future cockpit taxi guidance'. In: 30th Digital Avionics Systems Conference. Oct. 2011, 8E1-1-9. DOI: 10.1109/DASC.2011.6096154.

[77] Steven M. Green, Sip Swierstra, Stephane Mondoloni, Richard Irvine, Mike Paglione and Carlos García-Avello. Common Methodology and Resources for the Validation and Improvement of Trajectory Prediction Capabilities. White paper. FAA/EUROCONTROL Action Plan 16, 2003, pp. 1-17. URL: https://acy.tc.faa.gov/cpat/tjm/white_paper_V1. $0 . p d f$.

[78] Eduardo Gallo. 'Prediction of descent trajectories based on Aircraft Intent'. In: The 29th IEEE/AIAA Digital Avionics Systems Conference. Salt Lake City (Utah), USA, Oct. 2010, pp. 2.D.1-1-16. DoI: 10.1109/ DASC. 2010.5655487.

[79] Jean-Louis Renteux. Aircraft Modelling Standards for Future ATC Systems. 872003. Brussels, Belgium: EUROCONTROL Division E1, July 1987.

[80] Eric Hoffman. 'Contribution to Aircraft Performance Modeling for ATC'. In: Air Traffic Control Quarterly 2.2 (1994), pp. 103-130. 
[81] Alexander Suchkov, Angela Nuic and Sip Swierstra. 'Aircraft Performance Modeling For Air Traffic Management Applications'. In: 5th USA/Europe Air Traffic Management RED Seminar. Budapest, Hungary, June 2003, pp. 1-10. URL: http : / / www . atmseminar . org / seminarContent/seminar5/papers/p_039_DS.pdf.

[82] Karen M. Feigh. 'An Airspace Simulator for Air Traffic management Research'. Master's Thesis. Cranfield, UK: Cranfield University, Nov. 2003. 253 pp. URL: http://www2.isye.gatech.edu/ kfeigh/publications/ Feigh_Mphil_Thesis.pdf.

[83] Rhonda A. Slattery and Yiyuan J. Zhao. 'Trajectory Synthesis for Air Traffic Automation'. In: Journal of Guidance, Control, and Dynamics 20.2 (Mar. 1997), pp. 232-238. ISSN: 0731-5090. DOI: 10.2514/2.4056.

[84] Mick van Gool and Helmut Schröter. PHARE Final Report. Final Report 99-70-09. Brussels, Belgium: Eurocontrol, Nov. 1999, p. 67. URL: http:// www . eurocontrol.int/phare/gallery/content/public/documents/ 99-70-09pharefinal10.pdf.

[85] William Gill and Robert Maddock. Prediction of Optimal $4 D$ Trajectories in the Presence of Time and Altitude Constraints. 97-70-09. Brussels, Belgium: Eurocontrol, 15th Feb. 1997, p. 29. URL: http://www . eurocontrol . int/phare/gallery/content/public/documents /9770-09efms_4d_trajectory_prediction.pdf.

[86] Randy Walter. 'Flight Management Systems'. In: Avionics: elements, software and functions. Ed. by Cary R. Spitzer. Second Edition. CRC Press, 2006, pp. 20-1-20-26. ISBN: 978-0-8493-8439-4.

[87] Huo Yan, Cheng Nong and Li Qing. 'Design and simulation of vertical trajectory predictions in flight management system'. In: IEEE Chinese Guidance, Navigation and Control Conference. 2014, pp. 2187-2191. DOI: 10.1109/CGNCC. 2014.7007512.

[88] David H. Williams and Charles E. Knox. '4D Descent Trajectory Generation Techniques Under Realistic Operating Conditions'. In: Aircraft Trajectories Computation-Prediction-Control. Ed. by André Benoît. Vol. 2. AGARDograph 301. Loughton, UK, May 1990, pp. 25-1-25-22. ISBN: 92835-0562-X.

[89] Alessandro Gardi, Roberto Sabatini, Subramanian Ramasamy and Trevor Kistan. 'Real-Time Trajectory Optimisation Models for Next Generation Air Traffic Management Systems'. In: Applied Mechanics and Materials 629 (Oct. 2014), pp. 327-332. IssN: 1662-7482. DOI: 10.4028/www. scientific.net/AMM. 629.327.

[90] Yao-Hong Qu, Quan Pan and Jian-guo Yan. 'Flight path planning of UAV based on heuristically search and genetic algorithms'. In: 31st Annual Conference of IEEE Industrial Electronics Society. Raleigh (North Carolina), USA, Nov. 2005, pp. 45-49. DOI: 10.1109/IECON.2005.1568876.

[91] George Vachtsevanos, Liang Tang, Graham Drozeski and Luis Gutierrez. 'From mission planning to flight control of unmanned aerial vehicles: Strategies and implementation tools'. In: Annual Reviews in Control 29.1 (2005), pp. 101-115. ISSN: 1367-5788. DOI: 10.1016/j.arcontrol. 2004. 11.002 . 
[92] Mark J. Nelson. UAV mission planning. Technical Report DSTO-TR0164. Salisbury, Australia: Defence Science and Technology Organisation, June 1995, p. 38. URL: http://dspace.dsto.defence.gov.au/dspace/ handle/1947/4350.

[93] Tania Bedrax-Weiss, Conor McGann and Michael Iatauro. 'EUROPA 2: Plan Database Services for planning and Scheduling Applications'. In: International Conference on Automated Planning and Scheduling. System Demonstrations. Monterey (California), USA, 6th Aug. 2005, pp. 18-19. URL: http://icaps05.icaps-conference.org/documents/ proceedings/sd-allpapers.pdf.

[94] Javier Barreiro et al. 'EUROPA: A platform for AI planning, scheduling, constraint programming, and optimization'. In: 22nd International Conference on Automated Planning and Scheduling. Sao Paulo, Brazil, June 2012.

[95] Gilbert Laporte and Silvano Martello. 'The selective travelling salesman problem'. In: Discrete Applied Mathematics 26.2 (1990), pp. 193-207. ISSN: 0166-218X. DOI: 10.1016/0166-218X (90)90100-Q.

[96] Lanah Evers, Twan Dollevoet, Ana Isabel Barros and Herman Monsuur. 'Robust UAV mission planning'. In: Annals of Operations Research 222.1 (11th Dec. 2012), pp. 293-315. ISSN: 0254-5330, 1572-9338. DOI: 10.1007/ s10479-012-1261-8.

[97] Adam J. Pohl and Gary B. Lamont. 'Multi-objective UAV Mission Planning Using Evolutionary Computation'. In: Proceedings of the 40th Conference on Winter Simulation. Miami (Florida), USA, Dec. 2008, pp. 1268-1279. ISBN: 978-1-4244-2708-6. URL: http : // dl . acm . org / citation. cfm?id=1516744.1516965.

[98] Yoonsoo Kim, Da-Wei Gu and Ian Postlethwaite. 'Real-Time Optimal Mission Scheduling and Flight Path Selection'. In: IEEE Transactions on Automatic Control 52.6 (June 2007), pp. 1119-1123. ISSN: 0018-9286. DOI: $10.1109 / \mathrm{TAC} .2007 .899048$.

[99] Stephen Leary, Markus Deittert and John Bookless. 'Constrained UAV mission planning: A comparison of approaches'. In: IEEE International Conference on Computer Vision Workshops. Barcelona, Spain, Nov. 2011, pp. 2002-2009. DOI: 10.1109/ICCVW. 2011.6130494.

[100] Ozgur Koray Sahingoz. 'Flyable path planning for a multi-UAV system with Genetic Algorithms and Bezier curves'. In: International Conference on Unmanned Aircraft Systems. Atlanta (Georgia), USA, May 2013, pp. 41-48. DOI: 10.1109/ICUAS. 2013.6564672.

[101] Mark de Berg, Marc van Kreveld, Mark Overmars and Otfried Cheong. Computational Geometry: Algorithms and Applications. 3rd ed. Springer Berlin Heidelberg, 2008. ISBN: 978-3-642-09681-5.

[102] Donald E. Knuth. 'Big Omicron and big Omega and big Theta'. In: SIGACT News 8.2 (Apr. 1976), pp. 18-24. ISSN: 0163-5700. DOI: 10 . 1145/1008328.1008329.

[103] Takao Asano, Tetsuo Asano, Leonidas Guibas, John Hershberger and Hiroshi Imai. 'Visibility of disjoint polygons'. In: Algorithmica 1.1 (Nov. 1986), pp. 49-63. ISSN: 0178-4617, 1432-0541. DOI: 10.1007/BF01840436. 
[104] Emo Welzl. 'Constructing the visibility graph for $n$-line segments in $O\left(n^{2}\right)$ time'. In: Information Processing Letters 20.4 (10th May 1985), pp. 167-171. ISSN: 0020-0190. DOI: 10.1016/0020-0190 (85) 90044-4.

[105] Subir Kumar Ghosh and David M. Mount. 'An output sensitive algorithm for computing visibility graphs'. In: 28th Annual Symposium on Foundations of Computer Science. 1987, pp. 11-19. DOI: 10.1109/SFCS.1987.6.

[106] John Hershberger and Subhash Suri. 'An Optimal Algorithm for Euclidean Shortest Paths in the Plane'. In: SIAM Journal on Computing 28.6 (Jan. 1999), pp. 2215-2256. ISSN: 0097-5397, 1095-7111. DOI: $10.1137 / \mathrm{S} 0097539795289604$.

[107] Leonidas Guibas, John Hershberger, Daniel Leven, Micha Sharir and Robert E. Tarjan. 'Linear-time algorithms for visibility and shortest path problems inside triangulated simple polygons'. In: Algorithmica 2.1 (Nov. 1987), pp. 209-233. ISSN: 0178-4617, 1432-0541. DOI: $10.1007 /$ BF01840360.

[108] Rosli Omar and Da-Wei Gu. 'Visibility line based methods for UAV path planning'. In: ICROS-SICE International Joint Conference. Fukuoka, Japan, Aug. 2009, pp. 3176-3181. ISBN: 978-4-907764-34-0.

[109] Pankaj K. Agarwal, R. Sharathkumar and Hai Yu. 'Approximate Euclidean Shortest Paths Amid Convex Obstacles'. In: 20th Annual ACMSIAM Symposium on Discrete Algorithms. New York (New York), USA: Society for Industrial and Applied Mathematics, Jan. 2009. URL: http: //dl.acm.org/citation. cfm?id=1496770.1496802.

[110] Kenneth L. Clarkson. 'Approximation Algorithms for Shortest Path Motion Planning'. In: 19th Annual ACM Symposium on Theory of Computing. New York (New York), USA, May 1987, pp. 56-65. ISBN: 0-89791221-7. DOI: $10.1145 / 28395.28402$.

[111] L. Paul Chew. 'There are planar graphs almost as good as the complete graph'. In: Journal of Computer and System Sciences 39.2 (Oct. 1989), pp. 205-219. ISSN: 0022-0000. DOI: 10.1016/0022-0000 (89)90044-5.

[112] John Canny and John H. Reif. 'New lower bound techniques for robot motion planning problems'. In: 28th Annual Symposium on Foundations of Computer Science. Los Angeles (California), USA, Oct. 1987, pp. 4960. ISBN: 0-8186-0807-2. DOI: 10.1109/SFCS.1987.42.

[113] John H. Reif and James A. Storer. 'A Single-exponential Upper Bound for Finding Shortest Paths in Three Dimensions'. In: Journal of the ACM 41.5 (Sept. 1994), pp. 1013-1019. ISSN: 0004-5411. DOI: 10.1145/185675. 185811.

[114] David M. Mount. On Finding Shortest Paths on Convex Polyhedra. Tech. Report. Computer Science Department, University of Maryland, May 1985, pp. 1-30. URL: http:// www . cs . umd . edu/ mount/Papers/spconvex.pdf.

[115] Micha Sharir and Avikam Baltsan. 'On Shortest Paths Amidst Convex Polyhedra'. In: 2nd Annual Symposium on Computational Geometry. Yorktown Heights (New York), USA, June 1986, pp. 193-206. ISBN: 0-89791-194-6. DOI: 10.1145/10515.10537. 
[116] Laxmi P. Gewali, Simeon Ntafos and Ioannis G. Tollis. 'Path planning in the presence of vertical obstacles'. In: IEEE Transactions on Robotics and Automation 6.3 (June 1990), pp. 331-341. ISSN: 1042-296X. DOI: 10. $1109 / 70.56665$.

[117] Tomás Lozano-Pérez and Michael A. Wesley. 'An Algorithm for Planning Collision-free Paths Among Polyhedral Obstacles'. In: Communications of the ACM 22.10 (Oct. 1979), pp. 560-570. ISSN: 0001-0782. DOI: 10 . $1145 / 359156.359164$.

[118] Christos H. Papadimitriou. 'An algorithm for shortest-path motion in three dimensions'. In: Information Processing Letters 20.5 (12th June 1985), pp. 259-263. ISSN: 0020-0190. DOI: 10 . 1016/0020-0190(85) 90029-8.

[119] Joonsoo Choi, Jürgen Sellen and Chee-Keng Yap. 'Approximate Euclidean Shortest Path in 3-space'. In: 10th Annual Symposium on Computational Geometry. Stony Brook (New York), USA, June 1994, pp. 41-48. ISBN: 0-89791-648-4. DOI: 10.1145/177424.177501.

[120] Joonsoo Choi, Jürgen Sellen and Chee-Keng Yap. 'Precision-sensitive Euclidean Shortest Path in 3-space'. In: 11th Annual Symposium on Computational Geometry. Vancouver, Canada, June 1995, pp. 350-359. ISBN: 0-89791-724-3. DOI: 10.1145/220279.220317.

[121] Zhang Qi, Zhenhai Shao, Yeo Swee Ping, Lim Meng Hiot and Yew Kong Leong. 'An Improved Heuristic Algorithm for UAV Path Planning in 3D Environment'. In: 2nd International Conference on Intelligent HumanMachine Systems and Cybernetics. Vol. 2. Nanjing (Jiangsu), China, Aug. 2010, pp. 258-261. ISBN: 978-1-4244-7869-9. DOI: 10.1109/IHMSC. 2010. 165.

[122] Luca De Filippis, Giorgio Guglieri and Fulvia Quagliotti. 'Path Planning Strategies for UAVS in 3D Environments'. In: Journal of Intelligent 8 Robotic Systems 65.1 (Jan. 2012), pp. 247-264. ISSN: 0921-0296, 15730409. DOI: $10.1007 / \mathrm{s} 10846-011-9568-2$.

[123] Rosli Omar and Da-Wei Gu. '3D Path Planning for Unmanned Aerial Vehicles using Visibility Line based Method'. In: 7th International Conference on Informatics in Control, Automation and Robotics. Vol. 1. Funchal (Madeira), Portugal, June 2010, pp. 80-85. ISBN: 978-989-8425-00-3.

[124] Ettore De Lellis, Gianfranco Morani, Federico Corraro and Vittorio Di Vito. 'On-line trajectory generation for autonomous unmanned vehicles in the presence of no-fly zones'. In: Proceedings of the Institution of Mechanical Engineers, Part G: Journal of Aerospace Engineering 227.2 (Feb. 2013), pp. 381-393. ISSN: 0954-4100, 2041-3025. DOI: 10.1177 / 0954410011430173.

[125] Cheng Xiao-dong, Zhou De-yun and Zhang Ruo-nan. 'New method for UAV online path planning'. In: IEEE International Conference on Signal Processing, Communication and Computing. Kunming (Yunnan), China, Aug. 2013, pp. 1-5. DOI: 10.1109/ICSPCC.2013.6664044. 
[126] V. Roberge, M. Tarbouchi and G. Labonte. 'Comparison of Parallel Genetic Algorithm and Particle Swarm Optimization for Real-Time UAV Path Planning'. In: IEEE Transactions on Industrial Informatics 9.1 (Feb. 2013), pp. 132-141. ISSN: 1551-3203. DOI: 10.1109/TII.2012.2198665.

[127] Juan A. Besada, Guillermo Frontera, Jesús Crespo, Enrique J. Casado and Javier López-Leonés. 'Automated Aircraft Trajectory Prediction Based on Formal Intent-Related Language Processing'. In: IEEE Transactions on Intelligent Transportation Systems 14.3 (4th June 2013), pp. 1067-1082. ISSN: 1524-9050. DOI: 10.1109/TITS. 2013.2252343.

[128] Guillermo Frontera, Juan A. Besada, Ana M. Bernardos, Enrique J. Casado and Javier López-Leonés. 'Formal Intent-Based Trajectory Description Languages'. In: IEEE Transactions on Intelligent Transportation Systems 15.4 (20th Feb. 2014), pp. 1550-1566. ISSN: 1524-9050. DOI: 10.1109/TITS. 2014.2299971.

[129] Guillermo Frontera, Juan A. Besada, Rafael de Andrea and David J. Martín. 'Airport routing and safety nets based on standardized surface movement description'. In: The 30th IEEE/AIAA Digital Avionics Systems Conference. Seattle (Washington), USA, 2011, pp. 1-17. DOI: 10. 1109/DASC. 2011.6096308.

[130] Ahmed F. Tarhan, Emre Koyuncu, Mehmet Hasanzade, Ugur Ozdemir and Gokhan Inalhan. 'Formal intent based Flight Management System design for unmanned aerial vehicles'. In: International Conference on Unmanned Aircraft Systems. May 2014, pp. 984-992. DOI: 10.1109 / ICUAS . 2014.6842349.

[131] Earl A. Coddington and Norman Levinson. Theory of Ordinary Differential Equations. Krieger Pub Co., June 1984. 436 pp. ISBN: 9780898747553.

[132] John D. Pryce. 'A Simple Structural Analysis Method for DAEs' In: BIT Numerical Mathematics 41.2 (Mar. 2001), pp. 364-394. ISSN: 0006-3835, 1572-9125. DOI: 10.1023/A:1021998624799.

[133] Samir Bouabdallah and Roland Siegwart. 'Full control of a quadrotor'. In: 2007 IEEE/RSJ International Conference on Intelligent Robots and Systems. San Diego (California), USA, Oct. 2007, pp. 153-158. DOI: 10. 1109/IROS. 2007.4399042.

[134] Samir Bouabdallah. 'Design and Control of Quadrotors with application to autonomous flying'. PhD thesis. École polytechnique fédérale de Lausanne, 23rd Feb. 2007. DOI: 10.5075/epfl-thesis-3727.

[135] Rahul Goel, Sapan M. Shah, Nitin K. Gupta and N. Ananthkrishnan. 'Modeling, simulation and flight testing of an autonomous quadrotor'. In: IISc Centenary International Conference and Exhibition on Aerospace Engineering. Bangalore, India, May 2009, pp. 18-22. URL: http://www. idearesearch. in/Papers/GN-070.pdf.

[136] Bora Erginer and Erdinç Altuğ. 'Modeling and PD Control of a Quadrotor VTOL Vehicle'. In: 2007 IEEE Intelligent Vehicles Symposium. Istanbul, Turkey, June 2007, pp. 894-899. DOI: 10.1109/IVS.2007.4290230. 
[137] Abdellah Mokhtari and A. Benallegue. 'Dynamic feedback controller of Euler angles and wind parameters estimation for a quadrotor unmanned aerial vehicle'. In: 2004 IEEE International Conference on Robotics and Automation. Vol. 3. New Orleans (Louisiana), USA, Apr. 2004, pp. 23592366. DOI: 10.1109/ROBOT. 2004.1307414.

[138] Armando S. Sanca, Pablo J. Alsina and Jés de Jesus F. Cerqueira. 'Dynamic Modelling of a Quadrotor Aerial Vehicle with Nonlinear Inputs'. In: IEEE Latin American Robotic Symposium. Natal, Brazil, Oct. 2008, pp. 143-148. DOI: 10.1109/LARS.2008.17.

[139] Xiaodong Zhang, Xiaoli Li, Kang Wang and Yanjun Lu. 'A Survey of Modelling and Identification of Quadrotor Robot'. In: Abstract and Applied Analysis 2014 (20th Oct. 2014), pp. 1-16. ISSN: 1085-3375. DOI: $10.1155 / 2014 / 320526$.

[140] Samir Bouabdallah, Pierpaolo Murrieri and Roland Siegwart. 'Design and control of an indoor micro quadrotor'. In: 2004 IEEE International Conference on Robotics and Automation. Vol. 5. New Orleans (Louisiana), USA, Apr. 2004, pp. 4393-4398. DOI: 10.1109/ROBOT.2004.1302409.

[141] Atheer L. Salih, M. Moghavvemi, Haider A. F. Mohamed and Khalaf Sallom Gaeid. 'Modelling and PID controller design for a quadrotor unmanned air vehicle'. In: 2010 IEEE International Conference on Automation Quality and Testing Robotics. Vol. 1. Cluj-Napoca, Romania, May 2010, pp. 1-5. DOI: 10.1109/AQTR. 2010.5520914.

[142] P. Pounds, R. Mahony and P. Corke. 'Modelling and control of a large quadrotor robot'. In: Control Engineering Practice. Special Issue on Aerial Robotics 18.7 (July 2010), pp. 691-699. ISSN: 0967-0661. DOI: 10.1016/j.conengprac. 2010.02.008.

[143] Kathryn E. Brenan, Stephen L. Campbell and Linda R. Petzold. Numerical Solution of Initial-Value Problems in Differential-Algebraic Equations. SIAM, 1996. 268 pp. ISBN: 978-0-898713-53-4.

[144] Javier López-Leonés, Miguel A. Vilaplana, Ibrahim Bayraktutar, Joel K. Klooster, José Manuel Asensio, Greg McDonald and Peter Kappertz. 'Towards an open test bed for the study of trajectory synchronization in the future ATM system: The ASIS initiative'. In: 2009 Integrated Communications, Navigation and Surveillance Conference. Arlington (Virginia), USA, May 2009, pp. 1-14. ISBN: 978-1-4244-4733-6. DOI: 10.1109/ ICNSURV . 2009. 5172825.

[145] Enrique J. Casado, Juan A. Besada, Javier López-Leonés, Ana M. Bernardos, Guillermo Frontera and Francisco A. Navarro. 'Providing a Description of Aircraft Intent'. US Patent 8977411. The Boeing Company. 10th Mar. 2015.

[146] ENAIRE. Aeronautical Information Publication Spain. 2nd Apr. 2015. URL: http : / / www . enaire.es / csee/Satellite/navegacion-aerea/ en/Page/1078418725020/.

[147] Javier López-Leonés, Enrique J. Casado, Juan A. Besada and Guillermo Frontera. 'Providing a Description of Aircraft Intent'. US Patent 9135828. The Boeing Company. 15th Sept. 2015. 
[148] Edsger W. Dijkstra. 'A note on two problems in connexion with graphs'. In: Numerische Mathematik 1.1 (1st Dec. 1959), pp. 269-271. ISSN: 0029599X. DOI: 10.1007/BF01386390.

[149] Donald E. Knuth. 'A generalization of Dijkstra's algorithm'. In: Information Processing Letters 6.1 (Feb. 1977), pp. 1-5. ISSN: 0020-0190. DOI: 10.1016/0020-0190 (77) 90002-3.

[150] Enrique J. Casado, Juan A. Besada, Javier López-Leonés, Jesús Crespo and Guillermo Frontera. 'Providing a Description of Aircraft Intent'. US Patent 8798813. The Boeing Company. 5th Aug. 2014.

[151] Damir Poles, Angela Nuic and Vincent Mouillet. 'Advanced aircraft performance modeling for ATM: Analysis of BADA model capabilities'. In: The 29th IEEE/AIAA Digital Avionics Systems Conference. Salt Lake City (Utah), USA, Oct. 2010, pp. 1.D.1-1-14. DOI: 10.1109/DASC. 2010. 5655518.

[152] Stuart J. Russell and Peter Norvig. Artificial Intelligence: A Modern Approach. Third Edition. Pearson, Dec. 2009. 1153 pp. ISBN: 978-0-13604259-4.

[153] Singiresu S. Rao. Engineering Optimization: Theory and Practice. 4th Edition. USA: John Wiley \& Sons, 2009. 835 pp. ISBN: 978-0-470-183526.

[154] Laurence J. Clancy. Aerodynamics. Pitman Aeronautical Engineering Series. London, UK: Pitman, 1975. 610 pp. ISBN: 978-0-470-15837-1.

[155] Michael L. Fredman and Robert Endre Tarjan. 'Fibonacci Heaps and Their Uses in Improved Network Optimization Algorithms'. In: Journal of the ACM 34.3 (July 1987), pp. 596-615. ISSN: 0004-5411. DOI: 10 . $1145 / 28869.28874$.

[156] Robert Fourer, David M. Gay and Brian W. Kernighan. AMPL: A Modeling Language for Mathematical Programming. Second Edition. Pacific Grove (California), USA: Cengage Learning, Nov. 2002. 540 pp. ISBN: 978-0534388096. URL: http://ampl . com/resources/the-ampl-book/.

[157] International Business Machines Corp. CPLEX Optimizer. 2015. URL: http://www-01.ibm.com/software/commerce/optimization/cplexoptimizer/index.html.

[158] Richard Alligier, David Gianazza and Nicolas Durand. 'Energy rate prediction using an equivalent thrust setting profile'. In: 5th International Conference on Research in Air Transportation. Berkeley (California), USA, May 2012, p. 8. URL: https://hal-enac archives-ouvertes . $\mathrm{fr} / \mathrm{hal}-00938734 /$ document.

[159] Mohan Ganesalingam. The Language of Mathematics: A Linguistic and Philosophical Investigation. Vol. 7805. Lecture Notes in Computer Science. Berlin, Heidelberg: Springer Berlin Heidelberg, 2013. ISBN: 978-3642-37011-3. DOI: $10.1007 / 978-3-642-37012-0$. 


\section{Part V}

\section{Appendices}





\section{Appendix A}

\section{AIDL for commercial fixed-wing aircraft}

The fixed-wing AIDL (FW-AIDL) is a formal language based on the abstraction of the key guidance inputs available for commanding an aircraft [47]. This language results from the analysis of the equations of motion of commercial fixedwing aircraft $[15,16,48]$. This analysis shows the three control input needed to compute an aircraft trajectory: bank angle, lift, and throttle. Additionally, in fixed-wing aircraft, there are typically three aerodynamic configuration means affecting aircraft performance: high lift devices, speed brakes, and landing gear position. The combined control and configuration input can be considered the six degrees of freedom (DoFs) of the aircraft motion model (AMM). Either the action of the pilot or the flight control system (FCS) must close these DoFs at any instant.

Computing an aircraft trajectory can be considered a constrained mechanical problem where each of the DoFs can be closed by a constraint. The combination of these mathematical constraints can be used to close the six DoFs, thus completely defining the aircraft's motion [16]. The application of the theory of differential algebraic equations (DAEs) to this constrained mechanical problem results in the mathematical structure of these constraints and the rules to combine them.

A complete description of this language can be found in [16]. A summary of the language's elements and features is provided next ${ }^{1}$.

\section{A.1 Language description}

The FW-AIDL provides a set of instructions - where each instruction defines a constraint to the aircraft motion - and a series of rules that govern how instructions can be combined to close the six DoFs.

${ }^{1}$ This summary is a modification of the description in [128] 


\begin{tabular}{ll|ll} 
Code & Instruction & Code & Instruction \\
\hline \hline ST & set throttle & HSL & horizontal speed law \\
TL & throttle law & HHS & hold horizontal speed \\
HT & hold throttle & VSL & vertical speed law \\
OLT & open loop throttle & HVS & hold vertical speed \\
SBA & set bank angle & TLP & track lateral path \\
BAL & bank angle law & TVP & track vertical path \\
HBA & hold bank angle & SHL & set high lift \\
OLBA & open loop bank angle & HLL & high lift law \\
SL & speed law & HHL & hold high lift \\
HS & hold speed & SSB & set speed brakes \\
SPA & set path angle & SBL & speed brakes law \\
PAL & path angle law & HSB & hold speed brakes \\
HPA & hold path angle & OLSB & open loop speed brakes \\
OLPA & open loop path angle & SLG & set landing gear \\
CL & course law & LGL & landing gear law \\
HC & hold course & HLG & hold landing gear \\
AL & altitude law & EL & energy law \\
HA & hold altitude & HE & hold energy
\end{tabular}

Table A.1: AIDL instructions for fixed-wing aircraft.

\section{A.1.1 Instructions}

AIDL instructions define the constraints used to close the six DoFs of aircraft motion. They are characterized by their effect, i.e., how the aircraft motion is affected by the instruction; and their execution interval, i.e., the time interval aircraft motion is affected by the instruction. Each instruction closes exactly one DoF of aircraft motion. The complete list of instruction effects available in FW-AIDL is shown in table A.1.

Instructions may be classified by their effect into five categories:

1. Set instructions model the change of a parameter of motion or configuration towards a target value governed by the Aircraft Model (e.g., landing gear deployment).

2. Law instructions serve to control any given parameter as a function of dynamic state, enabling advanced guidance modes based on airborne sensor measures (e.g., a law controlling the Mach number as a function of altitude).

3. Hold instructions serve to maintain a specific variable constant, independently of its value (e.g., hold altitude).

4. Open Loop instructions model the direct action of the pilot or FMS over the available controls (e.g., using the throttle lever), without feedback from the aircraft state.

5. Track instructions can be used to guide an aircraft along a geometric path (e.g., follow a geodesic curve). They allow the definition of implicit 


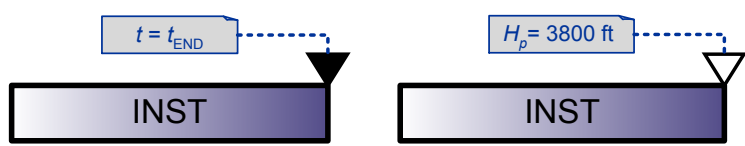

(a) Fixed trigger.

(b) Floating trigger.

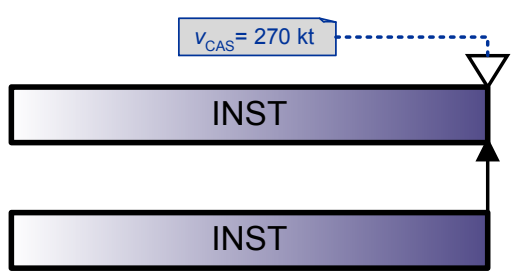

(c) Linked trigger.

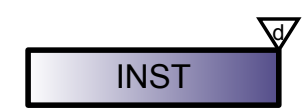

(d) Default trigger.

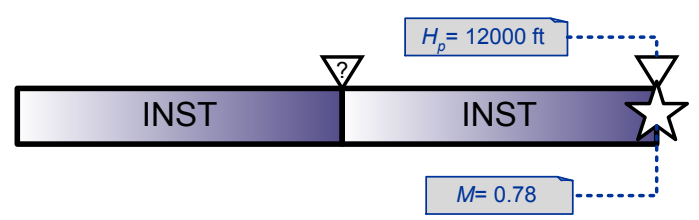

(e) Auto trigger.

Figure A.1: Graphical representation of AIDL triggers.

relations between dynamic state variables, equivalent to laws if written in an explicit form.

Many instructions require some additional parameters to completely define the effect. These are called AIDL instruction parameters, and may contain a magnitude (a physical value with units), a function (a description of a mathematical function), or a specifier (a label of a predefined set of values). For instance, the hold speed effect requires a specifier indicating the type of speed to be held: Mach number, true airspeed (TAS), calibrated airspeed (CAS), or indicated airspeed (IAS). Other instructions require different parameters, such as the set path angle instruction, which requires a magnitude indicating the target path angle and a specifier indicating whether it is a geometric (GEO) or aerodynamic (AER) path angle. Meanwhile, the altitude law instruction requires a specifier indicating the type of altitude - either geometric (GEO) or pressure (PRE) - and a function defining the altitude during the instruction's execution interval.

\section{Triggers}

An execution interval is defined by an end condition called AIDL trigger. The AIDL has five types of triggers:

1. Fixed triggers consist of a time or distance limit, independent of aircraft motion state. An instruction with a fixed trigger at time $t=t_{\mathrm{END}}$ is shown in figure A.1a. 


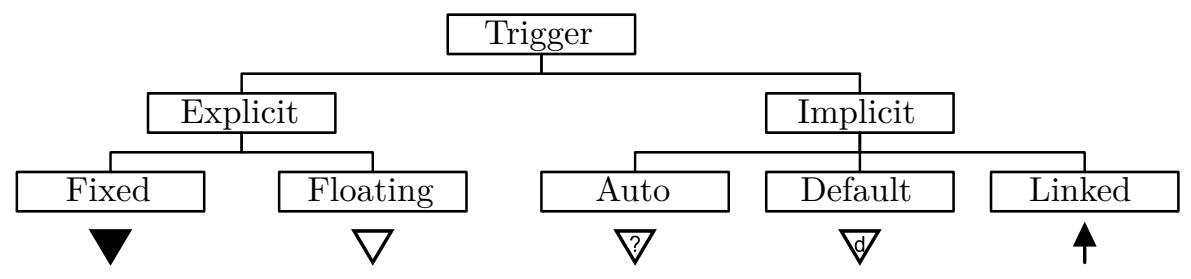

Figure A.2: Hierarchy of triggers.

2. Floating triggers consist of a mathematical condition involving motion states or configuration variables. An instruction with a floating trigger (pressure altitude equal to 3800 feet) is shown in figure A.1b.

3. Linked triggers represent the situation where an instruction ends synchronously to other AIDL instruction. Parallel instructions ending simultaneously (using a linked trigger and a floating trigger with CAS of 270 knots) are shown in figure A.1c.

4. Default triggers are always associated to set instructions, and indicate the time at which the demanded change in motion or configuration is completed. A set instruction with a default end trigger is shown in figure A.1d.

5. Auto triggers are used to satisfy future conditions over the aircraft state. They make instructions end so that a condition over dynamic state is fulfilled in a later time corresponding to another trigger. Any intent including auto triggers needs to be resolved by iteration of the integration process or by backwards integration. Potential applications of auto triggers would be the definition of top of descent (TOD) in continuous descent approaches (CDAs), or required time of arrival (RTA) constraint implementation. Figure A.1e shows two consecutive instructions (from left to right), the first ending with an auto trigger; and the second, with a floating trigger with barometric altitude of 12000 feet. A condition requiring a Mach of 0.78 at the time of the second trigger is used to close the auto trigger.

These triggers can be classified as explicit and implicit, as shown in figure A.2. Explicit triggers comprise the fixed and floating triggers, whereas implicit triggers comprise linked, default, and auto triggers [48].

\section{A.1.2 Intent}

This section briefly describes the rules necessary to produce correct aircraft intent in FW-AIDL, i.e., a FW-AIDL word. A FW-AIDL word corresponds to a specific guidance strategy during a time interval. Therefore, six parallel lists of instructions will compose an FW-AIDL word, closing the six DoFs of the aircraft motion at any time. To achieve this, the FW-AIDL instructions are ordered in six parallel threads. From the mathematical analysis [15, 16], two FW-AIDL threads (longitudinal threads) can include instruction sequences that define aspects of the aircraft speed, altitude, engine setting, or energy. Another FW-AIDL thread (lateral thread) can contain instructions defining the 


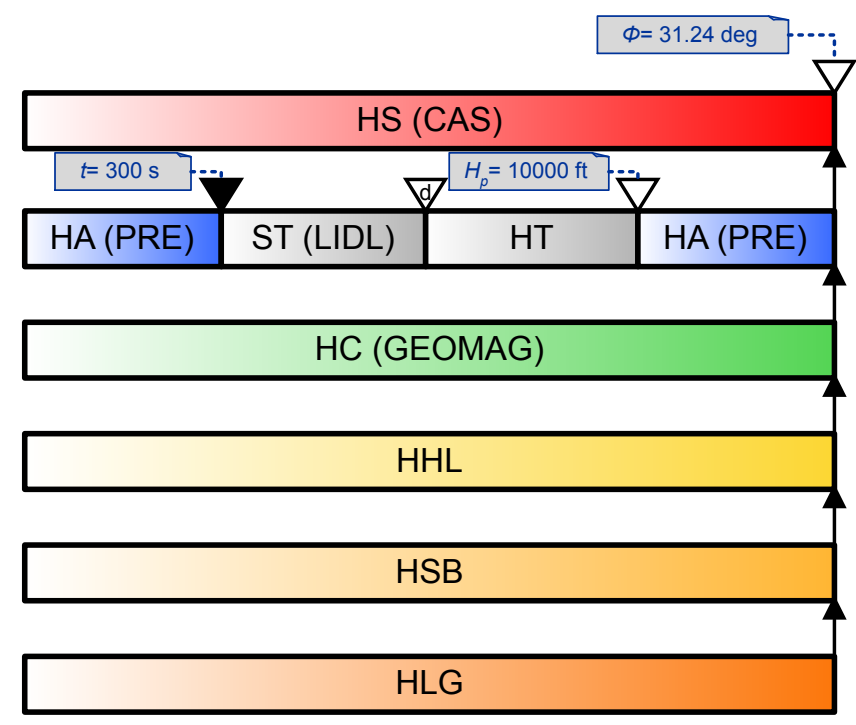

Figure A.3: Example AIDL word ending with a master trigger.

\begin{tabular}{l|l|l} 
Profile & Instructions & Thread \\
\hline \hline Speed & SL, HS, HSL, HHS, VSL, HVS, EL, & \multirow{2}{*}{$\begin{array}{l}\text { Longitudinal } \\
\text { (1st or 2nd) }\end{array}$} \\
\hline Vertical & AL, HA, VSL, HVS, PAL, HPA, & \\
& TVP & \\
\hline Propulsive & ST, TL, HT, OLT & \\
\hline Lateral & $\begin{array}{l}\text { SBA, BAL, HBA, OLBA, CL, HC, }, \\
\text { TLP }\end{array}$ & Lateral \\
\hline High lift & SHL, HLL, HHL & High lift \\
\hline Speed brakes & SSB, SBL, HSB, OLSB & Speed brakes \\
\hline Landing gear & SLG, LGL, HLG & Landing gear
\end{tabular}

Table A.2: Instruction profiles and related threads.

lateral aspects of the aircraft motion, such as geometric path, heading, bearing or bank angle. The remaining three threads include instructions defining the configuration: high lift devices thread, speed brakes thread, and landing gear thread.

Each thread consists on a sequence of one or more consecutive time-ordered FW-AIDL instructions. The start of every instruction is assumed to be the end of the previous instruction in the same thread (except for the first instruction of the thread, corresponding to the flight's initial conditions (ICs)). The six execution threads start and end simultaneously. In order to guarantee that all execution threads end at the same time, all the final instructions of each thread but one must finish in linked triggers. They must point to the remaining one, known as the master trigger, as in the example in figure A.3.

Not every instruction type can be placed in each thread; they have been grouped into seven different profiles, as outlined in table A.2. For the 1st and 
2nd thread there is an additional rule: at any time it must be ensured that only one instruction of the speed, vertical or propulsive types is active. 


\section{Appendix B}

\section{Dynamic model of a quadrotor}

A quadrotor is a rotary-wing aircraft capable of vertical, stationary, and lateral flight. It has four independent propellers arranged at the corners of a cross frame. Two propellers, attached at opposite corners of the frame, spin clockwise, whereas the other two spin counterclockwise. Quadrotors are controlled by commanding the rotation speed of each propeller. All propellers rotate at the same speed for stationary flight. By increasing or decreasing the speed of the four propellers together, vertical motion is generated. Pitch and roll rotation is produced by changing the speeds of two oppositely placed propellers conversely. Pitch and roll rotation combined can be used to tilt the quadrotor towards any orientation, thus producing lateral movement towards such orientation. To produce yaw, the counter-torque produced by the two clockwise and two counterclockwise propellers must be different.

Modeling the dynamics of a system is a necessary step in developing the control of such system. As part of quadrotor controller research, several authors have modeled the dynamics of a quadrotor using different methods [133-138]. The two dominating methods are the Euler-Lagrange formalism and the NewtonEuler formalism [139]. In this thesis, the model proposed in [140] is used as the starting point, which was modeled applying the Newton-Euler formalism. This model does not take into account some aerodynamic effects such as rolling moment (also called blade flapping in the literature), ground and ceiling effects, hub forces, or friction [139]; and it was later improved to include most of these effects $[133,134]$. However, this earlier model — with the addition of friction forces - is detailed enough for the needs of this thesis.

This appendix presents the dynamic model used in this thesis. Figure B.1 shows a quadrotor as described before, numbering each propeller and specifying its direction of rotation. The earth-fixed frame $E$ and body-fixed frame $B$ that will be used as reference also appear in this figure. The orientation of $B$ with respect to $E$ can be described using Tait-Bryan angles [134]. These angles represent three successive rotations about the axes of the body-fixed frame, and they are called roll $(\phi)$, pitch $(\theta)$, and yaw $(\psi)$. The rotation corresponding to 


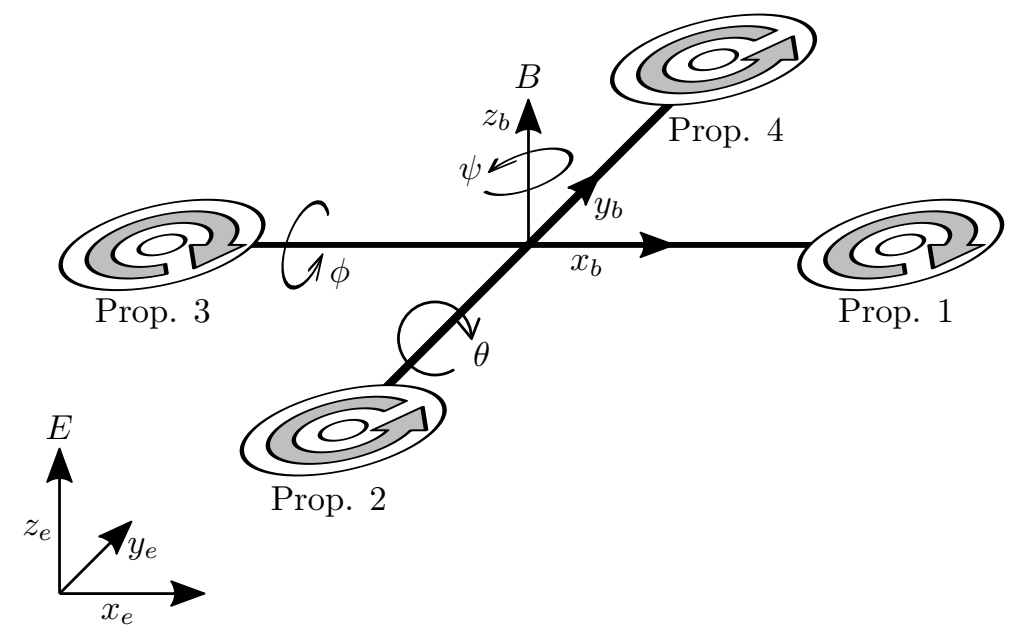

Figure B.1: Configuration and reference frames of a quadrotor.

each angle can be expressed as a rotation matrix $\mathbf{R}_{\phi}, \mathbf{R}_{\theta}, \mathbf{R}_{\psi} \in S O(3)^{1}$.

$$
\begin{aligned}
& \mathbf{R}_{\phi}= {\left[\begin{array}{ccc}
1 & 0 & 0 \\
0 & \cos \phi & -\sin \phi \\
0 & \sin \phi & \cos \phi
\end{array}\right] } \\
& \mathbf{R}_{\theta}= {\left[\begin{array}{ccc}
\cos \theta & 0 & \sin \theta \\
0 & 1 & 0 \\
-\sin \theta & 0 & \cos \theta
\end{array}\right] } \\
& \mathbf{R}_{\psi}=\left[\begin{array}{ccc}
\cos \phi & -\sin \phi & 0 \\
\sin \phi & \cos \phi & 0 \\
0 & 0 & 1
\end{array}\right]
\end{aligned}
$$

The multiplication of (B.1), (B.2), and (B.3) results in the rotation matrix $\mathbf{R}$, which transforms the orientation from frame $B$ to $E$.

$$
\begin{aligned}
\mathbf{R} & =\mathbf{R}_{\psi} \mathbf{R}_{\theta} \mathbf{R}_{\phi} \\
& =\left[\begin{array}{ccc}
\cos \theta \cos \psi & \sin \phi \sin \theta \cos \psi-\cos \phi \sin \psi & \cos \phi \sin \theta \cos \psi+\sin \phi \sin \psi \\
\cos \theta \sin \psi & \sin \phi \sin \theta \sin \psi+\cos \phi \cos \psi & \cos \phi \sin \theta \sin \psi-\sin \phi \cos \psi \\
-\sin \theta & \sin \phi \cos \theta & \cos \phi \cos \theta
\end{array}\right]
\end{aligned}
$$

This model was derived under the following assumptions [134]:

- The origin of the body-fixed frame $B$ and the center of mass of the quadrotor coincide.

- The structure of the quadrotor is rigid and symmetrical.

${ }^{1} S O(n)=\left\{\mathbf{R} \in \mathbb{R}_{n \times n} \mid \mathbf{R} \mathbf{R}^{\top}=\mathbf{R}^{\top} \mathbf{R}=\mathbf{I}_{n}\right\}$ is the rotation group in dimension $n$. 
- The propellers are rigid.

- Thrust and drag are proportional to the square of propeller's speed.

- Friction is proportional to the quadrotor's speed.

- Mass of the quadrotor is constant.

\section{B.1 Physical effects}

The physical effects that act on the quadrotor according to this model can be grouped into two categories, depending on whether they affect the position or the orientation of the aircraft.

The position of the quadrotor is affected by:

- Propeller thrust. Each propeller produces an aerodynamic force proportional to the square of its rotation speed. These forces act in the direction of the positive $z_{b}$ axis (upward in frame $B$ ).

$$
\mathbf{R}\left[\begin{array}{l}
0 \\
0 \\
1
\end{array}\right] U_{1}
$$

where $\mathbf{R}$ transforms the orientation from frame $B$ to $E$,

$$
U_{1}=b\left(\Omega_{1}^{2}+\Omega_{2}^{2}+\Omega_{3}^{2}+\Omega_{4}^{2}\right)
$$

is the sum of the forces of all propellers, $\Omega_{i}$ is the rotation speed of propeller $i$, and $b$ is the thrust coefficient.

- Weight. This force is proportional to the mass of the quadrotor, and acts in the direction of the negative $z_{e}$ axis (downward in frame $E$ ).

$$
-m g\left[\begin{array}{l}
0 \\
0 \\
1
\end{array}\right]
$$

where $m$ is the mass of the quadrotor, $g$ is the acceleration due to gravity.

- Friction. This force is proportional to the aircraft's velocity with respect to the wind, and acts in the direction opposite to the movement [135, 141].

$$
-\mathbf{R}\left[\begin{array}{ccc}
k_{s} & 0 & 0 \\
0 & k_{s} & 0 \\
0 & 0 & k_{u}
\end{array}\right] \mathbf{R}^{\top}(\mathbf{v}-\mathbf{w})
$$

where $k_{s}$ and $k_{u}$ are the friction coefficients for the horizontal and vertical movement, respectively; and $\mathbf{v}$ and $\mathbf{w}$ are, respectively, the aircraft's 
velocity vector and the wind speed vector in frame $E$, described in (B.9) and (B.10).

$$
\begin{array}{r}
\mathbf{v}=\left[\begin{array}{l}
\dot{x_{e}} \\
\dot{y}_{e} \\
\dot{z_{e}}
\end{array}\right] \\
\mathbf{w}=\left[\begin{array}{l}
w_{x} \\
w_{y} \\
w_{z}
\end{array}\right]
\end{array}
$$

To simplify expression (B.8), it is assumed that values of $\phi$ and $\theta$ are sufficiently small to make the following approximation:

$$
\mathbf{R}\left[\begin{array}{ccc}
k_{s} & 0 & 0 \\
0 & k_{s} & 0 \\
0 & 0 & k_{u}
\end{array}\right] \mathbf{R}^{\top} \approx\left[\begin{array}{ccc}
k_{s} & 0 & 0 \\
0 & k_{s} & 0 \\
0 & 0 & k_{u}
\end{array}\right]
$$

The orientation of the quadrotor is affected by:

- Propeller action. This includes the rolling and pitching torques, which are caused by propellers at opposite sides of the frame spinning with different speeds; and the yawing counter-torque unbalance, caused by the difference of counter-torques at each propeller.

$$
\left[\begin{array}{c}
l U_{2} \\
l U_{3} \\
U_{4}
\end{array}\right]
$$

with

$$
\begin{aligned}
& U_{2}=b\left(\Omega_{4}^{2}-\Omega_{2}^{2}\right) \\
& U_{3}=b\left(\Omega_{3}^{2}-\Omega_{1}^{2}\right) \\
& U_{4}=d\left(\Omega_{2}^{2}+\Omega_{4}^{2}-\Omega_{1}^{2}-\Omega_{3}^{2}\right)
\end{aligned}
$$

where $l$ is the distance between the propeller and the center of mass, $b$ is the thrust coefficient, and $d$ is the drag coefficient.

- Body gyroscopic effect. This gyroscopic torque results from the rotation of the quadrotor.

$$
-\hat{\omega} \mathbf{J} \boldsymbol{\omega}
$$

where

$$
\boldsymbol{\omega}=\left[\begin{array}{c}
\dot{\phi} \\
\dot{\theta} \\
\dot{\psi}
\end{array}\right]
$$

is the rotation speed vector,

$$
\hat{\boldsymbol{\omega}}=\left[\begin{array}{ccc}
0 & -\dot{\psi} & \dot{\theta} \\
\dot{\psi} & 0 & -\dot{\phi} \\
-\dot{\theta} & \dot{\phi} & 0
\end{array}\right]
$$


is the skew-symmetric matrix such that $\hat{\boldsymbol{\omega}} \mathbf{q}=\boldsymbol{\omega} \times \mathbf{q}$ for any $\mathbf{q} \in \mathbb{R}^{3}$, and

$$
\mathbf{J}=\left[\begin{array}{ccc}
I_{x} & 0 & 0 \\
0 & I_{y} & 0 \\
0 & 0 & I_{z}
\end{array}\right]
$$

is the moment of inertia tensor. Since the origin of $B$ is at the center of mass, $\mathbf{J}$ is a diagonal matrix, where the moments of inertia along the $x_{b}$, $y_{b}$, and $z_{b}$ axes are $I_{x}, I_{y}$, and $I_{z}$, respectively.

- Propeller gyroscopic effect. Changes in the roll or pitch of the aircraft force a change in the angular momentum of the four propellers. This imposes a gyroscopic torque to the aircraft that can be mathematically described as:

$$
-J_{r}\left[\begin{array}{c}
\dot{\theta} \Omega \\
\dot{\phi} \Omega \\
0
\end{array}\right]
$$

where $J_{r}$ is the rotor inertia, and

$$
\Omega=\Omega_{2}+\Omega_{4}-\Omega_{1}-\Omega_{3}
$$

is the sum of all angular rates of the rotors.

- Friction torque. This torque is proportional to the rotation speed but in opposite direction [135].

$$
-k_{r} \boldsymbol{\omega}
$$

where $k_{r}$ is the frictional coefficient due to rotational motion, and $\omega$ is the rotation speed vector defined in (B.16).

\section{B.2 Equations of motion}

All the forces and moments in section B.1 can be combined to obtain the equations of motion of the quadrotor. Equation (B.22) results from combining (B.5), (B.7), and (B.8); while equation (B.23) results from combining (B.11), (B.15), (B.19), and (B.21).

$$
\begin{aligned}
& m \dot{\mathbf{v}}=\mathbf{R}\left[\begin{array}{l}
0 \\
0 \\
1
\end{array}\right] U_{1}-m g\left[\begin{array}{l}
0 \\
0 \\
1
\end{array}\right]-\left[\begin{array}{ccc}
k_{s} & 0 & 0 \\
0 & k_{s} & 0 \\
0 & 0 & k_{u}
\end{array}\right](\mathbf{v}-\mathbf{w}) \\
& \mathbf{J} \dot{\boldsymbol{\omega}}=\left[\begin{array}{c}
l U_{2} \\
l U_{3} \\
U_{4}
\end{array}\right]-\hat{\boldsymbol{\omega}} \mathbf{J} \boldsymbol{\omega}-J_{r}\left[\begin{array}{c}
\dot{\theta} \Omega \\
\dot{\phi} \Omega \\
0
\end{array}\right]-k_{r} \boldsymbol{\omega}
\end{aligned}
$$

The range of flight of a quadrotor is usually limited to a small area where a flat-Earth coordinate system (Cartesian system) can be used. Therefore, the aircraft's position $\boldsymbol{\zeta}$ and orientation $\boldsymbol{\eta}$ can be obtained from:

$$
\begin{gathered}
\dot{\zeta}=\mathbf{v} \\
\dot{\eta}=\omega
\end{gathered}
$$


where

$$
\begin{aligned}
\boldsymbol{\zeta} & =\left[\begin{array}{l}
x_{e} \\
y_{e} \\
z_{e}
\end{array}\right] \\
\boldsymbol{\eta} & =\left[\begin{array}{l}
\phi \\
\theta \\
\psi
\end{array}\right]
\end{aligned}
$$

Equations (B.22) and (B.23) can be written as a set of implicit ordinary differential equations (ODEs):

$$
\begin{aligned}
m \ddot{x}_{e} & =(\cos \phi \sin \theta \cos \psi+\sin \phi \sin \psi) U_{1}-k_{s}\left(\dot{x}_{e}-w_{x}\right) \\
m \ddot{y}_{e} & =(\cos \phi \sin \theta \sin \psi-\sin \phi \cos \psi) U_{1}-k_{s}\left(\dot{y}_{e}-w_{y}\right) \\
m \ddot{z}_{e} & =(\cos \phi \cos \theta) U_{1}-m g-k_{u}\left(\dot{z}_{e}-w_{z}\right) \\
I_{x} \ddot{\phi} & =l U_{2}-\dot{\theta} \dot{\psi}\left(I_{z}-I_{y}\right)-J_{r} \dot{\theta} \Omega-k_{r} \dot{\phi} \\
I_{y} \ddot{\theta} & =l U_{3}-\dot{\phi} \dot{\psi}\left(I_{z}-I_{y}\right)-J_{r} \dot{\phi} \Omega-k_{r} \dot{\theta} \\
I_{z} \ddot{\psi} & =U_{4}-\dot{\phi} \dot{\theta}\left(I_{z}-I_{y}\right)-k_{r} \dot{\psi}
\end{aligned}
$$

These equations can be trivially transformed into explicit ODEs.

\section{B.3 Quadrotor parameters}

The equations of motion derived in B.2 make use of several parameters that are aircraft dependent. In order for the equations to be complete, these parameters must be provided. This section provides the aerodynamic parameters corresponding to three different aircraft: the omnidirectional stationary flying outstretched robot (OS4) [134], the Cornell autonomous flying vehicle (AFV) [135], and the Australian National University (ANU) X4-Flyer Mark III [142]. Other sets of parameters appear in the literature [136, 138], but there is no claim that those parameters correspond to real aircraft.

Table B.1 shows the aforementioned aerodynamic parameters. Note only one of the references provides the frictional coefficients. 


\begin{tabular}{r|c|c|c|c} 
Parameter & OS4 & AFV & X4-Flyer & Units \\
\hline \hline$m$ & 0.65 & 4.493 & 4.34 & $\mathrm{~kg}$ \\
$I_{x}$ & $7.5 \cdot 10^{-3}$ & 0.177 & 0.0820 & $\mathrm{~kg} \mathrm{~m}^{2}$ \\
$I_{y}$ & $7.5 \cdot 10^{-3}$ & 0.177 & 0.0845 & $\mathrm{~kg} \mathrm{~m}^{2}$ \\
$I_{z}$ & $1.3 \cdot 10^{-2}$ & 0.334 & 0.1377 & $\mathrm{~kg} \mathrm{~m}^{2}$ \\
$b$ & $3.13 \cdot 10^{-5}$ & $8.49 \cdot 10^{-5}$ & $1.295 \cdot 10^{-5}$ & $\mathrm{~N} \mathrm{~s}^{2}$ \\
$d$ & $7.5 \cdot 10^{-7}$ & $3.15 \cdot 10^{-6}$ & $1.0368 \cdot 10^{-7}$ & $\mathrm{~N} \mathrm{~m} \mathrm{~s}^{2}$ \\
$J_{r}$ & $6 \cdot 10^{-5}$ & $1.46 \cdot 10^{-3}$ & $4.0887 \cdot 10^{-5}$ & $\mathrm{~kg} \mathrm{~m}^{2}$ \\
$l$ & 0.23 & 0.38 & 0.315 & $\mathrm{~m}$ \\
$k_{s}$ & $\mathrm{n} / \mathrm{a}$ & 1 & $\mathrm{n} / \mathrm{a}$ & $\mathrm{N} \mathrm{s} \mathrm{m}^{-1}$ \\
$k_{u}$ & $\mathrm{n} / \mathrm{a}$ & 1 & $\mathrm{n} / \mathrm{a}$ & $\mathrm{N} \mathrm{s} \mathrm{m}^{-1}$ \\
$k_{r}$ & $\mathrm{n} / \mathrm{a}$ & 0.35 & $\mathrm{n} / \mathrm{a}$ & $\mathrm{N} \mathrm{m} \mathrm{sad}^{-1}$
\end{tabular}

Table B.1: Quadrotor parameters 



\section{Appendix C}

\section{Basic ICDL templates}

This annex describes in detail the templates used along the thesis.

\section{C.1 Commercial flight templates}

Descriptions of ICDL templates applicable to commercial flights are provided in this section. Many of these descriptions have been extracted from [128]. For a complete reference of the AIDL instructions and its parameters (magnitudes and specifiers), refer to [16].

\section{C.1.1 Vertical profile}

\section{Level flight}

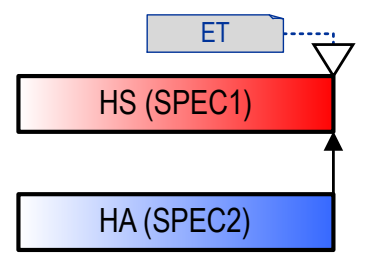

Figure C.1: 'Level flight' template.

This template maintains speed and altitude, as shown in figure C.1. A composite created from this template is denoted 'Level flight $\langle\mathrm{SPEC} 1, \mathrm{SPEC} 2, \mathrm{ET}\rangle$ ', where the undefined parameters are:

1. SPEC1: a set of AIDL specifiers indicating the possible types of speed (CAS, TAS, IAS, Mach, etc.).

2. SPEC2: a set of AIDL specifiers indicating the possible types of altitude, geometric altitude (GEO) or geopotential pressure altitude (PRE).

3. ET: the end trigger of the composite. 


\section{Ascent/descent}

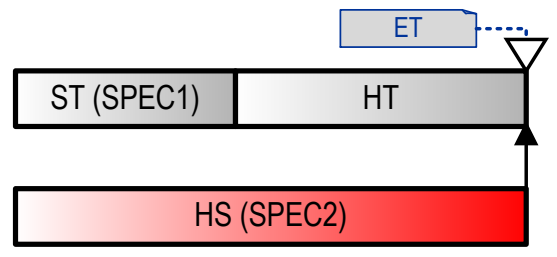

Figure C.2: 'Ascent/descent' template.

This template represents an ascent or descent manoeuvre with constant speed, as shown in C.2. A composite created from this template is denoted 'Ascent/descent $\langle\mathrm{SPEC} 1, \mathrm{SPEC} 2, \mathrm{ET}\rangle$ ', where the undefined parameters are:

1. SPEC1: a set of AIDL specifiers indicating the possible types of thrust regime. Low idle regime (LIDL) is commonly used for descent, and maximum climb regime (MCMB) for ascent.

2. SPEC2: a set of AIDL specifiers indicating the possible types of speed (CAS, TAS, IAS, Mach, etc.).

3. ET: the end trigger of the composite.

\section{Level thrust acceleration/deceleration}

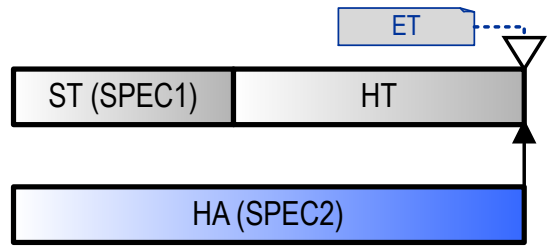

Figure C.3: 'Level thrust acceleration/deceleration' template.

This template represents a leveled acceleration or deceleration, as shown in figure C.3. A composite created from this template is denoted 'Level thrust acceleration/deceleration $\langle\mathrm{SPEC} 1, \mathrm{SPEC} 2, \mathrm{ET}\rangle$ ', where the undefined parameters are:

1. SPEC1: a set of AIDL specifiers indicating the possible types of thrust regime. Low idle regime (LIDL) is commonly used for deceleration, and maximum cruise regime (MCRZ) for acceleration.

2. SPEC2: a set of AIDL specifiers indicating the possible types of altitude, geometric altitude (GEO) or geopotential pressure altitude (PRE).

3. ET: the end trigger of the composite. 


\section{CAS/Mach climb}

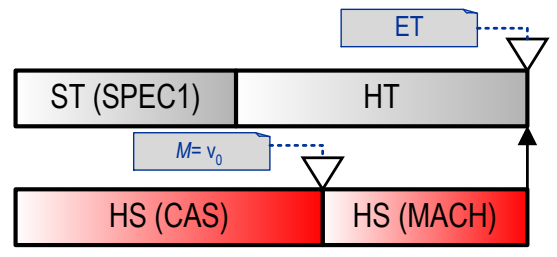

Figure C.4: 'CAS/Mach climb' template.

This template represents an ascent manoeuvre with constant CAS until the crossover altitude, then with constant Mach, as shown in C.4. A composite created from this template is denoted 'CAS/Mach climb $\left\langle\mathrm{SPEC} 1, v_{0}, \mathrm{ET}\right\rangle$ ', where the undefined parameters are:

1. SPEC1: a set of AIDL specifiers indicating the possible types of thrust regime. Maximum climb regime $(\mathrm{MCMB})$ is the most common specifier for this composite.

2. $v_{0}$ : an interval of possible Mach speeds to be flown in the second segment of the composite, after the crossover altitude.

3. ET: the end trigger of the composite.

\section{Mach/CAS descent}

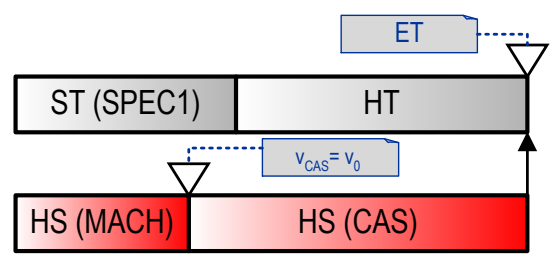

Figure C.5: 'Mach/CAS descent' template.

This template represents a descent manoeuvre with constant Mach until the crossover altitude, then with constant CAS, as shown in C.5. A composite created from this template is denoted 'Mach/CAS descent $\left\langle\mathrm{SPEC1,} v_{0}, \mathrm{ET}\right\rangle$ ', where the undefined parameters are:

1. SPEC1: a set of AIDL specifiers indicating the possible types of thrust regime. Low idle regime (LIDL) is the most common specifier for this composite.

2. $v_{0}$ : an interval of possible CAS to be flown in the second segment of the composite, after the crossover altitude.

3. ET: the end trigger of the composite. 


\section{Constant path ascent/descent}

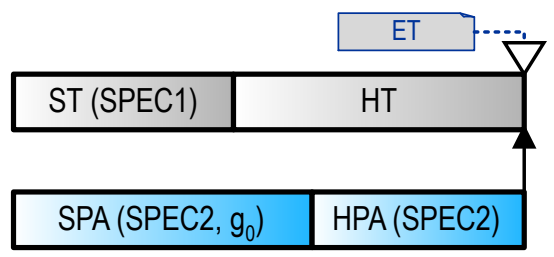

Figure C.6: 'Constant path ascent/descent' template.

This template represents a ascent or descent manoeuvre, where the path angle is maintained constant, as shown in figure C.6. A composite created from this

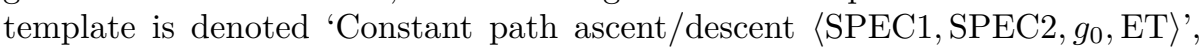
where the undefined parameters are:

1. SPEC1: a set of AIDL specifiers indicating the possible types of thrust regime. Low idle regime (LIDL) is commonly used for descent, and maximum climb regime (MCMB) for ascent.

2. SPEC2: a set of AIDL specifiers indicating the possible types of path angle, geometric path angle (GEO) or aerodynamic path angle (AER).

3. $g_{0}$ : an interval of possible path angle magnitudes (e.g., 3 deg : 3..4). Positive values for ascent, negative values for descent.

4. ET: the end trigger of the composite.

\section{Constant path constant speed ascent/descent}

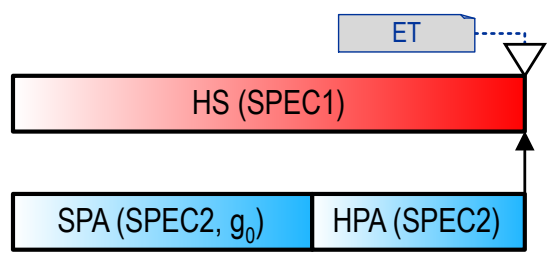

Figure C.7: 'Constant path constant speed ascent/descent' template.

This template represent an ascent or descent manoeuvre, where the path angle and speed are maintained constant, as shown in figure C.7. A composite created from this template is denoted 'Constant path constant speed ascent/ descent $\left\langle\mathrm{SPEC} 1, \mathrm{SPEC} 2, g_{0}, \mathrm{ET}\right\rangle$ ', where the undefined parameters are:

1. SPEC1: a set of AIDL specifiers indicating the possible types of altitude, geometric altitude (GEO) or geopotential pressure altitude (PRE).

2. SPEC2: a set of AIDL specifiers indicating the possible types of speed (CAS, TAS, IAS, Mach, etc.). 
3. $g_{0}$ : an interval of possible path angle magnitudes (e.g., $\left.-2 \mathrm{deg}:-4 . .-1.5\right)$. Positive values for ascent, negative values for descent.

4. ET: the end trigger of the composite.

\section{Ascent acceleration with ESF}

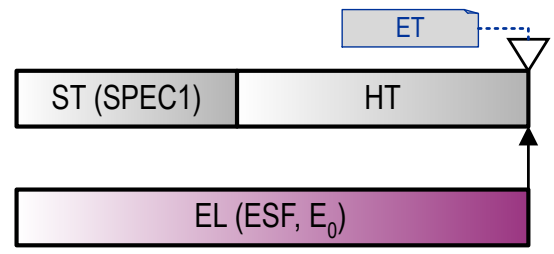

Figure C.8: 'Ascent acceleration with ESF' template.

This template represents an ascent acceleration manoeuvre with a constant energy share factor (ESF) (which rules the repartition of the specific power between the speed and the altitude [158]), as shown in C.4. A composite created from this template is denoted 'Ascent acceleration with ESF $\left\langle\mathrm{SPEC} 1, E_{0}, \mathrm{ET}\right\rangle$ ', where the undefined parameters are:

1. SPEC1: a set of AIDL specifiers indicating the possible types of thrust regime. Maximum take-off regime (MTKF) and maximum climb regime $(\mathrm{MCMB})$ are common specifiers for this manoeuvre.

2. $E_{0}$ : an interval of possible ESFs, where an ESF of 0 means all energy is invested in increasing speed, whereas an ESF of 1 means all energy is invested in increasing altitude.

3. ET: the end trigger of the composite.

\section{C.1.2 Configuration profile}

\section{Clean configuration}

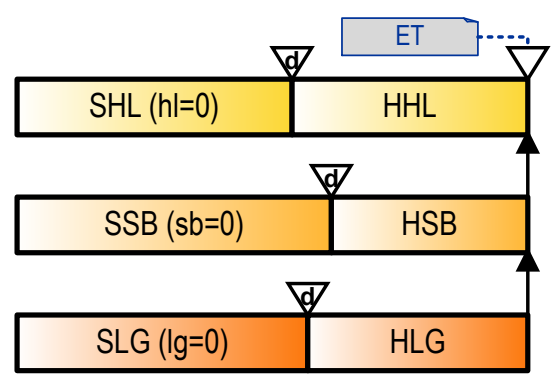

Figure C.9: 'Clean configuration' template.

This template represents a manoeuvre where high lift devices (flaps), speed breaks, and landing gear are kept retracted, as shown in figure C.9. A composite 
created from this template is denoted 'Clean configuration $\langle\mathrm{ET}\rangle$ ', where the undefined parameter is the end trigger of the composite, ET.

\section{Scheduled configuration}

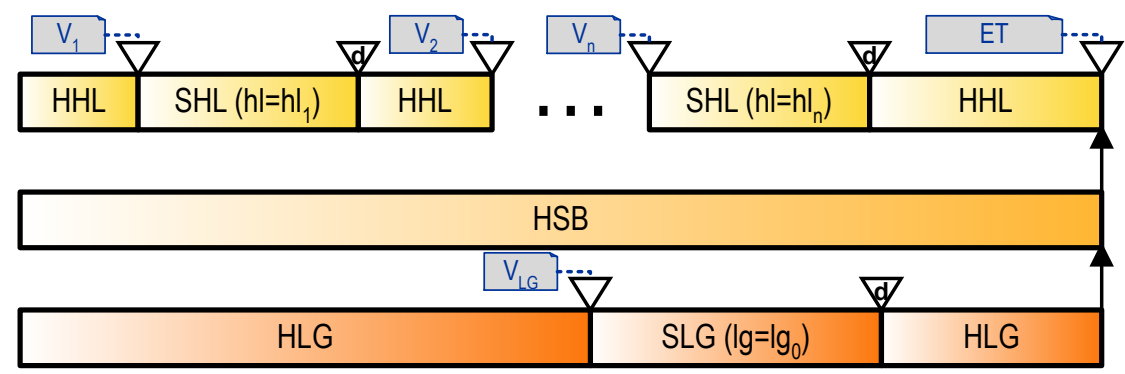

Figure C.10: 'Scheduled configuration' template.

This template represents a manoeuvre, during final approach or take-off, where the high lift devices (flaps) and landing gear configuration are modified to maintain a safe lift, as shown in figure C.10. A composite created from this template is denoted 'Scheduled configuration $\left\langle\mathrm{VLG}, l g_{0}, \mathrm{~V}, \mathrm{hl}, \mathrm{ET}\right\rangle$ ', where the undefined parameters are:

1. VLG: an interval of possible speed magnitudes triggering landing gear deployment or retraction.

2. $l g_{0}$ : a landing gear configuration magnitude. Set to 0 (retract) for take-off, and 1 (deploy) for the final approach.

3. V: list $\left\{\mathrm{V}_{1}, \ldots, \mathrm{V}_{n}\right\}$ of speeds triggering the next flap deflection.

4. hl: list $\left\{\mathrm{hl}_{1}, \ldots, \mathrm{hl}_{n}\right\}$ of target high lift configuration values after speed $\mathrm{V}_{i}$ is reached.

5. ET: the end trigger of the composite.

\section{C.1.3 Lateral profile}

\section{Geometric path waypoint}

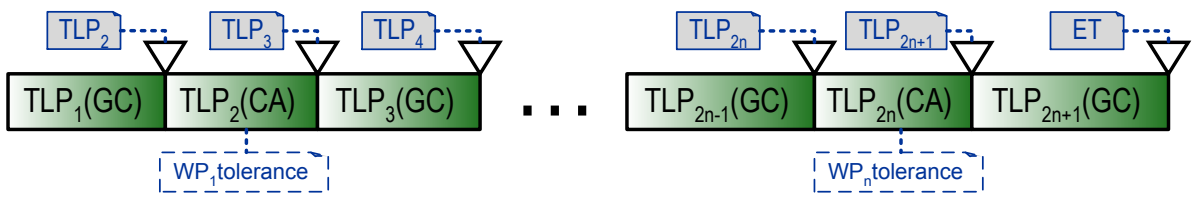

Figure C.11: 'Geometric path waypoint' template.

This template represents a manoeuvre where the aircraft flies a horizontal path defined by a sequence of waypoints. A total of $n+1$ waypoints are provided 
(a list with $n$ waypoints plus a waypoint trigger). The horizontal path consists on a sequence of great circles (GC) and circular arcs (CA), as shown in figure C.11. Great circles are connected using circular arcs in order to perform a fly-by manoeuvre at each point. The radius of each circular arc is computed from the waypoint's tolerance to ensure that the aircraft passes sufficiently close to the waypoint. A composite created from this template is denoted 'Geometric path waypoint $\langle\mathrm{WP}, \mathrm{ET}\rangle$ ', where the undefined parameters are:

1. WP: a list $\left\{\mathrm{WP}_{1}, \ldots, \mathrm{WP}_{n}\right\}$ of waypoints with their respective tolerances.

2. ET: the end trigger of the composite, which must be a waypoint trigger.

\section{C.2 Quadrotor templates}

The templates used in this thesis to build ICDL composites for quadrotor aircraft are included in this section. The instructions in which this templates are based correspond to the QR-AIDL, a variant of the AIDL designed to describe quadrotor trajectories.

\section{C.2.1 Lateral profile}

\section{Hover}

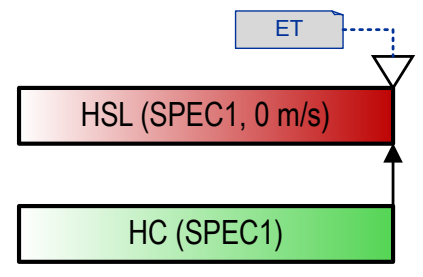

Figure C.12: 'Hover' template.

This template represents a hovering flight, where the quadrotor stays in the same horizontal position, as shown in figure C.12. A composite created from this template is denoted 'Hover $\langle\mathrm{SPEC} 1, \mathrm{ET}\rangle$ ', where the undefined parameters are:

1. SPEC1: a set of AIDL specifiers indicating the possible reference frames (EARTH or AIR).

2. ET: the end trigger of the composite. 


\section{Circle around}

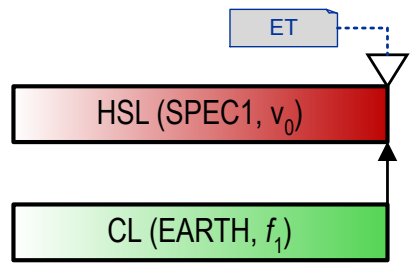

Figure C.13: 'Circle around' template.

This template represents a flight where the quadrotor flies in circles around a given point, as shown in figure C.13. A composite created from this template is denoted 'Circle around $\left\langle\mathrm{SPEC} 1, \mathrm{SPEC} 2, v_{0}, \mathrm{WP}, \mathrm{ET}\right\rangle$ ', where the undefined parameters are:

1. SPEC1: a set of AIDL specifiers indicating the possible reference frames (EARTH or AIR).

2. SPEC2: a set of AIDL specifiers indicating the direction of rotation, either clockwise $(\mathrm{CW})$ or counter-clockwise $(\mathrm{CCW})$.

3. $v_{0}$ : an interval of possible speeds (either horizontal speed $v_{h}$ or ground speed $v_{g}$, depending on the reference frame).

4. WP: a two-dimensional (2D) waypoint indicating the center of the rotation.

5. ET: the end trigger of the composite.

The function $f_{1}$ defines the geometric course $\chi_{e}$ as:

$$
f_{1}=\operatorname{atan} 2\left(y_{\mathrm{WP}}-y_{e}, x_{\mathrm{WP}}-x_{e}\right) \begin{cases}+\frac{\pi}{2} & \text { if SPEC2 }=\mathrm{CW} \\ -\frac{\pi}{2} & \text { if SPEC2 }=\mathrm{CCW}\end{cases}
$$

where $x_{\mathrm{WP}}$ and $y_{\mathrm{WP}}$ are the coordinates of WP.

\section{C.2.2 Vertical profile}

Level

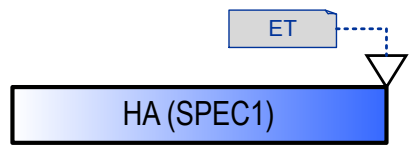

Figure C.14: 'Level' template.

This template represents a level flight where the quadrotor holds its altitude, as shown in figure C.14. A composite created from this template is denoted 'Level $\langle$ SPEC1,ET〉', where the undefined parameters are: 
1. SPEC1: a set of AIDL specifiers indicating the possible reference frames (EARTH or AIR).

2. ET: the end trigger of the composite.

\section{Ascent/Descent}

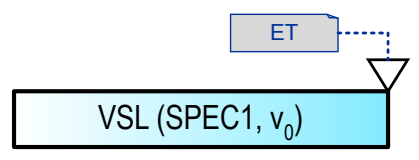

Figure C.15: 'Ascent/Descent' template.

This template represents an ascent or descent manoeuvre, as shown in figure C.15. A composite created from this template is denoted 'Ascent/Descent $\left.\left\langle\mathrm{SPEC} 1, v_{0}, \mathrm{ET}\right\rangle\right\rangle^{\prime}$, where the undefined parameters are:

1. SPEC1: a set of AIDL specifiers indicating the possible reference frames (EARTH or AIR).

2. $v_{0}$ : an interval of possible vertical speeds (either $z_{e}$ or $z_{a}$, depending on the reference frame).

3. ET: the end trigger of the composite.

\section{C.2.3 Lateral and vertical profiles}

\section{Direct-to point}

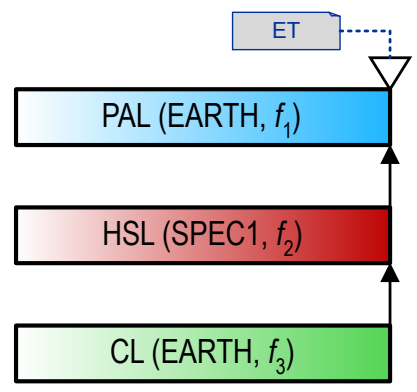

Figure C.16: 'Direct-to point' template.

This template represents a flight in straight line towards a three-dimensional (3D) point, as shown in figure C.16. A composite created from this template is denoted 'Direct-to point $\left\langle\mathrm{SPEC} 1, v_{0}, h_{0}, \mathrm{ET}\right\rangle$ ', where the undefined parameters are:

1. SPEC1: a set of AIDL specifiers indicating the possible reference frames (EARTH or AIR). 
2. $v_{0}$ : an interval of possible vertical speeds (either $\dot{z}_{e}$ or $\dot{z}_{a}$, depending on the reference frame).

3. $h_{0}$ : an interval of possible target altitudes $\left(z_{e}\right)$.

4. ET: the end trigger of the composite, which must be a waypoint trigger.

The function $f_{1}$ defines the geometric path angle $\gamma_{e}$ as:

$$
f_{1}=\arcsin \frac{h_{0}-z_{e}}{\sqrt{\left(x_{\mathrm{WP}}-x_{e}\right)^{2}+\left(y_{\mathrm{WP}}-y_{e}\right)^{2}+\left(h_{0}-z_{e}\right)^{2}}}
$$

where $x_{\mathrm{WP}}$ and $y_{\mathrm{WP}}$ are the coordinates of the waypoint in ET.

If using an Earth-fixed reference frame (EARTH specifier), the function $f_{2}$ defines the ground speed $v_{g}, f_{2}=v_{0} \cos \gamma_{e}$. Otherwise, if using a body-fixed Earth-normal reference frame (AIR specifier), the function $f_{2}$ defines the horizontal speed $v_{h}, f_{2}=v_{0} \cos \gamma_{a}$.

The function $f_{3}$ defines the geometric course $\chi_{e}$ as:

$$
f_{3}=\operatorname{atan} 2\left(y_{\mathrm{WP}}-y_{e}, x_{\mathrm{WP}}-x_{e}\right)
$$

\section{C.2.4 Orientation profile}

\section{Face-to point}

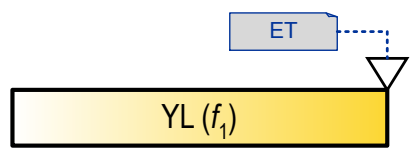

Figure C.17: 'Face-to point' template.

This template represents a manoeuvre where the quadrotor is constantly facing the same point, as shown in figure C.17. A composite created from this template is denoted 'Face-to point $\langle\mathrm{WP}, \mathrm{ET}\rangle$ ', where the undefined parameters are:

1. WP: the $2 \mathrm{D}$ waypoint towards which the quadrotor must be oriented.

2. ET: the end trigger of the composite.

The function $f_{1}$ defines the yaw angle $\psi$ as:

$$
f_{1}=\operatorname{atan} 2\left(y_{\mathrm{WP}}-y_{e}, x_{\mathrm{WP}}-x_{e}\right)
$$

where $x_{\mathrm{WP}}$ and $y_{\mathrm{WP}}$ are the coordinates of WP. 


\section{Face forward}

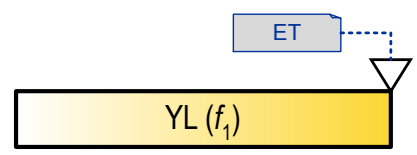

Figure C.18: 'Face forward' template.

This template represents a manoeuvre where the quadrotor is constantly facing the direction of movement, as shown in figure C.18. A composite created from this template is denoted 'Face forward $\langle\mathrm{SPEC} 1, \mathrm{ET}\rangle$ ', where the undefined parameters are:

1. SPEC1: a set of AIDL specifiers indicating the possible reference frames (EARTH or AIR).

2. ET: the end trigger of the composite.

The function $f_{1}$ defines the yaw angle $\psi$ to be equal to the geometric course $\left(f_{1}=\chi_{e}\right)$ or the aerodynamic course $\left(f_{1}=\chi_{a}\right)$, depending on the reference frame specified.

\section{Hold orientation}

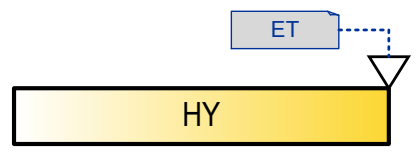

Figure C.19: 'Hold orientation' template.

This template represents a manoeuvre where the quadrotor is kept constant, as shown in figure C.19. A composite created from this template is denoted 'Hold orientation $\langle\mathrm{ET}\rangle$ ', where the undefined parameter is the end trigger of the composite, ET. 



\section{Appendix D}

\section{Formal specification of intent-based languages}

This appendix defines the text notation used in part II for the description of the language hierarchy. Most of this notation has already been outlined in the corresponding chapters, but some formal details may have been omitted. For completion, a formal description of this notation - i.e., languages' syntax - is provided in this appendix.

The syntax of these languages can be described using a context-free grammar [14]. A grammar consists of a number of nonterminal symbols (also called variables), an alphabet $\Sigma$ containing all the terminal symbols, and a series of production rules. These rules define how the words in the language are generated, and they are of the form:

$$
a \rightarrow \alpha
$$

where $a$ is a nonterminal symbol and $\alpha$ is a string of zero or more terminal and nonterminal symbols. In this appendix, nonterminal symbols use text in italics, whereas terminal symbols use bold text and symbols. Some production rules may produce no symbols, that is, they produce an empty string. The symbol $\lambda$ is used to denote this empty string. One of the nonterminal symbols in the language is designated as the start variable. Therefore, all the words in the language can be produced by recursively expanding the start variable using the production rules in the grammar until no nonterminal symbols remain.

The notation used for production rules can be extended with additional forms that make a grammar definition more compact. In the grammars described in this appendix, the following three extensions are used:

- A compact form can be used to represent several production rules that expand the same nonterminal symbol. This form, which can be written as

$$
a \rightarrow \alpha|\beta| \ldots \mid \zeta
$$


is equivalent to

$$
\begin{gathered}
a \rightarrow \alpha \\
a \rightarrow \beta \\
\vdots \\
a \rightarrow \zeta
\end{gathered}
$$

- Additionally, the form $\{\alpha\}$ represents a nonterminal symbol that can be expanded to produce the string $\alpha$ zero or more times. Therefore, the production rule

$$
a \rightarrow \alpha\{\beta\} \gamma
$$

is equivalent to

$$
\begin{aligned}
& a \rightarrow \alpha b \gamma \\
& b \rightarrow \beta b \mid \lambda
\end{aligned}
$$

- Finally, the form $(\mathbf{a}-\mathbf{z})$ represents a nonterminal symbol that can be expanded to produce any of the terminal symbols from a to $\mathbf{z}$. The same form can be used for any other interval of symbols, such as $(\mathbf{A}-\mathbf{Z})$ or (0-9). Thus, the following rule

$$
a \rightarrow \alpha(\mathbf{A}-\mathbf{Z}) \beta
$$

is equivalent to

$$
\begin{aligned}
& a \rightarrow \alpha b \beta \\
& b \rightarrow \mathbf{A}|\mathbf{B}| \ldots \mid \mathbf{Z}
\end{aligned}
$$

For example, a grammar that generates all the natural numbers can be defined using the extended notation above. This grammar has a start variable nat and can be written as:

$$
n a t \rightarrow \mathbf{0} \mid(\mathbf{1}-\mathbf{9})\{(\mathbf{0}-\mathbf{9})\}
$$

The following sections present the particular grammars corresponding to each of the languages in the hierarchy described in part II.

\section{D.1 Quadrotor AIDL}

In this thesis, the textual notation of the quadrotor AIDL (QR-AIDL) has only been used to describe the contents of the instructions. For completion, this section presents how to build a complete QR-AIDL word using the textual notation. Basically, an aircraft intent expressed in QR-AIDL consists of four threads separated by the plus $(+)$ sign. Each thread contains one or more instructions, separated by a comma $($,$) .$ 
Any aircraft intent expressed in QR-AIDL can be expanded from the start variable $a i$.

$$
\text { ai } \rightarrow \text { thread }_{1}+\text { thread }_{2}+\text { thread }_{3}+\text { thread }_{4}
$$

Every thread must contain at least one instruction of the corresponding type.

$$
\begin{aligned}
\text { thread }_{1} & \rightarrow \text { inst }_{1}\left\{, \text { inst }_{1}\right\} \\
\vdots & \\
\text { thread }_{4} & \rightarrow \text { inst }_{4}\left\{, \text { inst }_{4}\right\}
\end{aligned}
$$

Instructions are produced by the nonterminal symbols inst $_{i}(i \in\{1,2,3,4\})$. The reader should be more familiar with the syntax used for instructions, as examples have been provided throughout this thesis. They consist of the name of the effect of the instruction followed by a list of parameters in parenthesis:

$$
\begin{aligned}
\text { inst }_{1} & \rightarrow \text { eff } \\
1 & (\{\text { param, }\} \text { trig }) \text { opttag } \\
& \vdots \\
\text { inst }_{4} & \rightarrow \text { eff } \\
\text { opttag } & \rightarrow \text { tag } \mid \lambda \\
\text { tag } & \rightarrow \text { \#name }
\end{aligned}
$$

Instructions may have an optional tag. A tag can be used to give a name to a particular instruction so it can be referenced from within another instruction (e.g., in linked and auto triggers, as it is later shown). A name is an alphanumeric string that contains any combination of letters, numerical digits, and some symbols (dashes and underscores).

$$
\begin{aligned}
\text { name } & \rightarrow \text { letterordigit }\{\text { letterordigit }\} \\
\text { letterordigit } & \rightarrow(\mathbf{0}-\mathbf{9})|-|-|(\mathbf{a}-\mathbf{z})|(\mathbf{A}-\mathbf{Z})
\end{aligned}
$$

The effects available in each thread are determined by the nonterminal symbols effi $(i \in\{1,2,3,4\})$.

$$
\begin{aligned}
& \text { eff }_{1} \rightarrow \text { TL | HT | OLT | AL | HA | VSL | HVS } \\
& \text { eff }_{2} \rightarrow \text { RL | HR | OLR | AL | PL | HP | OLP | CL | HC | HSL | HHS } \\
& \text { eff }_{3} \rightarrow \text { RL | HR | OLR | AL | PL | HP | OLP | CL | HC | HSL | HHS } \\
& \text { eff }_{4} \rightarrow \text { YL } \mid \text { HY } \mid \text { OLY }
\end{aligned}
$$

Parameters, as discussed in chapter 6, may be function parameters, magnitude parameters, or specifier parameters. In the QR-AIDL there are only two specifiers, EARTH and AIR.

$$
\begin{aligned}
\text { param } & \rightarrow \text { funcparam } \mid \text { magparam } \mid \text { specparam } \\
\text { funcparam } & \rightarrow \text { expr } \\
\text { magparam } & \rightarrow \text { mag } \\
\text { specparam } & \rightarrow \text { EARTH } \mid \text { AIR }
\end{aligned}
$$


The nonterminal symbol expr represents mathematical expressions, which may use all common mathematical operations and functions. The detailed grammar for mathematical expressions is not covered in this appendix but can be found elsewhere $[14,159]$. Also, the nonterminal symbol mag represents magnitudes, which consist of a real number (realn) with possibly a unit name.

$$
\begin{aligned}
\text { mag } & \rightarrow \text { realn optunit } \\
\text { realn } & \rightarrow \text { nat optdec } \\
\text { nat } & \rightarrow \mathbf{0} \mid(\mathbf{1}-\mathbf{9})\{(\mathbf{0}-\mathbf{9})\} \\
\text { optdec } & \rightarrow \cdot\{(\mathbf{0}-\mathbf{9})\} \mid \lambda \\
\text { optunit } & \rightarrow \text { unit } \mid \lambda \\
\text { unit } & \rightarrow \mathbf{f t}|\mathbf{N M}| \mathbf{d e g}|\mathbf{k t}| \ldots
\end{aligned}
$$

Triggers may be of any of the types described in chapter 6. Fixed, floating, linked, or auto triggers can be used in the QR-AIDL.

$$
\begin{aligned}
\text { trig } & \rightarrow \text { fixedtrig } \mid \text { floattrig } \mid \text { linkedtrig } \mid \text { autotrig } \\
\text { fixedtrig } & \rightarrow t=\text { mag } \\
\text { floattrig } & \rightarrow \text { expr }=\exp r \mid \text { expr } \geq \operatorname{expr} \mid \text { expr } \leq \operatorname{expr} \\
\text { linkedtrig } & \rightarrow \text { tag } \\
\text { autotrig } & \rightarrow \text { expr }=\exp r @ \text { tag }
\end{aligned}
$$

Fixed triggers can only refer to a fixed point in time. Floating triggers contain a condition represented as an equation or inequation. Linked triggers refer to the end trigger of another instruction using a tag. Auto triggers set a condition (expressed as an equation) on a future time defined by the end trigger of an instruction referred using a tag.

\section{D.2 Intent composite description language}

A formalized description of the textual notation for the intent composite description language (ICDL), which was introduced in chapter 7 , is provided here. As this language is based on the AIDL, some terminal and nonterminal symbols defined in the previous section are used here.

Any composite expressed in ICDL can be generated from the start variable comp:

$$
\begin{aligned}
& \text { comp } \rightarrow \text { elcomp } \\
& \text { comp } \rightarrow(\text { comp }) \\
& \text { comp } \rightarrow \text { comp } \oplus \text { comp } \\
& \text { comp } \rightarrow \text { comp } \otimes \text { comp } \\
& \text { comp } \rightarrow(\text { comp })[\text { cnd optiter }] \\
& \text { comp } \rightarrow(\text { comp } \mid \text { comp })[\text { cnd }]
\end{aligned}
$$


Note that the grammar provided in this appendix is designed only to generate correct ICDL words, but it does not take into account the precedence of operators. Thus, the grammar presented here is ambiguous. That is, a composite $C_{1} \otimes C_{2} \oplus C_{3}$ could be interpreted as $\left(C_{1} \otimes C_{2}\right) \oplus C_{3}$ and as $C_{1} \otimes\left(C_{2} \oplus C_{3}\right)$. While the former interpretation would be the correct one (as the transversal composition has precedence over longitudinal composition), both produce syntactically correct ICDL words.

The conditional branching and conditional loop require a condition to be defined. Additionally, the conditional loop may set a minimum or maximum number of iterations. The syntax of these elements is defined by the following production rules.

$$
\begin{aligned}
c n d & \rightarrow \text { expr }=\exp r \mid \text { expr } \geq \exp r \mid \text { expr } \leq \operatorname{expr} \\
\text { optiter } & \rightarrow \text { : nat: nat }
\end{aligned}
$$

Elementary composites (elcomp) are generated using the following production rule:

$$
\begin{aligned}
\text { elcomp } & \rightarrow \text { templname }\langle\text { cparams }\rangle \text { opttag } \\
\text { templname } & \rightarrow \text { name } \mid \lambda
\end{aligned}
$$

where templname is the name of the template used, and cparams is the list of composite parameters. Composite parameters are much like the parameters in the AIDL, but magnitude parameters become interval parameters, and specifier parameters become set parameters. Also, parameter lists are allowed.

$$
\begin{aligned}
\text { cparams } & \rightarrow \text { cparam }\{, \text { cparam }\} \\
\text { cparam } & \rightarrow \text { intparam } \mid \text { setparam } \mid \text { paramlist } \mid \text { ctrig } \\
\text { intparam } & \rightarrow \text { realn..realn }: \text { mag } \mid \text { mag } \\
\text { setparam } & \rightarrow\{\text { specparam }\{, \text { specparam }\}\} \\
\text { paramlist } & \rightarrow\{\} \mid\{\text { intparam }\{, \text { intparam }\}\} \mid\{\text { setparam }\{, \text { setparam }\}\}
\end{aligned}
$$

The end triggers in a composite also can take advantage of interval parameters. Additionally, a new type of trigger, the waypoint trigger, is available in this language.

$$
\begin{aligned}
\text { ctrig } & \rightarrow \text { cfixedtrig } \mid \text { cfloattrig } \mid \text { linkedtrig } \mid \text { cautotrig } \mid \text { wpttrig } \\
\text { cfixedtrig } & \rightarrow t=\text { intparam } \\
\text { cfloattrig } & \rightarrow \text { floattrig } \mid \text { expr }=\text { intparam } \mid \text { expr } \geq \text { intparam } \mid \text { expr } \leq \text { intparam } \\
\text { cautotrig } & \rightarrow \text { autotrig } \mid \text { expr }=\text { intparam @ tag } \\
\text { wpttrig } & \rightarrow \text { wpt }
\end{aligned}
$$

Only linked triggers (linkedtrig) remain with the exact same syntax as in the ICDL. Fixed triggers (fixedtrig), floating triggers (floattrig), and auto triggers (autotrig) allow having a magnitude interval at one side of the equation or inequation. However, every valid AIDL trigger is a valid ICDL trigger. Waypoint triggers are specified using a waypoint. 
Waypoints may be described by the waypoint's name and an optional tolerance distance; or as a sequence of longitude, latitude, and tolerance distance.

$$
\begin{aligned}
\text { wpt } & \rightarrow \text { name opttol } \mid[\text { mag, mag, intparam }] \\
\text { opttol } & \rightarrow[\text { intparam }] \mid \lambda
\end{aligned}
$$

\section{D.3 Flight intent description language}

The grammar that describes the flight intent description language (FIDL) is presented in this section. As this language is based on the previously defined languages, some of the terminal and nonterminal symbols used in previous sections are used in this section. fi.

A flight intent expressed in the FIDL can be produced from the start variable

$$
\begin{aligned}
f i & \rightarrow \text { FLIGHT_SEGMENTS (aggregation) optconst optobj } \\
\text { optconst } & \rightarrow \text { CONSTRAINTS }(\text { constraint-set) } \mid \lambda \\
\text { optobj } & \rightarrow \text { OBJECTIVES (objective-set) } \mid \lambda
\end{aligned}
$$

The syntactical rules for the aggregation of flight segments are similar to those of composites operations. Note that a flight segment is completely defined using a composite, so these rules can be written as:

$$
\begin{aligned}
& \text { aggregation } \rightarrow \text { comp } \\
& \text { aggregation } \rightarrow \text { aggregation } ; \text { aggregation } \\
& \text { aggregation } \rightarrow\{\text { aggregation } \mid \text { aggregation }\}[\text { cnd }] \\
& \text { aggregation } \rightarrow\{\text { aggregation }\}[\text { cnd optiter }]
\end{aligned}
$$

The constraint set is defined using a sequence of constraints separated by a semicolon. Each constraint, as defined in chapter 8, contains a domain of application (DoA), an effect, and a priority.

$$
\begin{aligned}
\text { constraint-set } & \rightarrow \text { constraint }\{; \text { constraint }\} \\
\text { constraint } & \rightarrow \text { optpriority conseff@ optweak doa } \\
\text { optpriority } & \rightarrow(\text { realn }) \mid \lambda \\
\text { optweak } & \rightarrow{ }^{(\mathrm{w})} \mid \lambda
\end{aligned}
$$

The effect can be defined using a mathematical inequation or using the name of a path (e.g., STAR, SID, airway).

$$
\begin{aligned}
& \text { conseff } \rightarrow \text { effmath } \mid \text { effroute } \\
& \text { effmath } \rightarrow \text { expr } \leq \text { expr } \leq \text { expr } \mid \text { expr } \leq \text { expr } \\
& \text { effroute } \rightarrow \text { name }
\end{aligned}
$$


The DoA can be defined using a mathematical inequation or a geometric space (i.e., a point, a segment, or a volume). Also, the universal DoA, denoted $\Omega$, can be used.

$$
\begin{aligned}
\text { doa } & \rightarrow \text { doamath } \mid \text { doapoint } \mid \text { doasegmt } \mid \text { doavol } \mid \mathbf{\Omega} \\
\text { doamath } & \rightarrow \text { expr } \leq \text { expr } \mid \text { expr } \geq \text { expr } \\
\text { doapoint } & \rightarrow \text { wpt optaltitude } \\
\text { doasegmt } & \rightarrow\{\text { wpt, wpt }\} \text { optaltitude } \\
\text { doavol } & \rightarrow\{\text { wpt, wpt, wpt }\{, \text { wpt }\}\} \text { optaltitude } \\
\text { optaltitude } & \rightarrow[\text { mag, mag }] \mid \lambda
\end{aligned}
$$

The objective set is denoted as a sequence of objectives separated by a semicolon. Objectives have a weight, an effect, and a DoA. The weight is a real number, the effect is a mathematical expression, and DoAs have the same purpose and syntax as in constraints.

$$
\begin{aligned}
\text { objective-set } & \rightarrow \text { objective }\{; \text { objective }\} \\
\text { objective } & \rightarrow \text { weight objeff@ doa } \\
\text { weight } & \rightarrow(\text { realn }) \\
\text { objeff } & \rightarrow \text { expr }
\end{aligned}
$$

\section{D.4 Mission intent description language}

This section includes a grammar that describes the syntax of the mission intent description language (MIDL). Again, as this language is built on top of the FIDL, some symbols defined in previous sections are used in this section.

Any mission intent expressed in MIDL can be generated from the start variable $m i$.

$$
\begin{aligned}
m i & \rightarrow \text { TASKS }(\text { task-list }) \text { optmconst optmobj } \\
\text { optmconst } & \rightarrow \text { CONSTRAINTS }(\text { mission-const-set }) \mid \lambda \\
\text { optmobj } & \rightarrow \text { OBJECTIVES }(\text { mission-obj-set }) \mid \lambda
\end{aligned}
$$

The task list consist of a sequence of tasks separated by semicolons. As described in chatper 9, each task has an effect, a specification of the candidate aircraft, the precondition and the minimum and maximum number of times that the task must be executed. All tasks also have an identifier (a tag) that gives it a name so it can be later referenced.

$$
\begin{aligned}
\text { task-list } & \rightarrow \text { task }\{; \text { task }\} \\
\text { task } & \rightarrow\{\text { taskeff; candidates; precnd; nat; nat }\} \text { tag }
\end{aligned}
$$

The effect of a task (taskeff) may be specified using the FIDL or using a template. The parameters that can be used in a MIDL template are analogous 
to those of the ICDL.

$$
\begin{aligned}
& \text { taskeff } \rightarrow f i \\
& \text { taskeff } \rightarrow \text { name }\langle\text { cparams }\rangle
\end{aligned}
$$

The candidate aircraft (candidates) may be specified by an aircraft identifier (a name) or a combination of requisites on the aircraft's payload, type, or state.

$$
\begin{aligned}
\text { candidates } & \rightarrow \text { name } \mid \text { any } \mid \text { acftreq } \\
\text { acftreq } & \rightarrow \text { acftreq } \wedge \text { acftreq } \mid \text { acftreq } \vee \text { acftreq } \mid \neg \text { acftreq } \\
\text { acftreq } & \rightarrow \text { equips }(\text { payload }) \mid \text { type }(\text { acfttype }) \mid \text { cnd } \\
\text { payload } & \rightarrow \text { name } \\
\text { acfttype } & \rightarrow \text { quadrotor } \mid \text { fixed-wing }
\end{aligned}
$$

The precondition (precnd) may also include a combination of mathematical conditions using logic operations. In this case, the status function is available in the mathematical expressions.

$$
\begin{aligned}
& \text { precnd } \rightarrow \text { precnd } \wedge \text { precnd } \mid \text { precnd } \vee \text { precnd } \mid \neg \text { precnd } \\
& \text { precnd } \rightarrow \text { cnd }
\end{aligned}
$$

The constraint set (mission-const-set) consists on a sequence of constraints separated by semicolons. Each constraint may be a global constraint (which have the same syntax as FIDL constraints) or a mission constraint. Mission constraints have any number of declarations, an effect, and a DoA.

$$
\begin{aligned}
\text { mission-const-set } & \rightarrow \text { mission-const }\{\text {; mission-const }\} \\
\text { mission-const } & \rightarrow \text { constraint } \\
\text { mission-const } & \rightarrow\{\text { decl, }\} \text { mconseff@mdoa } \\
\text { decl } & \rightarrow \text { var }=\text { candidates } \\
\text { var } & \rightarrow \text { name } \\
\text { mconseff } & \rightarrow \text { conseff } \mid \forall \text { quantspec conseff } \mid \exists \text { quantspec conseff } \\
\text { quantspec } & \rightarrow \# \mid \text { var }\{, \text { var }\} \\
\text { mdoa } & \rightarrow \text { doa } \mid \text { var } \neq \text { var } \mid \text { var=var }
\end{aligned}
$$

Finally, the objective set (mission-obj-set) is built as a sequence of objectives separated by a seimicolon. The syntax of a mission objective is very similar to that of the mission constraints.

$$
\begin{aligned}
\text { mission-obj-set } & \rightarrow \text { mission-obj }\{; \text { mission-obj }\} \\
\text { mission-obj } & \rightarrow\{\text { decl, }\} \text { mobjeff@mdoa } \\
\text { mobjeff } & \rightarrow \text { expr }
\end{aligned}
$$

